\title{
INORGANIC NANOARCHITECTURES BY ORGANIC SELF-ASSEMBLY
}

A dissertation submitted for the degree of Doctor of Philosophy by

Stefan Guldin

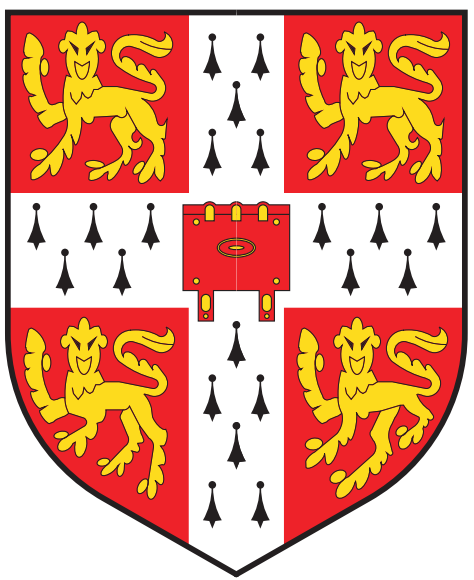

University of Cambridge Cavendish Laboratory

Jesus College

February 2012 

Foreword by Prof. Ullrich Steiner 



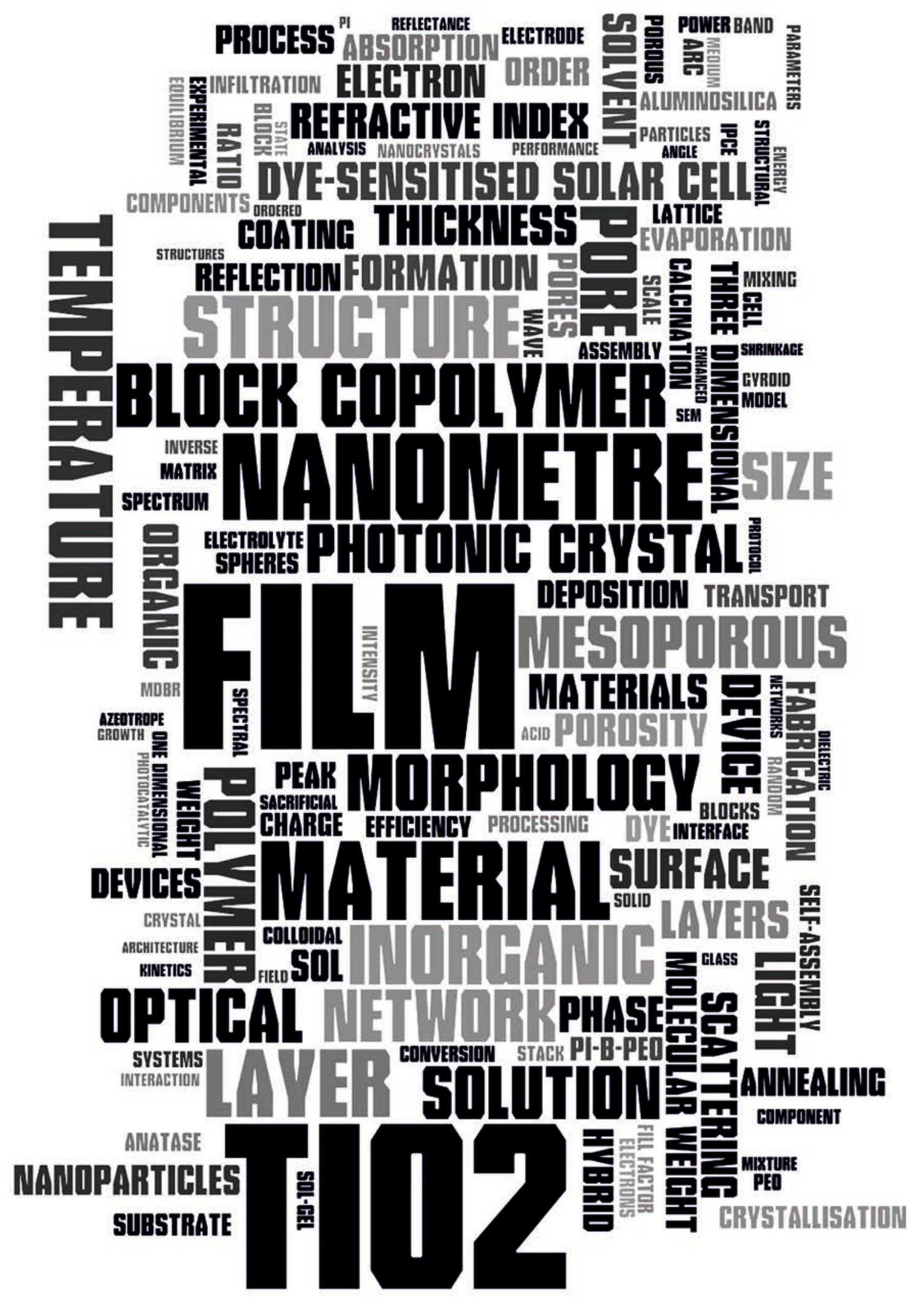

The dissertation in a nutshell. 



\section{Preface}

The focus of this thesis lies in the use of soft matter self-assembly as a design tool for the bottom-up manufacture of functional inorganic nanoarchitectures. Several routes are explored, starting from structure formation of hybrid materials on the 10-nm length scale to their application in photonic and optoelectronic devices. Considering the underlying principles and accomplished results, the outline of the thesis is structured as follows:

Chapter 1 - 4: Introduction to fundamental aspects of experimental and techniques.

Chapter 5-7: Material morphology control and implementations in thin-film architectures.

Chapter 8 - 10: Photonic structures designed by soft-matter self-assembly.

The fundamental principles of soft-matter self-assembly are discussed in Chapter 1, including the driving mechanisms for structure formation in block copolymers (BCP), colloidal and hybrid systems as well as their implementation in soft nanotechnology. The optics of thin films and interfaces is subject of Chapter 2, an important aspect of many results presented in the following chapters. Dye-sensitised solar cells were a main device platform for the implementation and validation of the proposed material functionality. Chapter 3 is therefore devoted to explaining the main principles of this photovoltaic concept and describing current challenges for further efficiency improvements. The final chapter of the introductory part, Chapter 4 , then represents an overview of the material fabrication and characterisation techniques that were employed in the course of this work.

Experimental results of the doctoral research are presented in Chapters 5 to 10. In Chapter 5 the developed routes for BCP-induced structure control of inorganic nanomaterials are described. These findings were of central importance for many applications presented in the following. The morphological control that is offered by soft matter co-assembly allows to finely control crystallisation and electronic properties of mesoporous networks. This is explored in Chapter 6, which contains the study of crystal growth in $\mathrm{BCP}$-derived $\mathrm{TiO}_{2}$ nanostructures during high temperature calcination and its effect on the performance of dye-sensitised solar cells. The sacrificial use of organic material suffers from considerable shrinkage, occurring during the several fabrication steps from solution processing of the hybrid mixture to the crystallisation of the inorganic material at high temperatures. In Chapter 7, a protocol is developed that enables to overcome this drawback and allow the formation of sufficiently thick films without the typically occurring crack formation and delamination. 
Soft matter self-assembly allows fine tuning of inorganic material formation on the $10-$ $500 \mathrm{~nm}$ length scale and therefore enables to design material architectures with interesting photonic properties. This is the motivation for Chapters $7-10$. Control over sub-wavelength properties, such as pore dimensions, pore volume and film thickness of the resulting inorganic films enabled the stacking of individual mesoporous layers into a multilayer lattice of alternating high and low refractive index, a mesoporous Bragg reflector (MDBR). A BCP-based route to tunable MDBRs is presented in Chapter 8. The combination of $\mathrm{BCP}$ and colloidal self-assembly was used to establish a $\mathrm{TiO}_{2}$ electrode architecture, where a high surface area mesoporous underlayer is coupled to an optically and electronically active three dimensional photonic crystal. This concept is demonstrated in Chapter 9, including the experimental characterisation of such a photonic electrode architecture in terms of light harvesting and device performance in a dye-sensitised solar cell. Detailed control over the pore architecture allowed the fabrication of dielectric thin films with such ultralow refractive indices that they enable the incorporation of high refractive index photocatalytic $\mathrm{TiO}_{2}$ nanocrystals. A new materials strategy for self-cleaning antireflective coatings is presented in Chapter 10, which is compatible with low processing temperatures and therefore applicable for a variety of substrate materials.

Finally, Chapter 11 concludes the work by summarising the results and identifying possible directions for future studies. 


\section{Acknowledgements}

A great number of people helped, taught, inspired and cheered me during my doctorate, which made my time in Cambridge only so incredibly enjoyable and successful.

I am very grateful to my supervisor Prof. Ullrich Steiner, who provided me with such great working opportunities and introduced me to interesting projects and people. Like a true mentor, he guided me through the world of science, always supportive, encouraging, knowledgable and full of ideas. Many of my skills and abilities today are closely linked to his teaching and leadership. Thank you Ulli, you shine bright as a role model for future scientists.

I was lucky to be member of the Thin Film and Interfaces group, an absolutely great homebase for scientific research. Thanks to all current and past members, who made me feel welcome and appreciated at the Cavendish, including Alessandro Sepe, Alexandre Nicolas, David Barbero, Ellie Kim, Frederic Winzenrieth, Gen Kamita, Kai Scherer, Katherine Thomas, Li Li, Nataliya Yufa, Pedro Cunha, Pola Goldberg-Oppenheimer, Sarah Rong, Stefano Salvatore, Xiaoyuan Sheng and Yuan Zhou. Alexander Finnemore was always approachable for my thoughts and questions and truly cheered up my days in the lab - thanks dude. The kindhearted support of Mathias Kolle boosted many scientific findings alongside with his beautiful illustrations. Maik Scherer was of great help for many things, in particular when writing and fighting with $\mathrm{LT}_{\mathrm{E}} \mathrm{X}$. A number of post-docs were absolutely crucial for the success of my work: Grazie mille to Silvia Vignolini, who gave me generous support concerning all optical aspects of this work. Sven Hüttner set me on the track for this adventure and was a constant source of help ever since. Peter Kohn was a true source of wisdom and an everlasting discussion partner. Thanks a lot, Team TFI.

The Kapitza Building is real hot spot for good science and I want to acknowledge the great help and stimulation I received from the Nanophotonics and Optoelectronics groups. Thanks to Prof. Jeremy Baumberg for generously letting me use the Nanophotonics facilities and advice whenever needed. Niraj Lal was a true buddy inside and outside the optics lab. My desk neighbor Dinesh Kabra was a constant source of happiness and knowledge. The same applies to Sumeet Mahajan, thank you all for your time and helpfulness and the interesting discussions. A number of people helped me improving this dissertation by proofreading and valuable discussions. My gratitude goes to Nicky Humphry-Baker, Alex Finnemore, James Hugall, Kevin Musselman, Matthew Hawkeye and Niraj Lal, your efforts are very much appreciated.

The outcome of this thesis would not have been possible without the excellent collabo- 
rations with our partners at Cornell University and Oxford. A big thank you goes to Prof. Ulrich Wiesner for his generous support and hospitality during my three-month research stay at Cornell. My time in Ithaca will always be of great memories and all members of his groups were extremely helpful and welcoming. Morgan Stefik was absolutely crucial for the success of my work. He synthesised many of the polymers and let me share his wisdom in countless discussions - thank you very much. The Snaith group in Oxford was equally important and stimulating. Dr. Henry Snaith was a constant fountain of enthusiasm and ideas. Together with Pablo Docampo and Priti Tiwana, who fostered my $\mathrm{TiO}_{2}$ electrodes to reach new records, the were a real trio de force. Pablo and me spent a lot of time together on the phone, computer and in the lab and I am happy that this led to some excellent results, muchas gracias compañero. Much appreciated collaborators were also Dr. Caterina Ducati, Giorgio Divitini and Prof. Dominik Eder, thanks for your skillful materials characterisation as well as help and advice.

The support staff in the Cavendish was always very helpful and provided a well functioning working environment. I want to express my gratitude and appreciation to Jean Phillips, Stefani Gerber, Tracy Inman, Colin Edwards, Nigel Palfrey, Owen Dunn, Pete Bone, Richard Gymer and Suresh Mistry. My examiners Prof. Thomas Bein and Prof. Richard Friend challenged me in the defence of this thesis and gave me feedback, confidence and appreciation in my doctoral work - thank you very much for this privilege. I want to acknowledge Jesus College and its members for providing me with a great scholarly atmosphere, including such a nice accommodation and an active College life. All of this would not have been possible without the necessary financial resources and I want to express my gratitude for the generous support by the KAUST research initiative on energy and sustainability (No. KUS-C1-018-02) as well as the NanoDTC Associate programme of the Nano Science \& Technology Doctoral Training Centre Cambridge.

My family, Marie-Luise, Wilhelm and Thomas Guldin have been a constant source of trust and happiness and fostered the values I belief in today. Thank you for your confidence and love.

Supervisors, coworkers, friends and family - all played a crucial role and I owe them my deepest gratitude. But there is only one person who lets my star shine, my wonderful wife Lisa Pfaffenrath. Thank you so much for moving with me to the land of fog and rain to find out how exciting and sunny it actually is. Thanks for exploring with me the miracles of life and supporting me in whatever situation. Our time here in Cambridge was absolutely fabulous and I am glad that we will explore any future challenges together in a team. 


\section{List of abbreviations}

1D

2D

3D

ARC

BCC

$\mathrm{BCP}$

BET

BJH

DLS

DSC

$\mathrm{e}^{-1}$

EM

FWHM

FTO

FCC

IPCE

MDBR

NP

PC

PEO- $b$-PPO- $b$-PEO

PET

PHB- $b$-PEO

PI- $b$-PEO

SAXS

SC

SDA

SEM

TCE

TEM

TGA

WAXS one-dimensional

two-dimensional

three-dimensional

antireflective coatings

body-centred cubic

block copolymer

Brunauer, Emmett \&Teller

Barrett, Joyner \& Halenda

dynamic light scattering

dye-sensitised solar cell

electron

electromagnetic

full width at half maximum

fluorine-doped tin oxide

face-centered cubic

incident photon to electron conversion efficiency

mesoporous Bragg reflector

nanoparticle

photonic crystal

poly(ethylene oxide-block-propylene oxide-block-ethylene oxide) - Pluronic poly(ethylene terephthalat)

poly(ethylene-co-butylene-block-ethylene oxide) - KLE

poly(isoprene-block-ethylene oxide)

small angle $\mathrm{X}$-ray scattering

simple cubic

structure directing agent

scanning electron microscopy

transparent conducting electrode

transmission electron microscopy

thermogravimetric analysis

wide-angle X-ray scattering 


\section{Contents}

1 Self-assembly of soft matter $\quad 1$

1.1 Self-assembly in nature . . . . . . . . . . . . . . . . . . . . . . . 2

1.2 Self-assembly in synthetic systems . . . . . . . . . . . . . . . . . . . 3

1.2.1 Phase separation of polymer blends . . . . . . . . . . . . . . 3

1.2.2 Structure formation in block copolymer systems . . . . . . . . . . . . 5

1.2.3 Block copolymer-directed co-assembly of inorganic material . . . . . . . . 8

1.2.4 Colloidal self-assembly .................... 11

Bibliography ........................... 15

2 Optical aspects of thin films and interfaces $\quad 21$

2.1 Propagation of light at an optical interface - reflection and refraction . . . . . . . . . 21

2.2 Thin film interference . . . . . . . . . . . . . . . . . . . . 23

2.3 Model of multilayer reflectivity . . . . . . . . . . . . . . . . . . . . . . 24

2.3.1 Rouard's method ...................... . . 24

2.3.2 Transfer matrix method ................... 25

2.4 From periodic multilayer stacks to photonic crystals . . . . . . . . . . . . . . . . 28

2.5 Optical properties of porous thin films . . . . . . . . . . . . . . . . . . 29

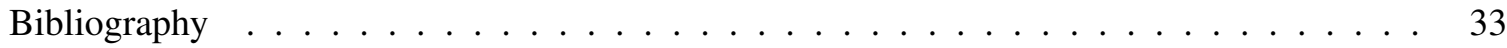

3 Structure-function interplay in dye-sensitised solar cells 37

3.1 Novel electrode structures for enhanced charge carrier transport . . . . . . . . . . . 43

3.2 Photonic device architectures for enhanced light absorption . . . . . . . . . . . . . . 48

Bibliography ......................... 51

4 Experimental and analytical techniques $\quad 59$

4.1 Materials fabrication ........................ 59

4.1.1 Poly(isoprene-block-ethylene oxide) copolymers . . . . . . . . . . . . 59

4.1.2 Sol-gel chemistry ...................... 60

4.1.3 Thin film deposition ..................... 62

4.2 Materials characterisation . . . . . . . . . . . . . . . . . . . 64

4.2.1 Imaging techniques . . . . . . . . . . . . . . . . . . . 64

4.2.2 Scattering techniques .................... 67

4.2.3 Spectroscopy techniques ................... 72

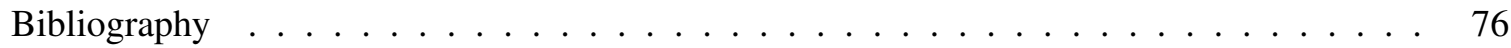

5 Block copolymer-induced structure control for inorganic nanomaterials 81

5.1 Introduction . . . . . . . . . . . . . . . . . . . . . 81

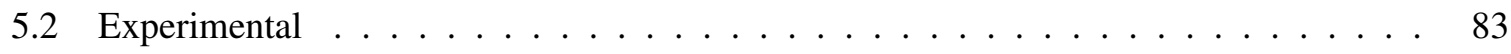


5.3 Results and discussion . . . . . . . . . . . . . . . . . . . 84

5.3.1 Block copolymer induced structure control in hybrid bulk assemblies . . . . . 84

5.3.2 Inorganic thin films with controlled pore size and porosity . . . . . . . . . . 87

5.3.3 Highly ordered network morphologies in silica-based thin films . . . . . . . 91

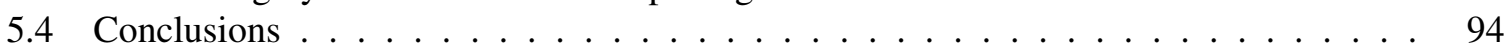

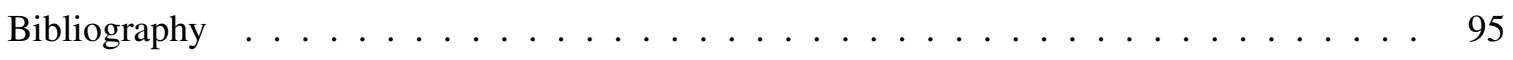

6 Crystal growth in block copolymer-derived mesoporous $\mathrm{TiO}_{2} \quad 99$

6.1 Introduction . . . . . . . . . . . . . . . . . . . . . . 99

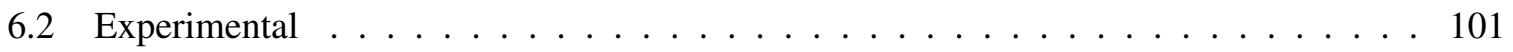

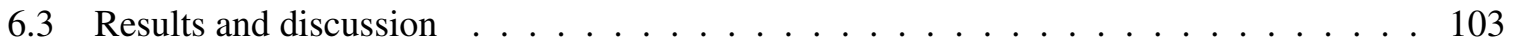

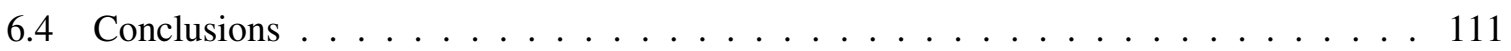

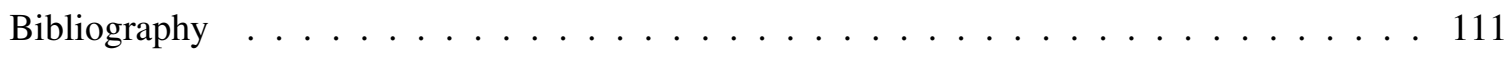

7 Thin film processing of block copolymer structure-directed inorganic materials $\quad 117$

7.1 Introduction . . . . . . . . . . . . . . . . . . . . . . . 117

7.2 Experimental . . . . . . . . . . . . . . . . . . . . . . . 119

7.3 Results and discussion . . . . . . . . . . . . . . . . . . . . . 120

7.4 Conclusions . . . . . . . . . . . . . . . . . . . . . . . . . . . . . . . . . 129

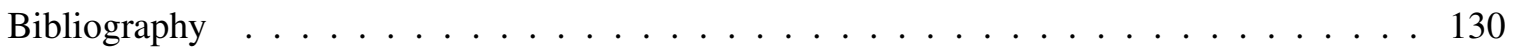

8 Tunable mesoporous Bragg reflectors based on block copolymer self-assembly $\quad 133$

8.1 Introduction . . . . . . . . . . . . . . . . . . . . . 133

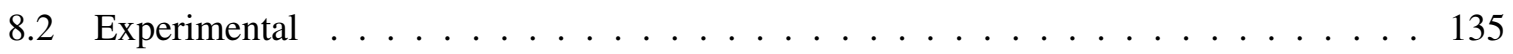

8.3 Results and discussion . . . . . . . . . . . . . . . . . . 136

8.4 Conclusions . . . . . . . . . . . . . . . . . . . . . . . . 142

Bibliography . . . . . . . . . . . . . . . . . . . 142

9 Dye-sensitised solar cell based on a three-dimensional photonic crystal 147

9.1 Introduction . . . . . . . . . . . . . . . . . . . . . . . . . 147

9.2 Experimental . . . . . . . . . . . . . . . . . . . . . . . . . . . . . . . . . 149

9.3 Results and discussion $\ldots \ldots \ldots \ldots$

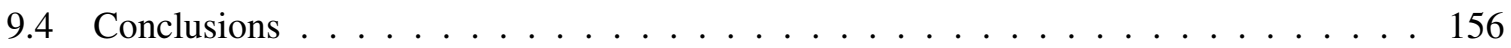

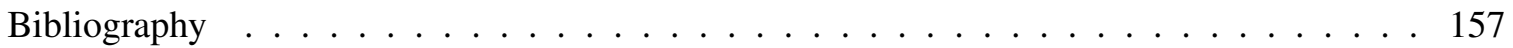

10 Block copolymer-assembled antireflective coatings with self-cleaning properties $\quad 161$

10.1 Introduction . . . . . . . . . . . . . . . . . . . . . 161

10.2 Experimental . . . . . . . . . . . . . . . . . . . 163

10.3 Results and discussion . . . . . . . . . . . . . . . . . . . . . . . . 164

10.4 Conclusions . . . . . . . . . . . . . . . . . . . . . . . . . . . . . . . . 174

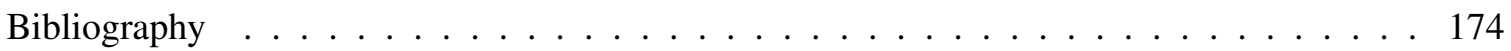

$\begin{array}{ll}11 \text { Conclusions } & 179\end{array}$

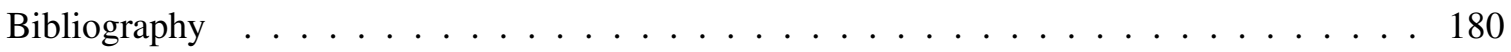

Curriculum vitae of Dr. Stefan Guldin $\quad 183$ 


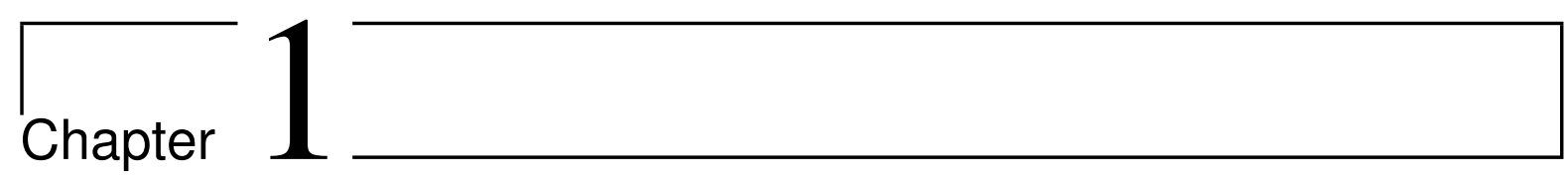

\title{
Self-assembly of soft matter
}

\author{
Molecular self-assembly is a common principle of structure formation in natu- \\ ral and synthetic materials. This chapter aims to explain the underlying driving \\ forces, from a broad perspective to the specific materials systems used in this \\ work.
}

Self-assembly is an enticing concept for anyone concerned about how to design and fabricate a materials structure. In a broad sense, the term defines formation processes which are reversible and driven by the cooperative assembly of predefined components into an ordered superstructure [1]. Assembly may occur between molecules, i.e. intermolecular or intramolecular in the form of folding along certain parts of a macromolecule. Furthermore, one may distinguish between static and dynamic self-assembly processes [2]. The former is used for systems that are, once formed, at a global or local equilibrium and therefore stable. Dynamic self-assembly, in contrast, is unstable and only occurs during the dissipation of energy, i.e. in self-organised systems like molecular motors or biological cells.

The driving forces for self-assembly are in general weak, non-covalent, intermolecular forces like van der Waals, electrostatic or hydrophobic interactions as well as coordination or hydrogen bonds. Beyond the molecular level, gravitational attraction, external electromagnetic fields, magnetic, capillary and entropic interactions may be other driving forces for structure formation of mesoscopic or macroscopic objects. The energy dissipated by intermolecular or mesoscopic forces is generally comparable to the thermal energy that allows the system to reach its thermodynamic equilibrium through fluctuations in position and orientation. 


\subsection{Self-assembly in nature}

Figure 1.1 shows three widely recognised examples of static self-assembly occurring in nature: lipid bilayer formation, protein folding and double helix formation of complementary strands of deoxyribonucleic acid (DNA). Phospholipids are the common building blocks for lipid bilayers, shown in Figure 1.1a-d. The macromolecules consist of a polar, hydrophilic head and two apolar, hydrophobic tails. In an aqueous medium, ensembles of phospholipids self-assemble into micellar or bilayer morphologies due to the energetically unfavourable interaction between their apolar tails and the surrounding water. The resulting configuration depends on the balance of energy costs for the exposure of hydrophobic tails at the bilayer's edges as compared to the curvature of the membrane into a closed spherical shape [3]. Lipid bilayers are of central importance in their function as cell membranes in most living organisms.

Protein folding is another phenomenon in biology, where hydrophobicity effect plays a key role for structure formation. The elementary building blocks of proteins are amino acids, whose linear sequence is called the primary structure. Particular sections within the primary structure can be generally distinguished as hydrophobic or hydrophilic, illustrated in Figure 1.1e as black or white spheres. Similarly to phospholipid systems, the molecule is therefore amphiphilic and tends to minimise the number of contact points between the hydrophobic elements of the chain and the surrounding water. This results in intermolecular self-assembly, i.e. folding of the protein into structural motifs, such as $\alpha$-helices and $\beta$-sheets (secondary structure) and further assembly into an energy-minimal global three-dimensional arrangement
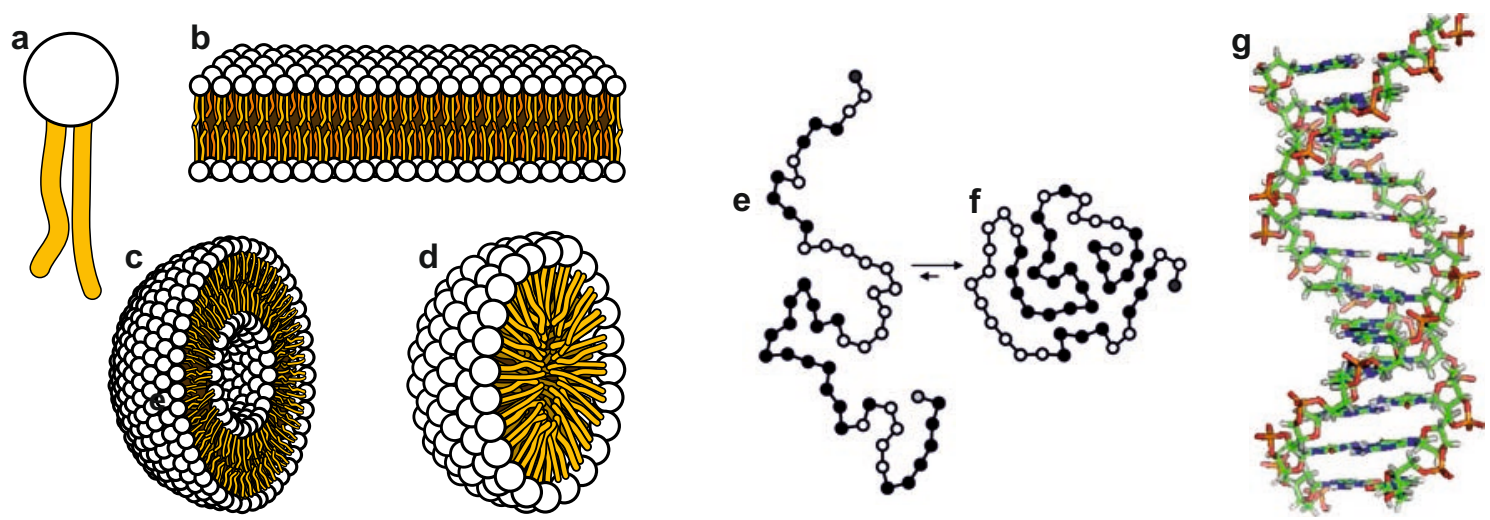

Figure 1.1: Self-assembly in biological systems. a) Phospholipid molecules consist of a polar, hydrophilic head and two apolar, hydrophobic tails. Depending on the energy-minimal configuration, ensembles of phospholipids in an aqueous environment self-assemble into b) lipid bilayer sheets, c) liposomes, d) and micelles. e) - f) Illustration of the protein folding mechanism. g) Double helix structure, formed by the self-assembly of two complementary strands of deoxyribonucleic acid. $\boldsymbol{a})-\boldsymbol{f}$ ) reproduced with permission from [4]. 
of the chain and its side groups (tertiary structure) [3].

A further prominent example for molecular self-assembly in nature is the formation of DNA into its tertiary structure, the double helix. The driving force for structure formation is the binding of each nucleotide component in a DNA strand with a complementary base pair. Adenine preferentially binds with thymine via two hydrogen bonds, guanine pairs with cytosine via three hydrogen bonds. Continuous hybridisation and melting enables the complementary molecular strands to reach their thermodynamic equilibrium in form of the double helix structure [5].

\subsection{Self-assembly in synthetic systems}

Advances in chemical synthesis of macromolecules as well as further understanding of structurefunction interplay in devices have fostered the field of soft nanotechnology over the last 20 years. In contrast to top-down materials fabrication approaches, such as photon or electron beam lithography, soft nanotechnology relies on the self-assembling nature of synthetically designed building blocks to enable morphology control on the nanometre-scale. The structure formation of homopolymers, block copolymers (BCP) and colloids was of particular importance for this work and will therefore be discussed in the following sections. Other materials systems employed in soft nanotechnology include emulsions [6, 7], synthetic DNA [5, 8, 9] as well as lyotropic $[10,11,12]$ and thermotropic liquid crystals [13, 14].

\subsubsection{Phase separation of polymer blends}

Homopolymers are macromolecules that consist of covalently bonded identical repeat units. The mixing behaviour of a blend of chemically different polymer chains can be well described by a mean free approach, introduced by Huggins [15] and Flory [16] in 1942. The FloryHuggins equation of the Gibbs free energy $G(T, P)$ for a blend of polymer A and B at temperature $T$ is known as:

$$
\Delta G_{\text {mix }}=\Delta H_{\text {mix }}-T \Delta S_{\text {mix }} .
$$

Accordingly, the thermodynamics of polymer blends is governed by the competing influence of enthalpic and entropic terms in the free energy of mixing. Flory and Huggins approached the change of enthalpy $\Delta H$ by a mean-field theory, taking into account possible interaction conformations of neighbouring monomers and their effects on the total enthalpy of the system in a lattice model: 


$$
\Delta H_{\text {mix }}=f_{A} f_{B} \chi_{\mathrm{AB}},
$$

where $f_{\mathrm{A}}$ and $f_{\mathrm{B}}=1-f_{\mathrm{A}}$ are the occupied volume fractions of polymer $\mathrm{A}$ and polymer $\mathrm{B}$, respectively. The dimensionless interaction parameter $\chi_{\mathrm{AB}}$ is defined as

$$
\chi=\chi_{\mathrm{AB}}=\frac{Z}{k_{\mathrm{B}} T}\left(w_{\mathrm{AB}}-\frac{1}{2}\left(w_{\mathrm{AA}}+w_{\mathrm{BB}}\right)\right)
$$

where $Z$ is the number of nearest-neighbour contacts, and $w_{\mathrm{AB}}, w_{\mathrm{AA}}$ and $w_{\mathrm{BB}}$ are the interaction energies per monomer between respective monomers ${ }^{\mathrm{i}}$.

The entropic change $\Delta S$ can be described by a combinatorial treatment, in which each monomer occupies a lattice site. The entropy is given by the number of possible arrangements $\Omega$ of the involved monomer units under the given boundary conditions

$$
S=k_{\mathrm{B}} \ln \Omega .
$$

Accordingly, the mixing-related gain in entropy due to an increase in spatial uncertainty is

$$
\Delta S_{\text {mix }}=-k_{\mathrm{B}}\left(\frac{f_{\mathrm{A}}}{N_{\mathrm{A}}} \ln f_{\mathrm{A}}+\frac{f_{\mathrm{B}}}{N_{\mathrm{B}}} \ln f_{\mathrm{B}}\right)
$$

with $N_{\mathrm{A}}$ and $N_{\mathrm{B}}$ for the degree of polymerisation of polymer $\mathrm{A}$ and polymer B, i.e. the number of subunits per chain.

Inserting the equations 1.2 and 1.5 into equation 1.1 leads to the Flory-Huggins description of the mixing behaviour of polymer blends:

$$
\frac{\Delta G_{\text {mix }}}{k_{\mathrm{B}} T}=\chi f_{\mathrm{A}} f_{\mathrm{B}}+\frac{f_{\mathrm{A}}}{N_{\mathrm{A}}} \ln f_{\mathrm{A}}+\frac{f_{\mathrm{B}}}{N_{\mathrm{B}}} \ln f_{\mathrm{B}} .
$$

Differential calculus of equation 1.6 allows to construct phase diagrams and predict the stability of blends as a function temperature and volume ratio, as shown in Figure 1.2c.

The tendency of long chain molecules to phase separate can be directly recognised from equations 1.2 and 1.5. While the enthalpic term $\Delta H$ in equation 1.2 is proportional to the number of monomers, the entropic term is decreased by a factor of $1 / N$ compared to a blend of unconnected monomers. A critical value of $\chi_{\mathrm{c}} \cdot N$ can be derived from the differentiation of $\Delta G\left(f_{\mathrm{A}}, N, \chi\right)$ for the symmetrical case of $N_{\mathrm{A}}=N_{\mathrm{B}}$. The condition $\chi \cdot N=2$ separates the situation in which mixtures of all compositions are stable $(\chi \cdot N<2)$ from the situation in which mixtures at some compositions will phase separate $(\chi \cdot N>2)$. Polymers are macromolecules

\footnotetext{
${ }^{\mathrm{i}}$ In practice $\chi$ may have more complex dependence on the temperature, for example $\chi=A / T+B$
} 
a
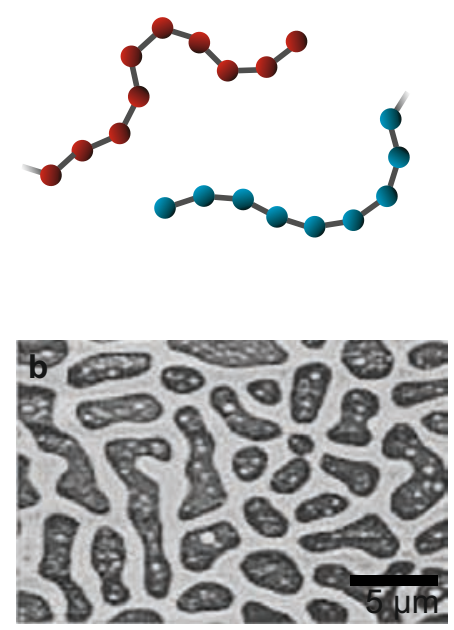

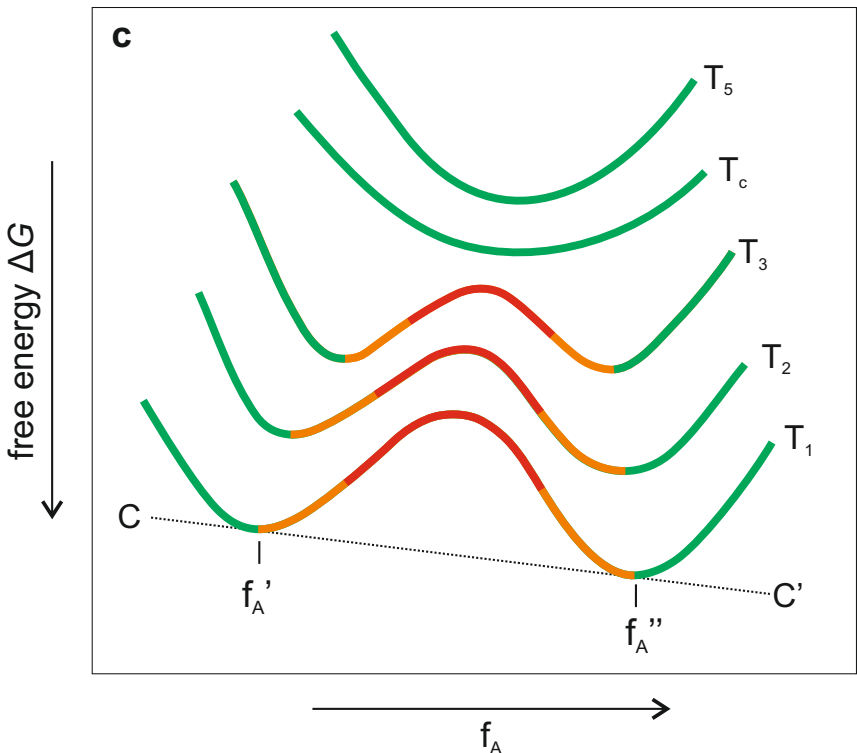

Figure 1.2: Phase separation of polymer blends. a) Schematic of two chemically distinct homopolymer chains. b) Atomic force microscopy image of a phase separated polymer film, consisting of poly(styrene) and poly(methyl methacrylate)-rich domains. c) Stability of a binary polymer blend as a function of temperature and volume composition. Green, orange and red regions indicate stable, metastable and unstable regions, respectively. Below a critical temperature $\mathrm{T}_{\mathrm{c}}$, the tie line $\overline{C C^{\prime}}$ through the local minima $(\partial \Delta G / \partial f)_{T, p}=0$ defines the coexisting compositions $f_{\mathrm{A}}^{\prime}$ and $f_{\mathrm{A}}^{\prime \prime}$, which separate the one-phase and two-phase region. For $\left(\partial^{2} \Delta G / \partial f^{2}\right)_{T, p} \leq 0$, the blend will spontaneously phase separate into domains with compositions $f^{\prime}$ and $f^{\prime \prime}$ via spinodal decomposition. In the metastable region (orange) phase separation is thermodynamically favourable but hindered by an energy barrier. The so-called binodal decomposition eventually occurs via nucleation and growth. b) Adapted with permission from [17]. Copyright 1997 American Chemical Society.

and typically have large values of $N$. Therefore even a very small positive value of $\chi$, stemming from a very weak repulsive monomer - monomer interaction will result in phase separation of the system $[3,18,19]$.

\subsubsection{Structure formation in block copolymer systems}

BCPs are a subclass of polymers where linear blocks of chemically different monomer units are covalently linked. Depending on the number and arrangement of constituting blocks, a variety of BCP architectures exist, e.g. diblock poly(A-block-B), triblock poly(A-b-B- $b-\mathrm{A}, \mathrm{A}-b-\mathrm{B}-b-$ C) and starblock copolymers [20]. Due to the covalent bonds of the chemically incompatible blocks, phase separation in BCP systems cannot extend to macroscopic length scales but is restricted to the dimensions of the polymer coil, typically in the range of $5-100 \mathrm{~nm}$.

The phase separation of a symmetric BCP A- $b$-B with $f_{\mathrm{A}}=f_{\mathrm{B}}$ into lamellar arrays of A- 
rich and B-rich domains is a direct consequence of the spatial constraint to phase separate on the macromolecular length scale. Yet, the theoretical description of the BCP phase behaviour in the melt has to take additional contributions into account which affect the lamellar spacing $d$. The enthalpic term, favouring the minimisation of interfacial energy, is now counterbalanced by free energy contributions that arise from the maximisation of the chains conformational entropy and, thus, oppose stretching of the chains. This entropic force for an ideal chain can be expressed by a Hookian term, reflecting the chains elasticity $\mathbf{F}_{\mathrm{el}}=\left(3 k_{\mathrm{B}} T\right) /\left(N a^{2}\right) \mathbf{R}$, where $\mathbf{R}$ is the extended end-to-end distance of the chain, and $a$ is a monomer size scale that depends on the local structure of the chain [18].

The sum of interaction and elastic energies per copolymer chain in the strong segregation limit can be described as

$$
\frac{G_{\mathrm{LAM}}}{k_{\mathrm{B}} T}=\frac{\gamma \Sigma}{k_{B} T}+\frac{3(d / 2)^{2}}{2 N a^{2}} .
$$

The first term accounts for enthalpic interactions that are confined to the A - B interface, as a product of the interfacial area per chain $\Sigma$ and the interfacial tension $\gamma$, which can be estimated by $\gamma=\left(k_{\mathrm{B}} T / a^{2}\right) \sqrt{\chi}$ according to a classical theory of polymer-polymer interfaces [3]. $\Sigma$ correlates to the chains fundamental characteristics $N$ and $a$ through the volume filling condition $\Sigma d / 2=N a^{3}$ (assuming incompressibility).

By minimising Equation 1.7 with respect to $d$ follows an expression for the equilibrium lamellar period $\left(d_{0}\right)$ :

$$
d_{0} / 2 \sim a \chi^{1 / 6} N^{2 / 3}
$$

The optimum lamellar domain period therefore scales as the two-thirds power of the copolymer molecular weight, which is in good agreement with experimental results [21].

In contrast to the above described microphase separation, the energy per chain in a homogeneous disordered phase can be approximated by the A - B contact area:

$$
\frac{G_{\mathrm{DIS}}}{k_{\mathrm{B}} T} \approx \chi f_{\mathrm{A}} f_{\mathrm{B}} N=\chi N / 4
$$

As a direct consequence, the order-disorder transition (ODT) occurs at $G_{\mathrm{DIS}}=G_{\mathrm{LAM}}$, i.e. $N \cdot \chi_{\mathrm{AB}}=10.4$. This is consistent with a more accurate mean field approach, yielding $N \cdot \chi_{\mathrm{AB}}=$ 10.5.

The outlined theory is a simplification and describes microphase separation adequately in the so-called strong segregation limit $(\chi N>100)$, where the domains are essentially phasepure and the interfaces sharply defined [22]. In the intermediate segregation regime below $\chi N \sim 100$ the step-like interface blurs out. In the weak segregation limit the polymer concentration ultimately resembles a sinusoidal profile [23]. A unified self-consistent mean-field 
approach has been developed that describes the system well in all regimes of $\chi N$ [24].

Symmetric lamellar domains are in general planar as interface curvature would result in an enthalpic penalty. For a rising volume asymmetry of the blocks, this configuration becomes increasingly unfavourable as longer blocks are more strongly stretched than shorter ones. Curvature of the interface towards the smaller block leads to a reduction of the systems free energy, resulting in a change of morphology from the lamellar phase to a bicontinuous gyroid phase $(\mathrm{G})$, as shown in Figure 1.3a. Further increase of asymmetry causes the formation of an hexagonal arrangement of cylindrical domains (C), followed by a body-centred cubic arrangement of spheres (S), close-packed spheres (CPS) and ultimately a disordered phase for the compositional extremes. The unified self-consistent mean free theory allows to determine the resulting equilibrium morphology as a function of $f_{\mathrm{A}}$ and $\chi N$, shown in Figure
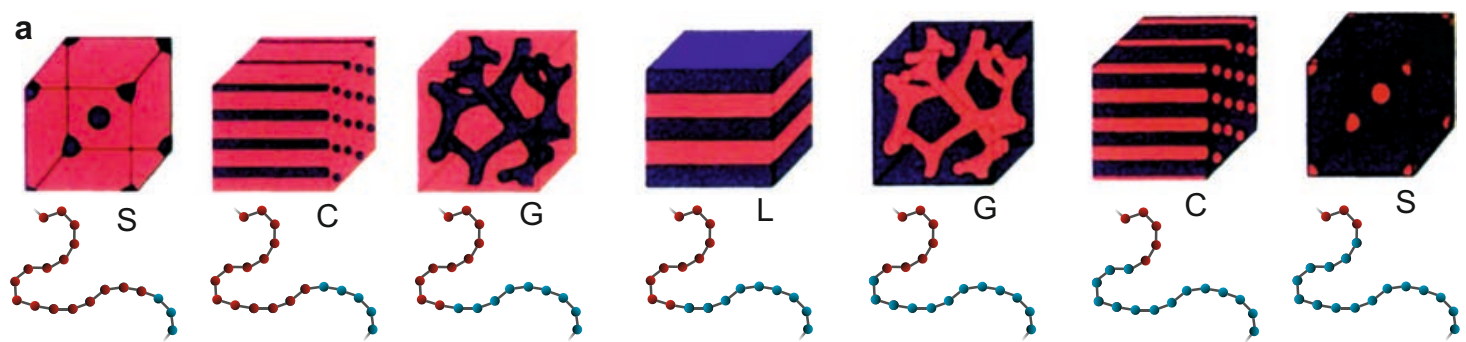

b

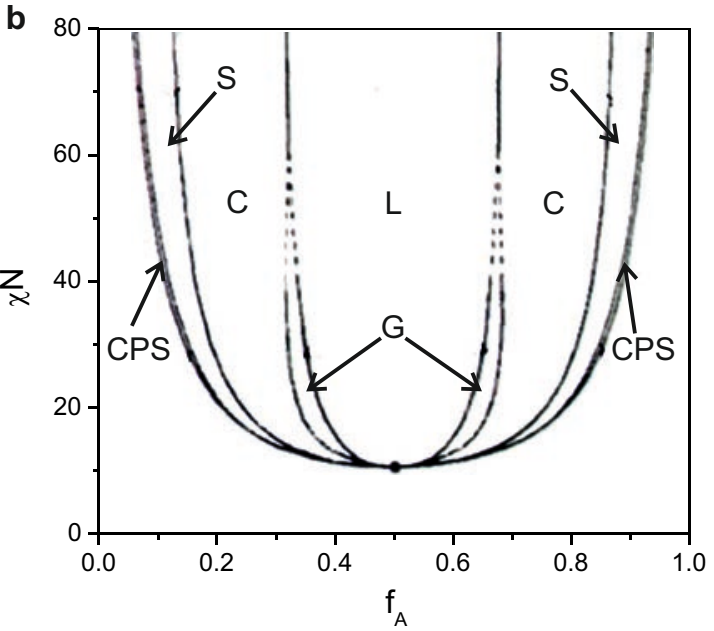

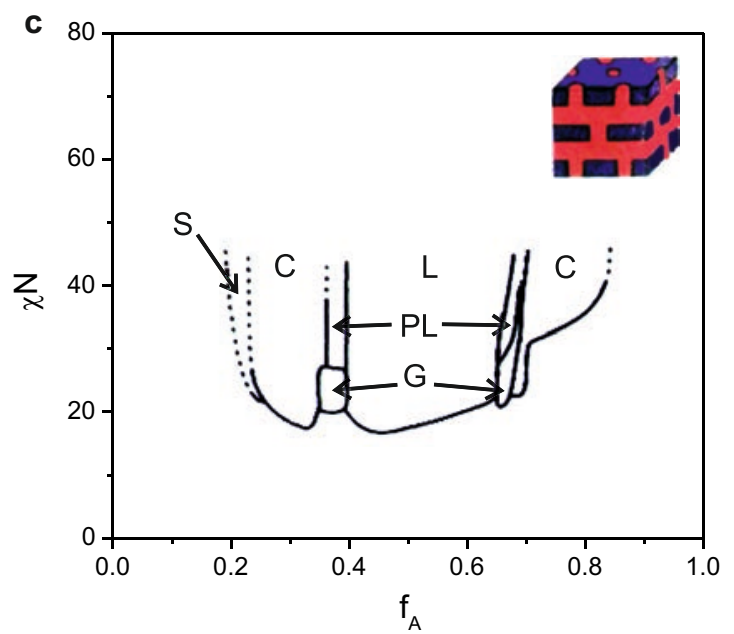

Figure 1.3: Phase separation of diblock copolymers. a) Schematic of diblock copolymer chain asymmetry and corresponding morphological arrangement after phase separation. Self-consistent mean-field theory predicts five arrangements at thermodynamic equilibrium with decreasing asymmetry: close-packed spheres (CPS, not shown), body-centred cubic arrangement of spheres (S), hexagonally packed cylinders $(\mathrm{C})$, the gyroid $(\mathrm{G})$ and lamellar phase $(\mathrm{L})$. b) The phase diagram can be parameterised by the volume fraction of block $\mathrm{A}\left(f_{\mathrm{A}}\right)$ and the combined parameter $\chi N$. c) Experimental data of BCP model systems like poly(isoprene- $b$-styrene) are in good agreement with calculations [25]. a) - c) Adapted with permission from [20]. Copyright 1999 American Institute of Physics. 
1.3b. This is in good agreement with correlated experimental data of amorphous BCP model systems like poly(isoprene- $b$-styrene), compared in Figure 1.3c. Additional phases like the perforated lamellae (PL) or double diamond (D) have been experimentally observed. These morphologies are kinetically long lived and metastable but not in thermodynamic equilibrium [26]. When extending the polymer architecture to three individual blocks, the interplay between $\mathrm{AB}-$, $\mathrm{BC}$ - and $\mathrm{AC}$-blocks gives rise to a ternary phase diagram with more than a dozen equilibrium morphologies. [20, 27, 28]

The ability of BCP self-assembly to control material arrangement on the nanometre scale into highly ordered morphologies is promising for technological applications. Many (opto)electronic devices such as batteries, fuel cells, photovoltaic cells and supercapacitors are highly interface sensitive and rely on efficient transport of charge and chemical reactants on the nanometre scale $[29,30,31]$. Numerous other photonic and biotechnological applications would greatly benefit from one-dimensional arrays [32, 33], interpenetrated networks $[34,35]$ or chiral material arrangements [36]. Yet, the previously discussed BCP self-assembly mechanisms are generally only valid for the phase separation of amorphous BCPs near thermodynamic equilibrium. Ensuring adequate experimental conditions in thin film processing is particular challenging due to the greatly increased evaporation kinetics and the interaction affinity of one of the blocks with the air or substrate interface [37, 38]. These issues may be circumvented by tuning the chemical properties of the substrate $[39,40]$, aligning the thin films in an electric field $[41,42]$ and post-treating the films in a controlled solvent atmosphere [43, 44].

A key challenge that remains for BCP-assembled device applications is the fact that accessible model systems do not exhibit the appropriate functionality. Widely used polymer blocks such as polystyrene, polyisoprene, polyethylene oxide, polymethyl methacrylate or polylactic acid lack photoactivity, conductivity or chemically stability to meet materials requirements in devices. In contrast device-active materials like conjugated polymers are typically stiff, often crystalline and exhibit low $N$ due to the greatly enhanced complexity of chemical synthesis, which poses major obstacles for exploiting BCP microphase separation in functional devices $[45,46,47]$.

\subsubsection{Block copolymer-directed co-assembly of inorganic material}

To-date technological benefit of BCP pattern generation has mostly been achieved by a sacrificial use of the self-assembly process as nanoscale templates to structure inorganic materials in thin films. After phase separation one of the blocks is selectively removed to form a porous template with a well-defined morphology. The pores can then be used as lithographic masks 
or refilled with a functional material of choice e.g. by solution impregnation, electrophoretic deposition or atomic layer deposition. This can be extended to a double templating approach in order to obtain the functional material in the complimentary morphology or for backfilling of the array with a second functional material. $[48,49,50]$. While pattern replication by sacrificial templating can be remarkable, fabrication is often complex due to inherent difficulties associated with the infiltration, such as clogging of the pores, the formation of blocking layers or insufficient thermal stability of the polymer mask.

A more direct route is the BCP-directed co-assembly of functional materials. In this case the macromolecules are used as structure-directing agents (SDAs) to guide the nanoscale assembly of inorganic material. After removal of the organic material by etching or high temperature calcination, the inorganic material then resembles the morphology imposed by the structure-directing host. Early approaches were based on low molecular weight cationic surfactants in combination with silicates derived by sol-gel chemistry $[10,11]$. Based on Coulomb interactions, the inorganic material was selectively incorporated into the surfactant's polar component. After calcination the silicates exhibited a highly ordered pore structure on the $1-10 \mathrm{~nm}$ length scale as a direct consequence of the liquid crystalline array formation of the organic SDA. This concept was subsequently extended to anionic [51] and non-ionic surfactants, i.e. BCPs [52]. The use of BCPs allowed to expand the attainable pore size through the increase in molecular weight and enforce phase separation due to high $\chi$ and $N$ of the amphiphilic couples. By employing poly(isoprene-block-ethylene oxide) of molecular weight $\geq 10 \mathrm{~kg} / \mathrm{mol}$, Wiesner and coworkers managed to overcome micellar material arrangements and reported hybrid morphologies which closely resembled BCP phases at thermodynamic equilibrium, such as lamellae and hexagonally packed cylinders [53].

Several conditions have to be fulfilled in order to reach BCP phase behaviour in a coassembly approach:

1. Intermolecular forces of the introduced inorganic material, such as Coulomb interactions and hydrogen bonding, need to be selective with one of the BCP's blocks [54].

2. The incorporated inorganic sol has to be small relative to the radius of gyration of the polymer chain to allow incorporation of guest material without perturbing the polymer's chain conformation [55].

3. Microphase separation needs to occur on significantly faster time scales than the competing gelation process of the inorganic material, arising from condensation reactions of the typically prehydrolysed sol. This can be achieved by a sufficiently large $\chi N$ parameter and a stabilised nanoparticle sol [56]. 
4. The solvent system has to dissolve all material components and enable even and slow evaporation of the volatile species [57].

Figure 1.4a shows a schematic illustration of the co-assembly process. Intimate mixing on the molecular level of the inorganic component with one of the polymer blocks allows the build-up of a two-domain system with well-defined interfaces [59]. In the example of Figure $1.4 \mathrm{~b}$ one domain is purely PI, opposing a domain consisting of PEO and aluminosilicate sol [58]. The resulting morphologies can be finely tuned by the volume ratio of PEO and inorganic material in this ternary materials system. Yet, the system can be treated as quasi-binary due to the intimate mixing of PEO and inorganic sol. The ternary system therefore differs from a $\mathrm{BCP}$ system with three independent blocks, such as poly(isoprene- $b$-styrene- $b$-ethylene oxide) [28].

A crucial requirement for the final conversion of the hybrid assembly into a purely inorganic array of high structural order is the stability of the inorganic morphology upon removal of the SDA. The guest material therefore needs to form a continuous network which requires to swell the hybrid assembly into the so-called dense nanoparticle regime [58, 60]. Typically, the inorganic guest outweighs the volume fraction of the polymer block it mixes with. While
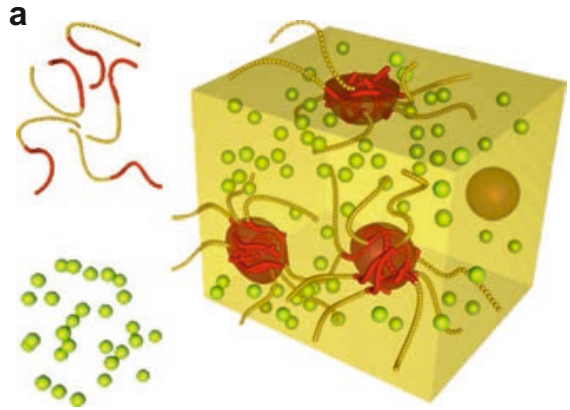

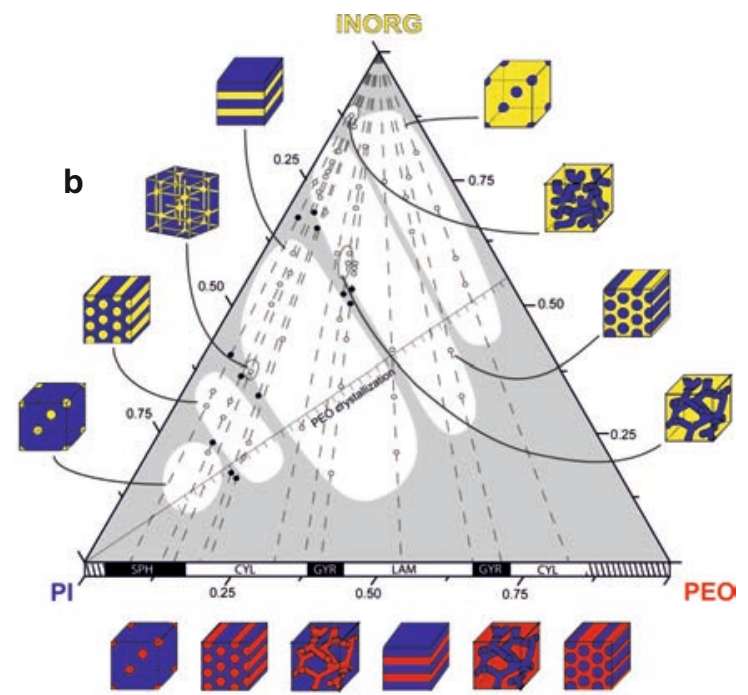

Figure 1.4: Block copolymer-directed co-assembly of inorganic material. a) Typically, an amphiphilic BCP and inorganic material in form of a sol are mixed in solution. Upon the evaporation of solvent, the inorganic sol resides in the hydrophilic block due to attractive intermolecular forces. The spatial arrangement of the inorganic guest material is therefore guided by the microphase separation process of the $\mathrm{BCP}$ host. b) The resulting hybrid morphologies can be mapped in a ternary phase diagram which is parameterised by the volume fractions of organic hydrophobic (PI), organic hydrophilic (PEO) and inorganic component (INORG). b) Reprinted with permission from [58]. Copyright 2009 American Chemical Society. 
this is now common practice in experiments, theoretical models still fall short of such high nanoparticle loadings $[61,62]$. These are typically based on a combination of polymer selfconsistent field theory with density functional theory to account for the mixture of soft, flexible chains with hard interdispersed spheres.

\subsubsection{Colloidal self-assembly}

The self-assembly of colloidal spheres presents another attractive platform for soft nanotechnology, in particular because colloids can crystallise into highly ordered 2D or 3D arrays.

\section{Colloidal interactions}

Colloid-colloid interactions in solution originate from a variety of attractive or repelling intermolecular forces. On the shortest length scale the impossibility of spatial overlap between hard spheres leads to repulsive excluded-volume interactions. Furthermore, entropic forces arise in dense concentrations due to the system's tendency to raise its conformational entropy. In addition, dipole-dipole interactions give rise to van der Waals forces between colloids, as schematically shown in Figure 1.5a. These are prevalent even for particles that have no permanent dipole due to fluctuations in electron density. A temporary dipole can induce a dipole in nearby particles, leading to a short-range attraction. For macroscopic spheres with known material distribution, the overall force can be calculated as the sum of all interacting pairs:

$$
U(r)=-\frac{A_{\mathrm{H}} R_{1} R_{2}}{6 r\left(R_{1}+R_{2}\right)},
$$

where $r$ is the distance between the spheres centre of mass, $R_{1}$ and $R_{2}$ are the radii of the spheres $\left(r \gg R_{1}, R_{2}\right)$ and $A_{\mathrm{H}}$ is the Hamaker constant. $A_{\mathrm{H}}$ is a material property and includes the number of atoms per unit volume and the coefficient of particle-particle pair interaction [63]. The Hamaker theory gives a good approximation but is not exact. A more accurate description, based on quantum field theory, has been developed by Lifshitz [64]. This model takes into account multi-body intermolecular interactions and the finite speed for electric field propagation in a surrounding medium, leading to a retardation effect of the dipole-dipole interaction for larger separations, where $U(r) \sim r^{-7}$. The surface of colloidal particles is typically charged, leading to electrostatic interactions as illustrated in Figure 1.5b. In an electrolyte solution the charges are screened by dissolved ions. These build-up a dense shell (Stern layer) and wider diffuse layer of opposite charges. The electrostatic potential $\Psi(x)$, which is generally given by the Poisson equation for a distribution of net charges, is now affected by the local density of counterions. This leads to the Poisson-Boltzmann equation: 


$$
\frac{\mathrm{d}^{2} \Psi}{\mathrm{d} x^{2}}=-\left(\frac{z e n_{0}}{\epsilon \epsilon_{0}}\right) \exp ^{-\frac{z e \Psi}{k_{\mathrm{B}} T}}
$$

where $\Psi$ is the electrostatic potential, $x$ is the distance from the spheres surface, $z \cdot e$ is the charge of the ions and $n_{0}$ is ionic concentration in the bulk solution. In the limit of small $\Psi$, Debye and Hückel approximated this term with [65]

$$
\psi(x)=\psi_{0} \exp ^{-\kappa x}
$$

where

$$
\kappa=\left(\frac{2 e^{2} n_{0} z^{2}}{\epsilon \epsilon_{0} k_{\mathrm{B}} T}\right)^{1 / 2} .
$$

The characteristic length $\kappa^{-1}$ is known as the Debye screening length. At distances $x \gg \kappa^{-1}$ electrostatic interactions become negligible. For monovalent salts of concentration $c(\mathrm{~mol} / \mathrm{l})$, the screening length is $\kappa^{-1}=0.304 c^{-0.5} \mathrm{~nm}$, thus $\kappa_{c=1}^{-1}=0.3 \mathrm{~nm}$ and $\kappa_{c=0.001}^{-1}=9.6 \mathrm{~nm}$.

Further intermolecular forces develop when the colloidal surfaces are grafted with polymer chains, shown in Figure 1.5c. In a good solvent a repelling force arises for approaching spheres due to an increase of polymer concentration and thus an osmotic pressure in the gap. In contrary, grafted polymers in a poor solvent result in an overall attraction. When polymers are not grafted onto the surface but dissolved in a colloidal solution, approaching spheres will exclude the solute within the gap. The local decrease in osmotic potential leads to a further attraction of the spheres, illustrated in Figure 1.5d.

These origins of intermolecular forces allow to finely tune colloidal interactions by:

- adjusting the ionic concentration of the electrolyte

- grafting of polymers onto the colloids surface (short vs. long-chain, low vs. high density, high vs. low solubility)

- addition of solutes on the intermediate length scale between colloids and solvent molecules

When repulsive interactions are dominant at all separation lengths, colloidal suspensions are stabilised and will not coagulate. For constituents of regular shape and narrow size distribution the evaporation of solvent, i.e. a dramatic increase in concentration can lead to a phase transformation from a disordered arrangement to a crystalline array. The regular packing of colloids into 2D or 3D lattices is a well-established tool in soft nanotechnology. Examples include the use of colloidal arrays as lithographic masks [66] or for a variety of photonic applications [67]. 

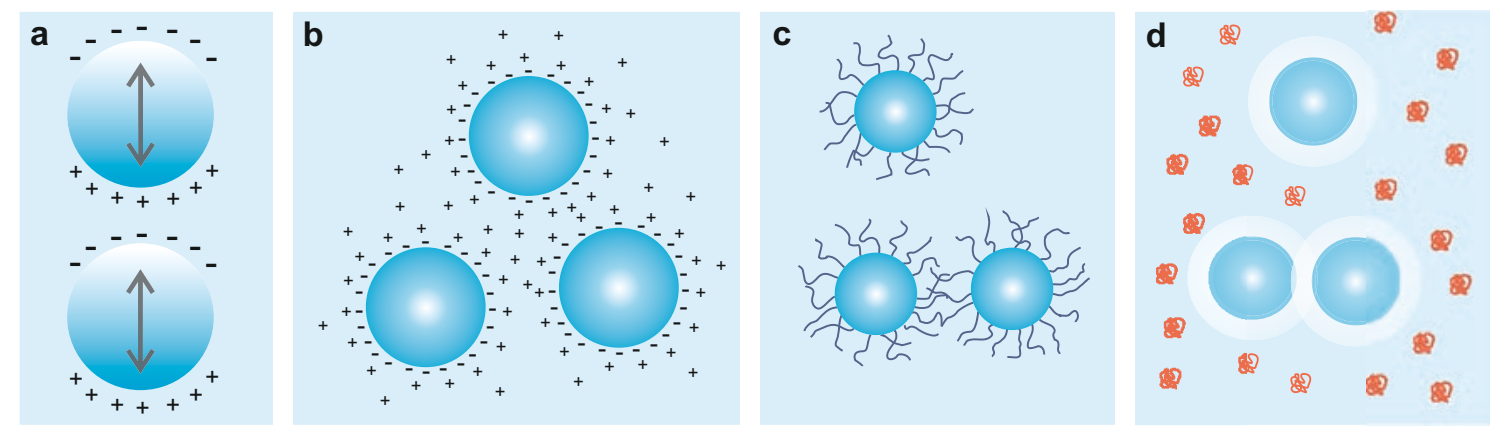

Figure 1.5: Colloidal interactions. a) Van der Waals forces (attractive, short range) arise from dipole-dipole interactions due to fluctuations in the electron density. b) Electrostatic interactions (repulsive, long range) stem from charge distribution on the colloid surface. The charge compensation can be described as a double layer, consisting of a dense shell (Stern layer) and wider diffuse layer of opposite charges. c) Grafted polymer on the colloids surface can lead to a repulsive force upon spatial overlap due to the osmotic pressure arising from the increased polymer concentration. In contrast, a poor solvent will lead to colloidal attraction. d) The addition of a solute on the intermediate length scale (i.e. homopolymers) leads to attractive forces due to the osmotic pressure which arises from the exclusion of this component in a depletion zone.

\section{Colloidal self-assembly}

While the origin of face-centred cubic (FCC) packing of spheres in 3D is still under debate [68], the formation mechanism in $2 \mathrm{D}$ is widely understood and is schematically shown in Figure 1.6 [69]. The ordering process is initiated once the solvent layer thickness is on the order of the particle diameter. Capillary forces arise from the liquid meniscus formed around the partially immersed sphere, which drives nearby spheres into close packing. By variation of particle size, concentration, electrolyte concentration, evaporation rate and surface tension, Denkov et al. substantiated that neither electrostatic repulsion nor van der Waals attraction are the main driving forces for the self-assembly [69]. The attractive horizontal force $F_{\mathrm{x}}$ arises from the slope of the three-phase contact line, that leads a variation of the surface tension force along the $\mathrm{x}$-axis [69]:

$$
F_{\mathrm{x}} \sim \frac{2 \pi \sigma r_{\mathrm{c}}^{2}}{L} \sin ^{2}\left(\psi_{\mathrm{c}}\right) \text { for } r_{\mathrm{c}} \ll L \ll\left(\frac{\sigma}{(\Delta \rho) g}\right)^{0.5} \text {, }
$$

where $\sigma$ is the surface tension of the liquid, $r_{\mathrm{c}}$ is the radius of the three-phase contact line at the sphere's surface and $\psi_{\mathrm{c}}$ is the mean meniscus slope angle at the contact line. The shape of the menisci is described by the Young-Laplace equation and depends on the distance between the colloids as well as the layer thickness $l_{0}$ and the particles wettability which is determined by the contact angle $\alpha$. An additional attractive term related to the higher hydrostatic pressure 
in the gas phase than the pressure in the liquid at $z>l_{0}$ can be neglected on the sub- $\mu \mathrm{m}$ length scale. Similar to the formation of a coffee stain, the pinning of the contact line at the edge of the droplet causes replenishing of the evaporated liquid by flux from the interior. This leads to convective transport of spheres to the outer edge of the suspension droplet, which drives further assembly (shown in Figure 1.6b) [70].

For the deposition of highly ordered colloidal arrays in 3D, the vertical evaporation-induced self-assembly method introduced by Colvin et al. has proven most effective [71]. A schematic is shown in 1.6c. The underlying formation mechanism that drives the colloids into a multilayered FCC lattice arrangement remains unclear. Norris et al. hypothesised that solvent flow through the interstitial spaces of closed-packed spheres play a major role in the evaporationinduced opal growth [68]. The flow, driven by the capillary suction of the menisci, may draw the spheres into the interstitial spaces, i.e. lead to the close packing in the thermodynamically favourable FCC lattice, even though the process takes place at non-equilibrium conditions. The origin of the high crystal quality in this vertical deposition technique may be due to the fact that concentration, rearrangement and lattice formation of the spheres all occurs simultaneously, which is fundamentally different to the horizontal capillary deposition described previously. Alternative methods for 3D colloidal crystallisation include field-induced, flowinduced and restricted-volume deposition [72]. Yet, the vertical deposition method is to-date considered to deliver the highest crystal quality [67].

While the assembly of sub- $\mu$ m sized colloidal spheres in a FCC crystal represents a 3D dielectric lattice, the architecture does not allow the formation of a full photonic band gap [73]. Infiltrating the structure with materials of refractive index $n>2.8$ and subsequently removing the spheres can result in an inverse opal structure that inhibits photon propagation in a certain frequency range [74]. This can be achieved by physical infiltration, i.e. impregnation of the structure with nanoparticles or molecular solutions as well as materials growth in the porous network by chemical reactants, such as atomic layer, chemical vapour, sol-gel or electrochemical deposition (chemical infiltration) [67]. Material infiltration does not only offer the possibility to increase the dielectric contrast but also allows the incorporation of active elements.

The stepwise assembly, drying and infiltration of colloidal arrays typically leads to significant volume contraction in the fabrication process, which can result in cracking and loss of long range order. A promising route to somewhat circumvent this problem is the simultaneous co-assembly of spheres and inorganic material. In this case, convective flow drives the guest material into the interstitial pores during the self-assembly process. Subsequent removal of the polymer spheres then leads directly to an inverse opal structure $[75,76]$.

The "Holy Grail" for self-assembled materials with enhanced optical response would be 

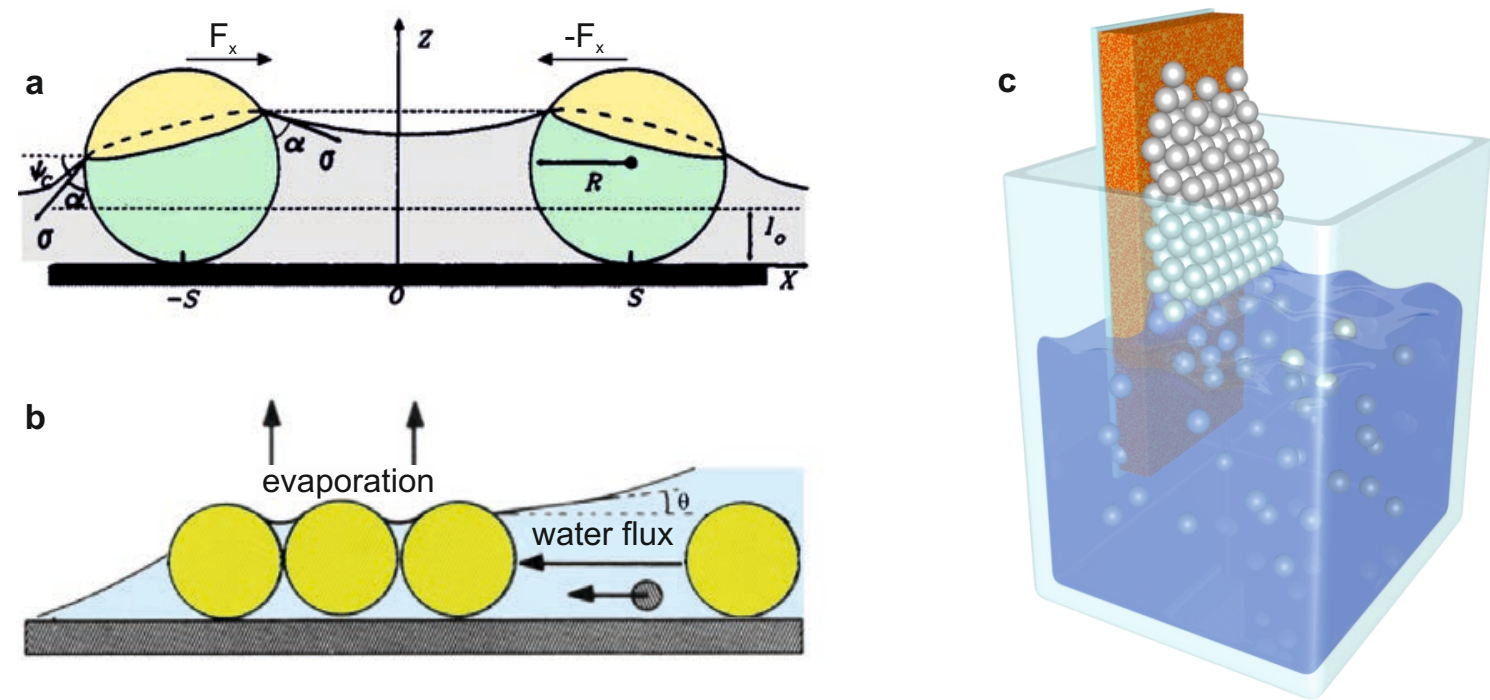

Figure 1.6: Colloidal interaction and evaporation induced self-assembly. a) Capillary forces lead to the attraction of nearby spheres when partially immersed in a liquid. b) Pinning of the contact line leads to convective transport of spheres to the edge of the suspension, which drives further assembly. c) The so-called evaporation-induced self-assembly method leads to the crystallisation of spheres into a three dimensional FCC lattice. Capillary forces are driving the spheres to the meniscus formed between the solution and the vertical substrate, whereas repulsive entropic and electrostatic forces between the spheres facilitate movement and therefore formation of the energetically favoured FCC structure. $\boldsymbol{a}$ ), $\boldsymbol{b}$ ): Adapted with permission from [69]. Copyright 1992 American Chemical Society.

the assembly of a diamond structure, i.e. a FCC crystal with a base of two constituents. From a photonic crystal perspective, this is widely seen as ideal structure, offering the possibility of a full photonic band gap in the visible range already for opaline arrays [67, 77]. Pathways have been proposed by using oppositely charged particles [78] or a binary mixture of colloidal spheres with selective etching properties [79, 80], but experimental realisations have not been shown.

\section{Bibliography}

[1] I. Hamley, "Nanotechnology with soft materials," Angewandte Chemie - Int. Ed., vol. 42, no. 15 , pp. $1692-1712,2003$.

[2] G. Whitesides and B. Grzybowski, "Self-assembly at all scales," Science, vol. 295, no. 5564, pp. 2418-2421, 2002.

[3] R. Jones, Soft condensed matter. Oxford University Press, 1st ed., 2002.

[4] Wikimedia - public domain graphic http://commons.wikimedia.org, 2011.

[5] N. Seeman, "Nanomaterials Based on DNA," Annual Review Of Biochemistry, vol. 79, pp. 65-87, 2010. 
[6] A. Imhof and D. Pine, "Ordered macroporous materials by emulsion templating," Nature, vol. 389, no. 6654, pp. 948-951, 1997.

[7] Y. Song, R. Garcia, R. Dorin, H. Wang, Y. Qiu, E. Coker, W. Steen, J. Miller, and J. Shelnutt, "Synthesis of platinum nanowire networks using a soft template," Nano Letters, vol. 7, no. 12, pp. 3650-3655, 2007.

[8] P. Rothemund, "Folding DNA to create nanoscale shapes and patterns," Nature, vol. 440, no. 7082, pp. 297-302, 2006.

[9] A. Pinheiro, D. Han, W. Shih, and H. Yan, "Challenges and opportunities for structural DNA nanotechnology," Nature Nanotechnology, vol. 6, no. 12, p. 763772, 2011.

[10] C. Kresge, M. Leonowicz, W. Roth, J. Vartuli, and J. Beck, "Ordered mesoporous molecular-sieves synthesized by a liquid-crystal template mechanism," Nature, vol. 359, no. 6397, pp. $710-712,1992$.

[11] J. Beck, J. Vartuli, W. Roth, M. Leonowicz, C. Kresge, K. Schmitt, C. Chu, D. Olson, E. Sheppard, S. McCullen, J. Higgins, and J. Schlenker, "A new family of mesoporous molecular-sieves prepared with liquid-crystal templates," Journal Of The American Chemical Society, vol. 114, no. 27, pp. 10834-10843, 1992.

[12] G. Attard, J. Glyde, and C. Goltner, "Liquid-crystalline phases as templates for the synthesis of mesoporous silica," Nature, vol. 378, no. 6555, pp. 366-368, 1995.

[13] T. Kato, N. Mizoshita, and K. Kishimoto, "Functional liquid-crystalline assemblies: selforganized soft materials," Angewandte Chemie - Int. Ed., vol. 45, no. 1, pp. 38-68, 2006.

[14] S. Sergeyev, W. Pisula, and Y. Geerts, "Discotic liquid crystals: a new generation of organic semiconductors," Chemical Society Reviews, vol. 36, no. 12, pp. 1902-1929, 2007.

[15] M. Huggins, "Thermodynamics properties of solutions of long-chain compounds," Annuals of the NY Academy of Sciences, vol. 43, pp. 1-32, 1942.

[16] P. Flory, "Thermodynamics of high polymer solutions," Journal of Chemical Physics, vol. 10, pp. 51-61, 1942.

[17] S. Walheim, M. Böltau, J. Mlynek, G. Krausch, and U. Steiner, "Structure formation via polymer demixing in spin-cast films," Macromolecules, vol. 30, no. 17, pp. 4995-5003, 1997.

[18] M. Rubinstein and R. Colby, Polymer physics. Oxford University Press, 1st ed., 2003.

[19] G. Strobl, The physics of polymers. Springer, 3rd ed., 2007.

[20] F. Bates and G. Fredrickson, "Block copolymers - designer soft materials," Physics Today, vol. 52, no. 2, pp. 32-38, 1999.

[21] T. Hashimoto, M. Shibayama, and H. Kawai, "Domain-boundary structure of styreneisoprene block co-polymer films cast from solution. 4. molecular-weight dependence of lamellar microdomains," Macromolecules, vol. 13, no. 5, pp. 1237-1247, 1980.

[22] E. Helfand and Z. Wasserman, "Block copolymer theory. 4. Narrow interphase approximation," Macromolecules, vol. 9, no. 6, pp. 879-888, 1976. 
[23] L. Leibler, "Theory of microphase separation in block co-polymers," Macromolecules, vol. 13, no. 6, pp. 1602-1617, 1980.

[24] M. Matsen and F. Bates, "Unifying weak- and strong-segregation block copolymer theories," Macromolecules, vol. 29, no. 4, pp. 1091-1098, 1996.

[25] A. Khandpur, S. Förster, F. Bates, I. Hamley, A. Ryan, W. Bras, K. Almdal, and K. Mortensen, "Polyisoprene-polystyrene diblock copolymer phase diagram near the order-disorder transition," Macromolecules, vol. 28, no. 26, pp. 8796-8806, 1995.

[26] M. Matsen and F. Bates, "Block copolymer microstructures in the intermediatesegregation regime," Journal Of Chemical Physics, vol. 106, no. 6, pp. 2436-2448, 1997.

[27] W. Zheng and Z. Wang, "Morphology of ABC triblock copolymers," Macromolecules, vol. 28, no. 21, pp. 7215-7223, 1995.

[28] T. Epps, E. Cochran, T. Bailey, R. Waletzko, C. Hardy, and F. Bates, "Ordered network phases in linear poly (isoprene-b-styrene-b-ethylene oxide) triblock copolymers," Macromolecules, vol. 37, pp. 8325-8341, 2004.

[29] Hagfeldt, A. and Grätzel, M., "Light-induced redox reactions in nanocrystalline systems," Chemical Reviews, vol. 95, no. 1, pp. 49-68, 1995.

[30] M. Grätzel, "Photoelectrochemical cells," Nature, vol. 414, no. 6861, pp. 338-344, 2001.

[31] A. Arico, P. Bruce, B. Scrosati, J. Tarascon, and W. Van Schalkwijk, "Nanostructured materials for advanced energy conversion and storage devices," Nature Materials, vol. 4, no. 5, pp. 366-377, 2005.

[32] M. Law, L. Greene, J. Johnson, R. Saykally, and P. Yang, "Nanowire dye-sensitized solar cells," Nature Materials, vol. 4, no. 6, pp. 455-459, 2005.

[33] R. Yan, D. Gargas, and P. Yang, "Nanowire photonics," Nature Photonics, vol. 3, no. 10, pp. 569-576, 2009.

[34] M. Hartmann, "Ordered mesoporous materials for bioadsorption and biocatalysis," Chemistry Of Materials, vol. 17, no. 18, pp. 4577-4593, 2005.

[35] X. Yang and J. Loos, "Toward high-performance polymer solar cells: the importance of morphology control," Macromolecules, vol. 40, no. 5, pp. 1353-1362, 2007.

[36] J. Pendry, “A chiral route to negative refraction," Science, vol. 306, no. 5700, pp. 13531355, 2004.

[37] T. Russell, G. Coulon, V. Deline, and D. Miller, "Characteristics of the surface-induced orientation for symmetric diblock PS/PMMA copolymers," Macromolecules, vol. 22, no. 12 , pp. 4600-4606, 1989.

[38] S. Anastasiadis, T. Russell, S. Satija, and C. Majkrzak, "Neutron reflectivity studies of the surface-induced ordering of diblock copolymer films," Physical Review Letters, vol. 62, no. 16, pp. 1852-1855, 1989.

[39] P. Mansky, Y. Liu, E. Huang, T. Russell, and C. Hawker, "Controlling polymer-surface interactions with random copolymer brushes," Science, vol. 275, no. 5305, pp. 14581460, 1997. 
[40] J. Heier, E. Kramer, S. Walheim, and G. Krausch, "Thin diblock copolymer films on chemically heterogeneous surfaces," Macromolecules, vol. 30, no. 21, pp. 6610-6614, 1997.

[41] K. Amundson, E. Helfand, X. Quan, S. Hudson, and S. Smith, "Alignment of lamellar block-copolymer microstructure in an electric-field. 2. Mechanisms of alignment," Macromolecules, vol. 27, no. 22, pp. 6559-6570, 1994.

[42] T. Thurn-Albrecht, J. DeRouchey, T. Russell, and H. Jaeger, "Overcoming interfacial interactions with electric fields," Macromolecules, vol. 33, no. 9, pp. 3250-3253, 2000.

[43] A. Knoll, A. Horvat, K. Lyakhova, G. Krausch, G. Sevink, A. Zvelindovsky, and R. Magerle, "Phase behavior in thin films of cylinder-forming block copolymers," Physical Review Letters, vol. 89, no. 3, 2002.

[44] H. Elbs, C. Drummer, V. Abetz, and G. Krausch, "Thin film morphologies of ABC triblock copolymers prepared from solution," Macromolecules, vol. 35, no. 14, pp. 5570$5577,2002$.

[45] U. Scherf, A. Gutacker, and N. Koenen, "All-conjugated block copolymers," Accounts of Chemical Research, vol. 41, no. 9, pp. 1086-1097, 2008.

[46] S. Darling, "Block copolymers for photovoltaics," Energy E Environmental Science, vol. 2, no. 12, pp. 1266-1273, 2009.

[47] M. Sommer, S. Hüttner, and M. Thelakkat, "Donor-acceptor block copolymers for photovoltaic applications," Journal Of Materials Chemistry, vol. 20, no. 48, pp. 10788-10797, 2010.

[48] M. Hillmyer, "Nanoporous materials from block copolymer precursors," in Block Copolymers II, vol. 190 of Advances in Polymer Science, pp. 137-181, 2005.

[49] M. Li and C. Ober, "Block copolymer patterns and templates," Materials Today, vol. 9, no. 9 , pp. 30-39, 2006.

[50] S. Darling, "Directing the self-assembly of block copolymers," Progress In Polymer Science, vol. 32, no. 10, pp. 1152-1204, 2007.

[51] Q. Huo, D. Margolese, U. Ciesla, P. Feng, T. Gier, P. Sieger, R. Leon, P. Petroff, F. Schüth, and G. Stucky, "Generalized synthesis of periodic surfactant inorganic compositematerials," Nature, vol. 368, no. 6469, pp. 317-321, 1994.

[52] S. Bagshaw, E. Prouzet, and T. Pinnavaia, "Templating of mesoporous molecular-sieves by nonionic polyethylene oxide surfactants," Science, vol. 269, no. 5228, pp. 1242-1244, 1995.

[53] M. Templin, A. Franck, A. DuChesne, H. Leist, Y. Zhang, R. Ulrich, V. Schädler, and U. Wiesner, "Organically modified aluminosilicate mesostructures from block copolymer phases," Science, vol. 278, no. 5344, pp. 1795-1798, 1997.

[54] P. Simon, R. Ulrich, H. Spiess, and U. Wiesner, "Block copolymer-ceramic hybrid materials from organically modified ceramic precursors," Chemistry Of Materials, vol. 13, no. 10, pp. 3464-3486, 2001.

[55] S. Warren, F. Disalvo, and U. Wiesner, "Nanoparticle-tuned assembly and disassembly of mesostructured silica hybrids," Nature Materials, vol. 6, no. 2, pp. 156-U23, 2007. 
[56] M. Stefik, S. Wang, R. Hovden, H. Sai, M. Tate, D. Muller, U. Steiner, S. Gruner, and U. Wiesner, "Networked and chiral nanocomposites from ABC triblock terpolymer coassembly with transition metal oxide nanoparticles," Journal Of Materials Chemistry, vol. 22, no. 3, pp. 1078-1087, 2012.

[57] M. Stefik, J. Song, H. Sai, M. Orilall, S. Guldin, P. Boldrighini, U. Steiner, S. Gruner, and U. Wiesner, "Ordered mesoporous titania from highly amphiphilic block copolymers: tuned solution conditions enable morphology control." manuscript in preparation.

[58] B. Garcia, M. Kamperman, R. Ulrich, A. Jain, S. Gruner, and U. Wiesner, "Morphology diagram of a diblock copolymer-aluminosilicate nanoparticle system," Chemistry Of Materials, vol. 21, no. 22, pp. 5397-5405, 2009.

[59] S. De Paul, J. Zwanziger, R. Ulrich, U. Wiesner, and H. Spiess, "Structure, mobility, and interface characterization of self-organized organic-inorganic hybrid materials by solidstate NMR," Journal Of The American Chemical Society, vol. 121, no. 24, pp. 57275736, 1999.

[60] A. Jain and U. Wiesner, "Silica-type mesostructures from block copolymer phases: formation mechanism and generalization to the dense nanoparticle regime," Macromolecules, vol. 37, no. 15, pp. 5665-5670, 2004.

[61] R. Thompson, V. Ginzburg, M. Matsen, and A. Balazs, "Predicting the mesophases of copolymer-nanoparticle composites," Science, vol. 292, no. 5526, pp. 2469-2472, 2001.

[62] K. Hur, R. Hennig, F. Escobedo, and U. Wiesner, "Mesoscopic structure prediction of nanoparticle assembly and coassembly: theoretical foundation," Journal Of Chemical Physics, vol. 133, no. 19, 2010.

[63] H. Hamaker, “The London - van der Waals attraction between spherical particles," Physica, vol. 4, pp. 1058-1072, 1937.

[64] E. Lifshitz, "The theory of molecular attractive forces between solids," Soviet Physics Jetp-USSR, vol. 2, no. 1, pp. 73-83, 1956.

[65] P. Debye and E. Hückel, "The theory of electrolytes I. The lowering of the freezing point and related occurrences," Physikalische Zeitschrift, vol. 24, pp. 185-206, 1923.

[66] J. Zhang, Y. Li, X. Zhang, and B. Yang, "Colloidal self-assembly meets nanofabrication: from two-dimensional colloidal crystals to nanostructure arrays," Advanced Materials, vol. 22, no. 38, pp. 4249-4269, 2010.

[67] J. Galisteo-Lopez, M. Ibisate, R. Sapienza, L. Froufe-Perez, A. Blanco, and C. Lopez, "Self-assembled photonic structures," Advanced Materials, vol. 23, no. 1, pp. 30-69, 2011.

[68] D. Norris, E. Arlinghaus, L. Meng, R. Heiny, and L. Scriven, "Opaline photonic crystals: how does self-assembly work?," Advanced Materials, vol. 16, no. 16, pp. 1393-1399, 2004.

[69] N. Denkov, O. Velev, P. Kralchevsky, I. Ivanov, H. Yoshimura, and K. Nagayama, "Mechanism of formation of 2-dimensional crystals from latex-particles on substrates," Langmuir, vol. 8, no. 12, pp. 3183-3190, 1992. 
[70] R. Deegan, O. Bakajin, T. Dupont, G. Huber, S. Nagel, and T. Witten, "Capillary flow as the cause of ring stains from dried liquid drops," Nature, vol. 389, no. 6653, pp. 827-829, 1997.

[71] P. Jiang, J. Bertone, K. Hwang, and V. Colvin, "Single-crystal colloidal multilayers of controlled thickness," Chemistry Of Materials, vol. 11, no. 8, pp. 2132-2140, 1999.

[72] F. Marlow, M. Muldarisnur, P. Sharifi, R. Brinkmann, and C. Mendive, "Opals: status and prospects," Angewandte Chemie - Int. Ed., vol. 48, no. 34, pp. 6212-6233, 2009.

[73] J. Joannopoulos, S. Johnson, J. Winn, and M. R.D., Photonic crystals: molding the flow of light. Princeton University Press, 2nd ed., 2008.

[74] K. Busch and S. John, "Photonic band gap formation in certain self-organizing systems," Physical Review E, vol. 58, no. 3, Part b, pp. 3896-3908, 1998.

[75] Q. Meng, C. Fu, Y. Einaga, Z. Gu, A. Fujishima, and O. Sato, "Assembly of highly ordered three-dimensional porous structure with nanocrystalline $\mathrm{TiO}_{2}$ semiconductors," Chemistry Of Materials, vol. 14, no. 1, pp. 83-88, 2002.

[76] B. Hatton, L. Mishchenko, S. Davis, K. Sandhage, and J. Aizenberg, "Assembly of large-area, highly ordered, crack-free inverse opal films," Proceedings Of The National Academy Of Sciences Of The United States Of America, vol. 107, no. 23, pp. 1035410359, 2010.

[77] C. Lopez, "Materials aspects of photonic crystals," Advanced Materials, vol. 15, no. 20, pp. 1679-1704, 2003.

[78] M. Leunissen, C. Christova, A. Hynninen, C. Royall, A. Campbell, A. Imhof, M. Dijkstra, R. van Roij, and A. van Blaaderen, "Ionic colloidal crystals of oppositely charged particles," Nature, vol. 437, no. 7056, pp. 235-240, 2005.

[79] A.-P. Hynninen, J. Thijssen, E. Vermolen, M. Dijkstra, and A. van Blaaderen, "Selfassembly route for photonic crystals with a bandgap in the visible region," Nature Materials, vol. 6, no. 3, pp. 202-205, 2007.

[80] F. Garcia-Santamaria, C. Lopez, F. Meseguer, F. Lopez-Tejeira, J. Sanchez-Dehesa, and H. Miyazaki, "Opal-like photonic crystal with diamond lattice," Applied Physics Letters, vol. 79, no. 15, pp. 2309-2311, 2001. 


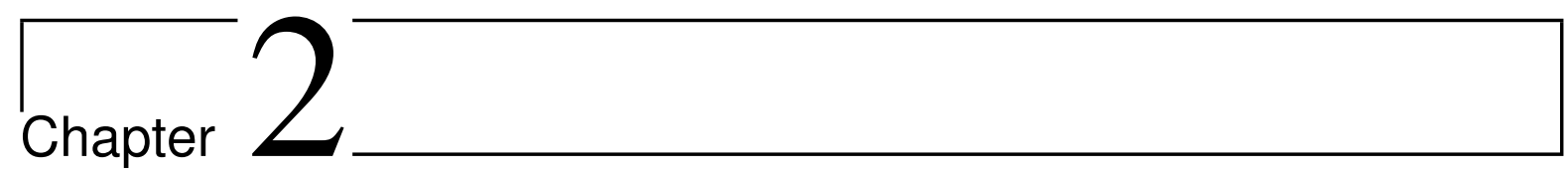

\section{Optical aspects of thin films and interfaces}

Much of the presented work has been devoted to the design of photonic structures by soft matter self-assembly. This chapter shall give a general introduction to the optics of thin films and interfaces and furthermore explain the main concepts and techniques that were used to analyse the experimental results.

\subsection{Propagation of light at an optical interface - reflection and refraction}

The propagation of electromagnetic (EM) waves in an optical medium is fully described by the four Maxwell equations, in the classical limit [1]. This leads to boundary conditions for the parallel $\left(^{\|}\right)$and perpendicular $\left({ }^{\perp}\right)$ field components which need to be met when a beam of light hits a planar interface between two homogeneous lossless dielectric media with permittivity $\epsilon_{1}$ and $\epsilon_{2}$, and permeability $\mu_{1}$, and $\mu_{2}$, as illustrated in Figure 2.1a [2].

$$
\begin{gathered}
\epsilon_{1} E_{1}^{\perp}=\epsilon_{2} E_{2}^{\perp} \\
B_{1}^{\perp}=B_{2}^{\perp} \\
\mathbf{E}_{1}^{\|}=\mathbf{E}_{2}^{\|} \\
\mathbf{B}_{1}^{\|} / \mu_{1}=\mathbf{B}_{2}^{\|} / \mu_{2} .
\end{gathered}
$$

These four equations 2.1-2.4 are the basis for the theory of reflection and refraction at optical interfaces. With a monochromatic planar incident wave of form $E_{\mathrm{i}}=E_{0, \mathrm{i}} \exp \left[i\left(\mathbf{k}_{\mathrm{i}} \mathbf{r}-\omega_{i} t\right)\right]$ one can derive from equation 2.3 the law of reflection [3]:

$$
\theta_{\mathrm{i}}=\theta_{\mathrm{r}}=\theta_{1},
$$


as well as Snell's law of refraction:

$$
n_{1} \sin \theta_{1}=n_{2} \sin \theta_{2}
$$

The relative amplitudes of incident, reflected and refracted waves depend on their polarisation with respect to the plane of incidence. Incoming light can be decomposed into orthogonal and linearly polarised waves, with E-field components perpendicular $\left(\mathbf{E}_{\mathrm{s}}\right)$ and parallel $\left(\mathbf{E}_{\mathrm{p}}\right)$ to the plane of incidence. Based on equations 2.3 and 2.4 the so-called Fresnel coefficients can be derived:

$$
\begin{aligned}
& r_{\mathrm{s}}=\frac{E_{\mathrm{r}, \mathrm{s}}}{E_{\mathrm{i}, \mathrm{s}}}=\frac{n_{1} \cos \theta_{1}-n_{2} \cos \theta_{2}}{n_{1} \cos \theta_{1}+n_{2} \cos \theta_{2}}, \\
& t_{\mathrm{s}}=\frac{E_{\mathrm{t}, \mathrm{s}}}{E_{\mathrm{i}, \mathrm{s}}}=\frac{2 n_{1} \cos \theta_{1}}{n_{1} \cos \theta_{1}+n_{2} \cos \theta_{2}}, \\
& r_{\mathrm{p}}=\frac{E_{\mathrm{r}, \mathrm{p}}}{E_{\mathrm{i}, \mathrm{p}}}=\frac{n_{2} \cos \theta_{1}-n_{1} \cos \theta_{2}}{n_{2} \cos \theta_{1}+n_{1} \cos \theta_{2}}, \\
& t_{\mathrm{p}}=\frac{E_{\mathrm{t}, \mathrm{p}}}{E_{\mathrm{i}, \mathrm{p}}}=\frac{2 n_{1} \cos \theta_{1}}{n_{2} \cos \theta_{1}+n_{1} \cos \theta_{2}} .
\end{aligned}
$$

Figure $2.1 \mathrm{~b}$ shows the corresponding reflectance $\mathcal{R}$ and refraction, i.e. transmittance $\mathcal{T}$, at a airglass interface with $\mathcal{R}_{\mathrm{s}, \mathrm{p}}=r_{\mathrm{s}, \mathrm{p}}^{2}$ and $\mathcal{T}_{\mathrm{s}, \mathrm{p}}=\left(n_{2} \cos \theta_{2} / n_{1} \cos \theta_{1}\right) \cdot t_{\mathrm{s}, \mathrm{p}}^{2}$, respectively. The Brewster angle $\theta_{\mathrm{B}}$ follows from $r_{\mathrm{p}}=0$ and Snell's law with $\theta_{\mathrm{B}}=\arctan \left(n_{2} / n_{1}\right)$.
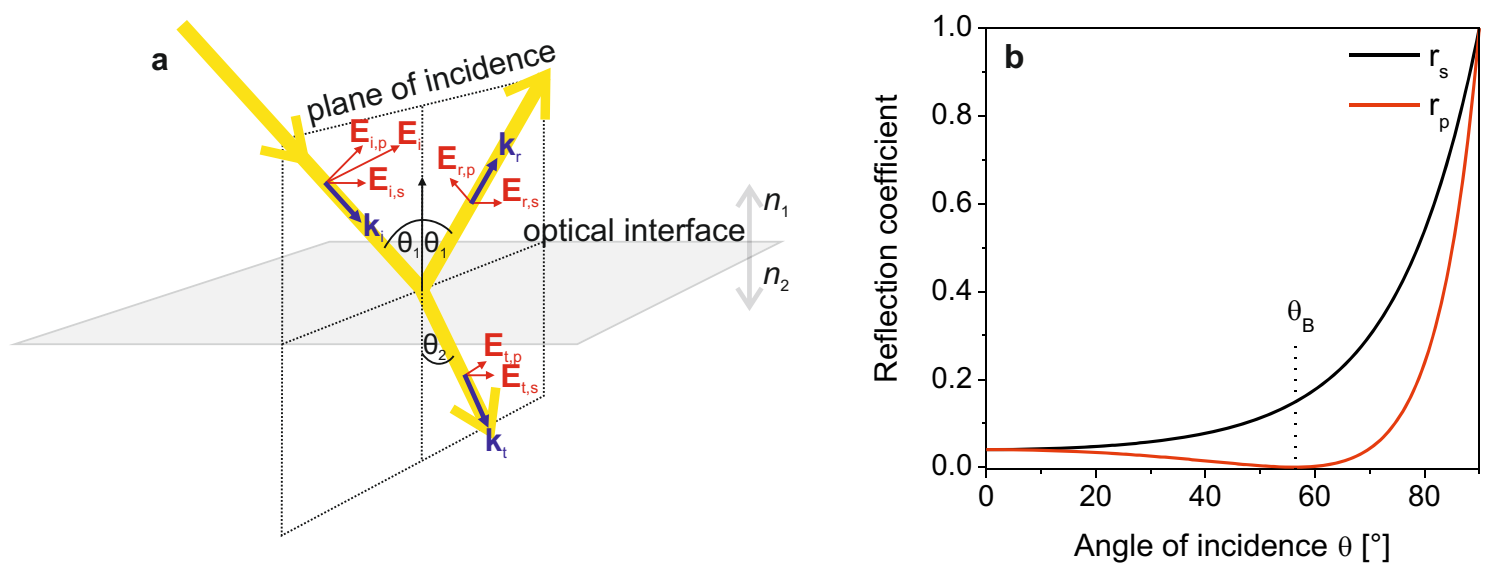

Figure 2.1: Reflection and refraction at an optical interface. a) Incident $\left(\mathbf{E}_{\mathbf{i}}, \mathbf{k}_{\mathrm{i}}\right)$, reflected $\left(\mathbf{E}_{\mathrm{r}}, \mathbf{k}_{\mathrm{r}}\right)$ and refracted $\left(\mathbf{E}_{\mathrm{t}}, \mathbf{k}_{\mathrm{t}}\right)$ EM waves at the interface between two homogeneous, isotropic and lossless dielectric media. The electric field can be decomposed into parallel $\left(\mathbf{E}_{\mathrm{p}}\right)$ and perpendicular $\left(\mathbf{E}_{\mathrm{s}}\right)$ components with respect to the plane of incidence. b) Reflection coefficients at an air-glass interface for parallel $\left(r_{\mathrm{p}}\right)$ and perpendicular $\left(r_{\mathrm{s}}\right)$ polarised incident light as a function of angle of incidence $(\theta)$. At the Brewster angle $\theta_{\mathrm{B}}=56.5^{\circ}$ all parallel polarised light is refracted $\left(r_{\mathrm{p}}=0\right)$, i.e. the reflected light is fully polarised perpendicularly to the plane of incidence. 


\subsection{Thin film interference}

The characteristic reflection of a thin film is the consequence of multiple beam interference. As shown in Figure 2.2a, the presence of two optical interfaces, each with its characteristic Fresnel coefficients for reflection and transmission, results in a division of the incident beam into a multitude of coherent waves. If the reflection coefficients are non-negligible, this results in a number of higher order reflected waves that all contribute to the colour appearance of the film, a characteristic of its thickness $\left(h_{\text {film }}\right)$ and refractive index $\left(n_{1}\right)$. The optical path difference $\Delta$ of two adjacent rays is given by:

$$
\Delta=2 n_{1} h_{\text {film }} \cos \theta_{1},
$$

with a corresponding phase shift $\phi_{m}=(m+1) \cdot(2 \pi / \lambda) \cdot \Delta$, where $m$ is the number of total internal reflections. $\mathbf{E}_{1}$ is therefore out-of-phase by $180^{\circ}$ compared to all higher order reflected waves $\left(\mathbf{E}_{2}, \mathbf{E}_{3}, \ldots\right)$, where $m$ is always an odd number. The resulting overall amplitude is then given by a geometric series, where $r_{i j}$ and $t_{i j}$ correspond to the Fresnel coefficients at the optical interface $i$ and $j$ denoted in order of the propagation direction of the ray [4]. This applies for perpendicular polarisation (with $r_{\mathrm{s}, i j}$ and $t_{\mathrm{s}, i j}$ ) and parallel polarisation (with $r_{\mathrm{p}, i j}$ and $t_{\mathrm{p}, i j}$ ).

$$
\begin{aligned}
\frac{E_{\mathrm{r}}}{E_{0}} & =r_{01}+t_{01} r_{12} t_{10} \exp ^{-2 i \phi} \sum_{n=1}^{\infty}(-1)^{n}\left(r_{12} r_{10}\right)^{n} \exp ^{-2 i n \phi} \\
& =r_{01}+\frac{t_{01} r_{12} t_{10} \exp ^{-2 i \phi}}{1+r_{10} r_{12} \exp ^{-2 i \phi}}=\frac{r_{01}+r_{12} \exp ^{-2 i \phi}}{1+r_{10} r_{12} \exp ^{-2 i \phi}},
\end{aligned}
$$

with $t_{01} t_{10}=\sqrt{1-r_{01}^{2}} \sqrt{1-r_{10}^{2}}=1-r_{01}^{2}$, assuming lossless dielectric media. The reflectivity of a thin film is then given by

$$
\begin{aligned}
\mathcal{R}=\frac{I_{\mathrm{r}}}{I_{0}} & =\frac{\left(r_{01}+r_{12} \exp ^{-2 i \phi}\right)\left(r_{01}+r_{12} \exp ^{2 i \phi}\right)}{\left(1+r_{01} r_{12} \exp ^{-2 i \phi}\right)\left(1+r_{01} r_{12} \exp ^{2 i \phi}\right)} \\
& =\frac{r_{01}^{2}+r_{12}^{2}+2 r_{01} r_{12} \cos 2 \phi}{1+r_{01}^{2} r_{12}^{2}+2 r_{01} r_{12} \cos 2 \phi} .
\end{aligned}
$$

For illustration, a computer-simulated colour reflection of a thin water film is shown in Figure 2.2. According to equation 2.11, the conditions for multiple beam interference depend on the film thickness $(h)$ and the angle of inclination $(\theta)$, here plotted in polar coordinates. 

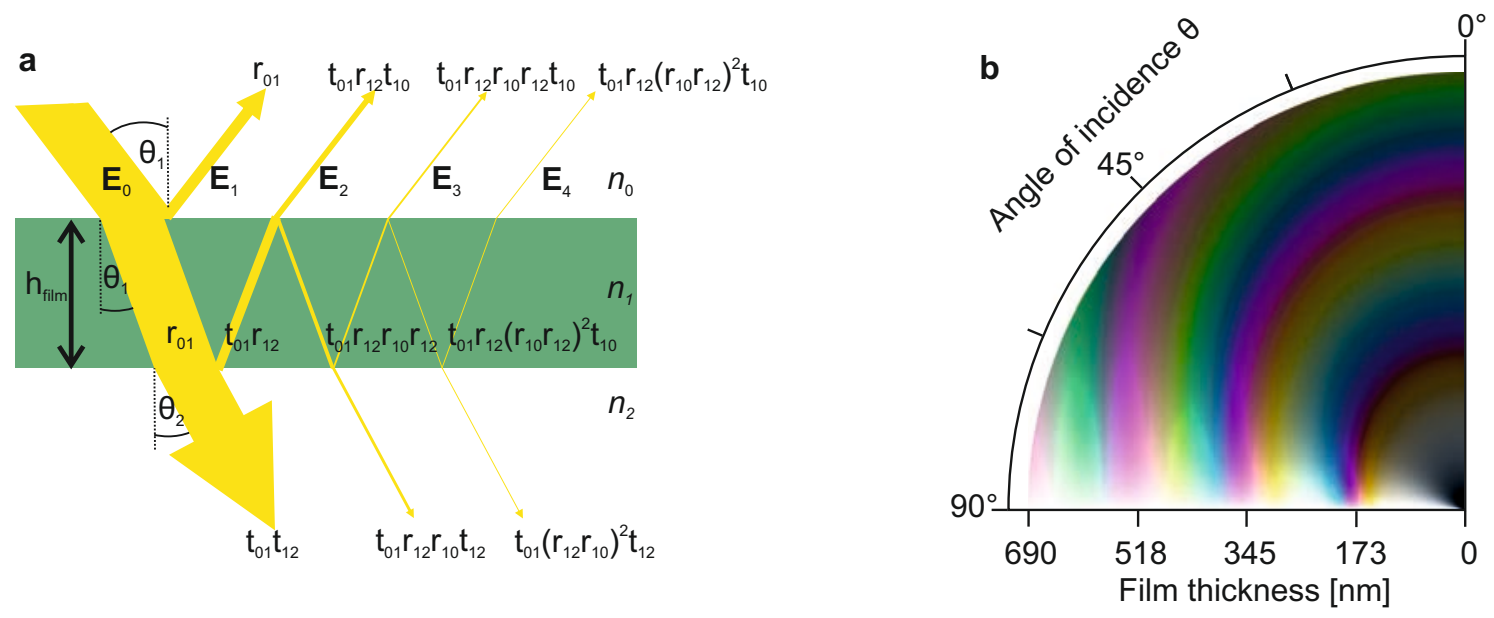

Figure 2.2: Thin film interference. a) The Fresnel equations determine the reflection and transmission coefficients of EM waves at each optical interface. The overall reflectivity of a thin film is therefore a superposition of a multitude of beams with different optical paths. b) Computersimulation illustrating the colour reflection of a thin water film plotted in polar coordinates as a function of the film thickness $(r)$ and the angle of incidence $(\theta)$ for unpolarised standard daylight illumination (D65). b) Reproduced with permission from [5].

\subsection{Model of multilayer reflectivity}

\subsubsection{Rouard's method}

The optical response of a multilayer stack, i.e. a layer sequence of thin films, results from the reflection and refraction characteristics at each of its optical interfaces. In analogue to the mathematical treatment for thin film interference, Rouard extended the method of summation from one layer with two interfaces to $k$ layers and $k+1$ interfaces in a step-wise procedure $[4,6]$. Following his technique the optical properties of the $k^{\text {th }}$ individual layer (in terms of reflection coefficient and accompanying phase change) at the bottom of the stack are replaced by a single surface with the appropriate characteristics. This is then incorporated into the calculation for the adjacent $(k-1)^{\text {th }}$ layer and so on. Based on equation 2.12 it follows for the amplitude $\rho_{k}$ and phase $\delta_{k}$ of the light reflected from the $k^{\text {th }}$ layer:

$$
\rho_{k} \exp ^{i \delta_{k}}=\frac{r_{k}+r_{k+1} \exp ^{-2 i \phi_{k}}}{1+r_{k} r_{k+1} \exp ^{-2 i \phi_{k}}} .
$$

The $k^{\text {th }}$ layer is subsequently treated as a surface with the effective Fresnel coefficient $\rho_{k} \exp ^{i \delta_{k}}$. The parameter $\rho_{k}$ can be calculated via the complex conjugate

$$
\rho_{k}^{2}=\frac{r_{k}^{2}+r_{k+1}^{2}+2 r_{k} r_{k+1} \cos 2 \phi_{k}}{1+r_{k}^{2} r_{k+1}^{2}+2 r_{k} r_{k+1} \cos 2 \phi_{k}} .
$$


$\delta_{k}$ can be written as [4]

$$
\delta_{k}=\eta_{k}-\xi_{k}
$$

with

$$
\tan \xi_{k}=\frac{r_{k+1} \sin 2 \phi_{k}}{r_{k}+r_{k+1} \cos 2 \phi_{k}}
$$

and

$$
\tan \eta_{k}=\frac{r_{k} r_{k+1} \sin 2 \phi_{k}}{1+r_{k} r_{k+1} \cos 2 \phi_{k}} .
$$

For the adjacent $(k-1)^{\text {th }}$ layer this results in

$$
\rho_{k-1} \exp ^{i \delta_{k-1}}=\frac{r_{k-1}+\rho_{k} \exp ^{-i \delta_{k}} \exp ^{-2 i \phi_{k-1}}}{1+r_{k-1} \rho_{k} \exp ^{-i \delta_{k}} \exp ^{-2 i \phi_{k-1}}}
$$

where $\rho_{k-1}$ and $\delta_{k-1}$ can be calculated accordingly following equations $2.15-2.18$. This process is repeated $k$-times to obtain the reflection coefficient of the overall system $\rho_{1}$.

\subsubsection{Transfer matrix method}

An alternative route to calculate the optical response of a multilayer stack is the so-called transfer matrix approach, which relates the electric and magnetic field at both interfaces of an optical element via a characteristic matrix [7, 8]. Assume an incoming beam at normal incidence and a single layer of optical thickness $l$ in-between two optical media, i.e. a sequence media 0 | layer $1 \mid$ media 2 . The boundary conditions, introduced in equations $2.1-2.4$, have to be fulfilled at interface 01 :

$$
\begin{aligned}
E_{0 \mathrm{i}}+E_{0 \mathrm{r}} & =E_{1 \mathrm{i}}+E_{1 \mathrm{r}} \\
n_{0} E_{0 \mathrm{i}}-n_{0} E_{0 \mathrm{r}} & =n_{1} E_{1 \mathrm{i}} n_{1} E_{1 \mathrm{r}},
\end{aligned}
$$

and at interface 12:

$$
\begin{aligned}
E_{1 \mathrm{i}} \exp ^{i k l}+E_{1 \mathrm{r}} \exp ^{-i k l} & =E_{t} \\
n_{1} E_{1 \mathrm{i}} \exp ^{i k l}-n_{1} E_{1 \mathrm{r}} \exp ^{-i k l} & =n_{2} E_{t} .
\end{aligned}
$$

This set of equations can be combined to

$$
\begin{aligned}
1+\frac{E_{0 \mathrm{r}}}{E_{0 \mathrm{i}}} & =\left(\cos k l-i \frac{n_{2}}{n_{1}} \sin k l\right) \frac{E_{t}}{E_{0 i}} \\
n_{0}-n_{o} \frac{E_{0 \mathrm{r}}}{E_{0 \mathrm{i}}} & =\left(-i n_{1} \sin k l+n_{2} \cos k l\right) \frac{E_{t}}{E_{0 i}},
\end{aligned}
$$


or equivalently in a matrix representation

$$
\left(\begin{array}{c}
1 \\
n_{0}
\end{array}\right)+\left(\begin{array}{c}
1 \\
-n_{0}
\end{array}\right) \frac{E_{0 \mathrm{r}}}{E_{0 \mathrm{i}}}=\left(\begin{array}{cc}
\cos k l & \left(-i / n_{1}\right) \sin k l \\
-i n_{1} \sin k l & \cos k l
\end{array}\right)\left(\begin{array}{c}
1 \\
n_{2}
\end{array}\right) \frac{E_{\mathrm{t}}}{E_{0 \mathrm{i}}} .
$$

For clarity, equation 2.26 can be written as

$$
\left(\begin{array}{c}
1 \\
n_{0}
\end{array}\right)+\left(\begin{array}{c}
1 \\
-n_{0}
\end{array}\right) r=M\left(\begin{array}{c}
1 \\
n_{2}
\end{array}\right) t
$$

where $r$ is the reflection coefficient, $t$ is the transmission coefficient and $M$ is the transfer matrix. The extension to the optical response of $N$-layers is then accordingly

$$
\left(\begin{array}{c}
1 \\
n_{0}
\end{array}\right)+\left(\begin{array}{c}
1 \\
-n_{0}
\end{array}\right) r=M_{1} M_{2} M_{3} \ldots M_{N}\left(\begin{array}{c}
1 \\
n_{2}
\end{array}\right) t=M_{\mathrm{tot}}\left(\begin{array}{c}
1 \\
n_{2}
\end{array}\right) t
$$

where $M_{\mathrm{tot}}$ is the product of the individual transfer matrices.

A multilayer stack of particular interest is a distributed Bragg reflector (DBR), which consists of an periodic sequence of high and low refractive index layers. Strong reflection arises when all reflected beams are of equal phase and therefore interfere constructively at the front surface. This is fulfilled for $n_{\text {low }} h_{\text {low }}=n_{\text {high }} h_{\text {high }}=\lambda / 4$. DBRs are therefore typically denoted as quarter-wave stacks [9]. The product of two adjacent transfer matrices is therefore

$$
\left(\begin{array}{cc}
0 & -i / n_{\text {low }} \\
-i n_{\text {low }} & 0
\end{array}\right)\left(\begin{array}{cc}
0 & -i / n_{\text {high }} \\
-i n_{\text {high }} & 0
\end{array}\right)=\left(\begin{array}{cc}
n_{\text {high }} / n_{\text {low }} & 0 \\
0 & -n_{\text {low }} / n_{\text {high }}
\end{array}\right) \text {, }
$$

and correspondingly for the overall optical element with $2 N$ layers

$$
M=\left(\begin{array}{cc}
n_{\text {high }} / n_{\text {low }} & 0 \\
0 & -n_{\text {low }} / n_{\text {high }}
\end{array}\right)^{N}=\left(\begin{array}{cc}
\left(n_{\text {high }} / n_{\text {low }}\right)^{N} & 0 \\
0 & \left(-n_{\text {low }} / n_{\text {high }}\right)^{N}
\end{array}\right) .
$$

The reflectance of the multilayer stack can then be obtained by solving equation 2.27 for $r$

$$
\mathcal{R}=|r|^{2}=\left[\frac{\left(n_{\text {high }} / n_{\text {low }}\right)^{2 N}-1}{\left(n_{\text {high }} / n_{\text {low }}\right)^{2 N}+1}\right]^{2}
$$


The position of the reflectance maximum can be calculated by geometric considerations [10]:

$$
m \lambda_{\text {max }}=2\left(h_{1} \sqrt{n_{1}^{2}-n_{0}^{2} \sin \theta_{i}}+h_{2} \sqrt{n_{2}^{2}-n_{0}^{2} \sin \theta_{i}}\right),
$$

where $h_{1,2}$ and $n_{1,2}$ are the layer thickness and refractive index of component 1 and 2, respectively and $m \in \mathbb{N}$.

To model multilayer structures in this work, Rouard's technique was implemented into a "MatLab" algorithm that was developed by Dr. Mathias Kolle [10]. An alternative method based on a transfer matrix algorithm was used for comparison [11]. The optical constants of the building blocks were determined individually by spectroscopic ellipsometry. Figure 2.3a shows example calculations for interference-based antireflective coatings (ARC) which consist of one or two layers on a glass substrate to allow for amplitude and phase matching [12]. Wavelength-dependent optical constants were used to account for the optical dispersion of the used materials. In Figure $2.3 \mathrm{~b}$ an optical model is presented for stacks of $\lambda / 4$ layers with alternating high and low refractive index layers on a glass substrate. An odd number of overall layers corresponds high refractive index layers situated at the air-stack and stack-substrate interfaces.
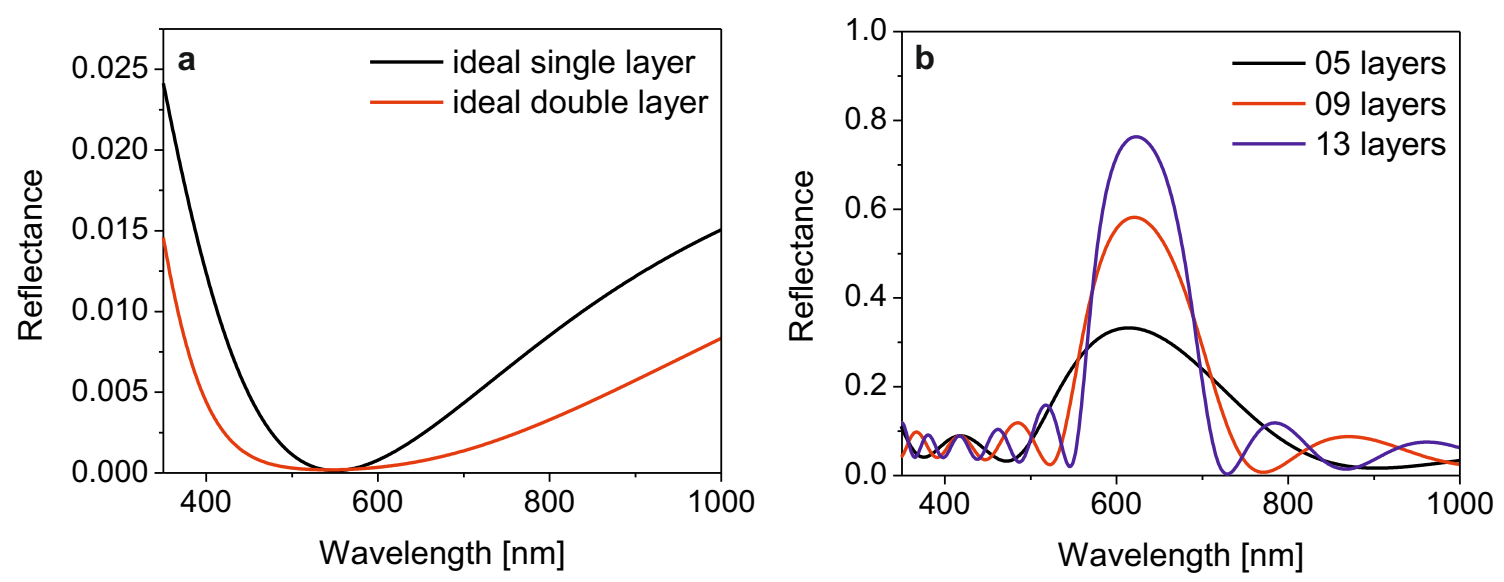

Figure 2.3: Modelling of multilayer interference. a) Calculated reflectance at normal incidence for interference-based antireflective coatings (ARC) optimised for $\lambda=550 \mathrm{~nm}$. An ideal single layer ARC yields $n_{\mathrm{ARC}}=1.22$ and $h_{\mathrm{ARC}}=112 \mathrm{~nm}$ to fulfill the amplitude and phase matching conditions on a glass substrate $\left(n_{\text {subs }}=1.50\right)$. The ideal double layer configuration is calculated for comparison, with $n_{\mathrm{ARC}_{\text {low }}}=1.11, h_{\mathrm{ARC}_{\text {low }}}=123 \mathrm{~nm}, n_{\mathrm{ARC} \text { high }}=1.36$ and $h_{\mathrm{ARC}_{\text {high }}}=101 \mathrm{~nm}$. b) Reflectance at normal incidence for multilayer stacks consisting of an odd number of alternating $\lambda / 4$ layers of high and low refractive index on a glass substrate, with $n_{\text {high }}=1.69, h_{\text {low }}=75 \mathrm{~nm}$, $n_{\text {low }}=1.41, h_{\text {high }}=130 \mathrm{~nm}$ and $n_{\text {subs }}=1.50$. 


\subsection{From periodic multilayer stacks to photonic crystals}

The model calculation presented in $2.3 \mathrm{~b}$ illustrates the change in optical response when extending the number of building blocks of a DBR. A further increase in the number of layers or contrast in refractive index ultimately leads to sharply defined, frequency-dependent reflection and transmission characteristics. The alternating sequence of high and low refractive index layers represents a dielectric lattice and can therefore be regarded as a one-dimensional (1D) photonic crystal. Similar to electron propagation in atomic crystals, the periodic modulation of the dielectric creates a potential landscape which determines the propagation directions of photons within the material as a function of frequency. In the case of high symmetries and dielectric contrast, a full three-dimensional photonic band gap can develop for a defined frequency range. This concept was first introduced by Yablonovitch [13] and John [14] and has become a very active field of research ever since $[15,16]$.

The main function, that contains all the information for a given dielectric system is the magnetic vector field $\mathbf{H}(\mathbf{r}, t)=\mathbf{H}(\mathbf{r}) e^{i \omega t}$. Based on the fact that macroscopic electromagnetism is governed by the four Maxwell equations, a so-called master equation can be deduced:

$$
\nabla \times\left(\frac{1}{\varepsilon(\mathbf{r})} \nabla \times \mathbf{H}(\mathbf{r})\right)=\frac{\omega^{2}}{c^{2}} \mathbf{H}(\mathbf{r})
$$

which is the analogon to the Schrödinger equation for the quantum mechanical consideration of an electron. See reference [17] for a comprehensive derivation.

The periodicity of the system is given by the permittivity $\varepsilon(\mathbf{r})=\varepsilon(\mathbf{r}+\mathbf{R})$ for all lattice vectors $\mathbf{R}$. It is essential that $\nabla \mathbf{H}=0$, i.e. the vector field has to be source- and sink-free as well as transverse. Assuming that the Maxwell operator $\hat{\Theta}=\nabla \times\left(\varepsilon(\mathbf{r})^{-1} \nabla \times\right)$ is a linear Hermitian operator, one can use a variational theorem to determine the normal modes and frequencies:

$$
E_{\mathrm{var}}=\frac{(\mathbf{H}, \hat{\Theta} \mathbf{H})}{\mathbf{H}, \mathbf{H}}
$$

The band structure functions $\omega_{n}(\mathbf{k})$ of a photonic crystal can therefore be exactly calculated $a b$ initio from $\varepsilon(\mathbf{r})$ by applying a computational scheme to solve the master equation for the magnetic modes of the photonic crystal under the given boundary conditions. Typical approaches are the finite difference time domain method [18] or the planar wave expansion method [19], which is presented for a 1D model system in reference [10]. Accordingly, the bandwidth of the fundamental reflection peak $\Delta \lambda_{\max }$ (full width at half max) for a photonic crystal-like DBR can be calculated [17]

$$
\Delta \lambda_{\max }=\frac{4 \lambda_{\mathrm{max}}}{\pi} \sin ^{-} 1\left(\frac{n_{\mathrm{high}}-n_{\mathrm{low}}}{n_{\mathrm{high}}+n_{\mathrm{low}}}\right) .
$$




\subsection{Optical properties of porous thin films}

In many different aspects, this work explores structure control in mesoporous materials on the $10 \mathrm{~nm}$ length scale. Conceptually, block copolymer (BCP) self-assembly offers precise control over the pore architecture, pore size and pore volume (i.e. porosity) in thin dielectric films. The optical properties of mesoporous materials are not simply a consequence of the constituent materials' crystal band structures, but are dependent on pore dimensions and volume on suboptical length scales. While generally the real and imaginary parts of the refractive index are an intrinsic property of the material, these can be finely tuned by adjusting the porosity as well as infiltration and blending of the mesostructure with other materials.

Metamaterials are a particular example where morphological arrangement on the subwave-length scale is of central importance to the resulting optical properties [20, 21]. The experimental realisation of a material with negative refractive index $n=\sqrt{\mu \varepsilon}<0$ typically requires negative permeability $\mu$ and the permittivity $\varepsilon$ in the same frequency range. This can be realised by a so-called split-ring resonator geometry, experimentally achieved for microwave to infrared frequencies $[22,23,24]$. Currently, access to visible frequencies is impeded by resolution limits in the fabrication process. Alternative approaches have been developed but their experimental realisation remains a major challenge [25]. An alternative concept is a chiral route to negative refraction [26]. In a chiral medium the refractive index is polarisationdependent. The band splitting of the resonant transverse modes leads to a range of frequencies, where the group velocity $v_{g}=\partial \omega / \partial k$ has an opposite sign to the phase velocity $v_{p}=\omega / k$, leading to $n<0$ for a certain polarisation. The validity of this concept has already been shown at terahertz frequencies [27]. However, like for other metamaterial concepts, such as splitring resonators, it proofs difficult to scale down the structural feature sizes to achieve negative refraction in the visible spectral range.

As outlined in Section 1.2.2 the self-assembly of BCP systems allows access to well defined nanoarchitectures. BCP morphologies have unit-cells far smaller than the wavelength of light, which makes this system a interesting platform for the bottom-up assembly of optical metamaterials. A possible materials route to BCP-templated arrays is illustrated in Figure 2.4, where the metal structure is fabricated in four steps: (1) BCP self-assembly in a thin film, (2) selective etching of one of the polymer blocks, (3) backfilling of the template with a metal, such as silver or gold and (4) selective etching of the residual polymer components [28]. Of particular relevance to optical applications are bicontinuous gyroid morphologies. For a diblock copolymer, the double gyroid consists of two network structures of block A separated by a complementary matrix of block B (Figure 2.4a) [29]. This is the equilibrium arrangement if one of the polymer blocks exhibits a volume fraction of $33-37 \%$ [30]. When extending 
from an diblock copolymer, with segments $\mathrm{A}$ and $\mathrm{B}$, to a three component system, with blocks $\mathrm{A}, \mathrm{B}$ and $\mathrm{C}$, the compositional window for the symmetric double gyroid morphology is significantly larger in a ternary phase diagram where the blocks arrange in an ABCBA core-shell structure (Figure $2.4 \mathrm{~b}$, space group: $l a \overline{3} d\left(\mathrm{Q}^{230}\right)$ ). Additionally, a three component system allows access to a chiral phase morphology (Figure 2.4c, space group: $l l 4_{1} 32\left(\mathrm{Q}^{214}\right)$ ) [31, 32].

In fact, linear dichroism and gyrotropic light propagation has been experimentally shown for a chiral alternating gyroid structure made of gold, analogue to Figure 2.4c [33]. Theoretical models predict a negative refractive index in the visible and near-infrared range for a symmetric double gyroid morphology after infiltration with silver or aluminium [28].

In lossless dielectric films, the effect of morphology on the optical response is much less pronounced. For model systems with spherical pores in a symmetric arrangement as illustrated in Figure 2.5a, the pore size and spatial periodicity (SC, BCC, FCC) have no effect on the resulting optical properties. These are simply determined by the pore volume for pore diameters $d_{\text {iam }} \gg \lambda$ and sufficiently thick films $h_{\text {film }} / d_{\text {iam }} \geq 150$ [34]. Nevertheless, the spatial arrangement of material will have an influence on how porosity can be controlled and adjusted. The

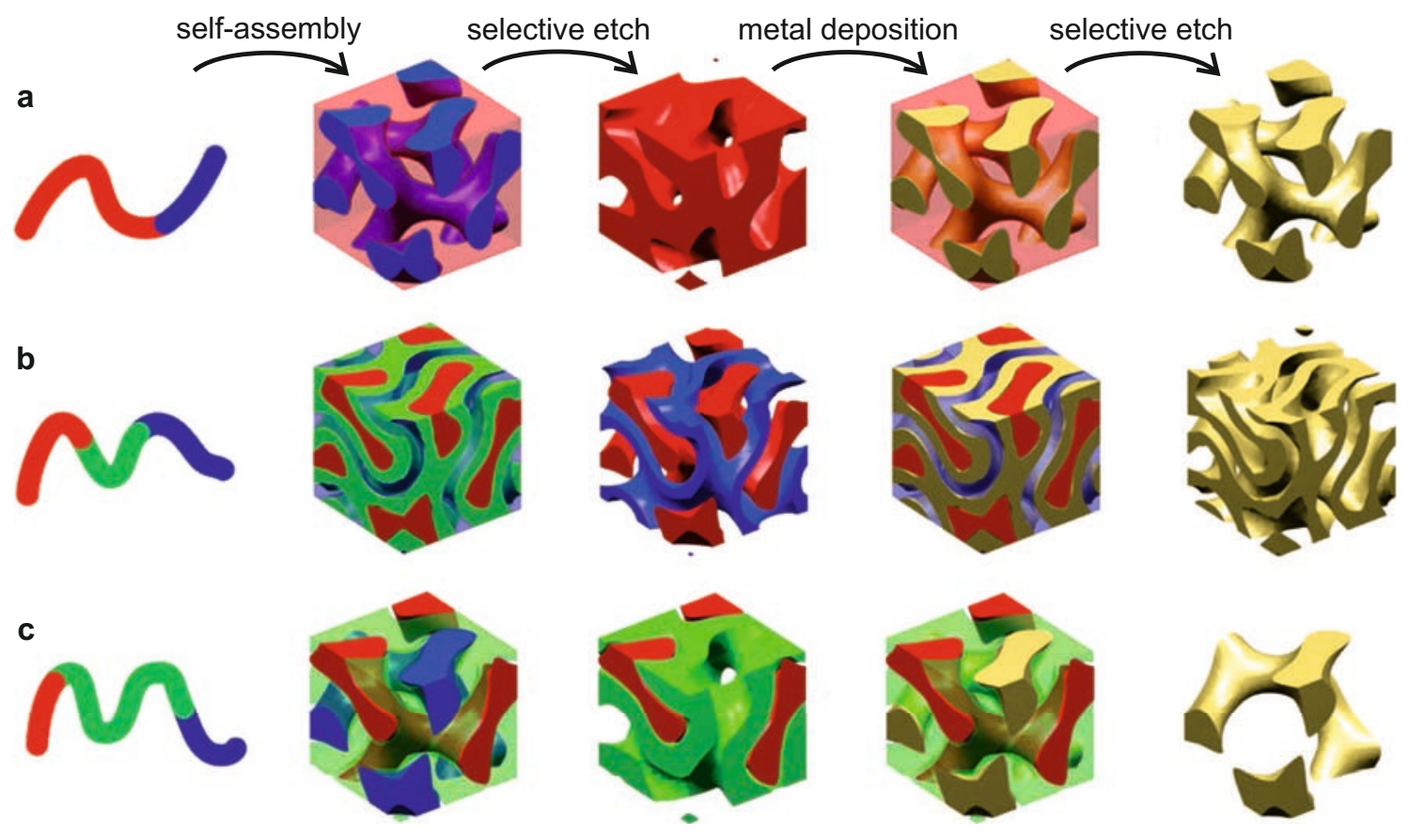

Figure 2.4: BCP route to metamaterials at visible frequencies. The templating approach involves four processing steps: (1) BCP self-assembly in a thin film, (2) selective etching of one of the polymer blocks, (3) backfilling of the template with a metal and (4) selective removal of the residual polymer components. The resulting network symmetry depends on the employed BCP: a) Diblock copolymer yielding a double gyroid morphology. b) Triblock terpolymer resulting in a double gyroid morphology. c) Triblock terpolymer route to an alternating gyroid, a chiral morphology. a) - c) Adapted with permission from [28]. Copyright 2011 Wiley. 
conventional sintering of nanoparticles to form mesoporous films only allows a variation of porosity between $\approx 30 \%-40 \%$ [35]. This is due to the fact that porosity is generated by the random close packing of nanoparticles, which somewhat limits the control over pore size and pore volume [36]. By blending with additional sacrificial material, porosity can be increased up to $60 \%$ [37]. In contrast, the use of BCPs as templates or structure directing agents for the assembly of inorganic films offers a much higher control over pore size and porosity, even if the film morphology may not be of particular importance. This aspect is further explored in Chapter 5.

Mesoporous materials exhibit pores of 5-50 nm length scale, too small to be resolved using visible light. As a consequence, the compositional arrangement can be treated as an effective medium, with optical constants that depend on the volume fractions of the constituents. There are a number of different physical models that relate the macroscopic properties of a medium to the relative volume fraction of its components. The most intuitive approach has been established by Birchak et al., which describes the effective index of the medium by the linear combination of its volume fractions, i.e.

$$
n_{\mathrm{eff}}=\left(1-f_{\mathrm{p}}\right) n_{\mathrm{m}}+f_{\mathrm{p}} n_{\mathrm{p}}
$$

where $f_{\mathrm{p}}$ is the volume fraction of the pores, and $n_{\mathrm{m}}$ and $n_{\mathrm{p}}$ are the refractive indices of the matrix and pore forming material, respectively [38].

A physical model for the relation between the dielectric constant of an lossless optical medium and the polarisability of its constituent molecules has been introduced by Mossotti and Clausius [39, 40].

$$
\frac{n_{\mathrm{eff}}^{2}-1}{n_{\mathrm{eff}}^{2}+2}=\frac{4 \pi}{3} \alpha q,
$$

where $n_{\text {eff }}$ is the effective refractive index of the overall medium, $\alpha$ is the molecular polarisability and $q$ is the number of molecules per unit volume. Their analytic solution is based on a dielectric medium which is built up of polarisable objects in empty space in a perfectly mixed, homogeneous and isotropic configuration [41]. When $\alpha$ is modified to account for the local field of nearby atoms, the Clausius-Mossotti model is also valid for homogeneous solid state materials [42].

The extension of the Clausius-Mossotti equation to a mixture of constituents and volume fractions was developed by L. Lorenz and H.A. Lorenz [43, 44].

$$
\frac{n_{\mathrm{eff}}^{2}-1}{n_{\mathrm{eff}}^{2}+2}=f_{1}\left(\frac{n_{1}^{2}-1}{n_{1}^{2}+2}\right)+f_{2}\left(\frac{n_{2}^{2}-1}{n_{2}^{2}+2}\right),
$$


where $n_{1}$ and $n_{2}$ are the refractive indices of component 1 and 2 and $f_{1}$ and $f_{2}$ are their volume fractions [45]. The Clausius-Mossotti-Lorenz-Lorenz model assumes mixing of polarisable objects on the molecular level. It does not take into account that the constituents may form domains that are large enough to exhibit their own dielectric identity.

The Maxwell-Garnett model considers mesoscopic domains by shifting from a vacuum lattice with polarisable point objects to a host-guest approach for the dielectric components. The minority component $\left(n_{\mathrm{p}}, f_{\mathrm{p}}\right)$ then forms inclusions, i.e. pores, in a host matrix that is formed by the majority component $\left(n_{\mathrm{m}}\right)$.

$$
\frac{n_{\mathrm{eff}}^{2}-n_{\mathrm{m}}^{2}}{n_{\mathrm{eff}}^{2}+2 n_{\mathrm{m}}^{2}}=f_{\mathrm{p}} \frac{n_{\mathrm{p}}^{2}-n_{\mathrm{m}}^{2}}{n_{\mathrm{p}}^{2}+2 n_{\mathrm{m}}^{2}} .
$$

The host-guest model assumes $f_{\mathrm{m}}>>f_{\mathrm{p}}$ and $n_{\mathrm{m}}>n_{\mathrm{p}}$. In cases where the volume fraction of pores becomes comparable or predominant, this approach may not be valid. A more general expression is

$$
\frac{n_{\mathrm{eff}}^{2}-n_{\mathrm{h}}^{2}}{n_{\mathrm{eff}}^{2}+2 n_{\mathrm{h}}^{2}}=f_{\mathrm{a}} \frac{n_{\mathrm{a}}^{2}-n_{\mathrm{h}}^{2}}{n_{\mathrm{a}}^{2}+2 n_{\mathrm{h}}^{2}}+f_{\mathrm{b}} \frac{n_{\mathrm{b}}^{2}-n_{\mathrm{h}}^{2}}{n_{\mathrm{b}}^{2}+2 n_{\mathrm{h}}^{2}},
$$

where $n_{\mathrm{h}}^{2}, n_{\mathrm{a}}^{2}$ and $n_{\mathrm{b}}^{2}$ are the refractive indices of the host and components a and $\mathrm{b}$, respectively. This is equivalent to the Clausius-Mossotti-Lorenz-Lorenz equation for $n_{\mathrm{h}}^{2}=1$. The expression leads to the Maxwell-Garnett model for $n_{\mathrm{h}}^{2}=n_{\mathrm{eff}}^{2}$. For a self-consistent approach with $n_{\mathrm{h}}^{2}=n_{\mathrm{eff}}^{2}$ equation 2.40 reduces to

$$
\left(1-f_{\mathrm{b}}\right) \frac{n_{\mathrm{a}}^{2}-n_{\mathrm{eff}}^{2}}{n_{\mathrm{a}}^{2}+2 n_{\mathrm{eff}}^{2}}+f_{\mathrm{b}} \frac{n_{\mathrm{b}}^{2}-n_{\mathrm{eff}}^{2}}{n_{\mathrm{b}}^{2}+2 n_{\mathrm{eff}}^{2}}=0 .
$$

This is the so-called Bruggeman effective medium approximation. The predictions of the four models are exemplified in Figure $2.5 \mathrm{~b}$ for an porous material with $n_{\mathrm{m}}=2$ in air $\left(n_{\mathrm{p}}=1\right)$. For $f_{\mathrm{p} \text {, air }}=0.3$ the deviation between Bruggeman $\left(n_{\mathrm{eff}}=1.69\right)$, Birchak $\left(n_{\mathrm{eff}}=1.70\right)$ and Maxwell-Garnett $\left(n_{\mathrm{eff}}=1.71\right)$ is well within the experimental uncertainty. In contrast, the deviation becomes significant for highly porous materials. 

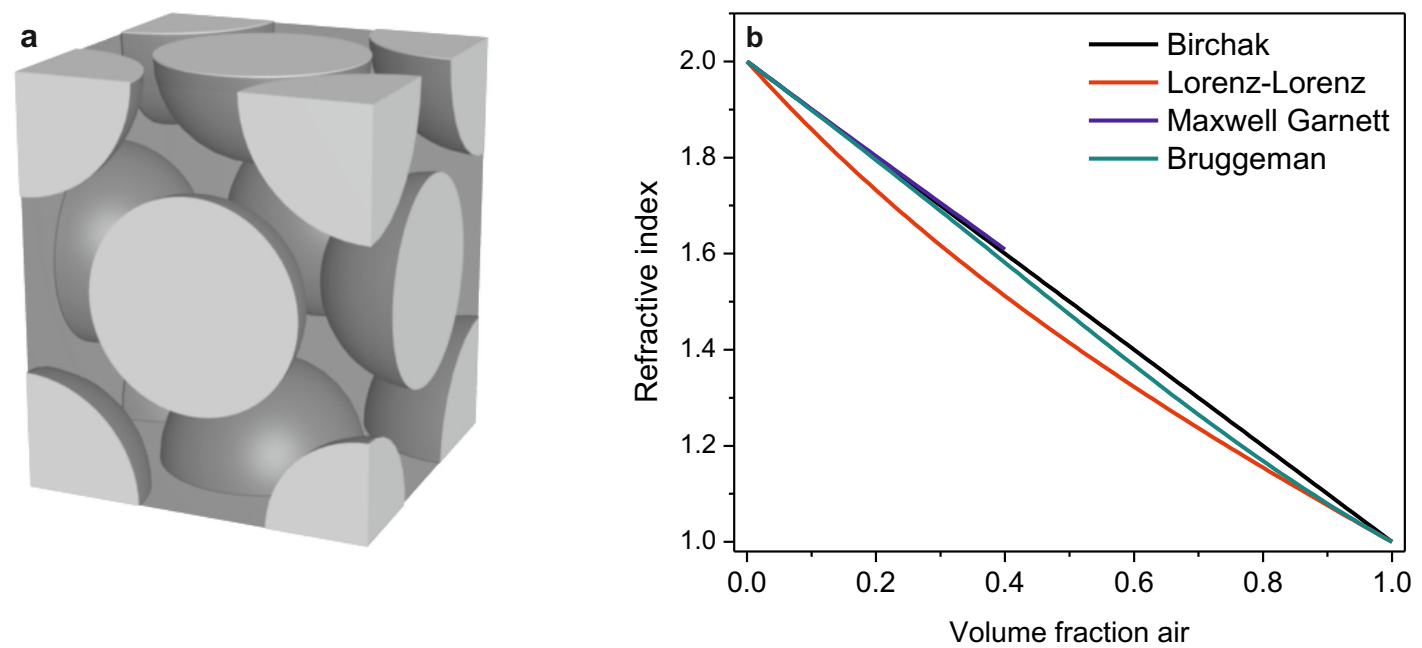

Figure 2.5: Effective medium approximation. a) Schematic of a two component film with closepacked pores. b) Refractive index vs. porosity for different effective medium approximations with $n_{\text {high }}=2$. The Maxwell-Garnett model assumes one majority component and $n_{\mathrm{m}}>n_{\mathrm{p}}$, which is only valid in a limited range. a) Adapted with permission from [46]. Copyright Mathias Kolle.

\section{Bibliography}

[1] J. Maxwell, "A dynamical theory of the electromagnetic field," Philosophical Transactions of the Royal Society of London, vol. 155, pp. 459-512, 1865.

[2] D. Griffiths, Introduction to electrodynamics. Benjamin Cummings, 3rd ed., 1999.

[3] E. Hecht, Optics. Pearson, 4th ed., 2003.

[4] O. Heavens, The optical properties of thin solid films. Dover, 1992.

[5] Wikimedia - public domain graphic http://commons.wikimedia.org, 2011.

[6] P. Rouard, "Etudes des proprietes optiques des lames metalliques tres minces," Annales de Physique, vol. 7, pp. 291-384, 1937.

[7] G. Fowles, Introduction to modern optics. Dover Publications Inc., 1st ed., 1990.

[8] M. Born and E. Wolf, Principles of optics. Cambridge University Press, 7th ed., 1999.

[9] E. Schubert, Light-emitting diodes. Cambridge University Press, 2nd ed., 2006.

[10] M. Kolle, Photonic structures inspired by nature. PhD thesis, University of Cambridge, Physics Department, 2010.

[11] G. Burkhard, E. Hoke, and M. McGehee, "Accounting for interference, scattering, and electrode absorption to make accurate internal quantum efficiency measurements in organic and other thin solar cells," Advanced Materials, vol. 22, no. 30, pp. 3293-3297, 2010.

[12] H. Macleod, Thin film optical filters. Institute of Physics Publishing, 3rd ed., 2001. 
[13] E. Yablonovitch, "Inhibited spontaneous emission in solid-state physics and electronics," Physical Review Letters, vol. 58, no. 20, pp. 2059-2062, 1987.

[14] S. John, "Strong localization of photons in certain disordered dielectric superlattices," Physical Review Letters, vol. 58, no. 23, pp. 2486-2489, 1987.

[15] C. Lopez, "Materials aspects of photonic crystals," Advanced Materials, vol. 15, no. 20, pp. 1679-1704, 2003.

[16] K. Arpin, A. Mihi, H. Johnson, A. Baca, J. Rogers, J. Lewis, and P. Braun, "Multidimensional architectures for functional optical devices," Advanced Materials, vol. 22, no. 10, SI, pp. 1084-1101, 2010.

[17] J. Joannopoulos, S. Johnson, J. Winn, and M. R.D., Photonic crystals: molding the flow of light. Princeton University Press, 2nd ed., 2008.

[18] A. Taflove, Computational electrodynamics, the Finite-Difference Time-Domain Method. Artech House Publishers, 3rd ed., 2005.

[19] K. Ho, C. Chan, and C. Soukoulis, "Existence of a photonic gap in periodic dielectric structures," Physical Review Letters, vol. 65, no. 25, pp. 3152-3155, 1990.

[20] V. Veselago, "Electrodynamics of substances with simultaneously negative electrical and magnetic permeabilities," Soviet Physics Uspekhi-USSR, vol. 10, no. 4, pp. 507-514, 1968.

[21] J. Pendry, "Negative refraction makes a perfect lens," Physical Review Letters, vol. 85, no. 18, pp. 3966-9, 2000.

[22] R. Shelby, D. Smith, and S. Schultz, "Experimental verification of a negative index of refraction," Science, vol. 292, no. 5514, pp. 77-79, 2001.

[23] V. Shalaev, W. Cai, U. Chettiar, H. Yuan, A. Sarychev, V. Drachev, and A. Kildishev, "Negative index of refraction in optical metamaterials," Optics Letters, vol. 30, no. 24, pp. 3356-3358, 2005.

[24] G. Dolling, M. Wegener, C. Soukoulis, and S. Linden, "Negative-index metamaterial at 780 nm wavelength," Optics Letters, vol. 32, no. 1, pp. 53-55, 2007.

[25] S. Burgos, R. de Waele, A. Polman, and H. Atwater, "A single-layer wide-angle negativeindex metamaterial at visible frequencies," Nature Materials, vol. 9, no. 5, pp. 407-412, 2010.

[26] J. Pendry, “A chiral route to negative refraction," Science, vol. 306, no. 5700, pp. 13531355, 2004.

[27] S. Zhang, Y.-S. Park, J. Li, X. Lu, W. Zhang, and X. Zhang, "Negative refractive index in chiral metamaterials," Physical Review Letters, vol. 102, no. 2, 2009.

[28] K. Hur, Y. Francescato, V. Giannini, S. Maier, R. Hennig, and U. Wiesner, "Threedimensionally isotropic negative refractive index materials from block copolymer selfassembled chiral gyroid networks," Angewandte Chemie - Int. Ed., vol. 123, no. 50, pp. 12191-12195, 2011. 
[29] D. Haduk, P. Harper, S. Gruner, C. Honeker, G. Kim, E. Thomas, and L. Fetters, "The gyroid - a new equilibrium morphology in weakly segregated diblock copolymers," Macromolecules, vol. 27, no. 15, pp. 4063-4075, 1994.

[30] C. Park, J. Yoon, and E. Thomas, "Enabling nanotechnology with self assembled block copolymer patterns," Polymer, vol. 44, no. 22, pp. 6725-6760, 2003.

[31] T. Epps, E. Cochran, T. Bailey, R. Waletzko, C. Hardy, and F. Bates, "Ordered network phases in linear poly (isoprene-b-styrene-b-ethylene oxide) triblock copolymers," Macromolecules, vol. 37, pp. 8325-8341, 2004.

[32] Y. Mogi, M. Nomura, H. Kotsuji, K. Ohnishi, Y. Matsushita, and I. Noda, "Superlattice structures in morphologies of the ABC triblock copolymers," Macromolecules, vol. 27, no. 23, pp. 6755-6760, 1994.

[33] S. Vignolini, N. A. Yufa, P. S. Cunha, S. Guldin, I. Rushkin, M. Stefik, K. Hur, U. Wiesner, J. J. Baumberg, and U. Steiner, "A 3D optical metamaterial made by self-assembly," Advanced Materials, vol. 24, no. 10, 2012.

[34] N. Hutchinson, T. Coquil, A. Navid, and L. Pilon, "Effective optical properties of highly ordered mesoporous thin films," Thin Solid Films, vol. 518, no. 8, pp. 2141-2146, 2010.

[35] M. Calvo, S. Colodrero, N. Hidalgo, G. Lozano, C. Lopez-Lopez, O. Sanchez-Sobrado, and H. Míguez, "Porous one dimensional photonic crystals: novel multifunctional materials for environmental and energy applications," Energy $\mathcal{F}$ Environmental Science, vol. 4, pp. 4800-4812, 2011.

[36] S. Burnside, V. Shklover, C. Barbé, P. Comte, F. Arendse, K. Brooks, and M. Grätzel, "Self-organization of $\mathrm{TiO}_{2}$ nanoparticles in thin films," Chemistry Of Materials, vol. 10, no. 9, pp. 2419-2425, 1998.

[37] C. Lopez-Lopez, S. Colodrero, S. Raga, H. Lindstrom, F. Fabregat-Santiago, J. Bisquert, and H. Míguez, "Enhanced diffusion through porous nanoparticle optical multilayers," Journal Of Materials Chemistry, vol. 22, no. 5, pp. 1751-1757, 2012.

[38] J. Birchak, C. Gardner, J. Hipp, and J. Victor, "High dielectric-constant microwave probes for sensing soil-moisture," Proceedings Of The IEEE, vol. 62, no. 1, pp. 93-98, 1974.

[39] O. Mossotti, Memorie Di Matematica E Di Fisica Della Societa Italiana Delle Scienze Residente In Modena. Nella Tipografia Camerale Modena, 1850.

[40] R. Clausius, Die mechanische Behandlung der Elektrizität. 1879.

[41] P. Van Rysselberghe, "Remarks concerning the Clausius-Mossotti law," Journal Of Physical Chemistry, vol. 36, no. 4, pp. 1152-1155, 1932.

[42] R. Feynman, R. Leighton, and M. Sands, Feynman Lectures on Physics. Vol. 2, chap. 32. Addison Wesley, 1989.

[43] L. Lorenz, "Über die Refractionsconstante," Annalen der Physik, vol. 11, pp. 70-103, 1880.

[44] H. Lorenz, "Über die Beziehung zwischen der Fortpflanzungsgeschwindigkeit des Lichtes der Körperdichte," Annalen der Physik, vol. 9, pp. 641-665, 1880. 
[45] D. Aspnes, "Optical-properties of thin-films," Thin Solid Films, vol. 89, no. 3, pp. 249262, 1982.

[46] M. Kolle, personal graphics collection 


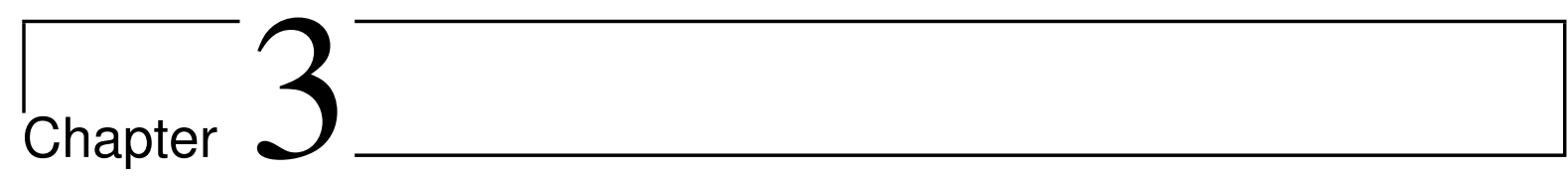

\section{Structure-function interplay in dye-sensitised solar cells}

The design, fabrication and characterisation of novel electrode architecture for dye-sensitised solar cells was of central importance for this thesis. The following chapter aims to explain the fundamental principles of this photovoltaic concept, elucidate the interplay between the electrode architecture and device function and highlight possible ways to increase the power conversion efficiency.

Absorption of light followed by the generation and transport of charge carriers to the electrodes are the principal functions of any photovoltaic cell. In conventional p-n junction-based photovoltaic devices these tasks are carried out by the same material, an inorganic semiconductor. The working principle of dye-sensitised solar cells is fundamentally different and takes inspiration from photosynthesis [1]. Light absorption and charge generation occur separately in specifically designed device components. The light harvesting complex is a photoactive dye molecule which is anchored to the surface of a wide band-gap semiconductor and surrounded by a redox medium. Upon absorption of light, incident photons stimulate the dye molecule to form an excitonic state.

With appropriate energy-level alignment of the device components, charge separation occurs at the interface to the electron and hole conducting materials. Electrons are injected into the conduction band of an inorganic semiconductor and transported to the electrode. Regeneration of the oxidised dye takes place via an electron-donor species, typically an iodine $\left(3 \mathrm{I}^{-} / \mathrm{I}_{3}^{-}\right)$-based liquid electrolyte. Initial work dates back as early as 1887 when James Moser showed the extension of the absorption spectrum of a photoelectrochemical cell by the sensitisation with a light-absorbing dye molecule [2]. Yet, the acceptance of DSCs as a promising photovoltaic concept only arose from the seminal work of O'Regan and Grätzel who introduced a novel electrode architecture in the form of a mesoporous $\mathrm{TiO}_{2}$ film with 780 -fold 
surface area. The associated substantial increase in dye-loading led to an order of magnitude rise in the conversion efficiency of incident photons to electrons [3]. The strategy of sintering nanometre-sized $\mathrm{TiO}_{2}$ particles to form an electron conducting random network of extremely large surface area is still widely used today and a central part of the conventional device architecture, shown in Figure 3.1.

Like any single-junction photovoltaic cell, conventional dye-sensitised solar cells obey the Shockley-Queisser limit for the power conversion of the sun's black body radiation, shown in Figure 3.1b. Accordingly, the ideal excitation energy $\Delta E$ should be $\sim 1.1 \mathrm{eV}(\lambda \geq 1125 \mathrm{~nm})$, which would result in a power conversion efficiency of $33.7 \%$, if the dye's excitation energy could be fully converted into electrical energy [4]. These values are far from reach in any experimental realisation. Historically, the ruthenium-based photosensitisers N3 (1991, [5]), N719 (1999, [6]) and N749 (2001, [7]) were milestones in dye synthesis and enabled power conversion efficiency records of $10.0 \%$ [5], $10.6 \%$ [8] and $11.1 \%$ [9], respectively. Recently, a shift from the iodine to a cobalt-based electrolyte in combination with the development of an organic donor- $\pi$-acceptor porphyrin dye enabled to increase the current benchmark to $12.3 \%$ [10].

A typical current-voltage curve of a DSC under illumination with simulated sunlight is shown in Figure 3.1c. The performance is typically normalised to the area of the illumination and given as a density, such as the current density $J\left[\mathrm{~mA} / \mathrm{cm}^{2}\right]$ or the power density $P$ $\left[\mathrm{mW} / \mathrm{cm}^{2}\right]$. The current output follows a classical diode characteristics with an offset due to the generated photocurrent, here plotted with a positive sign [11]. Forward bias signifies applying a negative potential to the n-type working electrode. The operating regime of a solar cell thus lies in forward bias between $0 \mathrm{~V}$ and the open circuit potential $V_{\mathrm{oc}}$, where the overall cell current is zero. The maximum photocurrent generated at $0 \mathrm{~V}$ is defined as as short circuit current $\left(J_{\mathrm{sc}}\right)$. The corresponding generated power density of the device $(P=J \cdot V)$ peaks at the maximum power point $\left(P_{\max }\right)$, which determines the optimum forward bias $\left(V_{\max }\right)$ and the related current density $\left(J_{\max }\right)$. The fill factor $(F F)$ quantifies the deviation of the current-voltage characteristics from a square curve and is therefore a key quality factor of the device, with

$$
F F=\frac{J_{\max } V_{\max }}{J_{\mathrm{sc}} V_{\mathrm{oc}}}=\frac{P_{\max }}{J_{\mathrm{sc}} V_{\mathrm{oc}}} .
$$

The power conversion efficiency $\eta$ is the ratio of generated power density to incident illumination irradiance:

$$
\eta=\frac{P_{\max }}{P_{\text {in }}}=\frac{J_{\mathrm{sc}} V_{\mathrm{oc}} F F}{P_{\text {in }}} .
$$

The current-voltage curve shown in Figure 3.1c corresponds to an efficiency $\eta$ of $10.6 \%$ and a fill factor $F F$ of 0.75 . Another fundamental device parameter is the so-called incident-photon- 
to-electron conversion efficiency (IPCE), which correlates the number of generated electrons, measured as photocurrent at $0 \mathrm{~V}$, to the incident photon flux at a particular wavelength.

$$
\operatorname{IPCE}=\frac{n_{\text {electrons }}(\lambda)}{n_{\text {photons }}(\lambda)}=\frac{J_{\text {sc }}}{P_{\text {in }}} \frac{\text { hc }}{\mathrm{e} \lambda}
$$

A characteristic plot of $\operatorname{IPCE}(\lambda)$ for conventional devices employing the sensitisers $\mathrm{N} 719$ and N749 is shown in Figure 3.1d.
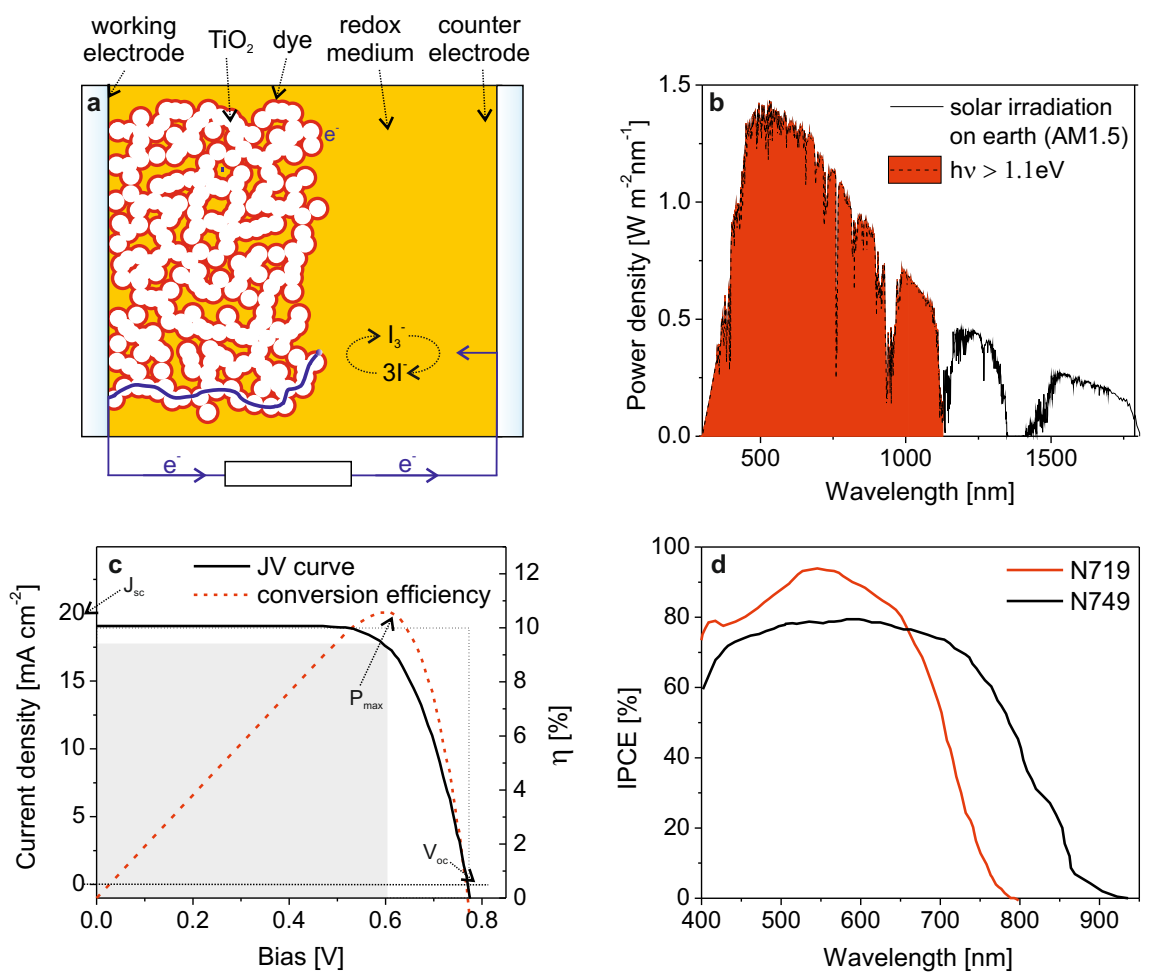

Figure 3.1: Key device characteristics of dye-sensitised solar cells a) Schematic of a conventional device architecture. Nanometre-sized $\mathrm{TiO}_{2}$ particles form an electron conducting random network with high surface area. A monolayer of light-absorbing dye molecules is chemisorbed onto the $\mathrm{TiO}_{2}$ and surrounded by an electrolyte. Upon photoexcitation of the dye, electrons are injected into the $\mathrm{TiO}_{2}$, while the oxidised dye is regenerated by the electron-donating redox medium, which is subsequently reduced at the counter electrode. b) Power density of solar irradiation on earth as a function of photon wavelength (AM 1.5, reference [12]). The spectral window for an ideal single junction solar cell $(\Delta E=1.1 \mathrm{eV})$ is shown as a dashed line. c) Current-voltage $(J-V)$ characteristics of a conventional DSC under illumination (dye N719). The generated power density of the device $(P=J \cdot V)$ is shown in red. The device performance can be described by the key parameters $J_{\mathrm{sc}}$ (short circuit current), $V_{\mathrm{oc}}$ (open circuit potential) and $P_{\max }$ (maximum power point). d) Incident-photon-to-electron conversion efficiency (IPCE) as a function of wavelength for devices sensitised with the dye $\mathrm{N} 719$ (red) and N749 (black), respectively. c) data reprinted with permission from [8]. Copyright 2003 Elsevier. d) N749 data reprinted with permission from [7]. Copyright 2001 American Chemical Society. N719 data reprinted with permission from [13]. Copyright 2007 Elsevier. 
The functioning of a DSC is largely determined by the energy level alignment between the individual components as well as the underlying kinetics of the charge separation and charge transfer processes. Figure 3.2 represents an energy level diagram of a conventional liquid electrolyte DSC with the underlying reaction kinetics. The fundamental processes include:

(1) Photo-excitation of the dye [14].

(2) Electron injection into the metal oxide $[15,16]$.

(3) Electron transport to the working electrode [17].

(4) Regeneration of the oxidised dye by electron transfer from donor species $[18,19]$.

(5) Hole transport to the electrode [20].

(6) Reduction of the oxidised donor [21].

and the competing charge recombination processes:

(7) Excited state decay of the dye [14].

(8) Regeneration of the oxidised dye by back transfer of into $\mathrm{TiO}_{2}$ injected electrons [22].

(9) Recombination of injected electron with acceptor species in the redox medium [23, 24].

Dye-sensitised solar cells are a promising photovoltaic concept for a number of reasons. The spatial separation of light absorption and charge generation makes the devices very stable against photocorrosion. This is due to the band gap of the employed semiconductor material in the photoanode, being a measure of the chemical bond strength. Metal oxides like $\mathrm{TiO}_{2}$ or $\mathrm{Nb}_{2} \mathrm{O}_{3}$ have an absorption edge towards the ultraviolet and are therefore stable against photodegradation in the spectral range of highest photon energy flux, i.e. the visible spectrum [26]. Unlike conventional p-n junction solar cells, where excitons are generated in the bulk and then need to diffuse to the p-n interface in order to be separated and extracted, exciton generation in DSCs takes place directly at the material's interface. As a consequence the demand on materials purity is much lower, which implies that processing under vacuum, ultra high temperature or cleanroom classification is generally not necessary [27]. Recent advances in the synthesis of organic dyes have eliminated the need for the rare ruthenium-based sensitisers, meaning that all device components are now abundant and promise significantly lower processing costs than existing technologies $[10,28]$. The variety of colours and transparencies distinguishes DSCs from other photovoltaic concepts and makes them ideally suited for integrated architecture and building design $[29,30]$. 


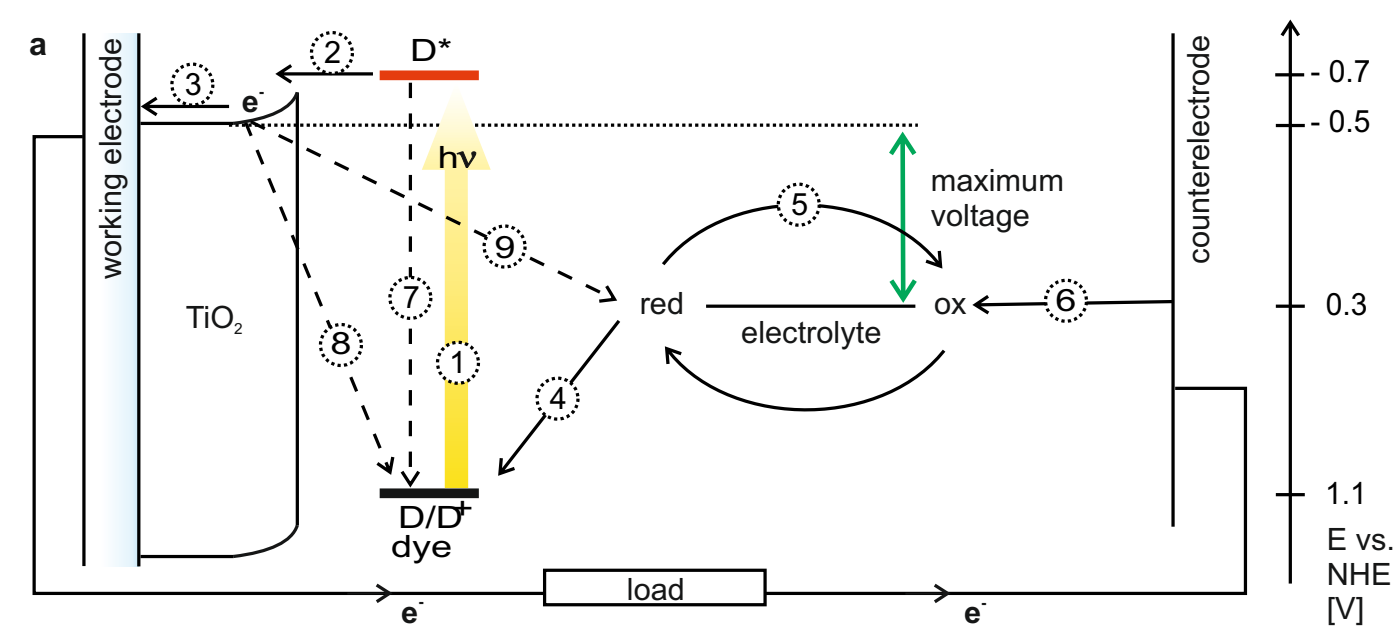

b

(2) charge injection into $\mathrm{TiO}_{2}$

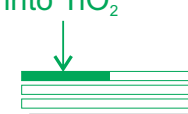
$10^{-13}$ $0^{-13}$ ps

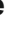

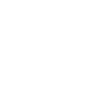


1. Loss in potential: The established high performance black dye N749 has an absorption onset at $\sim 920 \mathrm{~nm}$, corresponding to an optical bandgap of around $1.35 \mathrm{eV}$. However, devices only deliver $V_{\mathrm{oc}}=0.74 \mathrm{~V}$ (in the case of N719: $E_{\text {gap }}=1.48 \mathrm{eV} ; V_{\mathrm{oc}}=0.80 \mathrm{~V}$ ). As schematically illustrated in Figure 3.2a, photoelectrochemical cells rely on the progression of charge carrier generation followed by several charge carrier transfer processes. In order to make these energetically more favourable than parasitical recombination processes, a certain loss in potential is required. The dye regeneration via the redox reaction of iodine $\left(3 \mathrm{I}^{-} / \mathrm{I}_{3}^{-}\right)$consumes $\sim 0.6 \mathrm{~V}$, due to the fact that the reaction is a two-step process $[32,33]$. The oxidation of the electrolyte $\left(2 \mathrm{I}^{-} \rightarrow \mathrm{I}_{2}^{-}\right)$requires an overpotential of $0.2-0.3 \mathrm{eV}$, followed by the dismutation of $2 \mathrm{I}_{2}^{-} \rightarrow \mathrm{I}_{3}^{-}+\mathrm{I}^{-}$, with a further demand of $\sim 0.3 \mathrm{eV}$. Alternative routes that employ a one-electron regeneration process may only require an overall loss in potential of $0.4 \mathrm{eV}$ instead of up to $0.75 \mathrm{eV}$, which would make power conversion efficiency of $20 \%$ viable [31]. Yet, changing from a two-electron to a one-electron regeneration process has far-reaching consequences on the kinetic balance of the system as recombination of injected electrons with oxidised acceptor species becomes more likely.

2. Loss in absorption/photocurrent: As shown in Figure 3.1d, the IPCE can reach values up to $80-90 \%$ in high efficiency devices but only in a limited spectral range. The IPCE then drops drastically in regions of poor dye extinction, i.e. in the tail of the absorption spectra of the dye [34]. The development of dyes that are more panchromatic and of higher exctinction coefficient is therefore essential [35]. Another effective parameter is the photonic design of the photoanode architecture. The addition of a layer of scattering particles is widely used to increase the IPCE, particularly in the red/infrared region of the spectrum due to the greatly enhanced photon path length $[9,34]$. Further control of the light-matter interaction can be achieved by integration of one-dimensional (1D) or three-dimensional (3D) photonic crystals [36, 37].

3. Loss in fill factor: The origin for the deviation of the $J-V$ curve from an ideal diode characteristic are numerous. Ohmic resistance arises from the diffusion impedance of the electrolyte $\left(0.7-2 \Omega / \mathrm{cm}^{2},[20]\right)$, the charge transfer resistance of platinum-based counterelectrode $<1 \Omega / \mathrm{cm}^{2}$ and the sheet resistance of the transparent conducting electrode (TCE) [19]. This will not affect the open circuit voltage but seriously deteriorates the diode characteristics, as the overall fall of potential is now divided between the diode and an ohmic resistor, which are connected in series [32]. Another impairing influence for the fill factor arises from the recombination of electrons in the substrate with vacancies in the donor species, particular for alternative redox couples with one-electron 
transfer. This parallel (or shunt) resistance can somewhat be suppressed by coating the TCE with a compact metal oxide layer [38]. Furthermore, the extraction efficiency of injected electrons is voltage-dependent and near-unity IPCE values measured at $\mathbf{J}_{\mathrm{sc}}$ may not necessarily lead to similarly efficient extraction under working conditions. At the maximum power point, the electron density in the $\mathrm{TiO}_{2}$ is typically doubled compared to short circuit conditions, leading to an increase in the recombination rate [39]. Poorly working devices with an extremely low $F F$ are often the consequence of an s-shaped $J-V$-curve under illumination. This may be caused by photo-injected electrons that cannot escape the metal oxide network due to insufficient percolation paths to the TCE or a degraded counter electrode [39].

The focus of this dissertation was to employ the structure control offered by soft matter self-assembly to explore the structure-function interplay of DSC photoanodes. Two different strategies were followed:

1. Increase in photocurrent, photovoltage and fill factor by the design of novel electrode structures with improved charge transport properties. See Section 3.1.

2. Increase IPCE in regions of low dye absorption by enhancing light-matter interaction through the integration of photonic crystal elements. See Section 3.2 for the design of alternative device architectures with photonic properties.

\subsection{Novel electrode structures for enhanced charge carrier transport}

Several requirements for the morphology of the electron-conducting photoanode are apparent when studying the operating principle of DSCs. As photons are only absorbed at the n-dye-p interface, the photoanode needs to exhibit an extremely large surface to multiply the available area for dye anchoring. Even state-of-the-art dyes with high extinction coefficients require a photosensitive interfacial area that is a hundred- to thousand-fold increased compared to a flat film. Upon excitation and electron injection, the oxidised dye has to be promptly reduced by a surrounding regenerating redox mediator. The pores of the electron-conducting network therefore need to be large enough to allow ion diffusion for a solution-based electrolyte or pore infiltration with a solid state hole conductor. Furthermore the porous network has to offer direct percolation paths for the extraction of charges. Thus, an idealised photoanode morphology should be mesoporous (i.e. exhibit porosity on the $10 \mathrm{~nm}$ length scale), bicontinuous and offer adequate charge carrier transport. 
The efficiency leap in the seminal work of O'Regan and Grätzel in 1991 was mainly due to a novel photoanode structure which sufficiently fulfilled these requirements - a mesoporous network generated by the random sintering of $20 \mathrm{~nm}$-sized $\mathrm{TiO}_{2}$ particles. Although random in pore size and morphological order, this electrode structure is still the gold standard in today's devices and a fundamental component in all record-breaking DSCs [9, 10]. Two main drawbacks arise from the random sintering of nanoparticles to form a mesoporous network, namely poor charge transport [40] and a lack of control over the pore size distribution [41].

The electron mobility typically decreases by several orders of magnitudes from a value of $\mu \sim 10 \mathrm{~cm}^{2} /$ Vs for single crystal anatase [42] to around $\mu \sim 10^{-1}-10^{-5} \mathrm{~cm}^{2} /$ Vs in nanoparticle films [40, 43, 44]. In comparison to a single-crystal model system where only optical phonon scattering and lattice defects limit the mean free path of propagating electrons [45], a number of causes slow down electron diffusion in random networks, as illustrated in Figure 3.3a. Charge traps are located at the grain boundaries between the crystalline nanoparticles as well as at the particles' surfaces and in the bulk. These lead to the formation of an exponentially decreasing tail of sub-band gap states below the conduction band edge [46, 47]. Furthermore electrostatic interactions with surrounding ions in the redox medium influence the electron diffusion and lead to trapping and an ambipolar diffusion process $[46,48]$. It is therefore widely agreed that electron transport in such mesoporous networks is consistent with a multiple trapping model, where generated electrons mostly populate localised states below the conduction band and only diffuse towards the electrode in iterative cycles of thermal detrapping and trapping [39, 46, 47, 49]. This is illustrated in Figure 3.3b.

Accordingly, the effective electron diffusion coefficient $D_{\text {el,eff }}$ in $\mathrm{TiO}_{2}$ can be correlated with the concentration of electrons that are localised in sub-band gap traps $\left(\rho_{\mathrm{el}, \mathrm{t}}\right)$ and delocalised in the conduction band $\left(\rho_{\mathrm{el}, \mathrm{c}}\right)$.

$$
D_{\mathrm{el}, \mathrm{eff}}=\left(1+\frac{\partial \rho_{\mathrm{el}, \mathrm{t}}}{\partial \rho_{\mathrm{el}, \mathrm{c}}}\right)^{-1} D_{\mathrm{el}, 0}
$$

where $D_{\mathrm{el}, 0}$ is the diffusion coefficient of electrons in the conduction band (i.e. in a trap-free system) [47].

While experimental evidence for a multiple trapping-governed electron transport process is convincing $[48,49]$, the exact origin of the multitude of transport-limiting traps is still under debate [25]. In particular the decisive role of traps on the particle surface $[50,51]$ and at the interparticle's grain boundaries [52] has been experimentally shown. Furthermore the geometry plays a decisive role as the random dense packing of nanoparticles leads to a greatly enhanced number of dead-ends, i.e. particles with only one neighbour, for porosities above $50 \%$, resulting in a percolation threshold at $76 \%$ porosity [53]. The development of network 

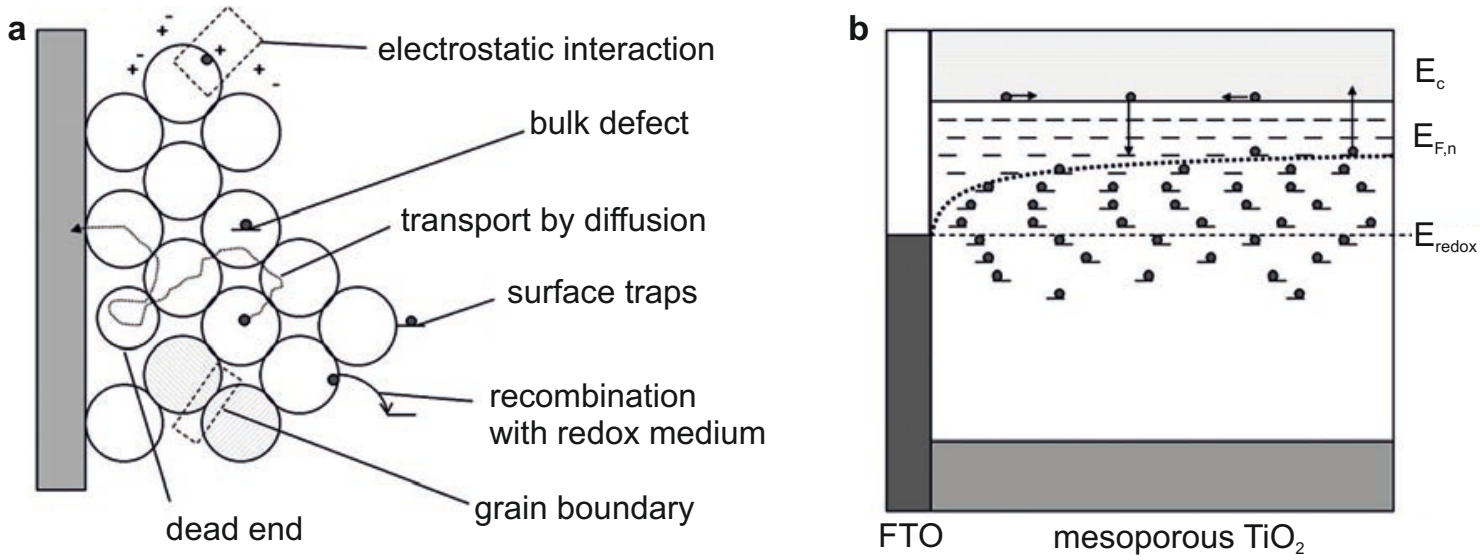

Figure 3.3: Electron transport in mesoporous $\mathbf{T i O}_{2}$. a) Simplified drawing to illustrate electron transport across a random network of nanocrystalline particles. Diffusion of electrons to the working electrode is limited by traps located at the particle grain boundaries, their interfaces and in the bulk of the particles, as well as by dead ends in the percolation path. Furthermore electrostatic interactions with surrounding ions in the redox medium influence the diffusion process, leading to ambipolar diffusion and trapping. b) Schematic diagram of electron transport to the electrode under short-circuit conditions as proposed by a multiple trapping model. More than $90 \%$ of the generated electrons are trapped in localised sub-band gap states below the conduction band edge [39]. Transport in the conduction band only takes place in iterative cycles of thermal detrapping and trapping. a) - b) Adapted with permission from [19]. Copyright 2010 American Chemical Society.

structures with well defined bicontinuous geometries, large and oriented nanocrystals and stoichiometrically annealed surfaces should therefore result in significantly improved electron mobilities.

Historically, the poor electronic properties of a random nanoparticle network were not a major limiting factor due to the slow recombination kinetics for the iodine-based redox couple [33]. Yet, one of the main recent trends is to reduce the loss-in potential and improve device efficiency by moving from a two-electron to a one-electron regeneration process. Examples include the use of an electrolyte with a cobalt-based redox couple [10] or an organic solid state hole transporting material $[54,55]$ with reported $V_{\text {oc }}$ up to $0.97 \mathrm{~V}$ and $0.99 \mathrm{~V}$, respectively. These systems furthermore eliminate the corrosion problems of the iodine-based electrolyte, which limits the lifetimes of the cells [28]. However, due to faster recombination kinetics in a one-electron redox system, the poor electron transport properties of nanoparticle-based films deteriorate photocurrent, fill factor and photovoltage through low charge collection efficiencies, faster interfacial recombination and high internal resistance $[25,56]$. A further drawback of the standard nanoparticle network for new generation DSCs is the lack of control over the pore size distribution [41]. Cobalt complexes are bulky and therefore need well-defined 
percolation paths with sufficiently large pores to avoid mass transport limitations to the counterelectrode $[10,56]$. For solid state DSCs, a heterogeneous pore size distribution may reduce pore filling when infiltrating the viscous materials $[57,58]$. As a consequence both device systems are somewhat limited in the maximum film thickness, which particularly affects the IPCE in the tail of the absorption spectrum [5].

Several concepts for nanostructured photoanodes of next generation DSCs are presented in Figure 3.4. A cross-sectional and close-up view of a standard nanoparticle network is shown in Figure 3.4a,b. Crystalline $\mathrm{TiO}_{2}$ nanorods or nanotubes have attracted much interest as alternative morphology. These should exhibit greatly enhanced charge carrier transport properties as the percolation path is in principal 1D and therefore much more direct [59] (Figure 3.4c-e). Hydrothermal growth allows the formation of $\mu \mathrm{m}$ long single crystal rutile nanorods $[60,61]$. Advances in the electrochemical anodisation of titanium have led to the fabrication of up to $\sim 20 \mu \mathrm{m}$ long $\mathrm{TiO}_{2}$ nanotubes [62]. Highest electron mobilities are expected in systems that exhibit the growth of single-crystalline wires $[61,63]$ or quasi-single crystalline growth through the oriented attachment of crystalline fragments [52, 59, 64]. On the other hand, these 1D arrays suffer from a greatly reduced surface area per unit volume due to the conformal alignment and lack of nanoporosity associated with the roughness of nanometre-sized crystals.

Efforts are therefore drawn towards the fabrication of hierarchical structures that are typically tree-like, i.e. they consist of a highly conductive one-dimensional backbone that branches out into a network of feeding pathways. This can be realised by pulsed laser ablation [65] (Figure 3.4g) or hydrothermal growth [66]. While it seems rational that these nanostructures exhibit greatly improved electronic properties, there is still a lack of experimental evidence for this assumption. Single crystal $\mathrm{TiO}_{2}$ nanorods can only synthesised rutile, which is a somewhat less ideal n-type semiconductor for DSCs. Thus, the electron transport rates found in single crystal rutile nanorods are similar to random anatase nanoparticle networks [67]. Nanotube arrays made from electrochemical anodisation are polycrystalline and show comparable electron transport properties to random nanoparticle networks of similar crystallite size distribution $[68,69]$. This is in contrast to a reported increase in electron transport properties by orders of magnitude when one-dimensional growth is governed by the oriented attachment of nanocrystals to form a random network of fibrils [59].

An alternative approach to control structure formation of the photoanode is to use a sacrificial template. The potential of block copolymers (BCPs), which can self-assemble into highly ordered, bicontinuous arrangements and allow morphology replication by inorganic materials, is discussed in Chapter 1.2. An example of a freestanding $\mathrm{TiO}_{2}$ gyroid network is shown in Figure $3.4 \mathrm{~g}$, which was fabricated by electrochemical deposition of $\mathrm{TiO}_{2}$ into one selectively degraded phase of the BCP film [70]. In contrast to 1D networks, the use of BCPs as sacrificial 
templates or structure-directing agents allows the fine control over the morphology formation in $3 \mathrm{D}$ and therefore a much wider parameter space for probing the structure-function interplay in dye-sensitised solar cells [71].
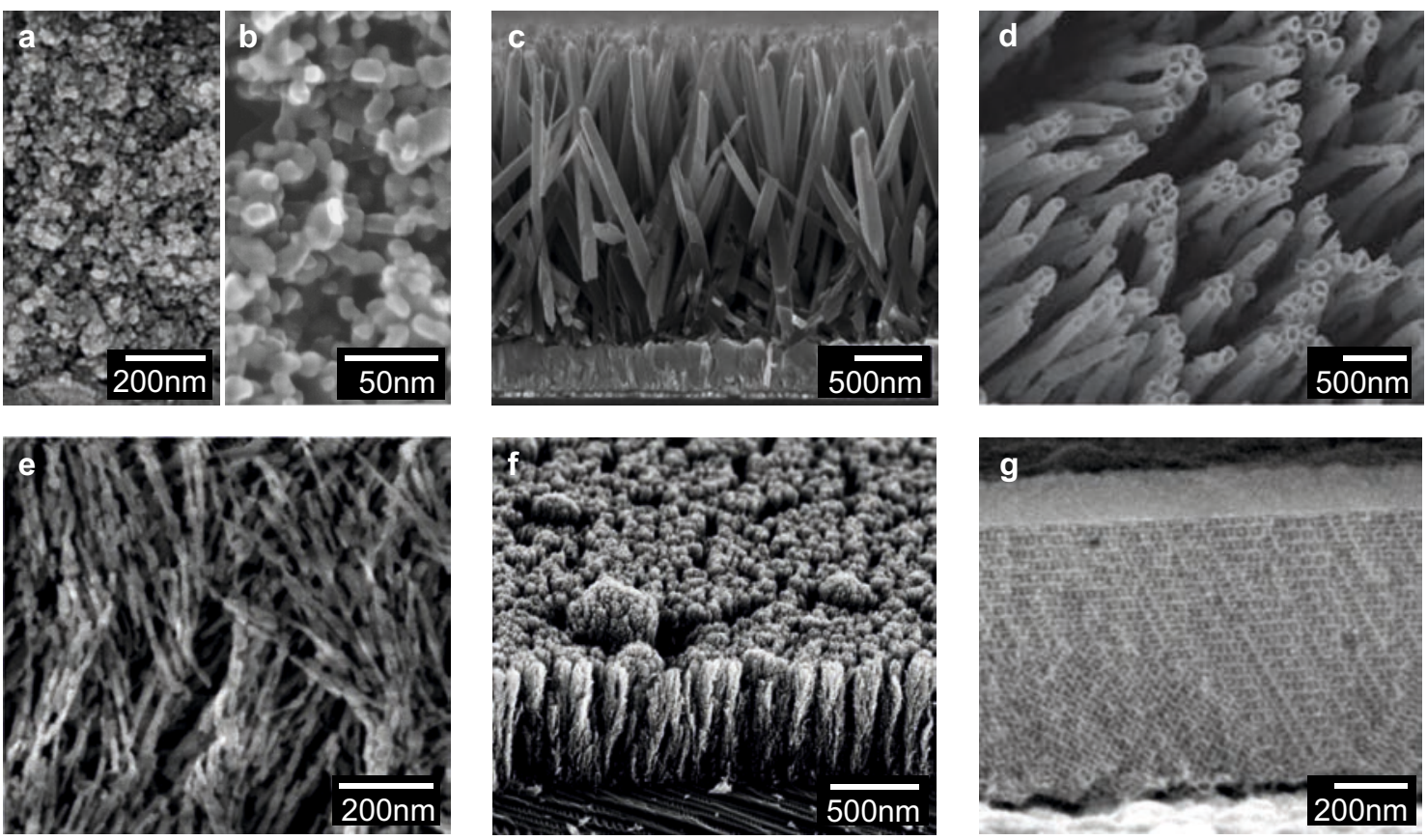

Figure 3.4: Scanning electron microscopy of novel electrode structures for DSCs. a) Crosssectional and b) close-up view of a conventional $\mathrm{TiO}_{2}$ electrode, generated by the random sintering of $\sim 20 \mathrm{~nm}$-sized nanoparticles. c) Cross-sectional image of single-crystalline $\mathrm{TiO}_{2}$ nanorods fabricated by hydrothermal growth in solution. d) Top view of $\sim 20 \mu \mathrm{m}$ long $\mathrm{TiO}_{2}$ nanotubes, grown by electrochemical anodisation of titanium. e) Micrograph of a 3D fibrous network of crystalline $\mathrm{TiO}_{2}$ nanowires synthesised by hydrothermal growth (top view). f) Cross-sectional image of an array of hierarchical, tree-like $\mathrm{TiO}_{2}$ nanostructures that were generated by pulsed lased deposition. g) Cross-sectional view of $\mathrm{TiO}_{2}$ resembling the bicontinuous gyroid morphology generated by electrochemical deposition of $\mathrm{TiO}_{2}$ into a sacrificial block copolymer template. $\boldsymbol{a}$ ) Adapted with permission from [57]. Copyright 2006 Elsevier. b) Adapted with permission from [1]. Copyright 2000 John Wiley $\mathcal{E}$ Sons. c) Adapted with permission from [61]. Copyright 2009 American Chemical Society. d) Adapted by permission from Macmillan Publishers Ltd: Nature Materials [62], copyright 2009. e) Adapted with permission from [59]. Copyright 2010 American Chemical Society. f) Adapted with permission from [65]. Copyright 2010 American Chemical Society. f) Adapted with permission from [70]. Copyright 2009 American Chemical Society. 


\subsection{Photonic device architectures for enhanced light absorp- tion}

Light harvesting in a DSC is fundamentally related to the absorption spectrum of the lightsensitive component. Following the Beer-Lambert law, the absorbance $A$ of light in a photoactive medium is defined as

$$
A(\lambda)=-\log _{10}\left(\frac{I}{I_{0}}\right)=\varepsilon(\lambda) c l,
$$

with $I$ and $I_{0}$ the intensity for incident and transmitted light, $\varepsilon$ the molar extinction coefficient of the dye, $c$ the molar concentration and $l$ the photon path length.

As the film thickness of the photoanode is somewhat restricted due to mass transport limitations and recombination dynamics, the only parameter that can increase light harvesting in a DSC seems to be the development of dyes that absorb with a high extinction coefficient across the targeted spectral range. In fact, recent record efficiencies stem from the development of organic porphyrin-based dyes with a donor- $\pi$-bridge-acceptor structure $[10,72]$. Yet, the absorption in high efficiency devices is not only determined by the intrinsic properties of the sensitiser but also influenced by the photonic design of the electrode structure. The photon path length $l$ can greatly exceed the film thickness through the incorporation of optical elements into the device structure. This is particularly important for the spectral regions with low extinction (typically in the red/infrared [5]) where the necessary film thickness would otherwise exceed rational device principles.

The incorporation of larger $\mathrm{TiO}_{2}$ particles to increase the photon path length by multiple scattering has been theoretically described as particularly beneficial with a wavelengthdependent optimum scatterer size of $d_{\text {iam }} \sim 0.68 \cdot \lambda[73,74]$. Nowadays, scattering particles of $\sim 400 \mathrm{~nm}$ diameter are a common element in record efficiency cells [9, 10, 13]. Various configurations have been developed, where the scattering particles are positioned either within or on top of the working electrode $[34,75]$. Recently, much progress has been made in the fabrication of mesoporous scattering particles of controlled size that can contribute to light absorption and electron generation due to their increased surface area $[76,77,78]$. While the implementation of scattering particles has proven highly beneficial for enhanced light absorption, the diffusive scattering over a broad spectral range has the detrimental effect of turning the cells opaque. This deprives the DSC of its variability in colour and thus, its potential for integrated architecture [29, 30].

An alternative concept that allows more detailed control over the optical action spectrum is the integration of photonic crystal (PC) elements into DSCs. These enable to enhance light harvesting in specific parts of the spectrum while preserving cell transparency in others $[36,79]$. 
Early experimental work on the integration of PC elements into the device architecture of DSCs was focussed on the use of 3D PCs in the form of $\mathrm{TiO}_{2}$ inverse opal structures $[80,81]$. These were deposited onto FTO substrates and subsequently covered with a mesoporous $\mathrm{TiO}_{2}$ layer of high surface area. PC fabrication limitations only allowed to deposit the mesoporous layer on top of the inverse opal structure, which required illumination from the counterelectrode to enable the penetration of incident light into the device structure. Nevertheless double layer devices showed a clear enhancement of the IPCE in the spectral vicinity of the photonic band gap. This was initially attributed to slow photon propagation $v_{\mathrm{g}}=\hbar \partial \omega / \partial k$ in the region of flat photon dispersion relation $E(k)[80,82]$. While this mechanism is in principle possible, it would only be effective for a narrow range of photon frequencies at the edge of the photonic band gap [83]. Further experimental investigations indicated that the absorption enhancement was mainly caused by incoherent scattering at disordered regions of the PC [81, 84]. Moreover, Bragg diffraction in the periodic lattice was identified as the third possible origin for the observed enhancement [85].

Mihi and Míguez later introduced a concept for the optimised coupling of a PC layer to a DSC [36], which is illustrated in Figure 3.5. According to their model, resonant cavities are formed between the PC top layer and the high refractive index TCE. The resonant modes are determined by the penetration depth of the incoming light into the PC and the thickness of the mesoporous film sandwiched between the PC and the substrate. These cavities give rise to the localisation of photons in the device, thus an enhanced absorption probability throughout the photonic band gap of the PC. The calculated optical response of the individual components with similar surface area is shown in Figure 3.5b for a sensitised 3D PC (black line) and mesoporous layer (dotted line). The expected absorption peak at the edge of the photonic band gap, caused by slow photon propagation in the 3D PC, can be clearly identified. However, the overall absorption of the 3D PC suffers from the impeded propagation of photons into the structure for frequencies within the photonic band gap. In contrast, a combined device architecture where a mesoporous layer is sandwiched between the TCE and the 3D PC is predicted to exhibit significant absorption enhancement in a wide spectral range, as shown in Figure 3.5c. Within the spectral region of the photonic band gap, the optical response oscillates between two situations, indicated as $\lambda_{1}$ and $\lambda_{2}$. Photons are either localised in the device due to the formation of a resonant cavity ( $\lambda_{1}$ : low reflection, high absorption, low group velocity) or simply reflected off the 3D PC ( $\lambda_{2}$ : high reflection, low absorption, high group velocity). In both cases, the result is an increased absorption in comparison to a reference DSC without an optical element due to the prolonged photon dwell time in the device.

While this device architecture is highly promising, the modelled effects strongly depend on the correct layer configuration and high structural order. Experimental realisation proved dif- 

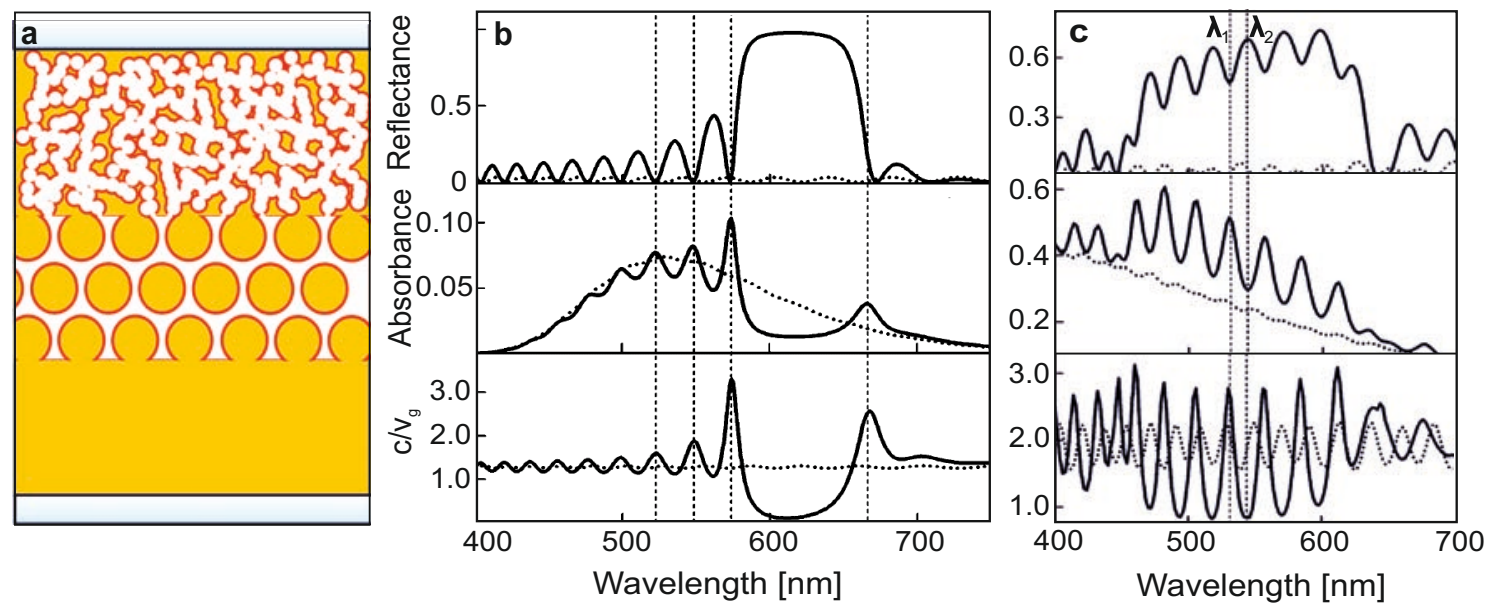

Figure 3.5: Integration of a 3D photonic crystal into the DSC device architecture. a) Schematic of a double layer photoanode, where a mesoporous $\mathrm{TiO}_{2}$ layer of high surface area is sandwiched between the FTO substrate and a highly ordered inverse opal layer, representing a 3D PC. b) Reflectance, absorptance and inverse group velocity (c/ $v_{\mathrm{g}}$ ) within the 3D PC (solid line) and in a conventional mesoporous film (dotted lines). c) Model calculation of the optical response of a double layer DSC electrode. Depending on the penetration depth into the 3D PC and the thickness of the mesoporous film, photons are either localised in the device due to the formation of a resonance cavity $\left(\lambda_{1}\right.$ : low reflection, high absorption, low group velocity) or simply reflected off the 3D PC ( $\lambda_{2}$ : high reflection, low absorption, high group velocity). Both cases lead to an absorption enhancement in comparison to a reference DSC without optical element, due to the prolonged photon dwell time in the device. b) - c) Adapted with permission from [36]. Copyright 2005 American Chemical Society.

ficult, mainly because of fabrication problems associated with the growth of a 3D inverse opal structure of high optical quality onto a previously deposited mesoporous $\mathrm{TiO}_{2}$. After evaporation induced self-assembly, the colloidal array had to be infiltrated with a high refractive index material, typically $\mathrm{TiO}_{2}$. This clogged the underlying mesoscopic pores and therefore inhibited sensitisation and electrolyte infiltration of the double layer device. The correct double layer configuration was finally experimentally achieved by protecting the mesoporous underlayer with a copolymer [86]. Yet, the lack of intimate physical contact between the two layers prevented the harvesting of PC-induced resonance effects.

An alternative to a 3D PC is the integration of a 1D PC into the device architecture, illustrated in Figure 3.6a. The 1D PC element is typically realised by a mesoporous Bragg reflector, which is fabricated via the cyclic deposition of nanoparticle-based $\mathrm{TiO}_{2}$ and $\mathrm{SiO}_{2}$ into a multilayer to achieve porosity and refractive index contrast in the stack [87]. The effect of the 1D PC on the light harvesting of the DSC has been experimentally and theoretically investigated $[88,79]$. The optical response of a double layer structure subject to illumination from the 
front side (white) or PC side (red) is shown in Figure 3.5e. Reference points are denoted at characteristic wavelengths $\lambda_{1}=775 \mathrm{~nm}$ (band-pass), $\lambda_{2}=575 \mathrm{~nm}$ (maximum reflectance) and $\lambda_{3}=512 \mathrm{~nm}$ (dip in reflection peak). The spatial distribution of the electric field $|E|^{2}$ under front side illumination, modelled by scalar wave approximation, is presented in Figure 3.5f. Similar to the case of a 3D PC, the concentration of the electric field throughout the photonic band gap of the 1D PC is evident. While $\lambda_{1}$ represents a reference region with almost no Bragg scattering by the PC top layer, $\lambda_{3}$ shows the effect of the formation of a resonance mode with an increase of $|E|_{\max }^{2}$ by a factor of $>2.5$. The overall benefit for the integration of a 1D PC in high performance DSCs was recently experimentally demonstrated [89, 90].
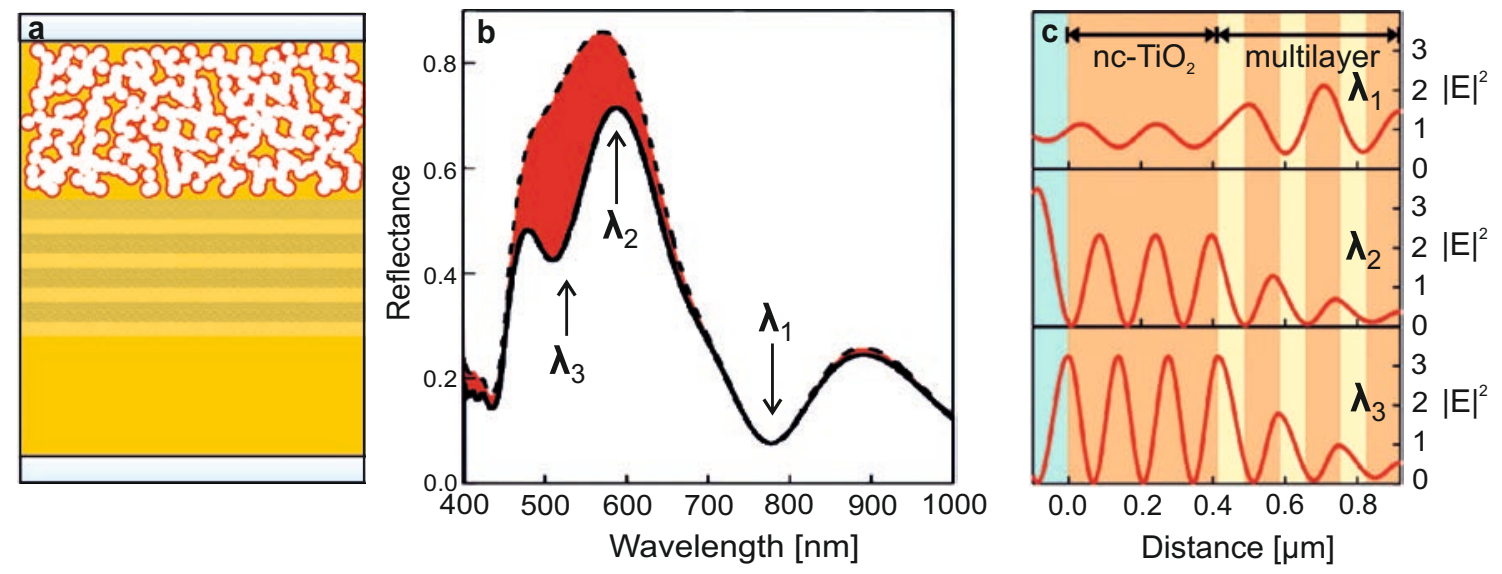

Figure 3.6: Integration of a 1D photonic crystal into the DSC device architecture. a) Schematic of a double layer device architecture with a mesoporous Bragg reflector acting as 1D PC. b) Comparison of optical response for a double layer DSC with illumination from the front side (white) and PC-side (red). c) Modelling of the electric field, based on scalar wave approximation, reveals the spatial distribution of $|E|^{2}$ in the double layer electrode depending on the wavelength of incoming photons, where $\lambda_{1}=775 \mathrm{~nm}$ (band-pass), $\lambda_{2}=575 \mathrm{~nm}$ (maximum reflectance) and $\lambda_{3}=512 \mathrm{~nm}$ (dip in reflection peak). b) - c) Adapted with permission from [88]. Copyright 2009 American Chemical Society.

\section{Bibliography}

[1] M. Grätzel, "Perspectives for dye-sensitized nanocrystalline solar cells," Progress In Photovoltaics, vol. 8, no. 1, pp. 171-185, 2000.

[2] J. Moser, "Notiz über die Verstärkung photoelektrischer Ströme durch optische Sensibilisierung," Monatshefte für Chemie, vol. 8, p. 373, 1887.

[3] B. O`Regan and M. Grätzel, "A low-cost, high-efficiency solar-cell based on dyesensitized colloidal $\mathrm{TiO}_{2}$ films," Nature, vol. 353, no. 6346, pp. 737-740, 1991.

[4] W. Shockley and H. Queisser, "Detailed balance limit of efficiency of p-n junction solar cells," Journal Of Applied Physics, vol. 32, no. 3, pp. 510-519, 1961. 
[5] M. Nazeeruddin, A. Kay, I. Rodicio, R. Humphry-Baker, E. Muller, P. Liska, N. Vlachopoulos, and M. Grätzel, "Conversion of light to electricity by cis-x2bis(2,2'bipyridyl-4,4'-dicarboxylate)ruthenium(ii) charge-transfer sensitizers (X = Cl-, Br-, I-, $\mathrm{Cn}-$, and Scn-) on nanocrystalline $\mathrm{TiO}_{2}$ electrodes," Journal Of The American Chemical Society, vol. 115, no. 14, pp. 6382-6390, 1993.

[6] M. Nazeeruddin, S. Zakeeruddin, R. Humphry-Baker, M. Jirousek, P. Liska, N. Vlachopoulos, V. Shklover, C. Fischer, and M. Grätzel, "Acid-base equilibria of (2,2 'bipyridyl-4,4 '-dicarboxylic acid)ruthenium(II) complexes and the effect of protonation on charge-transfer sensitization of nanocrystalline titania," Inorganic Chemistry, vol. 38, no. 26, pp. 6298-6305, 1999.

[7] M. Nazeeruddin, P. Pechy, T. Renouard, S. Zakeeruddin, R. Humphry-Baker, P. Comte, P. Liska, L. Cevey, E. Costa, V. Shklover, L. Spiccia, G. Deacon, C. Bignozzi, and M. Grätzel, "Engineering of efficient panchromatic sensitizers for nanocrystalline $\mathrm{TiO}_{2}-$ based solar cells," Journal Of The American Chemical Society, vol. 123, no. 8, pp. 1613$1624,2001$.

[8] M. Grätzel, "Dye-sensitized solar cells," Journal Of Photochemistry And Photobiology $C$, vol. 4, no. 2, pp. 145-153, 2003.

[9] Y. Chiba, A. Islam, Y. Watanabe, R. Komiya, N. Koide, and L. Han, "Dye-sensitized solar cells with conversion efficiency of 11.1\%," Japanese Journal Of Applied Physics Part 2-Letters $\mathcal{F}$ Express Letters, vol. 45, no. 24-28, pp. L638-L640, 2006.

[10] A. Yella, H.-W. Lee, H. Tsao, C. Yi, A. Chandiran, M. Nazeeruddin, E.-G. Diau, C.Y. Yeh, S. Zakeeruddin, and M. Grätzel, "Porphyrin-sensitized solar cells with cobalt (II/III)-based redox electrolyte exceed 12 percent efficiency," Science, vol. 334, no. 6056, pp. 629-634, 2011.

[11] J. Nelson, The physics of solar cells. Imperial College Press, 1st ed., 2003.

[12] "Terrestrial reference spectra for photovoltaic performance evaluation, g173-03," American Society for Testing and Materials (ASTM), http://rredc.nrel.gov/solar/spectra/am1.5, 1999.

[13] M. Nazeeruddin, T. Bessho, L. Cevey, S. Ito, C. Klein, F. De Angelis, S. Fantacci, P. Comte, P. Liska, H. Imai, and M. Grätzel, "A high molar extinction coefficient charge transfer sensitizer and its application in dye-sensitized solar cell," Journal Of Photochemistry And Photobiology A-Chemistry, vol. 185, no. 2-3, pp. 331-337, 2007.

[14] A. Hagfeldt and M. Grätzel, "Molecular photovoltaics," Accounts Of Chemical Research, vol. 33, no. 5, pp. 269-277, 2000.

[15] G. Benko, J. Kallioinen, J. Korppi-Tommola, A. Yartsev, and V. Sundstrom, "Photoinduced ultrafast dye-to-semiconductor electron injection from nonthermalized and thermalized donor states," Journal Of The American Chemical Society, vol. 124, no. 3, pp. 489-493, 2002.

[16] P. Barnes, L. Liu, X. Li, A. Y. Anderson, H. Kisserwan, T. Ghaddar, J. Durrant, and B. O'Regan, "Re-evaluation of recombination losses in dye-sensitized cells: the failure of dynamic relaxation methods to correctly predict diffusion length in nanoporous photoelectrodes," Nano Letters, vol. 9, no. 10, pp. 3532-3538, 2009. 
[17] Q. Wang, S. Ito, M. Grätzel, F. Fabregat-Santiago, I. Mora-Sero, J. Bisquert, T. Bessho, and H. Imai, "Characteristics of high efficiency dye-sensitized solar cells," Journal Of Physical Chemistry B, vol. 110, no. 50, pp. 25210-25221, 2006.

[18] S. Haque, Y. Tachibana, D. Klug, and J. Durrant, "Charge recombination kinetics in dyesensitized nanocrystalline titanium dioxide films under externally applied bias," Journal Of Physical Chemistry B, vol. 102, no. 10, pp. 1745-1749, 1998.

[19] A. Hagfeldt, G. Boschloo, L. Sun, L. Kloo, and H. Pettersson, "Dye-Sensitized Solar Cells," Chemical Reviews, vol. 110, no. 11, pp. 6595-6663, 2010.

[20] A. Hauch and A. Georg, "Diffusion in the electrolyte and charge-transfer reaction at the platinum electrode in dye-sensitized solar cells," Electrochimica Acta, vol. 46, no. 22, pp. 3457-3466, 2001.

[21] N. Papageorgiou, W. Maier, and M. Grätzel, "An iodine/triiodide reduction electrocatalyst for aqueous and organic media," Journal Of The Electrochemical Society, vol. 144, no. 3, pp. 876-884, 1997.

[22] S. Haque, Y. Tachibana, R. Willis, J. Moser, M. Grätzel, D. Klug, and J. Durrant, "Parameters influencing charge recombination kinetics in dye-sensitized nanocrystalline titanium dioxide films," Journal Of Physical Chemistry B, vol. 104, no. 3, pp. 538-547, 2000 .

[23] J. van de Lagemaat, N. Park, and A. Frank, "Influence of electrical potential distribution, charge transport, and recombination on the photopotential and photocurrent conversion efficiency of dye-sensitized nanocrystalline $\mathrm{TiO}_{2}$ solar cells: a study by electrical impedance and optical modulation techniques," Journal Of Physical Chemistry B, vol. 104, no. 9, pp. 2044-2052, 2000.

[24] A. Zaban, M. Greenshtein, and J. Bisquert, "Determination of the electron lifetime in nanocrystalline dye solar cells by open-circuit voltage decay measurements," ChemPhysChem, vol. 4, no. 8, pp. 859-864, 2003.

[25] H. Snaith and L. Schmidt-Mende, "Advances in liquid-electrolyte and solid-state dyesensitized solar cells," Advanced Materials, vol. 19, no. 20, pp. 3187-3200, 2007.

[26] M. Grätzel, "Photoelectrochemical cells," Nature, vol. 414, no. 6861, pp. 338-344, 2001.

[27] M. Grätzel, "Recent Advances in Sensitized Mesoscopic Solar Cells," Accounts Of Chemical Research, vol. 42, no. 11, pp. 1788-1798, 2009.

[28] J. Kroon, N. Bakker, H. Smit, P. Liska, K. Thampi, P. Wang, S. Zakeeruddin, M. Grätzel, A. Hinsch, S. Hore, U. Würfel, R. Sastrawan, J. Durrant, E. Palomares, H. Pettersson, T. Gruszecki, J. Walter, K. Skupien, and G. Tulloch, "Nanocrystalline dye-sensitized solar cells having maximum performance," Progress In Photovoltaics, vol. 15, no. 1, pp. 1-18, 2007.

[29] M. Grätzel, "The advent of mesoscopic injection solar cells," Progress In Photovoltaics, vol. 14, no. 5, pp. 429-442, 2006.

[30] S. Yoon, S. Tak, J. Kim, Y. Jun, K. Kang, and J. Park, "Application of transparent dyesensitized solar cells to building integrated photovoltaic systems," Building And Environment, vol. 46, no. 10, pp. 1899-1904, 2011. 
[31] H. Snaith, "Estimating the maximum attainable efficiency in dye-sensitized solar cells," Advanced Functional Materials, vol. 20, no. 1, pp. 13-19, 2010.

[32] L. Peter, "Dye-sensitized nanocrystalline solar cells," Physical Chemistry Chemical Physics, vol. 9, no. 21, pp. 2630-2642, 2007.

[33] G. Boschloo and A. Hagfeldt, "Characteristics of the Iodide/Triiodide Redox Mediator in Dye-Sensitized Solar Cells," Accounts Of Chemical Research, vol. 42, no. 11, pp. 18191826, 2009.

[34] Z. Wang, H. Kawauchi, T. Kashima, and H. Arakawa, "Significant influence of $\mathrm{TiO}_{2}$ photoelectrode morphology on the energy conversion efficiency of N719 dye-sensitized solar cell," Coordination Chemistry Reviews, vol. 248, no. 13-14, pp. 1381-1389, 2004.

[35] J.-H. Yum, E. Baranoff, S. Wenger, M. Nazeeruddin, and M. Grätzel, "Panchromatic engineering for dye-sensitized solar cells," Energy $\mathcal{E}$ Environmental Science, vol. 4, no. 3, pp. 842-857, 2011.

[36] A. Mihi and H. Míguez, "Origin of light-harvesting enhancement in colloidal-photoniccrystal-based dye-sensitized solar cells," Journal Of Physical Chemistry B, vol. 109, no. 33, pp. 15968-15976, 2005.

[37] M. Calvo, S. Colodrero, N. Hidalgo, G. Lozano, C. Lopez-Lopez, O. Sanchez-Sobrado, and H. Míguez, "Porous one dimensional photonic crystals: novel multifunctional materials for environmental and energy applications," Energy $\mathcal{E}$ Environmental Science, vol. 4, pp. 4800-4812, 2011.

[38] P. Cameron, L. Peter, and S. Hore, "How important is the back reaction of electrons via the substrate in dye-sensitized nanocrystalline solar cells?" Journal Of Physical Chemistry B, vol. 109, no. 2, pp. 930-936, 2005.

[39] B. O'Regan and J. Durrant, "Kinetic and energetic paradigms for dye-sensitized solar cells: moving from the ideal to the real.," Accounts of chemical research, vol. 42, no. 11, pp. 1799-808, 2009.

[40] T. Dittrich, E. Lebedev, and J. Weidmann, "Electron drift mobility in porous $\mathrm{TiO}_{2}$ (anatase)," Physica Status Solidi A, vol. 1998, no. 165, pp. R5-R6, 1998.

[41] S. Burnside, V. Shklover, C. Barbé, P. Comte, F. Arendse, K. Brooks, and M. Grätzel, "Self-organization of $\mathrm{TiO}_{2}$ nanoparticles in thin films," Chemistry Of Materials, vol. 10, no. 9, pp. 2419-2425, 1998.

[42] H. Tang, K. Prasad, R. Sanjines, P. Schmid, and F. Levy, "Electrical and optical properties of $\mathrm{TiO}_{2}$ anatase thin-films," Journal Of Applied Physics, vol. 75, no. 4, pp. 2042-2047, 1994.

[43] P. Tiwana, P. Parkinson, M. Johnston, H. Snaith, and L. Herz, "Ultrafast terahertz conductivity dynamics in mesoporous $\mathrm{TiO}_{2}$ : influence of dye sensitization and surface treatment in solid-state dye-sensitized solar cells," Journal Of Physical Chemistry C, vol. 114, no. 2 , pp. 1365-1371, 2010.

[44] P. Tiwana, P. Docampo, M. Johnston, H. Snaith, and L. Herz, "Electron mobility and injection dynamics in mesoporous $\mathrm{ZnO}, \mathrm{SnO}_{2}$, and $\mathrm{TiO}_{2}$ films used in dye-sensitized solar cells," ACS Nano, vol. 5, no. 6, pp. 5158-5166, 2011. 
[45] L. Forro, O. Chauvet, D. Emin, L. Zuppiroli, H. Berger, and F. Levy, "High-mobility n-type charge-carriers in large single-crystals of anatase $\left(\mathrm{TiO}_{2}\right)$," Journal Of Applied Physics, vol. 75, no. 1, pp. 633-635, 1994.

[46] A. Frank, N. Kopidakis, and J. van de Lagemaat, "Electrons in nanostructured $\mathrm{TiO}_{2}$ solar cells: transport, recombination and photovoltaic properties," Coordination Chemistry Reviews, vol. 248, no. 13-14, pp. 1165-1179, 2004.

[47] J. Bisquert, "Chemical diffusion coefficient of electrons in nanostructured semiconductor electrodes and dye-sensitized solar cells," Journal Of Physical Chemistry B, vol. 108, no. 7, pp. 2323-2332, 2004.

[48] J. van de Lagemaat, K. Zhu, K. Benkstein, and A. Frank, "Temporal evolution of the electron diffusion coefficient in electrolyte-filled mesoporous nanocrystalline $\mathrm{TiO}_{2}$ films," Inorganica Chimica Acta, vol. 361, no. 3, pp. 620-626, 2008.

[49] L. Dloczik, O. Ileperuma, I. Lauermann, L. Peter, E. Ponomarev, G. Redmond, N. Shaw, and I. Uhlendorf, "Dynamic response of dye-sensitized nanocrystalline solar cells: characterization by intensity-modulated photocurrent spectroscopy," Journal Of Physical Chemistry B, vol. 101, no. 49, pp. 10281-10289, 1997.

[50] N. Kopidakis, N. Neale, K. Zhu, J. van de Lagemaat, and A. Frank, "Spatial location of transport-limiting traps in $\mathrm{TiO}_{2}$ nanoparticle films in dye-sensitized solar cells," Applied Physics Letters, vol. 87, no. 20, 2005.

[51] K. Zhu, N. Kopidakis, N. Neale, J. van de Lagemaat, and A. Frank, "Influence of surface area on charge transport and recombination in dye-sensitized $\mathrm{TiO}_{2}$ solar cells," Journal Of Physical Chemistry B, vol. 110, no. 50, pp. 25174-25180, 2006.

[52] M. Adachi, Y. Murata, J. Takao, J. Jiu, M. Sakamoto, and F. Wang, "Highly efficient dyesensitized solar cells with a titania thin-film electrode composed of a network structure of single-crystal-like $\mathrm{TiO}_{2}$ nanowires made by the "oriented attachment" mechanism," Journal Of The American Chemical Society, vol. 126, no. 45, pp. 14943-14949, 2004.

[53] K. Benkstein, N. Kopidakis, J. van de Lagemaat, and A. Frank, "Influence of the percolation network geometry on electron transport in dye-sensitized titanium dioxide solar cells," Journal Of Physical Chemistry B, vol. 107, no. 31, pp. 7759-7767, 2003.

[54] U. Bach, D. Lupo, P. Comte, J. E. Moser, F. Weissörtel, J. Salbeck, H. Spreitzer, and M. Grätzel, "Solid-state dye-sensitized mesoporous $\mathrm{TiO}_{2}$ solar cells with high photonto-electron conversion efficiencies," Nature, vol. 395, pp. 583-585, 1998.

[55] J. Burschka, A. Dualeh, F. Kessler, E. Baranoff, N.-L. Cevey-Ha, C. Yi, M. Nazeeruddin, and M. Grätzel, "Tris(2-(1H-pyrazol-1-yl)pyridine)cobalt(III) as p-type dopant for organic semiconductors and its application in highly efficient solid-state dye-sensitized solar cells," Journal Of The American Chemical Society, vol. 133, no. 45, pp. 1804218045, 2011.

[56] N. Tetreault and M. Grätzel, "Novel nanostructures for next generation dye-sensitized solar cells," Energy E Environmental Science, DOI: 10.1039/C2EE03242B, 2012.

[57] L. Schmidt-Mende and M. Grätzel, " $\mathrm{TiO}_{2}$ pore-filling and its effect on the efficiency of solid-state dye-sensitized solar cells," Thin Solid Films, vol. 500, no. 1-2, pp. 296-301, 2006. 
[58] J. Melas-Kyriazi, I.-K. Ding, A. Marchioro, A. Punzi, B. Hardin, G. Burkhard, N. Tetreault, M. Grätzel, J.-E. Moser, and M. McGehee, "The effect of hole transport material pore filling on photovoltaic performance in solid-state dye-sensitized solar cells," Advanced Energy Materials, vol. 1, no. 3, pp. 407-414, 2011.

[59] N. Tetreault, E. Horvath, T. Moehl, J. Brillet, R. Smajda, S. Bungener, N. Cai, P. Wang, S. Zakeeruddin, L. Forro, A. Magrez, and M. Grätzel, "High-efficiency solid-state dyesensitized solar cells: fast charge extraction through self-assembled 3D fibrous network of crystalline $\mathrm{TiO}_{2}$ nanowires," ACS Nano, vol. 4, no. 12, pp. 7644-7650, 2010.

[60] X. Feng, K. Shankar, O. Varghese, M. Paulose, T. Latempa, and C. Grimes, "Vertically aligned single crystal $\mathrm{TiO}_{2}$ nanowire arrays grown directly on transparent conducting oxide coated glass: synthesis details and applications," Nano Letters, vol. 8, no. 11, pp. 3781-3786, 2008.

[61] B. Liu and E. Aydil, "Growth of oriented single-crystalline rutile $\mathrm{TiO}_{2}$ nanorods on transparent conducting substrates for dye-sensitized solar cells," Journal Of The American Chemical Society, vol. 131, no. 11, pp. 3985-3990, 2009.

[62] O. Varghese, M. Paulose, and C. Grimes, "Long vertically aligned titania nanotubes on transparent conducting oxide for highly efficient solar cells," Nature Nanotechnology, vol. 4, no. 9, pp. 592-597, 2009.

[63] M. Law, L. Greene, J. Johnson, R. Saykally, and P. Yang, "Nanowire dye-sensitized solar cells," Nature Materials, vol. 4, no. 6, pp. 455-459, 2005.

[64] R. Penn and J. Banfield, "Morphology development and crystal growth in nanocrystalline aggregates under hydrothermal conditions: Insights from titania," Geochimica Et Cosmochimica Acta, vol. 63, no. 10, pp. 1549-1557, 1999.

[65] F. Sauvage, F. Di Fonzo, A. Bassi, C. Casari, V. Russo, G. Divitini, C. Ducati, C. Bottani, P. Comte, and M. Grätzel, "Hierarchical $\mathrm{TiO}_{2}$ photoanode for dye-sensitized solar cells," Nano Letters, vol. 10, no. 7, pp. 2562-2567, 2010.

[66] I. Cho, Z. Chen, A. Forman, D. Kim, P. Rao, T. Jaramillo, and X. Zheng, "Branched $\mathrm{TiO}_{2}$ nanorods for photoelectrochemical hydrogen production," Nano Letters, vol. 11, no. 11, pp. 4978-4984, 2011.

[67] E. Enache-Pommer, B. Liu, and E. Aydil, "Electron transport and recombination in dyesensitized solar cells made from single-crystal rutile $\mathrm{TiO}_{2}$ nanowires," Physical Chemistry Chemical Physics, vol. 11, no. 42, pp. 9648-9652, 2009.

[68] K. Zhu, N. Neale, A. Miedaner, and A. Frank, "Enhanced charge-collection efficiencies and light scattering in dye-sensitized solar cells using oriented $\mathrm{TiO}_{2}$ nanotubes arrays," Nano Letters, vol. 7, no. 1, pp. 69-74, 2007.

[69] K. Zhu, N. Neale, A. Halverson, J. Kim, and A. Frank, "Effects of annealing temperature on the charge-collection and light-harvesting properties of $\mathrm{TiO}_{2}$ nanotube-based dyesensitized solar cells," Journal Of Physical Chemistry C, vol. 114, no. 32, pp. 1343313441, 2010.

[70] E. Crossland, M. Kamperman, M. Nedelcu, C. Ducati, U. Wiesner, D.-M. Smilgies, G. Toombes, M. Hillmyer, S. Ludwigs, U. Steiner, and H. Snaith, "A bicontinuous double gyroid hybrid solar cell," Nano Letters, vol. 9, no. 8, pp. 2807-2812, 2009. 
[71] E. Crossland, M. Nedelcu, C. Ducati, S. Ludwigs, M. Hillmyer, U. Steiner, and H. Snaith, "Block copolymer morphologies in dye-sensitized solar cells: probing the photovoltaic structure-function relation," Nano Letters, vol. 9, no. 8, pp. 2813-2819, 2009.

[72] N. Cai, S.-J. Moon, L. Cevey-Ha, T. Moehl, R. Humphry-Baker, P. Wang, S. Zakeeruddin, and M. Grätzel, "An organic D-pi-A dye for record efficiency solid-state sensitized heterojunction solar cells," Nano Letters, vol. 11, no. 4, pp. 1452-1456, 2011.

[73] A. Usami, "Theoretical study of application of multiple scattering of light to a dye-sensitized nanocrystalline photoelectrochemical cell," Chemical Physics Letters, vol. 277, no. 1-3, pp. 105-108, 1997.

[74] J. Ferber and J. Luther, "Computer simulations of light scattering and absorption in dyesensitized solar cells," Solar Energy Materials And Solar Cells, vol. 54, no. 1-4, pp. 265$275,1998$.

[75] S. Hore, C. Vetter, R. Kern, H. Smit, and A. Hinsch, "Influence of scattering layers on efficiency of dye-sensitized solar cells," Solar Energy Materials and Solar Cells, vol. 90, no. 9, pp. 1176-1188, 2006.

[76] F. Sauvage, D. Chen, P. Comte, F. Huang, L.-P. Heiniger, Y.-B. Cheng, R. A. Caruso, and M. Grätzel, "Dye-sensitized solar cells employing a single film of mesoporous $\mathrm{TiO}_{2}$ beads achieve power conversion efficiencies over 10\%.", ACS Nano, vol. 4, no. 8, pp. 4420-5, 2010.

[77] B. Chen, F. Huang, Y.-B. Cheng, and R. Caruso, "Mesoporous anatase $\mathrm{TiO}_{2}$ beads with high surface areas and controllable pore sizes : a superior candidate for high-performance dye-sensitized solar cells," Advanced Materials, vol. 21, pp. 2206-2210, 2009.

[78] I. Yu, Y. Kim, H. Kim, and W. Lee, C.And Lee, "Size-dependent light-scattering effects of nanoporous $\mathrm{TiO}_{2}$ spheres in dye-sensitized solar cells," Journal Of Materials Chemistry, vol. 21, no. 2, pp. 532-538, 2011.

[79] D. Colonna, S. Colodrero, H. Lindstrom, A. Di Carlo, and H. Míguez, "Introducing structural colour in dscs by using photonic crystals: interplay between conversion efficiency and optical properties," Energy $\mathcal{F}$ Environmental Science, DOI: 10.1039/C2EE02658A, 2012.

[80] S. Nishimura, N. Abrams, B. Lewis, L. Halaoui, T. Mallouk, K. Benkstein, J. van de Lagemaat, and A. Frank, "Standing wave enhancement of red absorbance and photocurrent in dye-sensitized titanium dioxide photoelectrodes coupled to photonic crystals," Journal of the American Chemical Society, vol. 125, no. 3, pp. 6306-6310, 2003.

[81] L. Halaoui, N. Abrams, and T. Mallouk, "Increasing the conversion efficiency of dyesensitized $\mathrm{TiO}_{2}$ photoelectrochemical cells by coupling to photonic crystals," Journal of Physical Chemistry B, vol. 109, no. 13, pp. 6334-6342, 2005.

[82] K. Sakoda, "Enhanced light amplification due to group-velocity anomaly peculiar to twoand three-dimensional photonic crystals," Optics Express, vol. 4, no. 5, pp. 167-176, 1999.

[83] G. von Freymann, S. John, S. Wong, V. Kitaev, and G. Ozin, "Measurement of group velocity dispersion for finite size three-dimensional photonic crystals in the near-infrared spectral region," Applied Physics Letters, vol. 86, no. 5, 2005. 
[84] R. Rengarajan, D. Mittleman, C. Rich, and V. Colvin, "Effect of disorder on the optical properties of colloidal crystals," Physical Review E, vol. 71, no. 1, Part 2, pp. 1596815976, 2005.

[85] D. Mittleman, J. Bertone, P. Jiang, K. Hwang, and V. Colvin, "Optical properties of planar colloidal crystals: dynamical diffraction and the scalar wave approximation," Journal of Chemical Physics, vol. 111, no. 1, pp. 345-354, 1999.

[86] S.-H. A. Lee, N. Abrams, P. Hoertz, G. Barber, L. Halaoui, and T. Mallouk, "Coupling of titania inverse opals to nanocrystalline titania layers in dye-sensitized solar cells," Journal Of Physical Chemistry B, vol. 112, no. 46, pp. 14415-14421, 2008.

[87] S. Colodrero, M. Ocaña, A. Gonzalez-Elipe, and H. Míguez, "Response of nanoparticlebased one-dimensional photonic crystals to ambient vapor pressure," Langmuir, vol. 24, no. 21, pp. 9135-9139, 2008.

[88] S. Colodrero, A. Mihi, J. Anta, M. Ocana, and H. Míguez, "Experimental demonstration of the mechanism of light harvesting enhancement in photonic-crystal-based dyesensitized solar cells," Journal Of Physical Chemistry C, vol. 113, no. 4, pp. 1150-1154, 2009.

[89] S. Colodrero, A. Mihi, L. Haggman, M. Ocaña, G. Boschloo, A. Hagfeldt, and H. Míguez, "Porous one-dimensional photonic crystals improve the power-conversion efficiency of dye-sensitized solar cells," Advanced Materials, vol. 21, no. 7, p. 764770, 2009.

[90] S. Colodrero, A. Forneli, C. Lopez-Lopez, L. Pelleja, H. Míguez, and E. Palomares, "Efficient Transparent Thin Dye Solar Cells Based on Highly Porous 1D Photonic Crystals," Advanced Functional Materials, vol. 22, no. 6, pp. 1303-1310, 2012. 


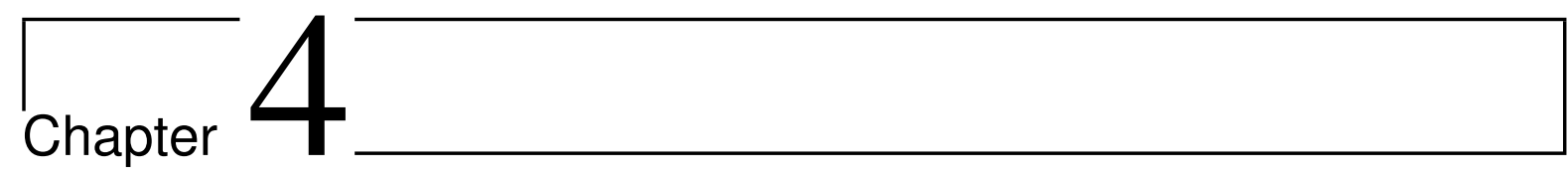

\section{Experimental and analytical techniques}

\subsection{Materials fabrication}

\subsubsection{Poly(isoprene-block-ethylene oxide) copolymers}

Poly(isoprene-block-ethylene oxide) (PI- $b$-PO) exhibits several intrinsic characteristics which make the block copolymer (BCP) system excellently suited as a structure-directing agent for the co-assembly of inorganic nanostructures. Polar hydrolysis products of titanium or siliconbased precursors preferentially reside in the hydrophilic PEO block due to the formation of hydrogen bonds with the ethylene oxide [1]. The Flory-Huggins interaction parameter of the PI-PEO couple is particularly high compared to other systems [2]. The low glass transition temperature of $213 \mathrm{~K}$ for both blocks allows high chain mobilities at ambient temperatures $[3,4]$. Anionic polymerisation enables the synthesis of molecules with a high degree of polymerisation and low polydispersities [5]. The self-assembly behaviour of PI- $b$-PEO has been widely studied intensively and its phase diagram is now well understood, covering the full range of PI/PEO volume ratios [6]. Besides several PI- $b$-PEO diblock copolymers, a triblock terpolymer PI- $b$-PS- $b$-PEO with an intermediate PS (polystyrene) block was also used in the course of this thesis. See Table 4.1 for an overview of the employed BCPs.

All BCPs employed in this study were synthesised and characterised by Morgan Stefik and Juho Song in the group of Professor Ulrich Wiesner at Cornell University, USA. See reference [5] for experimental details. The polymers were characterised by ${ }^{1} \mathrm{H}$ NMR spectroscopy and their polydispersity was determined using gel permeation chromatography (GPC). The characteristic properties of polymers used in this study are shown in Table 4.1. 
Table 4.1: Characteristic properties of employed BCPs.

\begin{tabular}{cccccc}
\hline $\begin{array}{c}M_{\mathrm{n}} \text { PI- } b-\mathrm{PEO} \\
{[\mathrm{kg} / \mathrm{mol}]}\end{array}$ & $N_{\text {TOT }}$ & $\begin{array}{c}M_{\mathrm{n}} \text { PEO } \\
{[\mathrm{kg} / \mathrm{mol}]}\end{array}$ & $N_{\mathrm{PEO}}$ & $\begin{array}{c}f_{\mathrm{PEO}} \\
{[\%]}\end{array}$ & PDI $\left(=\frac{M_{\mathrm{w}}}{M_{\mathrm{n}}}\right)$ \\
\hline 12.5 & 204 & 2.6 & 58 & 20.4 & 1.10 \\
34.4 & 606 & 9.8 & 245 & 28.4 & 1.05 \\
35.7 & 609 & 8.2 & 205 & 23.0 & 1.03 \\
91.6 & 1579 & 28.6 & 651 & 31.2 & 1.09 \\
\hline \multicolumn{7}{c}{} & & & & \\
\hline$M_{\mathrm{n}}$ PI- $b$-PS- $b-\mathrm{PEO}$ & $M_{\mathrm{n}}$ PI & $M_{\mathrm{n}}$ PS & $M_{\mathrm{n}}$ PEO & $f_{\mathrm{PEO}}$ & PDI \\
{$[\mathrm{kg} / \mathrm{mol}]$} & {$[\mathrm{kg} / \mathrm{mol}]$} & {$[\mathrm{kg} / \mathrm{mol}]$} & {$[\mathrm{kg} / \mathrm{mol}]$} & {$[\%]$} & \\
\hline 53.4 & 14.6 & 29.0 & 9.8 & 19.4 & 1.05 \\
\hline \multicolumn{7}{c}{} \\
\hline
\end{tabular}

\subsubsection{Sol-gel chemistry}

Hydrolytic sol-gel chemistry is a solution-based technique that leads to the formation of an oxide, like $\mathrm{SiO}_{2}, \mathrm{TiO}_{2}$ or $\mathrm{Nb}_{2} \mathrm{O}_{5}$, via a two-step chemical reaction. This involves the hydrolysis of the precursor material (typically a metal alkoxide or metal salt):

$$
\mathrm{X}-(\mathrm{OR})_{v}+\mathrm{H}_{2} \mathrm{O} \rightarrow \mathrm{OH}-\mathrm{X}-\mathrm{OR}_{v-1}+\mathrm{ROH}
$$

where $\mathrm{X}$ represents the core chemical element of the precursor material, $v$ its valency and $\mathrm{R}$ is the organic substituent. The fully hydrolysed precursor $\mathrm{X}-(\mathrm{OH})_{v}$ in a colloidal suspension (sol) then undergoes condensation reactions to form an oxide network (gel) via one of the two chemical pathways:

$$
\begin{aligned}
\mathrm{X}-\mathrm{OH}_{v}+\mathrm{X}-\mathrm{OH}_{v} \rightarrow & \mathrm{OH}_{v-1}-\mathrm{XOX}-\mathrm{OH}_{v-1}+\mathrm{H}_{2} \mathrm{O} \\
\mathrm{X}-\mathrm{OR}_{v}+\mathrm{X}-\mathrm{OH}_{v} \rightarrow \mathrm{OH}_{v-1} \mathrm{XOX}-\mathrm{OR}_{v-1}+\mathrm{ROH} & \text { et sequens }
\end{aligned}
$$

Depending on the reaction kinetics, condensation may also take place between partially hydrolysed species, resulting in a multitude of different reaction sequences [7]. Sol-gel chemistry is an effective chemical route for the BCP-directed assembly of inorganic material if the reaction kinetics can be sufficiently controlled. This includes limitations in spatial dimensions of the guest material to allow incorporation into one of the BCP phases [8]. Furthermore, the gelation process needs to be slow in comparison with the time scale for microphase separation to prevent quenching of the $\mathrm{BCP}$-driven structure formation process before reaching thermodynamic equilibrium [9]. Another challenge is the sol-gel accompanied release of wa- 
ter and alcohol, as this may impede the symmetric evaporation of solvents from hydrophilic and hydrophobic components. A major advantage of the sol-gel route is the possibility to fully condense the inorganic material into a homogeneous and continuous network, e.g. through temperature annealing after microphase separation, which distinguishes the technique from co-assembly of chemically inert nanoparticles.

\section{Hydrolytic sol-gel chemistry of $\mathrm{TiO}_{2}$}

In contrast to silica sol-gel chemistry, where the hydrolysis of a silicon alkoxide typically needs the catalysis by an electrophile, the hydrolysis and subsequent condensation of metal alkoxides occurs spontaneously and is difficult to control [7]. Several strategies have been introduced to overcome the problem of uncontrolled condensation and macroscopic phase separation, including the utilisation of $\mathrm{pH}$ variation, stabilising ligands, nonaqueous media and preformed nanoclusters [10]. Yet, the kinetic control is still restricted, preventing a comparable structure control and polymorphism as reported in neat $\mathrm{BCP}$ or $\mathrm{BCP} /$ silica systems $[10,11]$. In this work the titanium alkoxides titanium isopropoxide $\left(\mathrm{OR} \hat{=} \mathrm{OCH}\left(\mathrm{CH}_{3}\right)_{2}\right)$ and titanium ethoxide $\left(\mathrm{OR} \hat{=} \mathrm{OCH}_{2} \mathrm{CH}_{3}\right)$ were used as titanium source. Control of reaction kinetics was attempted by manipulation of the $\mathrm{pH}$, ratio of water molecules and detachment of hydrolysis and phase separation by the introduction of a solvent exchange.

\section{Hydrolytic sol-gel chemistry of aluminosilicates}

The co-assembly of organically modified aluminosilicates has been of interest in this work due to its abrasion resistance and low refractive index [12]. The sol-gel chemistry of aluminosilica involves the hydrolysis and condensation of a mixture of silicon and aluminium precursors, typically in a molar ratio of 9:1 ( $\mathrm{Si}: \mathrm{Al})$. In the course of this study a sol-gel route was employed, where aluminum sec-butoxide serves as the aluminum source and 3glycidyloxypropyltrimethoxysilane as the silicon source. The organic modification 3-glycidyloxypropyl plays an important role in the solubility and compatibilisation of the inorganic material during the subsequent co-assembly process [13]. Due to the reactivity of the aluminum sec-butoxide, the mixture is typically hydrolysed in a two-step process, where the first $15 \%$ of the final amount of acidic water is added at $0{ }^{\circ} \mathrm{C}$. The mixture of aluminium and silicon in the aluminosilica material then occurs via the hydrolysed precursors. According to ${ }^{27} \mathrm{Al}$ solid state nuclear magnetic resonance spectroscopy, the aluminium is typically found in tetrahedral and octahedral coordination, i.e. evenly distributed in the silicon-framework and in aluminium microdomains, respectively [14]. 


\subsubsection{Thin film deposition}

The fabrication of BCP-derived material architectures in thin films is inherently linked to solution-based processing. Polymers and inorganic material are typically mixed in a solvent system which is compatible with all components. Self-organisation into a phase separated film then occurs during the evaporation of the solvent. The evaporation rate of the solvent system therefore defines the available time-scale for structure formation [15].

\section{Spin coating}

Spin coating deposits a solute material onto a substrate by angular rotation. Typically an excess volume of solution is placed onto a substrate, which is held on a vacuum stage. The stage is then accelerated up to a rotational speed between 500 and 10,000 revolutions per minute. During the spinning process the droplet develops in a three step process. In a first phase most solution is centrifuged off the substrate to form a homogeneous film of thickness $h_{0}$ and a concentration $c_{0}$. Outflow of solution dominates the mass loss of the sample in the second phase until a critical film thickness $h_{c}$ is reached. In the final phase the evaporation of solvents becomes the dominant mechanism of mass loss, leading to a dry film of defined thickness, typically in the range of $10 \mathrm{~nm}$ to several $\mu \mathrm{m}[16,17]$. A schematic of the spin coating procedure is shown in Figure 4.1a. The resulting final film thickness $h_{\infty}$ can be calculated as a function of rotational speed $\omega$, viscosity $\eta$ and initial concentration $c_{0}$, which has been verified experimentally for model systems [17, 18].

$$
h_{\infty} \sim \omega^{-1 / 2} \eta^{1 / 3} c_{0} .
$$
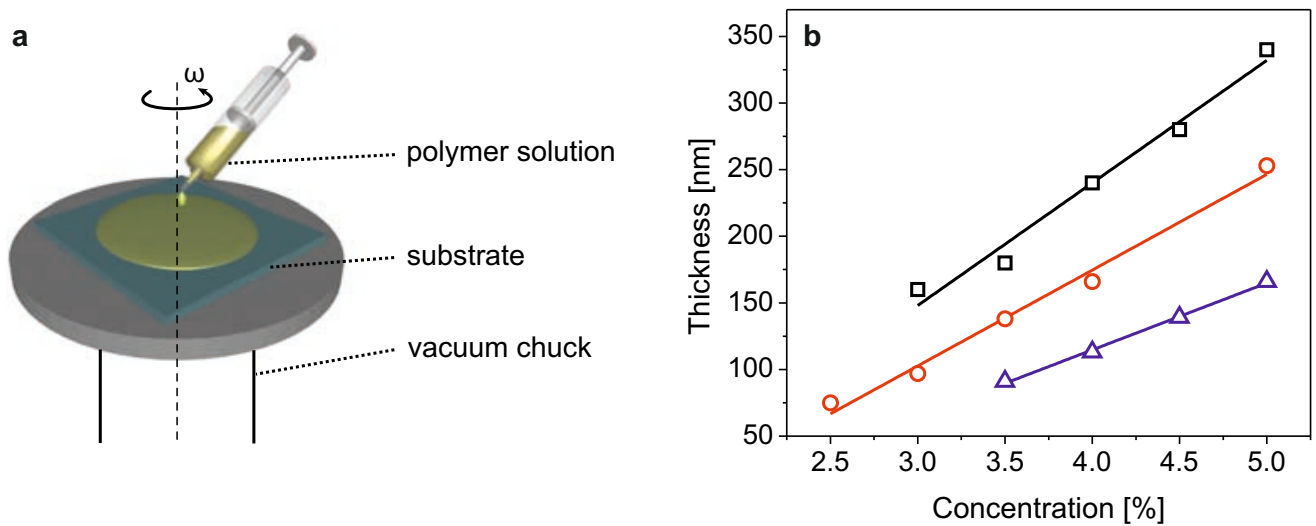

Figure 4.1: Spin coating and film thickness calibration. a) Schematic of the spin coating process, where a substrate is held on a vacuum stage and covered by excess volume of solution before the stage is accelerated to a rotational speed between 500 and 10,000 revolutions per minute for film formation. b) Exemplary relationship between final film thickness and solute concentration for different solvent systems. a): Adapted with permission from [19]. Copyright Mathias Kolle. 
The linear dependence of the final film thickness on the initial concentration of the solution is in good agreement with results in this work, presented in Figure 4.1b. The adjustment of concentration was therefore used as the main parameter for film thickness control. Spin coating was carried out on a WS-400B-6NPP-Lite processor (Laurell Technologies).

\section{Blade coating}

Blade coating (or doctor blading) is an alternative technique for thin film deposition on the lab-scale with accessible film thicknesses ranging from several hundred $\mathrm{nm}$ to $\mu \mathrm{m}$. Here, a drop of solution is placed in front of a sharp blade that is then drawn linearly across the substrate to form a thin wet layer, which subsequently dries into a solid film. The thickness of the film can be controlled by the vertical distance between the substrate and the blade, the concentration of the solution and the drying kinetics. Another important parameter is the shape of the evolving meniscus, which depends on the surface energy of the substrate, surface tension of the solution, its viscosity and the shear field that is a function of the drawing speed [20]. The employed set-up, used in the course of this work, is shown in Figure 4.2. A commercially available instrument (RK Print Coat Instruments, K Paint Applicator) was extended by a solvent-fed chamber and a programmable heating element (Omron E5CS). The enclosed atmosphere allowed adjustment of solvent partial pressure and enabled in conjunction with the thermal stage fine control of crucial parameters for structure formation, such as temperature and solvent drying kinetics. Neither spin coating nor blade coating are attractive film deposition techniques from an industrial point of view. Yet on the lab-scale, they offer repeatable and finely controllable deposition conditions. The further understanding of processing parameters may then be transferrable to roll-to-roll processing techniques such as slot-die coating or meniscus coating [20,21].

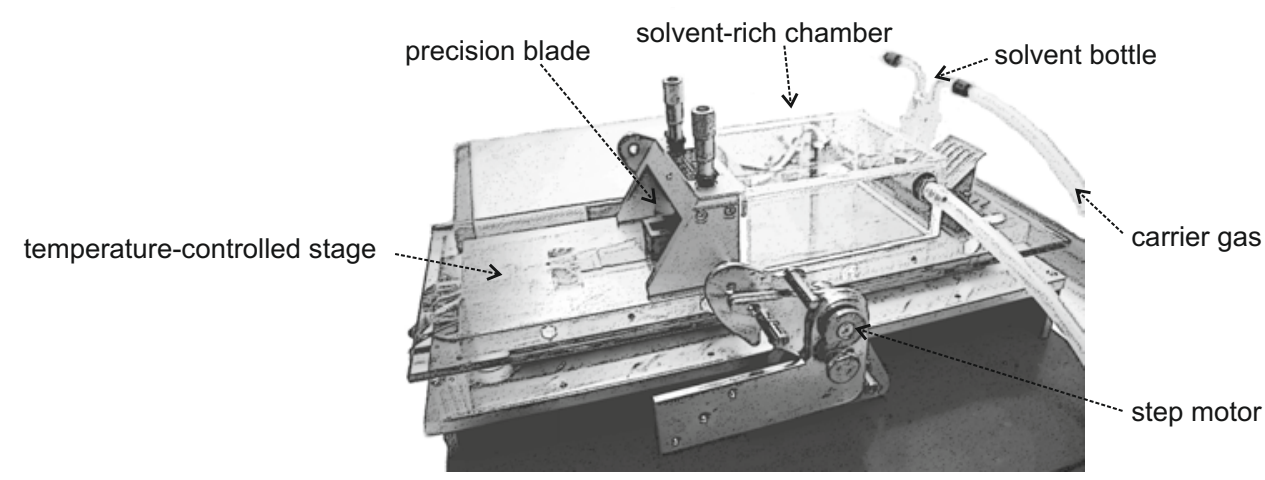

Figure 4.2: Blade coating in controlled environment. A commercial blade coating set-up was modified by the integration of a programmable heating element and an enclosed chamber, which is fed by solvent-rich atmosphere through a saturated carrier gas. 


\subsection{Materials characterisation}

A variety of characterisation techniques is necessary to understand and develop self-assembly driven material formation and functioning. Figure 4.3 shows an overview of the employed techniques in the course of this work and their accessible length scales. Their main operation principles are briefly explained in the following.

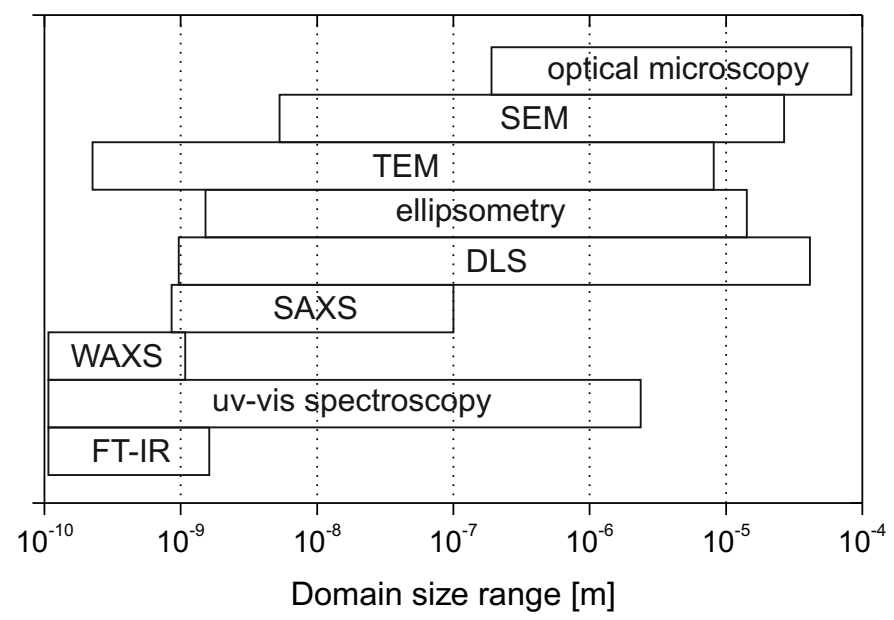

Figure 4.3: Overview of the employed characterisation techniques and their accessible length scales. Abbreviations from top to bottom: SEM - scanning electron microscopy; TEM - transmission electron microscopy; DLS - dynamic light scattering; SAXS - small angle X-ray scattering; WAXS - wide angle X-ray scattering; FT-IR - Fourier transform infrared spectroscopy. [22]

\subsubsection{Imaging techniques}

Various aspects of this work were concerned with structure formation on the sub-100 nanometre scale. The resolution of conventional visible-light microscopes is restricted to the spatial limit $s$ to which a beam of light can be focused, i.e. $s=\lambda / 2(n \sin \theta)$, where $n \sin \theta$ is the numerical aperture $N A<1.4$. In order to resolve smaller feature sizes a reduction of the employed wavelength is needed. Electron microscopes use a beam of electrons which is collimated and focused with electromagnetic lenses to illuminate the sample. Typical de Broglie wavelengths are in the range of $10^{-13}-10^{-11} \mathrm{~m}$. The study of electron-sample interactions therefore allows imaging with resolution down to the nanometre-length scale.

Possible interactions of an electron beam with a specimen are schematically shown in Figure 4.4. The variety of detectable signals opens up a multitude of ways to employ electron microscopy. 


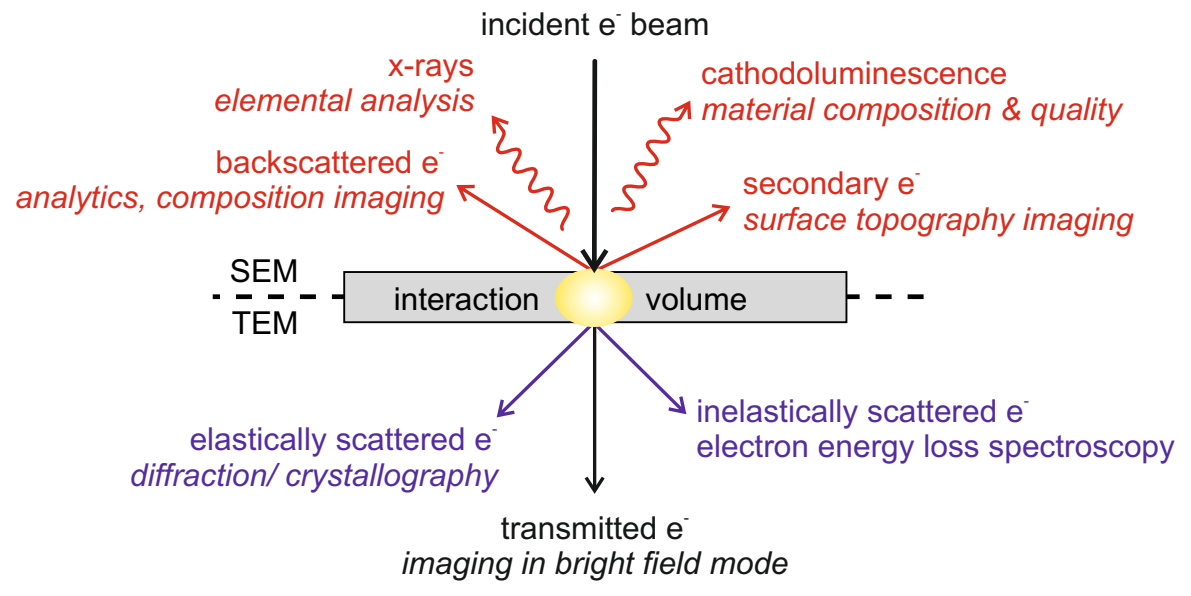

Figure 4.4: Overview of electron-matter interaction and corresponding use in electron microscopy. [23]

\section{Scanning electron microscopy}

In scanning electron microscopy (SEM) the electron beam is focused to a very small spot size and scanned over the surface of a sample. A number of different operational modes allow to gain insight information of the samples topography and material composition with up to $5 \mathrm{~nm}$ resolution in commercial instruments. The most common operational mode is the detection of secondary electrons, which can produce high resolution images of the samples surface. Secondary electrons are generated by inelastic scattering of primary electrons from atoms of the sample material. Due to their low energy $(<50 \mathrm{eV})$, only secondary electrons that are generated close to the surface $(<10 \mathrm{~nm})$ contribute to the signal, thereby providing high topographical contrast. In the most commonly used detection technique "In-Lens", secondary electrons which are generated directly in or near the spot centre are absorbed, re-accelerated and focused through an electromagnetic field to the "In-Lens" detector. A combination of scintillator and photomultipliers generate a signal that is translated into a pixel intensity. Imaging is achieved by scanning the electron beam over the sample

Further signal processing includes the detection of X-rays, backscattered electrons and cathodoluminescence. The detection of characteristic X-rays from the excitation of inner shell electrons is used in energy dispersive X-ray spectroscopy (EDX) to spatially resolve and carry out elemental analysis of the specimen. The detection of backscattered electrons offers further information on the elemental composition as the intensity of elastically scattered electrons is strongly dependent on the atomic number of the specimen. Spatially-resolved detection of cathodoluminescence corresponds to the collection of a sample's photoemission in the visible spectrum caused by the electron beam interaction. Energy decay after the excitation of outer shell electrons can take place via phonon or photon emission. The detection of intensity and 
spectrum of photoemission therefore delivers information on the composition, growth and quality of semiconductors and other materials.

In the course of this work SEM was carried out on a LEO Ultra 55 with a field emission source of typically $3 \mathrm{kV}$ electron acceleration voltage [24]. The instrument was mainly used for imaging of the samples topography via the "In-Lens" detection system. Occasionally EDX was employed for elemental characterisation of the specimen. In order to prevent charging effects a conductive platinum layer $(\sim 2 \mathrm{~nm})$ was deposited onto the sample with a sputter coater (Emitech K575X, 8s, $75 \mathrm{~mA}$ ). Typical SEM parameters were: working distance 3 $5 \mathrm{~mm}$, extractor voltage $5.9 \mathrm{~V}$, extractor current $162.8 \mu \mathrm{A}$, filament heating current $2.36 \mathrm{~A}$, gun vacuum $4.1 \times 10^{-10}$ mbar and system vacuum $5.8 \times 10^{-6}$ mbar.

\section{Transmission electron microscopy}

Transmission electron microscopy (TEM) is another technique that was employed for material characterisation. While sample preparation proves considerably more difficult and timeconsuming, the achievable image resolution is superior to SEM, typically with resolution below $1 \mathrm{~nm}$ in commercial instruments.

In the bright field imaging mode, only transmitted and weakly scattered electrons are collected due to the positioning of the objective aperture and focusing of the lens optics. Dark areas on the screen then correspond to strongly scattering regions in the sample. The resulting image of the sample can therefore reveal information on morphological features, structural composition and crystallinity. In diffraction mode the lens system is adjusted as such that the backfocal plane is focused. Electrons that leave the sample at identical angles undergo coherent scattering. The resulting Bragg reflections reveal information on the crystal structure, unit cell size, crystal orientation and the degree of polycrystallinity of the sample [23].

Cryo-ultramicrotomy allows the preparation of sufficiently thin hybrid or organic sample sections. This involves embedding of the sample in a viscous water-soluble compound, cooling to $-50{ }^{\circ} \mathrm{C}$ and sectioning in $30-40 \mathrm{~nm}$ slices using consecutively a glass and diamond knife on a piezoelectric-actuated motion stage. Alternatively ultrathin hybrid films can be floated onto a TEM grid using a sacrificial water-soluble underlayer ,e.g. poly (4-styrene sulfonic acid, to aid lift-off from the substrate [25].

Two different instruments were used in this work. During a research stay at Cornell University a FEI Tecnai T12 Spirit TEM (acceleration voltage $120 \mathrm{keV}$ ) was used under the supervision of Dr. Morgan Stefik. In Cambridge TEM imaging was carried out with Giorgio Divitini on a FEI Tecnai F20-G2 FEGTEM with a $200 \mathrm{kV}$ field emission gun. 


\subsubsection{Scattering techniques}

While real space imaging offers a direct route for the sampling of material arrangement, scattering techniques only allow structure determination in the reciprocal space. Results then have to be related to the real space by Fourier transformation. Scattering techniques are often complimentary to imaging as the obtainable results are statistically more reliable, since all scattering events in the beam passage contribute to the measured signal.

An ordered morphology, may it be an atomic crystal or a phase separated BCP structure, can be represented by a periodic lattice of scattering objects. Diffracted wavefronts undergo constructive interference if the Bragg condition is satisfied (see Figure 4.5a) [26]:

$$
n \lambda=2 d \sin \theta,
$$

where $n=1,2,3 \ldots$ is the order of peak, $\lambda$ is the wavelength of the incident beam, $d$ is the spacing of lattice planes in real space, and $\theta$ is the angle between the incident beam and the lattice plane. Periodicity in the sampling structure can therefore be investigated by variation of wavelength (Laue method) or scattering angles (Scherrer method, angular dependent scattering) in the appropriate range.

The construction of a reciprocal lattice proves useful to understand when the condition for constructive interference is met. The array of periodic scattering objects can be described with $\mathbf{R}=n_{1} \mathbf{a}_{1}+n_{2} \mathbf{a}_{2}+n_{3} \mathbf{a}_{3}$, where $n_{\mathrm{i}}$ are integers and $\mathbf{a}_{\mathrm{i}}$ are the primitive vectors that span the so-called Bravais lattice. For any translation of $\mathbf{R}\left(n_{1}, n_{2}, n_{3}\right)$ the lattice is therefore invariant. Incoming waves are generally described with $\Psi_{k}(\mathbf{r})=\Psi_{0} \exp ^{i \mathbf{r r}}$. Accordingly, the set of wavevectors $\mathbf{k}$ which result in plane waves with the periodicity of the Bravais lattice is defined by the condition:

$$
\Psi_{0} \exp ^{i \mathbf{k r}}=\Psi_{0} \exp ^{i \mathbf{k}(\mathbf{r}+\mathbf{R})}=\Psi_{0}\left(\exp ^{i \mathbf{k r}} \cdot \exp ^{i \mathbf{k} \mathbf{R}}\right)
$$

Therefore,

$$
\exp ^{i \mathbf{k R}}=1
$$

has to be fulfilled for all $\mathbf{R}$, leading to the definition of the reciprocal lattice vector in $\mathbf{k}$-space for which $\exp ^{i \mathbf{k R}}=\exp ^{i \mathbf{G R}}=1$, i.e. $\mathbf{G}=h \mathbf{b}_{1}+k \mathbf{b}_{2}+l \mathbf{b}_{3}$. Considering a set-up shown in Figure $4.5 \mathrm{~b}$, constructive interference of the diffracted beam is obtained for $\exp ^{i\left(\mathbf{k}_{\text {out }}-\mathbf{k}_{\text {in }}\right)}=1$. This determined the so-called Laue condition:

$$
\mathbf{q}=\frac{4 \pi \sin \theta}{\lambda}=\mathbf{k}_{\text {out }}-\mathbf{k}_{\text {in }}=\mathbf{G}(h, k, l) \text {. }
$$

Thus, for any scattering vector $\mathbf{q}=\mathbf{k}_{\text {out }}-\mathbf{k}_{\text {in }}$ that can be expressed with $\mathbf{G}(h, k, l)$, the Bragg 
condition is met for integer values of $h, k, l$. The three indices $h, k, l$ therefore define symmetry planes in the crystal lattice that result in characteristic reflection peaks. The related notation in real space $[h k l]$ refers to the direction $h \mathbf{a}_{1}+k \mathbf{a}_{2}+l \mathbf{a}_{3}$ of the normal vector for this characteristic set of crystal planes.

The above description does not consider the spatial dimensions of the scattering objects and the finite size of the crystal, which both affect the measured intensity $I(\mathbf{q})$. The spatial dimensions of the scattering object is generally described by a form factor $F(\mathbf{q})$ of the crystal's unit cell, while the finite size is accounted for by the convolution of the reciprocal lattice with a step function $\sigma(\mathbf{r})$ and the corresponding Fourier transform $\mathcal{F}[\sigma(\mathbf{r})]=\sum(\mathbf{q})$. The measured scattering intensity $I(\mathbf{q})$ therefore follows

$$
I(\mathbf{q}) \propto|F(q)|^{2} \cdot\left|Z(\mathbf{q}) * \sum(\mathbf{q})\right|^{2}
$$

where $Z$ (q defines the reciprocal lattice. This means that, in addition to the periodic arrangement, one can also obtain information on the overall size of the crystal and spatial dimensions of the lattice points from scattering experiments. See reference [27] for details.

The appropriate angular window for a fixed wavelength (typically $\lambda_{\mathrm{CuK} \alpha}=1.54 \AA$ ) then depends on the length scale of interest. For probing the atomic lattice of a solid state material, where $d_{\mathrm{hkl}}$ is comparable to the wavelength, wide-angle X-ray scattering with $2 \theta \sim 10^{\circ}-80^{\circ}$ allows to resolve the corresponding diffraction peaks. For feature sizes that are large compared to the wavelength of the beam, small angle X-ray scattering with $2 \theta \sim 0.1^{\circ}-5^{\circ}$ covers the correct angular range.
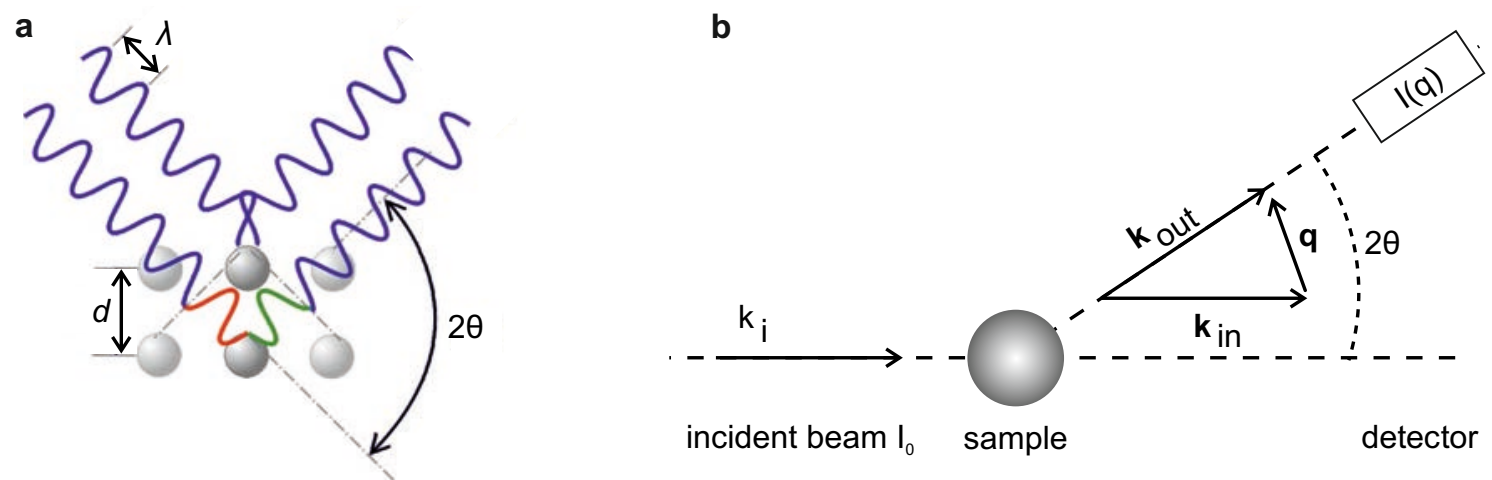

Figure 4.5: Principles of x-ray scattering techniques. a) Elastically scattered incident waves undergo constructive interference if the difference in path length $2 d \sin \theta$ is an integer multiple of the wavelength. b) Schematic of a scattering experiment. a): Adapted with permission from [28]. 


\section{Wide angle x-ray scattering}

Wide angle X-ray scattering (WAXS) was used in the course of this study to determine the crystallite size, crystal anisotropy and crystal phase mixing for materials such as $\mathrm{CaCO}_{3}, \mathrm{Fe}_{2} \mathrm{O}_{3}$, $\mathrm{SnO}, \mathrm{TiO}_{2}, \mathrm{ZnO}, \mathrm{WO}_{3}$ and others. Characterisation was carried out ex situ as well as in situ during the temperature treatment

All experiments were carried out in a $\theta-2 \theta$ configuration, i.e. the sample was kept fixed while source and detector were rotated by $\theta^{\circ}$ with respect to the sample plane. In situ WAXS was carried out on a Bruker "D8 Advance" X-ray diffractometer with integrated temperature stage. The actual temperature on the sample spot was calibrated via the lattice expansion of silicon and rutile $\mathrm{TiO}_{2}$. Unit cell parameters were calculated using the software "UnitCell" [29]. The temperature offset between thermocouple and sample spot was identified by comparing the lattice expansion with reference data [30, 31, 32]. Ex situ WAXS was carried out on a Bruker "D8" diffractometer with position sensitive detector ("LynxEye").

\section{Small angle $x$-ray scattering}

Small angle X-ray scattering (SAXS) is capable to detect elastic scattering events at lower scattering angles $\left(\sim 0.1^{\circ}-10^{\circ}\right)$ and allows therefore to obtain structural information on the mesoscopic length scale $(1-100 \mathrm{~nm})$. In a typical set-up a beam of monochromatic X-ray radiation is focused on a bulk sample. The scattered X-rays form a pattern, which is collected by a two-dimensional X-ray detector, situated at a long distance from the sample in order to achieve high spectral resolution at low scattering angles. In order to identify the material's spatial arrangement from the scattering pattern, the observed peak spacings have to be compared to characteristic sequences for model lattices of candidate morphologies. The corresponding sequence of diffraction peaks for model diblock copolymer morphologies can be found in Table 4.2. A typical diffraction pattern of a block copolymer / inorganic hybrid is shown in Figure 4.6, which is consistent with a hexagonal arrangement of cylinders.

Block copolymer phase morphologies are just one example for material structures that can be characterised by SAXS. In general, any one- to three-dimensional structural pattern can be resolved by sufficient electron contrast between the building blocks [33]. In contrast to typical diffraction patterns from WAXS of crystalline solids, peaks are typically broadened. This is a consequence of the long-range imperfection of self-assembled periodic mesostructures, giving rise to fluctuations in size and spacing of the scattering bodies [27].

A variety of SAXS instruments were used in the course of this work. Laboratory set-ups were employed in Cambridge (Bruker "Nanostar", Department of Materials Science) and at Cornell University (Rigaku "RU300" rotating anode, Department of Physics). For high flux 
experiments synchrotron radiation was utilised at the Cornell High Energy Synchrotron Source (CHESS, G1 beamline) and at the Diamond Light Source UK (I22 beamline). While SAXS experiments in Cambridge and at the Diamond light source were carried out by the author, characterisation at Cornell University was performed by Hiroaki Sai.

Table 4.2: Bragg spacings for model diblock copolymer morphologies [34].

\begin{tabular}{lrrrrr}
\hline Structure & \multicolumn{5}{c}{$q_{h k l} / q_{100}$} \\
\hline Lamellar & 1 & 2 & 3 & 4 & 5 \\
Hexagonal-packed cyl. & 1 & $\sqrt{3}$ & $\sqrt{4}$ & $\sqrt{7}$ & $\sqrt{9}$ \\
Gyroid & 1 & $\sqrt{4 / 3}$ & $\sqrt{7 / 3}$ & $\sqrt{8 / 3}$ & $\sqrt{10 / 3}$ \\
Body-centered cubic & 1 & $\sqrt{2}$ & $\sqrt{3}$ & $\sqrt{4}$ & $\sqrt{5}$ \\
Close-packed spherical & 1 & $\sqrt{4 / 3}$ & $\sqrt{8 / 3}$ & $\sqrt{11 / 3}$ & $\sqrt{12 / 3}$ \\
\hline
\end{tabular}
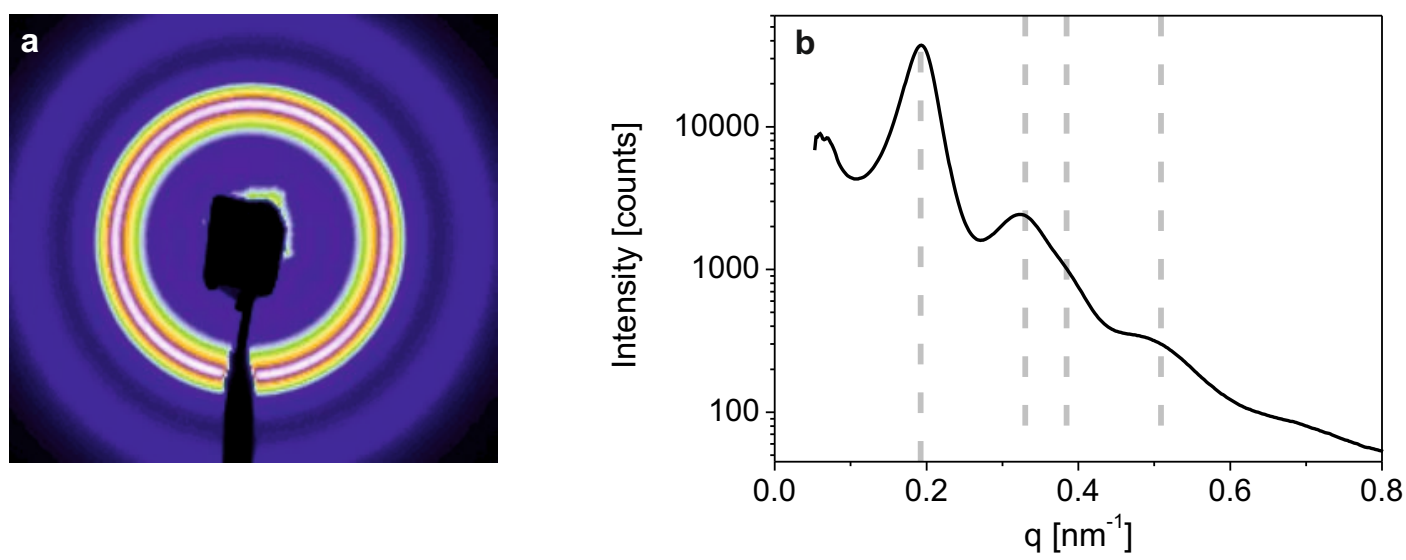

Figure 4.6: SAXS diffraction pattern of a BCP / inorganic hybrid. a) Example of a 2D SAXS image of a phase separated PI- $b$-PEO)/TiO 2 sample prepared in this study. b) Radially integrated SAXS data with expected peak positions $q_{h k l} / q_{100}=1, \sqrt{3}, \sqrt{4}, \sqrt{7}$ for an array of hexagonally packed cylinders.

\section{Dynamic light scattering}

In dynamic light scattering experiments (DLS), also known as photon correlation spectroscopy or quasi-elastic light scattering, temporal fluctuations in the speckle pattern of objects in solution are employed to yield their characteristic properties, such the particle size distribution, the Zeta potential or the molecular weight distribution of macromolecules.

The speed at which particles diffuse due to Brownian motion is measured by the intensity fluctuations of their speckle pattern. Comparing the intensity signal (I(t)) with the signal 
obtained at later times $(\mathrm{I}(\mathrm{t}+\tau), \mathrm{I}(\mathrm{t}+2 \tau), \mathrm{I}(\mathrm{t}+3 \tau))$ provides the contribution of slowly (large) and fast (small) diffusing objects in the sample volume. The correlation function $G(\tau)$ of the scattered intensity is therefore defined as

$$
G(\tau)=\frac{\langle I(t) I(t+\tau)\rangle}{\langle I(\tau)\rangle}
$$

with $\tau$ expressing the time difference of the correlation. For a large number of monodisperse particles, the correlation function is a single exponential decay function with the time delay $\tau$ :

$$
g_{1}(\tau)=A[1+B \exp (-2 \Gamma \tau)]
$$

where $A$ is the baseline of the correlation function and $B$ is the intercept. The decay rate $\Gamma$ is defined as

$$
\Gamma=D q^{2}
$$

where $D$ is the translational diffusion coefficient given by the Stokes - Einstein equation and

$$
q=\frac{4 \pi n}{\lambda_{0}} \sin \left(\frac{\theta}{2}\right)
$$

where $n$ is the refractive index of the dispersant, $\lambda_{0}$ is the wavelength of the laser and $\theta$ is the scattering angle. For polydisperse samples, Equation 4.10 can be written as

$$
G(\tau)=A\left[1+B g_{1}(\tau)^{2}\right]
$$

where $g_{1}(\tau)$ is the sum of all exponential functions that are contained in the correlation function. A comprehensive derivation of the DLS signal can be found in reference [35]. In Figure 4.7 a typical autocorrelation function $g_{1}(\tau)$ is plotted for a single exponential function of a single speckle.

The particle size distribution can then be obtained from the correlation function through a variety of algorithms. Usually a sum of multiple exponential curves is fitted to obtain the distribution of particle sizes, e.g. NNLS (non-negative least squares) or CONTIN. The obtained size distribution is by intensity. Using Mie and Rayleigh theory the sample intensity distribution can then be converted into a volume distribution. However, due to the fact that the intensity of light from elastic Rayleigh scattering scales as $I \alpha d^{6}$, conversion into volume fractions results in relatively high standard deviations, when particle diameters are much smaller than the laser wavelength [36]. 


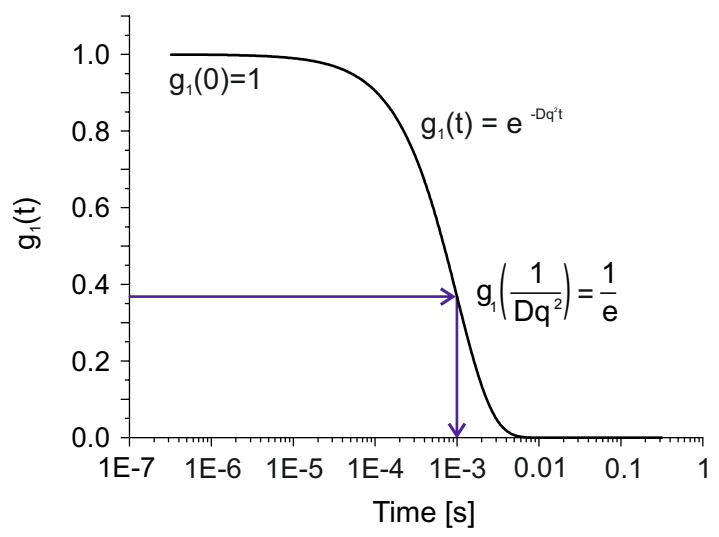

Figure 4.7: Ideal autocorrelation function $g_{1}(\tau)$, where a single exponential function can be used to fit the speckle intensity with time.

The Einstein-Stokes equation 4.15 describes the relation between the observed translational diffusion coefficient $D$ and the hydrodynamic diameter of the particle $d_{\text {iam, hyd }}$ :

$$
d_{\text {iam, hyd }}=\frac{k T}{6 \pi \eta D}
$$

where $T$ is the temperature, $k$ is the Boltzmann's constant, $\eta$ is the fluid viscosity, and $D$ is the translational diffusion coefficient. Other shapes may also be modelled [37].

Dynamic light scattering was employed in this study to measure particle size distributions of hydrolysed sol, synthesised nanocrystals or colloidal suspension between $2 \mathrm{~nm}$ and $1 \mu \mathrm{m}$. Experiments were carried out on a Malvern Zetasizer ZS instrument in backscattering mode (collection angle $173^{\circ}$ ) and adjustable penetration depth according to the scattering properties of the solution [38].

\subsubsection{Spectroscopy techniques}

\section{Ellipsometry}

Ellipsometry is a non-destructive technique to determine the refractive index, absorbance, thickness or optical anisotropy of a thin film. The technique bears its name from the underlying principle: the determination of a change in polarisation of initially elliptically polarised light upon reflection off a sample. The change in polarisation is represented by the so-called ellipsometric angles $\Psi$ and $\Delta$, which correspond to the amplitude ratio and phase difference upon reflection. These parameters can subsequently be related to the optical properties of the investigated film. The electric field components parallel $\left(E_{\mathrm{p}}\right)$ and perpendicular $\left(E_{\mathrm{s}}\right)$ to the plane of incidence are treated separately and can be described by the Fresnel reflection coefficients $r_{\mathrm{p}}=\left|r_{\mathrm{p}}\right| \exp ^{i \delta_{\mathrm{p}}}$ and $r_{\mathrm{s}}=\left|r_{\mathrm{s}}\right| \exp ^{i \delta_{\mathrm{s}}}$. For an experimental set-up shown in Figure 4.8, 
the detected and incident field components $E_{\text {out }}$ and $E_{\text {in }}$ can be described in form of so-called Jones matrices [39]:

$$
\left(\begin{array}{c}
E_{\mathrm{p}, \text { out }} \\
E_{\mathrm{s}, \text { out }}
\end{array}\right)=\left(\begin{array}{cc}
r_{\mathrm{pp}} & r_{\mathrm{sp}} \\
r_{\mathrm{ps}} & r_{\mathrm{ss}}
\end{array}\right) \cdot\left(\begin{array}{c}
E_{\mathrm{p}, \text { in }} \\
E_{\mathrm{s}, \text { in }}
\end{array}\right) .
$$

In a polariser - compensator - sample - analyser configuration, the Jones reflection matrix $\mathrm{R}$ is:

$$
\begin{aligned}
& R\left(\theta_{\mathrm{a}}\right)\left(\begin{array}{cc}
1 & 0 \\
0 & 0
\end{array}\right) R\left(-\theta_{\mathrm{a}}\right) \quad\left(\begin{array}{cc}
r_{\mathrm{p}} & 0 \\
0 & r_{\mathrm{s}}
\end{array}\right) R\left(\theta_{\mathrm{c}}\right)\left(\begin{array}{cc}
1 & 0 \\
0 & i
\end{array}\right) R\left(-\theta_{\mathrm{c}}\right) \quad R\left(\theta_{\mathrm{p}}\right)\left(\begin{array}{cc}
1 & 0 \\
0 & 0
\end{array}\right) R\left(-\theta_{\mathrm{p}}\right), \\
& \text { analyser (a) sample (s) compensator (c) polariser (p) }
\end{aligned}
$$

where $\theta_{\mathrm{a}}, \theta_{\mathrm{c}}$ and $\theta_{\mathrm{p}}$ are the angles of analyser, compensator and polariser with respect to the plane of incidence, and $R\left( \pm \theta_{\mathrm{a}, \mathrm{c}, \mathrm{p}}\right)$ are the rotation matrices:

$$
R\left( \pm \theta_{\mathrm{a}, \mathrm{c}, \mathrm{p}}\right)=\left(\begin{array}{cc}
\cos \theta_{\mathrm{a}, \mathrm{c}, \mathrm{p}} & \pm \sin \theta_{\mathrm{a}, \mathrm{c}, \mathrm{p}} \\
\mp \sin \theta_{\mathrm{a}, \mathrm{c}, \mathrm{p}} & \cos \theta_{\mathrm{a}, \mathrm{c}, \mathrm{p}}
\end{array}\right)
$$

The ellipsometric angles $\Psi$ and $\Delta$ then follow with

$$
\begin{aligned}
& \tan \Psi=\frac{\left|r_{\mathrm{p}}\right|}{\left|r_{\mathrm{s}}\right|} \\
& \Delta=\delta_{\mathrm{p}}-\delta_{\mathrm{s}} .
\end{aligned}
$$

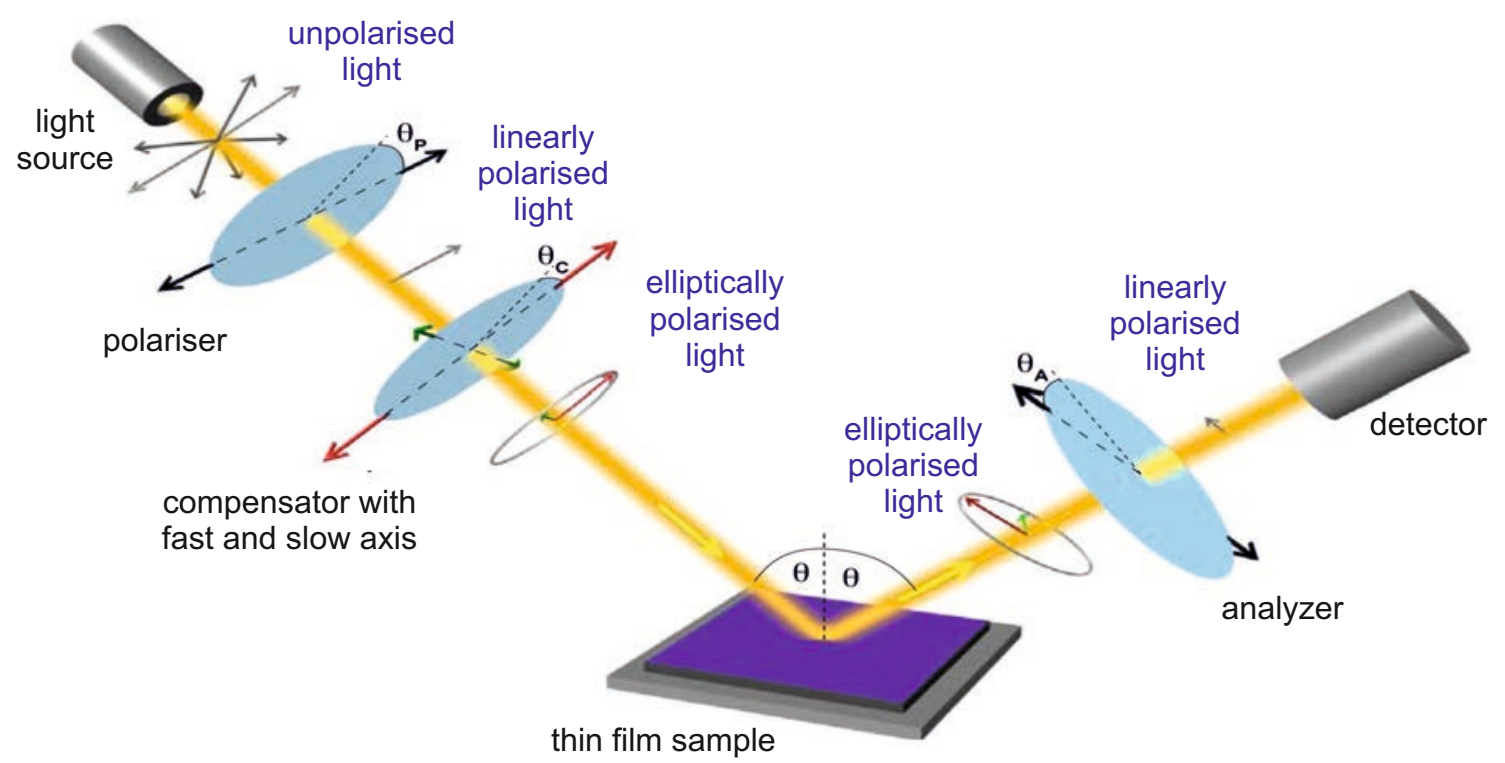

Figure 4.8: Experimental set-up for ellipsometric thin film characterisation. This configuration is known as p-c-s-a: polariser - compensator - sample - analyser. Adapted with permission from [25]. Copyright 2011 Springer Science+Business Media. 
Ellipsometry of optical films is an indirect technique, i.e. access to the optical constants is only possible by regression analysis with a model that includes known parameters and reasonable estimates of the fitting variables. The quality of the iteratively obtained best fit can be evaluated by a mean free error value (MSE), which is a measure of the difference between the measured and modelled optical response.

In this work two different techniques were used, imaging ellipsometry and spectroscopic ellipsometry. Imaging ellipsometry was carried out on a commercial instrument (Nanofilm "ep3se"). The built-in imaging set-up, consisting of a 5x magnifying lens and a spatially resolving CCD camera, allows to choose an area of interest. The polarisation change by the sample is then determined by nulling ellipsometry. This technique involves the rotation of polariser and analyser to find the minimum detection signal at a fixed angle of the compensator. The "nulling condition" therefore corresponds to a position, where the light reflected off the sample is linearly polarised and subsequently blocked off by the analyser, set at $90^{\circ}$ with respect to the axis of linear polarisation. In order to improve the fitting quality, measurements are repeated for a range of angles of incidence $\theta$. While imaging ellipsometry operated with nulling condition allows to determine the optical constants with high accuracy, this is limited to a fixed wavelength of incident light. In order to obtain spectroscopic values for refractive index and absorption, a spectroscopic ellipsometer was used (J.A. Wollam "alpha-SE", spectral range $380-900 \mathrm{~nm}$ ). This instrument does not operate using a nulling principle (which would be too time consuming) but only has a rotating compensator positioned behind the sample. The element turns in discrete steps and records at every position the intensity of all wavelength channels [40].

\section{Microspectroscopy}

Microspectroscopy is essentially the quantitative study of light-matter interactions with the spatial resolution of a visible light microscope. This is realised by coupling a spectrometer to the microscope's beam path, as illustrated in Figure 4.9. The microspectroscopy set-up used in this study, consisted of an Olympus BX-51 microscope and an Ocean Optics QE 65000 spectrometer. By default the build-in halogen lamp of the microscope with a spectral range of $\sim 380-860 \mathrm{~nm}$ was employed as light source but alternative sources of wider spectral distribution (e.g. a combined deuterium tungsten halogen with a spectral range of 215 - $2000 \mathrm{~nm}$, Ocean Optics "DH-2000”) or a narrow line-width laser can be coupled in. A computer-controlled x-y-translational stage (Prior Scientific "ProScan II") allows the movement of the sample with sub- $\mu \mathrm{m}$ accuracy. The use of magnifying lenses imply a certain angular spread in the wave vector distribution, which is determined by the numerical aperture 
of the lens. In order to keep the wave vector dispersion to a minimum, most experiments were carried out with a $5 x$ magnifying objective ( $N A=0.15$, Olympus "UIS2 MPlanFLN"). With fixed magnification, the spot size of detection depends on the core dimensions of the optical fibre, acting as a pinhole in the conjugate to the focal plane. Using fibre diameters between $50 \mu \mathrm{m}$ and $1000 \mu \mathrm{m}$, the collection spot size therefore be varied between $15 \mu \mathrm{m}$ and $300 \mu \mathrm{m}$.

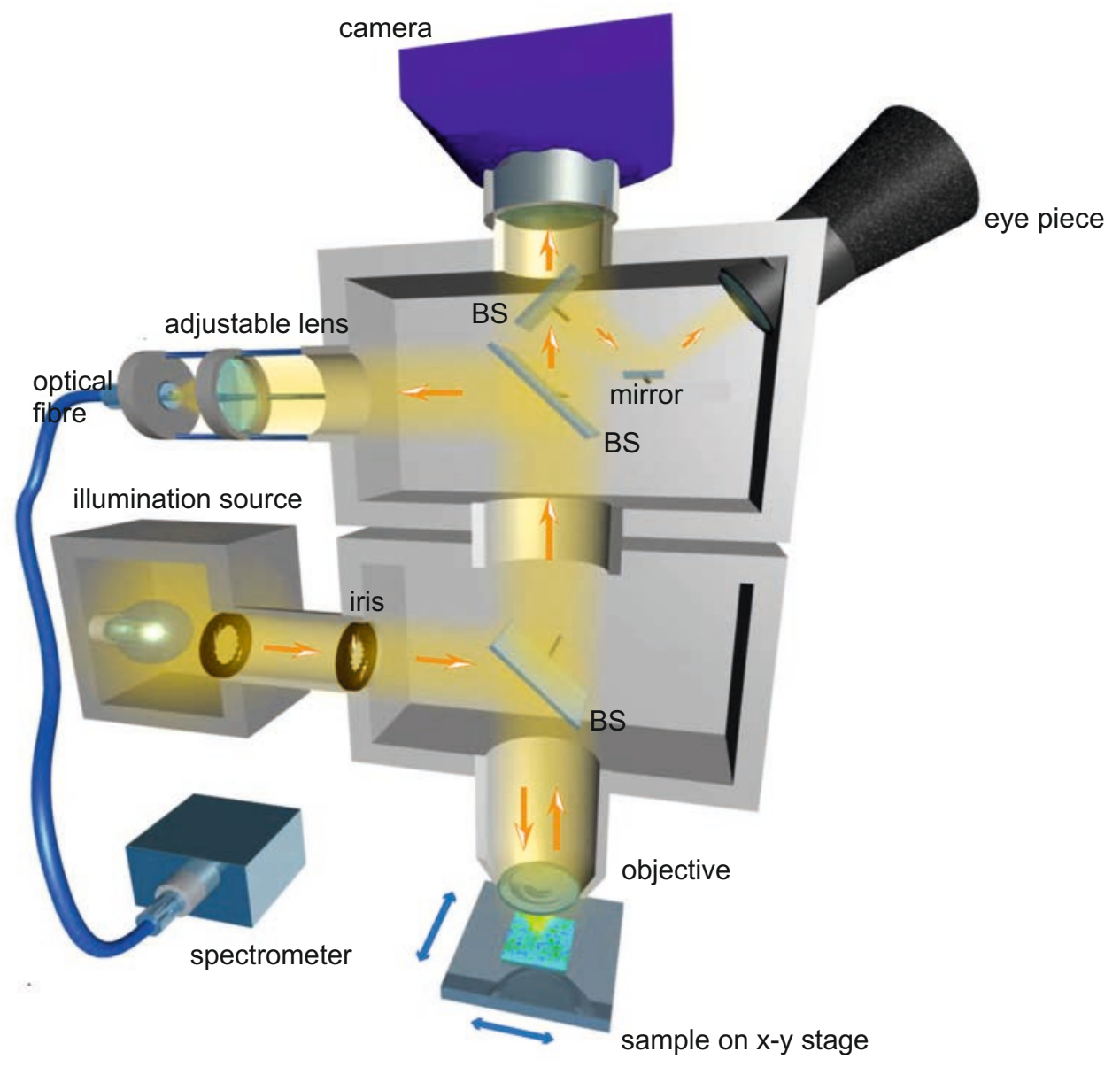

Figure 4.9: Microspectroscopy set-up. The conventional set-up of a light microscope is extended by the addition of a spectroscopy compartment, which is connected via a beamsplitter (BS) to the instrument's main beam path. Sampling of the spectrometer is carried out via an optical fibre that is mounted in the conjugate of the focal plane and acts as a pinhole. Adapted with permission from [25]. Copyright 2011 Springer Science+Business Media.

\section{Fourier transform infrared spectroscopy}

Fourier transform infrared spectroscopy (FT-IR) is a technique to investigate the molecular fingerprint of a sample by measuring its absorption in the infrared frequency range. Sampling is typically carried out in a range of $\lambda \sim 0.78-1000 \mu \mathrm{m}$, which corresponds to a wavenumber of $\tilde{v}=1 / \lambda \sim 13000-10 \mathrm{~cm}^{-1}$. FT-IR therefore allows to quantify transitions between quantised 
vibrational energy states. In a non-linear molecule the number of possible vibrational modes is $3 N-6$, where $N$ is the number of atoms in the molecule. Six degrees of freedom are determined by the collective translational and rotational motion of the molecule. The energy difference between the ground state and the first excited state of most vibrational modes is typically in the mid-infrared $\left(\sim 4000-400 \mathrm{~cm}^{-1}\right)$. Many vibrational modes only have large displacements for a specific hot spot, while the rest of the molecule has no or little influence. These modes allow the determination of specific functional groups and enable the classification of the molecule [41].

FTIR instruments are typically based on a Michelson-interferometer, which encodes an interference pattern by the addition of a modulated optical path difference of one-half to the IR-beam. The measurement of absorption via an interferogram allows to sample a multitude of frequencies simultaneously. Transformation into a frequency spectrum is then accomplished via Fourier transformation [42].

In this work FTIR was carried out on a commercially available instrument (Thermo Scientific "Nicolet iS10"). This allowed to monitor the decomposition of marker molecules under photocatalytic decomposition. The investigated molecule, stearic acid, showed three characteristic features in the observed range that enabled the quantitative determination in the sample volume: the asymmetric in-plane $\mathrm{CH}$ stretching mode of the $\mathrm{CH}_{3}$ group $\left(\tilde{v} \sim 2958 \mathrm{~cm}^{-1}\right)$ as well as the symmetric $\left(\tilde{v} \sim 2923 \mathrm{~cm}^{-1}\right)$ and asymmetric $\left(\tilde{v} \sim 2853 \mathrm{~cm}^{-1}\right) \mathrm{CH}$ stretching modes of the $\mathrm{CH}_{2}$ group.

\section{Bibliography}

[1] S. Bagshaw, E. Prouzet, and T. Pinnavaia, "Templating of mesoporous molecular-sieves by nonionic polyethylene oxide surfactants," Science, vol. 269, no. 5228, pp. 1242-1244, 1995.

[2] G. Floudas, R. Ulrich, and U. Wiesner, "Microphase separation in poly(isoprene-bethylene oxide) diblock copolymer melts. I. Phase state and kinetics of the order-to-order transitions," Journal Of Chemical Physics, vol. 110, no. 1, pp. 652-663, 1999.

[3] M. Templin, A. Franck, A. DuChesne, H. Leist, Y. Zhang, R. Ulrich, V. Schädler, and U. Wiesner, "Organically modified aluminosilicate mesostructures from block copolymer phases," Science, vol. 278, no. 5344, pp. 1795-1798, 1997.

[4] S. Renker, S. Mahajan, D. Babski, I. Schnell, A. Jain, J. Gutmann, Y. Zhang, S. Gruner, H. Spiess, and U. Wiesner, "Nanostructure and shape control in polymer-ceramic hybrids from poly(ethylene oxide)-block-poly(hexyl methacrylate) and aluminosilicates derived from them," Macromolecular Chemistry and Physics, vol. 205, no. 8, pp. 1021-1030, 2004. 
[5] J. Allgaier, A. Poppe, L. Willner, and D. Richter, "Synthesis and characterization of poly[1,4-isoprene-b-(ethylene oxide)] and poly[ethylene-co-propylene-b-(ethylene oxide)] block copolymers," Macromolecules, vol. 30, no. 6, pp. 1582-1586, 1997.

[6] G. Floudas, B. Vazaiou, F. Schipper, R. Ulrich, U. Wiesner, H. Iatrou, and N. Hadjichristidis, "Poly(ethylene oxide-b-isoprene) diblock copolymer phase diagram," Macromolecules, vol. 34, no. 9, pp. 2947-2957, 2001.

[7] C. Brinker and G. Scherer, Sol-Gel Science: The Physics and Chemistry of Sol-Gel Processing. Academic Press, 1st ed., 1990.

[8] S. Warren, F. Disalvo, and U. Wiesner, "Nanoparticle-tuned assembly and disassembly of mesostructured silica hybrids," Nature Materials, vol. 6, no. 2, pp. 156-U23, 2007.

[9] A. Jain and U. Wiesner, "Silica-type mesostructures from block copolymer phases: formation mechanism and generalization to the dense nanoparticle regime," Macromolecules, vol. 37, no. 15, pp. 5665-5670, 2004.

[10] S. Boettcher, J. Fan, C.-K. Tsung, Q. Shi, and G. Stucky, "Harnessing the sol-gel process for the assembly of non-silicate mesostructured oxide materials," Accounts Of Chemical Research, vol. 40, no. 9, pp. 784-792, 2007.

[11] C. Sanchez, C. Boissière, D. Grosso, C. Laberty, and L. Nicole, "Design, synthesis, and properties of inorganic and hybrid thin films having periodically organized nanoporosity," Chemistry Of Materials, vol. 20, no. 3, pp. 682-737, 2008.

[12] H. Schmidt and H. Wolter, "Organically modified ceramics and their applications," Journal Of Non-Crystalline Solids, vol. 121, no. 1-3, pp. 428-435, 1990.

[13] S. Warren, Nanoparticle-block copolymer self-assembly. PhD thesis, Cornell University, Faculty of the Graduate School, 2007.

[14] S. De Paul, J. Zwanziger, R. Ulrich, U. Wiesner, and H. Spiess, "Structure, mobility, and interface characterization of self-organized organic-inorganic hybrid materials by solidstate NMR," Journal Of The American Chemical Society, vol. 121, no. 24, pp. 57275736, 1999.

[15] P. Mokarian-Tabari, M. Geoghegan, J. R. Howse, S. Y. Heriot, R. Thompson, and R. Jones, "Quantitative evaluation of evaporation rate during spin-coating of polymer blend films: control of film structure through defined-atmosphere solvent-casting," $\mathrm{Eu}$ ropean Physical Journal E, vol. 33, no. 4, pp. 283-289, 2010.

[16] C. Lawrence, "The mechanics of spin coating of polymer films," Physics Of Fluids, vol. 31, no. 10, pp. 2786-2795, 1988.

[17] D. Schubert and T. Dunkel, "Spin coating from a molecular point of view: its concentration regimes, influence of molar mass and distribution," Materials Research Innovations, vol. 7, no. 5, pp. 314-321, 2003.

[18] D. Schubert, "Spin coating as a method for polymer molecular weight determination," Polymer Bulletin, vol. 38, no. 2, pp. 177-184, 1997.

[19] M. Kolle, personal graphics collection 
[20] F. Krebs, "Fabrication and processing of polymer solar cells: a review of printing and coating techniques," Solar Energy Materials And Solar Cells, vol. 93, no. 4, pp. 394$412,2009$.

[21] S. Weinstein and K. Ruschak, "Coating flows," Annual Review Of Fluid Mechanics, vol. 36, pp. 29-53, 2004.

[22] U. Gedde, Polymer physics. Kluwer Academic Publishers, 1st ed., 1995.

[23] D. Williams and C. Carter, Transmission electron microscopy: a textbook for materials science. Springer, 2009.

[24] J. Ackermann, "Manual for the SUPRA and ULTRA scanning electron microscopes." Zeiss SMT 1td., Manual SmartSEM V5.00, 2005.

[25] M. Kolle, Photonic structures inspired by nature. $\mathrm{PhD}$ thesis, University of Cambridge, Physics Department, 2010.

[26] W. Bragg, "The diffraction of short electromagnetic waves by a crystal," Proceedings of the Cambridge Philosophical Society, vol. 17, pp. 43-57, 1913.

[27] R.-J. Roe, Methods of x-ray and neutron scattering in polymer science. Oxford University Press, 2000.

[28] Wikimedia - public domain graphic http://commons.wikimedia.org, 2011.

[29] T. Holland and S. Redfern, "Unit cell refinement from powder diffraction data: The use of regression diagnostics," Mineralogical Magazine, vol. 61, no. 1, pp. 65-77, 1997.

[30] W. Yim and R. Paff, "Thermal-expansion of AlN, sapphire, and silicon," Journal Of Applied Physics, vol. 45, no. 3, pp. 1456-1457, 1974.

[31] E. Meagher and L. G.A., "Polyhedral thermal expansion in the $\mathrm{TiO}_{2}$ polymorphs: refinement of the crystal structures of rutile and brookite at high temperatures," Canadian Mineralogist, vol. 17, pp. 77-85, 1979.

[32] U. Diebold, "The surface science of titanium dioxide," Surface Science Reports, vol. 48, no. 5-8, pp. 53-229, 2003.

[33] F. Zernike and J. Prins, "Die Beugung von Röntgenstrahlen in Flüssigkeiten als Effekt der Molekularordnung," Zeitschrift für Physik, vol. 41, pp. 184-194, 1926.

[34] I. Hamley and V. Castelletto, "Small-angle scattering of block copolymers in the melt, solution and crystal states," Progress In Polymer Science, vol. 29, no. 9, pp. 909-948, 2004.

[35] K. Schätzel, "Single-photon correlation techniques," in Dynamic light scattering, the method and some applications, Oxford University Press, 1993.

[36] Malvern Instruments, "Dynamic light scattering: an introduction in 30 minutes." MRK656-01, 2001.

[37] B. Berne and R. Pecora, Dynamic Light Scattering - with applications to chemistry, biology, and physics. Dover Publications Inc., 2000.

[38] Malvern Instruments, “Zetasizer nano series user manual.” MAN0317, 2004. 
[39] R. Jones, "A new calculus for the treatment of optical systems I. Description and discussion of the calculus," Journal Of The Optical Society Of America, vol. 31, no. 7, pp. 488-493, 1941.

[40] J. Lee, P. Rovira, I. An, and R. Collins, "Rotating-compensator multichannel ellipsometry: applications for real time Stokes vector spectroscopy of thin film growth," Review Of Scientific Instruments, vol. 69, no. 4, pp. 1800-1810, 1998.

[41] P. Griffiths and J. de Haseth, Fourier transform infrared spectroscopy. John Wiley \& Sons, 2nd ed., 2007.

[42] C.-P. Sherman Hsu, Infrared spectroscopy in handbook of instrumental techniques for anylytical chemistry. Prentice Hall, 1997. 



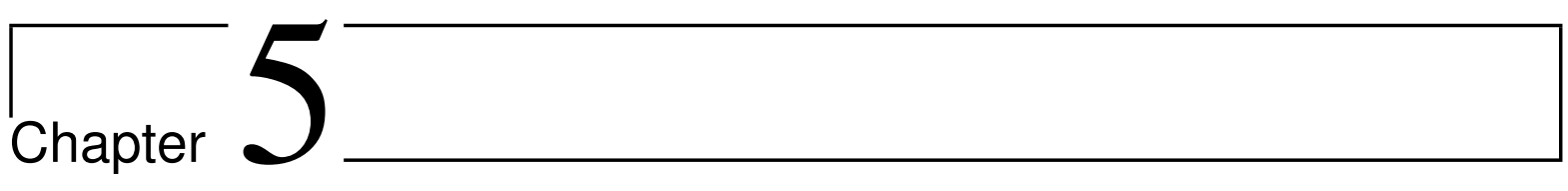

\section{Block copolymer-induced structure control for inorganic nanomaterials}

\subsection{Introduction}

The conditions for structure-control in a co-assembly approach using block-copolymers (BCPs) as structure-directing host and an inorganic sol as guest material have been discussed in Section 1.2.3. Four requirements have to be fulfilled in order to reach equilibrium morphologies: (1) the guest material needs to exhibit selective interaction with one block of the polymer, (2) the inorganic material has to disperse into small units in comparison to the length scale of the polymer chain, (3) the kinetics of the gelation process need to be slow in comparison to the time duration of the polymer phase separation, and (4) a common solvent system has to dissolve all components and enable even and sufficiently slow evaporation of the volatile species.

Condition one can easily be met. Most polymer structure-directing agents (SDAs) have a hydrophilic poly(ethylene oxide) (PEO) block, which interacts with a polar inorganic guest by forming hydrogen bonds $[1,2]$. Various strategies can be employed to tune the reactivity of the inorganic sol-gel transformation in order to meet condition number two. Typically, hydrolysed sols is stabilised in form of $<4 \mathrm{~nm}$ sized colloids to allow complexation with the PEO chain. In contrast, condition number three and four still pose a major challenge for structure control of certain materials, which shall be discussed in the following.

The SDA driven co-assembly of silica has led to remarkable results over the years, indicating that all four conditions can be fulfilled in this materials system for the assembly of bulk materials. For PI- $b$-PEO in combination with a silica-type sol, all equilibrium morphologies of a diblock copolymer phase diagram have been reported, including inorganic-rich spheres, 
hexagonally arranged cylinders, gyroids, lamellae and the corresponding inverse arrangements of hydrophobic and hydrophilic components [3, 4]. For the most widely studied materials couple of PEO- $b$-PPO- $b$-PEO (Pluronic) in conjunction with sol-gel-derived silica, equilibrium morphologies have been realised where the inorganic materials resides in the majority component of lamellae, hexagonal cylinders, gyroids and spherical arrangements $[5,6]$.

The general applicability of the SDA approach is mostly limited by two aspects. For many applications, such as in batteries, supercapacitors, dye-sensitised solar cells, fuel cells and electrochromic devices, the morphology has to be fabricated in a thin film, where the evaporation kinetics of the solvent greatly complicates the equilibration of the phase morphology [7]. Further challenges emerge when extending the SDA approach from silica to other materials, such as transition metal oxides. For $\mathrm{TiO}_{2}$, high chemical reactivity and multiple stable coordination states of the titanium precursor greatly limit the control over the kinetics of the sol-gel reaction. As an example, the hydrolysis of titanium alkoxide $\mathrm{Ti}(\mathrm{OR})_{4}$ is $10^{5}$ times faster than that of the silica counterpart $\mathrm{Si}(\mathrm{OR})_{4}$ [8]. Routes to decelerate the gelation of the inorganic sol include the addition of stabilising ligands, reacting under acidic conditions or non-hydrolytic synthesis. Usually a significant amount of aqueous $\mathrm{HCl}$ is added in order to obtain a stable sol for BCP-directed material assembly [5]. This, however, results in an oversupply of water (typically four times as much as needed for hydrolysis), which consequently resides in the hydrophilic block and impedes the simultaneous evaporation of volatile components from both blocks in a non-aqueous solvent mixture.

Table 5.1 presents an overview of commonly employed SDAs. The widely-used BCP Pluronic exhibits a much lower Flory-Huggins interaction parameter $\chi$ in comparison to the PHB- $b$-PEO and the PI- $b$-PEO systems. This means that polymer phase separation occurs on significantly slower time scales, which is the reason why most successful material routes towards equilibrium morphologies involve daylong preparation under controlled humidity and temperature. As an example, high order in Pluronic-directed thin films with gyroid morphology was achieved by aging of the precursor solution for 10 days, followed by annealing of the coated film for 12 hours after deposition [9]. In contrast, morphologies in PI- $b$-PEO-directed approaches typically form within minutes [10].

A high $\chi$ interaction parameter enhances phase separation with long range order and allows faster processing as well as lower sensitivity to the preparation conditions. This comes, however, at the expense of higher dissimilarity in the solubility of the components. When using the BCP Pluronic, water and ethanol are good solvents for both polymer blocks and the inorganic material. In contrast, PHB and PI are not water-soluble, and overall solubility in aqueous solutions can only be achieved by limiting the volume fraction of these hydrophobic components [15]. Non-hydrolytic sol-gel chemistry and the use of polar solvents may in principle be one 
Table 5.1: Comparison of structure-directing agents for ordered mesoporous silicates [11].

\begin{tabular}{cccc}
\hline & $\begin{array}{c}\text { PEO- } b \text {-PPO- } b \text {-PEO } \\
\text { (Pluronic) }\end{array}$ & $\begin{array}{c}\text { PHB- } b \text {-PEO } \\
(\text { KLE })\end{array}$ & PI- $b$-PEO \\
\hline \hline Flory Huggins $\chi$ at $50{ }^{\circ} \mathrm{C}$ & 0.085 & $\sim 0.33$ & 0.33 \\
$M_{\mathrm{n}}(\mathrm{kg} / \mathrm{mol})$ & $2-13$ & $8-10$ & $10-84$ \\
wt\% PEO & $30-70 \%$ & $37-57 \%$ & $8-38 \%$ \\
Solubility in water / alhohol & yes & limited & limited \\
Hybrid morphologies & $\mathrm{CM}$, iHEX, G, $\mathrm{L}$ & $\mathrm{CM}$ & (i)CM, (i)HEX, PN, G, L \\
Pore size range $(\mathrm{nm})$ & $5-10 \mathrm{~nm}$ & $12-13$ & $11-50$ \\
References & {$[5,6,12,13,14]$} & {$[15]$} & {$[4,16,17]$} \\
\hline
\end{tabular}

i: inverse; CM: close-packed micelles; HEX: hexagonally arranged cylinders

G: gyroid; L: lamellae; PN: plumbers nightmare

route to overcome these solubility issues but control over the non-hydrolytic reaction pathways proves difficult, especially for $\mathrm{TiO}_{2}[18,19]$.

This chapter presents strategies to overcome the outlined limitations in order to fully exploit the potential of the PI- $b$-PEO SDA system for morphology control in the bulk and in thin films.

\subsection{Experimental}

Three different BCPs were used as structure-directing agents: I) PI- $b$-PEO with $M_{\mathrm{n}, \mathrm{tot}}=$ $34.4 \mathrm{~kg} / \mathrm{mol}$ and $28.4 \mathrm{vol} \%$ PEO, II) PI- $b$-PEO with $M_{\mathrm{n}, \text { tot }}=91.6 \mathrm{~kg} / \mathrm{mol}$ and $31.2 \mathrm{vol} \%$ PEO and III) PI- $b$-PS- $b$-PEO with $M_{\mathrm{n}, \mathrm{tot}}=53.4 \mathrm{~kg} / \mathrm{mol}$ and 29.4 vol\% PEO. See Table 4.1 for details. The polymer was weighed and subsequently dissolved in a solvent system of choice, such as tetrahydrofuran (THF, $99.97 \%$ ), an azeotrope solvent mixture of $72.84 \mathrm{wt} \%$ toluene $(99.8 \%)$ and $27.16 \mathrm{wt} \%$ 1-butanol (99\%) or anisole (anhydrous, $99.7 \%)$.

\section{Preparation of $\mathrm{TiO}_{2}$ morphologies in the bulk and in thin films}

Titanium containing sol was synthesised by quickly adding $0.69 \mathrm{ml}$ of $\mathrm{HCl}(37 \%)$ into $1 \mathrm{ml}$ of titanium ethoxide (purum) under vigorous stirring at ambient conditions. Depending on the inorganic to organic weight ratio, the adequate amount of sol was subsequently mixed with PI- $b$-PEO copolymer in $7 \mathrm{ml}$ THF $(99.97 \%)$. For a weight ratio of 1:1 between the resulting $\mathrm{TiO}_{2}$ after fabrication and the polymer in initial solution, $0.44 \mathrm{ml}$ sol was added to $0.1 \mathrm{~g} \mathrm{BCP}$. After solvent evaporation in a Petri dish at $50{ }^{\circ} \mathrm{C}$, the hybrid material was redissolved in an azeotrope solvent mixture of toluene (apolar, 99.8\%) and 1-butanol (polar, 99\%) and then either processed by solvent casting in a covered Petri dish at $50{ }^{\circ} \mathrm{C}$ to obtain bulk material or 
spin coated for thin film processing. The dry hybrid material was subsequently annealed by slow ramping to $130^{\circ} \mathrm{C}$ (180 min linear ramp, $30 \mathrm{~min}$ dwell). Where indicated, calcination was carried out at $500{ }^{\circ} \mathrm{C}$ (90 min ramp, 2 hour dwell).

\section{Preparation of silica-type thin films}

Aluminosilicate sol was prepared as follows: $2.8 \mathrm{~g}$ (3-glycidyloxypropyl)trimethoxysilane ( $\geq$ $98 \%$ ) and $0.32 \mathrm{~g}$ aluminum-tri-sec-butoxide (97\%) were mixed with $20 \mathrm{mg} \mathrm{KCl}(99.9995 \%)$ and promptly placed into an ice bath. In a first hydrolysis step, $0.135 \mathrm{ml}$ of $10 \mathrm{mM} \mathrm{HCl}$ was added dropwise in $5 \mathrm{~s}$ intervals at $0^{\circ} \mathrm{C}$ and stirred for $15 \mathrm{~min}$. After warming the solution to room temperature, $0.85 \mathrm{ml}$ aqueous $\mathrm{HCl}(10 \mathrm{mM})$ was further added dropwise. The sol was then stirred for $20 \mathrm{~min}$ before adding to the polymer solution in a mixing ratio, which was defined by the weight of the resulting aluminosilicate compared to the weight of polymer in the initial solution. After subsequent stirring for another $60 \mathrm{~min}$, the solution was processed by spin coating or evaporation-controlled blade coating. Ensuing solvent evaporation, the hybrid films were annealed on a hotplate by slowly increasing the temperature to $200^{\circ} \mathrm{C}(180 \mathrm{~min}$ linear ramp, 30 min dwell). In a final step, the organic component of the hybrid films was removed by reactive ion etching in oxygen plasma $(30 \mathrm{~min}, 100 \mathrm{~W}, 0.33 \mathrm{mbar}$, STS Instruments, 320PC RIE).

\subsection{Results and discussion}

\subsubsection{Block copolymer induced structure control in hybrid bulk assem- blies}

As outlined in the introductory section, the use of hydrolytic sol-gel chemistry poses an inherent challenge to the use of PI- $b$-PEO with a majority block of the hydrophobic PI. A typical approach for the synthesis of anatase $\mathrm{TiO}_{2}$ therefore consists of separately preparing a hydrolytic sol containing the titanium precursor and a solution of the BCP in THF. The two solutions are subsequently mixed before the solvents are evaporated. The resulting morphology of a self-assembled hybrid material is shown in the transmission electron microscopy (TEM) images in Figure 5.1a. For all bulk samples shown in this section, specimens were prepared by ultra-microtomy to allow imaging in transmission mode.

The one-pot procedure, where the mixed solution is slowly allowed to evaporate in a Petri dish at $50{ }^{\circ} \mathrm{C}$ under a glass dome, results in a micellar arrangement. This is somewhat similar to reported morphologies for the PEO- $b$-PHB SDA system [15]. The reason for micellar 
arrangement may be multifold. For a mixture of $5 \mathrm{wt} \%$ water and $95 \mathrm{wt} \% \mathrm{THF}$, the solvents should form an azeotrope with a common boiling point of $66^{\circ} \mathrm{C}$ [20]. The low solubility of the PI polymer block in a water-rich solvent mixture at the later stages of solvent evaporation may therefore not be the origin for the micellisation of the system. Another reason may be associated with the fast evaporation of THF, one of the rare solvents that dissolves all components. This can result in quenching of the phase morphology by the $\mathrm{TiO}_{2}$ condensation reaction before the equilibrium morphology is reached. In order to overcome micellisation, an additional step in the fabrication procedure was introduced in form of a solvent exchange. The initial mixture was slowly dried in a Petri dish and subsequently redissolved in a blend of 1-butanol and toluene, which was mixed in a volume ratio that allowed the formation of an azeotrope [21]. The azeotrope exhibits a boiling point of $105^{\circ} \mathrm{C}$ and should enable good solubility of hydrophilic and hydrophobic components. The resulting morphology after the subsequent second evaporation process is shown in the TEM image in Figure 5.1c. Here, the $\mathrm{TiO}_{2}$ is arranged in an inverse hexagonal matrix, which is surrounded by PI cylinders. This observation is consistent with small angle X-ray scattering (SAXS) results of the specimen, shown in Figure 5.1b. While the scattering pattern of the as-made sample is reminiscent of a micellar arrangement of pores, the reflection peaks of the redissolved sample align well with a morphology of hexagonally arranged cylinders. This is a convincing indication that the approach resulted in a better solubility of the hybrid material, leading in combination with the slower evaporation of the solvents to a close resemblance of the phase separated hybrid material to a BCP morphology near thermodynamic equilibrium.
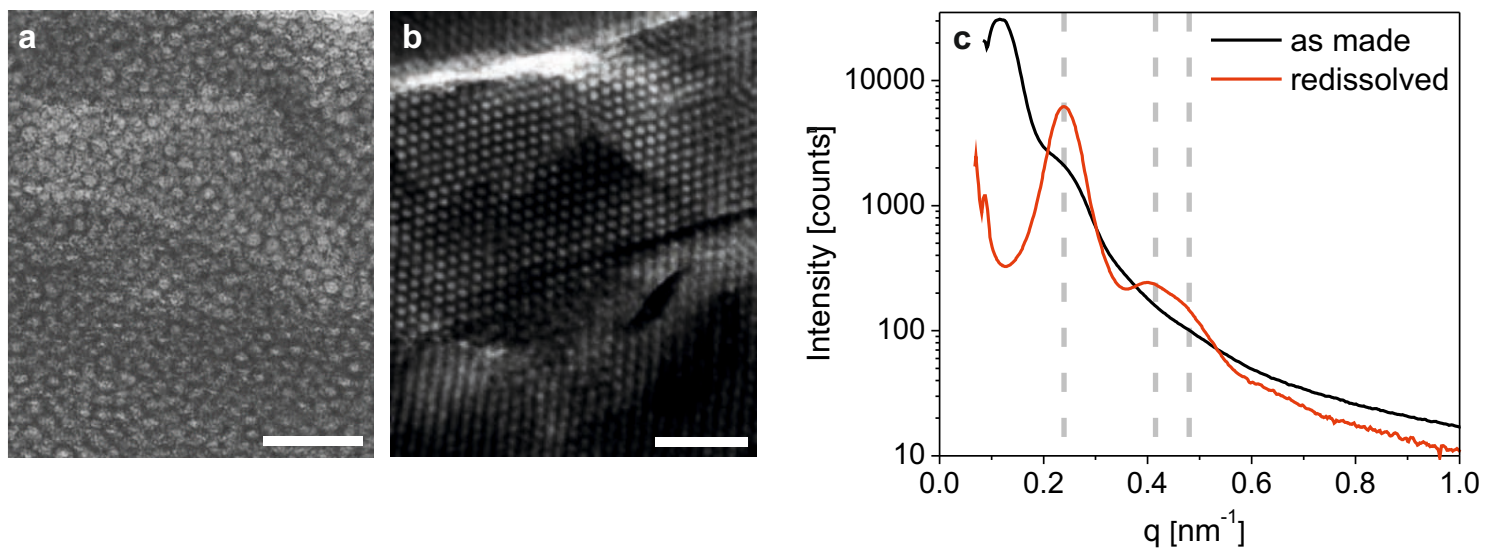

Figure 5.1: Bulk co-assembly of PI-b-PEO \& $\mathrm{TiO}_{2}$ : effect of annealing conditions. TEM images of samples that were made a) without and b) with the described redissolving step under otherwise identical experimental conditions. Scale bars represent $500 \mathrm{~nm}$. c) Radially integrated SAXS patterns of both samples, where $q=4 \pi \sin \theta / \lambda$ (see Chapter 4). The vertical lines correspond to a hexagonal arrangement of cylinders, which are observed for the redissolved sample. 
The versatility of this approach is demonstrated in Figure 5.2, where the mixing ratio of $\mathrm{TiO}_{2}$ and polymer in the initial solution was varied to access a range of morphologies. According to the TEM images, a 1:3 ratio, defined by the mass of the resulting $\mathrm{TiO}_{2}$ after calcination to the mass of polymer in solution, resulted in the formation of $\mathrm{TiO}_{2}$-rich cylinders surrounded by a PEO matrix. The vol\% of the PI block used in this study was $77 \%$, which means that the incorporated $\mathrm{TiO}_{2}$ must have swollen the PEO-rich phase by up to 8 vol\% to drive the hydrophilic block into a hexagonal arrangement of cylinders [22]. The observed morphology is consistent with SAXS scattering data, plotted in Figure 5.2d. The position of the reflection peaks predicted for a hexagonal morphology are indicated as grey lines. When increasing the inorganic weight fraction to 1:2, the morphology observed in TEM resembled a lamellar arrangement of the hydrophilic and hydrophobic components, shown in Figure 5.2b. Consistently, the SAXS reflection peaks align with the expected peak positions for the lamellar phase. Further increase of the $\mathrm{TiO}_{2}$ weight fraction to 3:2 resulted in an inverse hexagonal structure, where the $\mathrm{TiO}_{2}$ resides in the majority matrix, being surrounded by PI cylinders. Different projections of the cylinders can be seen in the image plane, which clearly distinguishes this morphology from an ordered micellar array. This is supported by the corresponding SAXS data shown in Figure 5.2f, where the radially integrated scattering pattern aligns well with the expected peak positions of a hexagonal morphology.

The results presented in Figures 5.1 and 5.2 illustrate the potential of introducing a redissolution procedure after partial evaporation of the primary solvent system. Yet, the success of this approach largely depends on the appropriate timing for evaporation and redissolution. If the condensation reaction has already completed during the first evaporation step, the material will no longer redissolve. On the other hand, interrupting the primary evaporation process too early may cause a deterioration of the structure formation process by residual water. In experimental practise, the appropriate timing proved relatively robust. The primary evaporation process was stopped when a sudden increase in viscosity inhibited further flow of residual solvent in the Petri dish. Subsequent work on understanding solubility parameters and the kinetics of the sol-gel reaction enabled Stefik et al. to introduce a one-pot approach based on the toluene/1-butanol azeotrope solvent system and hydrolysis under less acidic conditions [11]. Shifting from a conventional oversupply of $4 \mathrm{~mol} \mathrm{H}_{2} \mathrm{O}$ per mol of titanium alkoxide to a molar ratio of 1 in conjunction with the appropriate timing allowed to suppress the discussed micellisation issues and led to equilibrated morphologies without the need of a redissolving step. Yet, high $\mathrm{TiO}_{2}$ loadings, such as an inorganic to organic ratio of 3:1 led to the precipitation of the inorganic material. In contrast, the presented two step procedure enables highly inorganic mixtures up to a ratio of 5:1. This is an indication that during the primary evaporation cycle the $\mathrm{TiO}_{2}$ has already partially condensed on the local scale (typically 1-4 nm [23]), lim- 

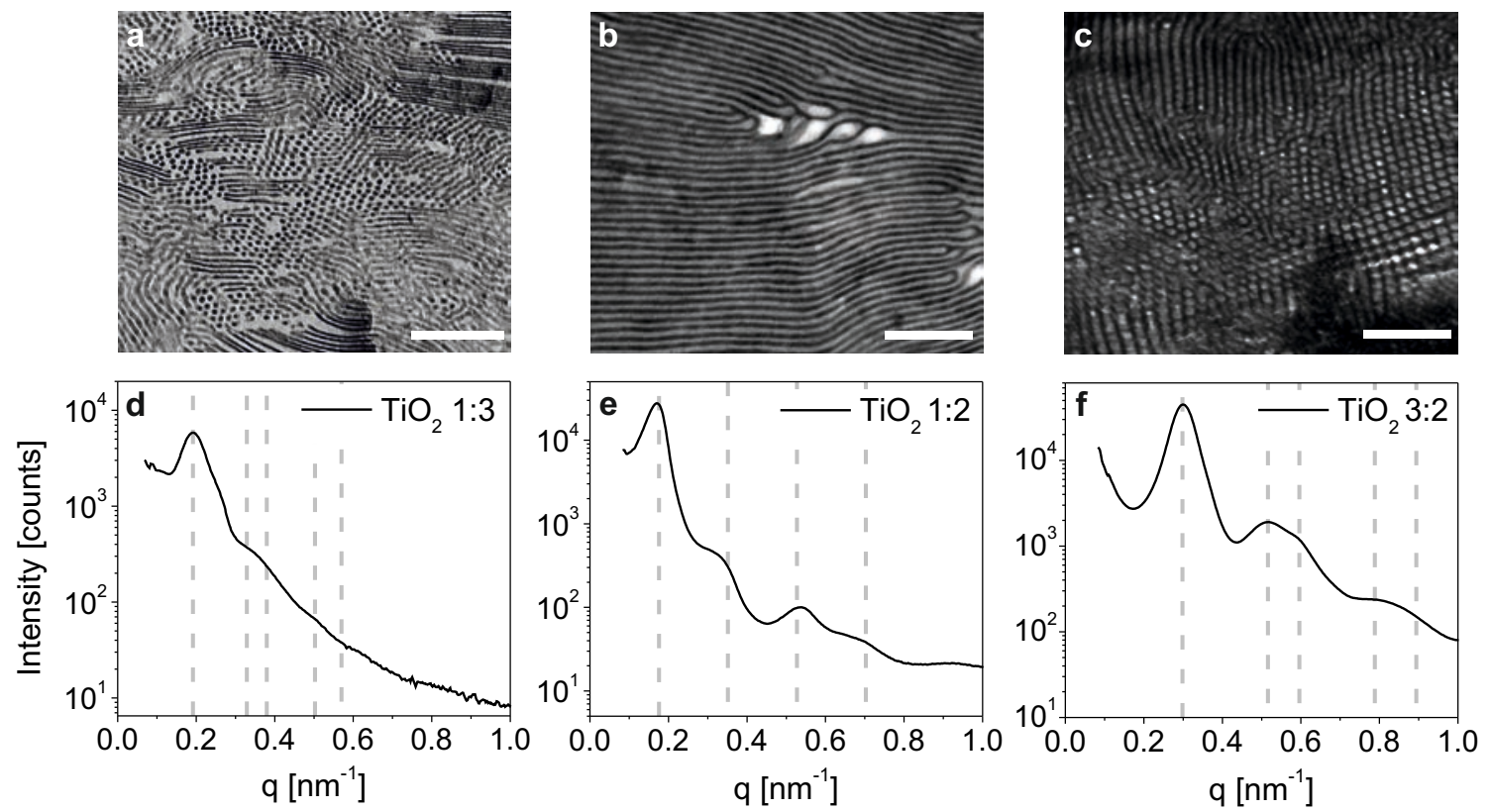

Figure 5.2: Bulk co-assembly of PI- $b$-PEO \& $\mathbf{T i O}_{2}$ : effect of mixing ratio. a) - c) Transmission electron micrographs of hybrid $\mathrm{PI}-b-\mathrm{PEO} / \mathrm{TiO}_{2}$ samples. Samples were derived from inorganic to organic mixing ratios of a) 1:3, b) 1:2 and c) 3:2, which led to a variation of the morphological arrangement from $\mathrm{TiO}_{2}$-rich cylinders in an organic matrix (1:3), to $\mathrm{TiO}_{2}$ lamellae (1:2) and for a high $\mathrm{TiO}_{2}$ loading (3:2), to organic cylinders in $\mathrm{TiO}_{2}$-rich inverse hexagonal matrix. Scale bars represent $500 \mathrm{~nm}$. d) - f) Corresponding radially integrated plots from SAXS of the samples. Expected peak positions for a d), f) hexagonal arrangement of cylinders, and e) lamellae are indicated as grey lines, calculated from the position of the first reflection peak.

iting the amount of water produced during the second evaporation. In addition, a redissolving step decouples material synthesis from film processing. It is therefore possible to redissolve the material in a well defined manner up to high concentrations $(<20 \mathrm{wt} \%)$, which greatly enhances control over thin film processing.

\subsubsection{Inorganic thin films with controlled pore size and porosity}

The morphology of a thin film that was processed via redissolution and spin-coated from an azeotrope solution is shown in Figure 5.3a. After deposition, the film was lifted onto a TEM grid for imaging. The observed morphology is consistent with a hexagonal arrangement of micellar pores. Corresponding characterisation of the specimen by SAXS is presented in Figure $5.3 \mathrm{~b}$ in form of a radially integrated plot of the scattering pattern. Bulk processing of the identical solution by solvent casting resulted in an inverse hexagonal $\mathrm{TiO}_{2}$ matrix, surrounding PI cylinders (Figure 5.1b). In contrast no long-range order can be observed in the hybrid film. The greatly increased evaporation kinetics during spin coating has presumably prevented the 
hybrid film from reaching thermodynamic equilibrium.

It is important to note that this micellar assembly represents a 3D bicontinuous network of $\mathrm{TiO}_{2}$ with pores on the 10-nm length scale, a morphology that offers isotropy and structural integrity. Both characteristics are not inherent to most BCP morphologies at thermodynamic equilibrium. An inverse hexagonal arrangement of cylinders is not isotropic and needs to be aligned for pore access. Other BCP morphologies, such as hexagonal inorganic cylinders, do not offer structural integrity as the features tend to cluster upon removal of the surrounding matrix thethicellar film morphologies proved reproducible and yielded promising results when used in dye-sensitised solar cells and optical coatings, this film formation strategy was further explored. Though micellar, the pores showed close-packing in a hexagonal arrangement and exhibited well defined optical interfaces. These characteristics are a direct consequence of the dissolution procedure. In contrast, when processing the films directly from the initial THFbased solution, the films were rough and of greatly varying thickness. This can be attributed to the volatility of THF, preventing the formation of a well-defined film during spin coating, see Section 4.1.3.

Control over pore dimensions, porosity and well-defined percolation paths are beneficial for many applications of mesoporous films. In the widely established nanoparticle-route, pores are generated by the random dense packing of nanometre-sized particles and subsequent sintering. The pore geometry is therefore defined by the remaining interstitial space between close-packed particles, which results in a highly heterogeneous size and shape distribution [25]. Pore generation in a SDA approach to mesoporous thin films is fundamentally different.
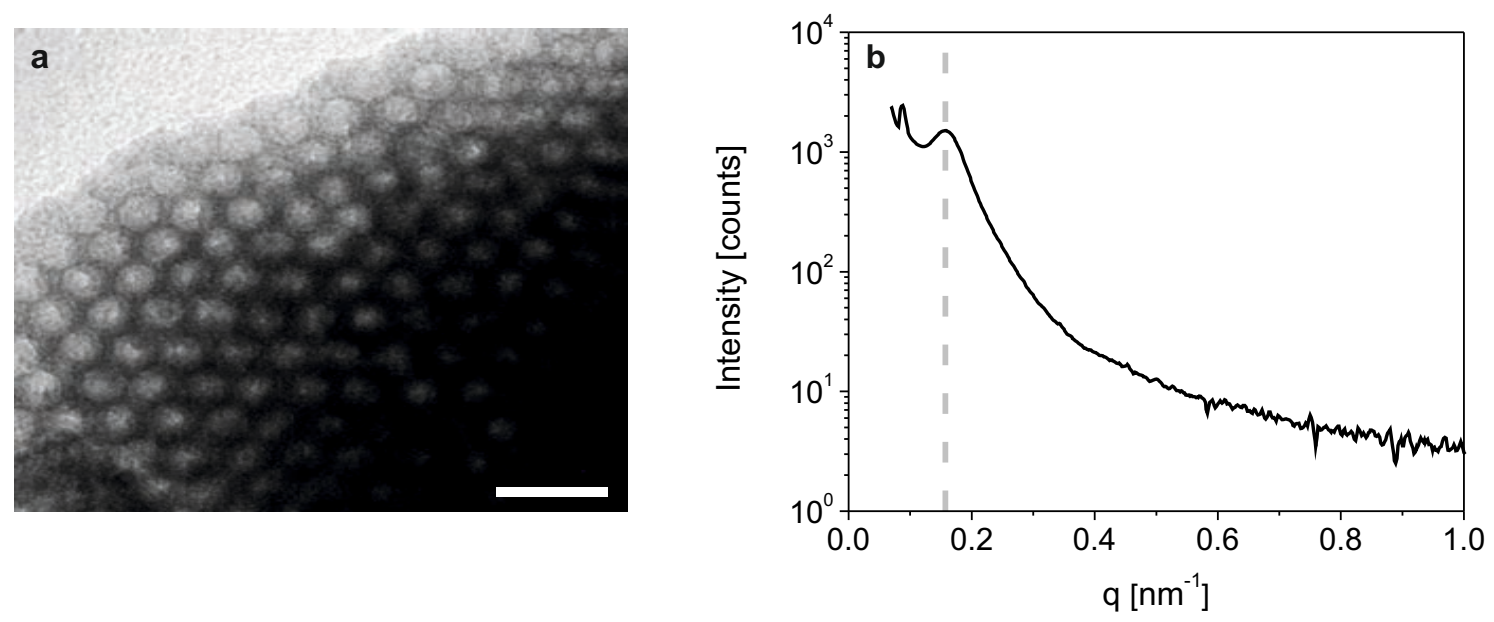

Figure 5.3: Thin film co-assembly of PI-b-PEO \& $\mathbf{T i O}_{2}$. a) TEM image of the ordered, hexagonally arranged micellar hybrid film produced by spin-coating the redissolved material. b) Corresponding radially integrated SAXS pattern of the specimen, where only the first order reflection peak was observed. 
The volume occupied by the sacrificial polymer block that incorporates no inorganic material will result in empty space upon calcination. Hence, the molecular weight of this block should be the main parameter to define the pore size distribution. In Figure 5.4a and d SEM top views of mesoporous silica-type films are presented, which were generated by SDAs of different molecular weights but identical mixing ratio of inorganic material and polymer (1:1 in weight). Both films exhibit a highly porous mesostructure, resembling an inverse opal morphology as known for larger length scales from colloidal templating (see Chapter 1.2.4). SEM images of porous inorganic films after the polymer has been removed by oxygen plasma etching or calcination are highly reminiscent of the hybrid morphology shown in Figure 5.4a. These findings are similar when using either $\mathrm{TiO}_{2}$ or aluinosilicate as the inorganic sol. The accessibility and continuity of the pores over several micellar planes is clearly discernible. No solid overlayer has formed that could impede the infiltration of the network.

Figure 5.4a-c illustrates the pore size analysis procedure for a mesoporous film stemming from a PI- $b$-PEO BCP of $\mathrm{M}_{\mathrm{n} \text {, tot }}=34.4 \mathrm{~kg} / \mathrm{mol}$ (71.6 vol\% PI). From the raw SEM top view micrograph a binary image was obtained by applying an intensity threshold. For comparison,
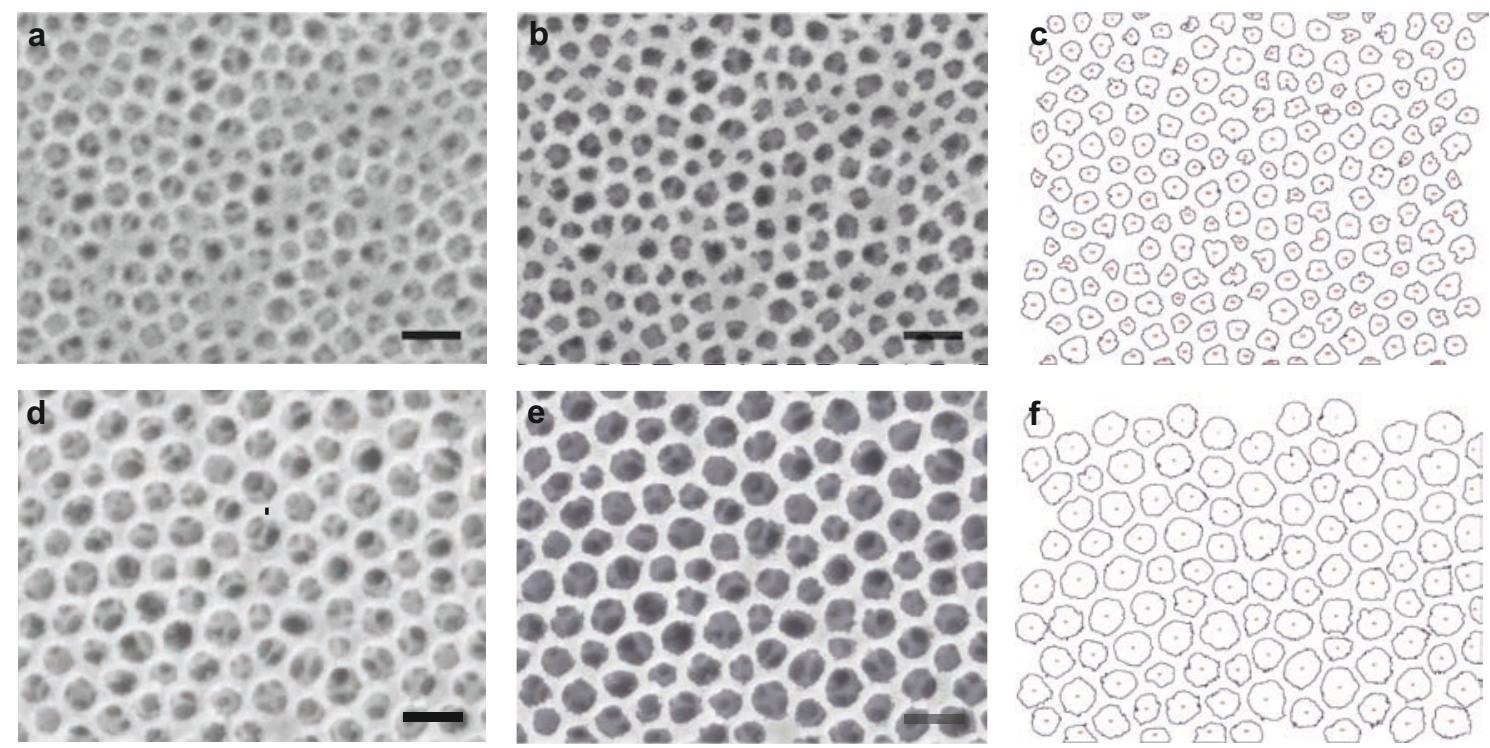

Figure 5.4: Pore size analysis in thin films. Pore size analysis for mesoporous silica-based films derived from the co-assembly of aluminosilicate sol and PI- $b$-PEO BCP. a) SEM top view micrograph of a mesoporous film that served for image analysis $\left(M_{n, \text { tot }}=34.4 \mathrm{~kg} / \mathrm{mol}\right)$. b) Superposition of original SEM image and an abstracted binary image, which was derived by applying an intensity threshold. c) Arithmetic pore identification by the "ImageJ" led to a pore size distribution of $33 \pm 6 \mathrm{~nm}$ [26]. For comparison, manual analysis yielded an average pore size of $34 \pm 4 \mathrm{~nm}$. d) - f) Image analysis for a mesoporous film derived from a BCP of $M_{\mathrm{n}}=91.6 \mathrm{~kg} / \mathrm{mol}$ led to pore size distribution of $52 \pm 6 \mathrm{~nm}$. This compares to a manual measurement of $52 \pm 4 \mathrm{~nm}$. The ratio of inorganic material to polymer was in both samples 1:1. Scale bars represent $100 \mathrm{~nm}$. 
the raw and binary image are superimposed in Figure 5.4b. The binary image was subsequently analysed with the image analysis software "ImageJ" [26], which yielded a pore size distribution of $33 \pm 6 \mathrm{~nm}$. Based on the raw image, the pore size distribution was also determined manually, rendering $34 \pm 4 \mathrm{~nm}$. In Figure 5.4d-e a similar pore size analysis is presented for an increased molecular weight of the pore forming PI block $\left(M_{n, \text { tot }}=91.6 \mathrm{~kg} / \mathrm{mol}, 68.8 \mathrm{vol} \%\right.$ PI). Here, a mean pore diameter of $53 \pm 6 \mathrm{~nm}$ resulted from image analysis and a distribution of $52 \pm 4 \mathrm{~nm}$ was measured manually. An increase of $\sim 55-60 \%$ is in good agreement with scaling laws of polymer chain statistics [27]. The radius of gyration for an unperturbed polymer chain scales with $\sqrt{N}$ (where $N$ is the degree of polymerisation), i.e. by a factor of 1.59 , when increasing the molecular weight of the PI block from $24.8 \mathrm{~kg} / \mathrm{mol}$ to $62.7 \mathrm{~kg} / \mathrm{mol}$.

In addition to the control over the resulting pore size distribution, control over the porosity is another important parameter for many applications of mesoporous thin films. Subject to structural integrity, the mixing ratio in the initial solution between inorganic sol and sacrificial SDA should determine the resulting porosity after calcination. The variation in porosity of an inorganic film can be characterised by optical means, as this parameter is reflected in the effective refractive index of the films. The experimentally found dependence of refractive index on the polymer weight fraction can be seen in Figure 5.5a. Samples series of different molecular weight polymers are plotted separately and show a similar dependence. Following the Bruggeman effective medium approximation (see Chapter 2.5), a quasi-linear relationship between the polymer weight fraction, the resulting pore volume and hence the corresponding refractive index is expected. In fact, the measured refractive index scales quasi-linearly for low polymer weight content. When increasing the organic fraction to $\geq 50 \mathrm{wt} \%$, the experimentally observed value deviates from the expected scaling. This is probably related to a progressive shrinkage of the film for an increasing polymer weight fraction. An organic weight content of $50 \%$ effectively corresponds to a polymer volume content of $\sim 67 \%$. It is therefore reasonable to assume that such high polymer volume fractions lead to a certain densification of the inorganic network after removal of the SDA. Nevertheless, the porosity could steadily be increased by extending the polymer weight fraction to $66 \%$. The measured refractive index was $n=1.135 \pm 0.007(\lambda=632 \mathrm{~nm})$, which corresponds to a relative pore volume of $\sim 71 \%$.

These results imply that the presented route allows to tune the porosity over a wide range, i.e. from $\sim 30 \%$ to over $70 \%$. This is in line with studies of Garcia et al. who reported that the hydrophilic PEO block can accommodate large amounts of guest material (up to 20 times its volume) via hydrogen bonds [4]. Similar porosity tuning was achieved for mesoporous $\mathrm{TiO}_{2}$, which will be presented in Chapter 8 . 

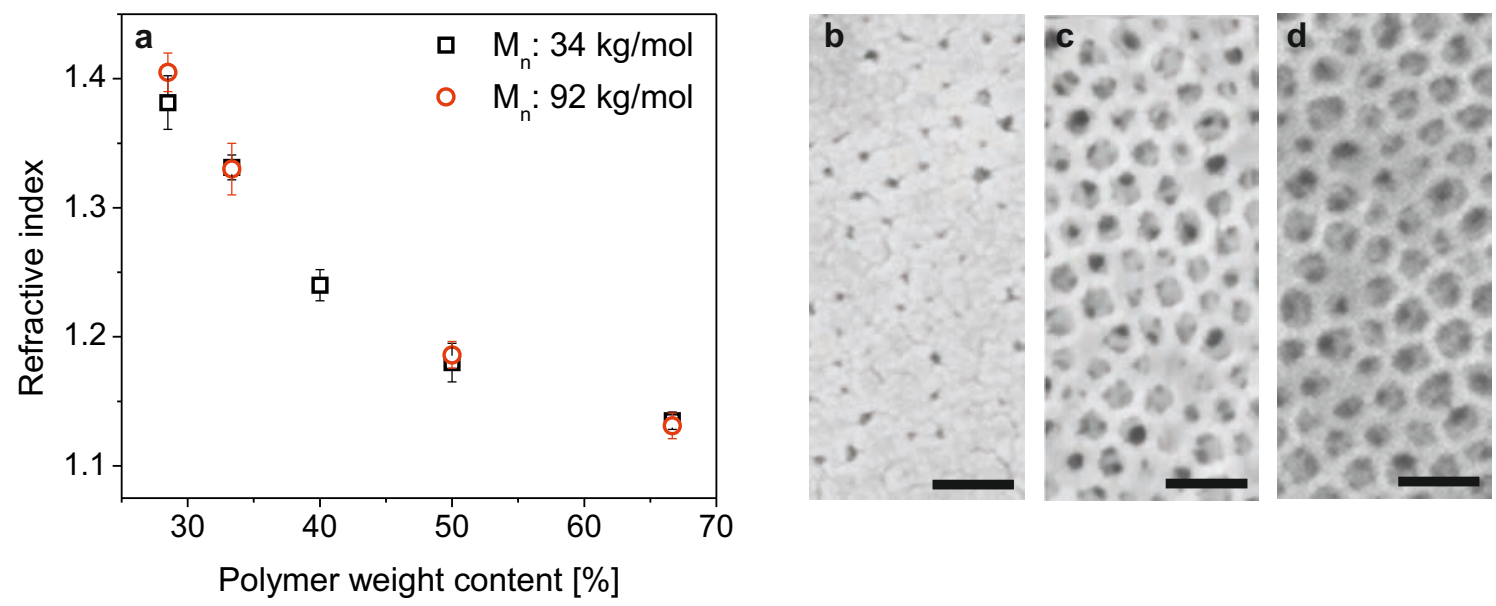

Figure 5.5: Porosity tuning of mesoporous films. a) Dependence of refractive index of the resulting inorganic film on the polymer weight content in the initial hybrid solution. Results for two polymers of different molecular weight are shown. The polymer weight fraction was determined as percentage with respect to the total weight of polymer SDA and resulting silicatype material. The refractive index values are shown for $\lambda=632 \mathrm{~nm}$. b) - d) Scanning electron microscopy top view of mesoporous silica-type films, stemming from a solution of PI- $b$-PEO BCP $\left(M_{\mathrm{n}}=34.4 \mathrm{~kg} / \mathrm{mol}\right)$ and aluminosilicate sol. The polymer weight fraction was b) $\left.33 \%, \mathbf{c}\right) 40 \%$ and d) $50 \%$. Scale bars represent $100 \mathrm{~nm}$.

\subsubsection{Highly ordered network morphologies in silica-based thin films}

The presented route to mesoporous films via dense packing of sacrificial micelles is robust in terms of processing conditions and offers fine control over porosity and pore size. However, some applications rely on a specific space symmetry of the materials configuration, which can only be achieved by BCP microphase separation close to thermodynamic equilibrium. One example are optical metamaterials, described in Chapter 2.5. Overcoming micellisation and kinetic trapping in thin films would enable to access the same range of BCP equilibrium morphologies as reported in this chapter for bulk materials. Materials arrangement of particular interest are continuous multiple network structures, such as the double gyroid. These phases do not have the discussed drawbacks of other BCP equilibrium morphologies, such as domain alignment, pore accessibility and structural collapse [28]. Extending from an AB diblock copolymer to an $\mathrm{ABC}$ terblock copolymer greatly increases the gyroid composition window in the phase diagram, from $2-6 \%$ in an $\mathrm{AB}$ to $4-14 \%$ in ABC systems $[29,30]$. Furthermore, a ternary system offers several configurations of the gyroid morphology. One example is the double gyroid, with an ABCBA core-shell structure (space group: $l a \overline{3} d, \mathrm{Q}^{230}$ ), another one the chiral alternating gyroid, where two individual networks $\mathrm{A}$ and $\mathrm{C}$ are embedded in a matrix of B (space group: $l l 4_{1} 32, \mathrm{Q}^{214}$ ).

An ABC terblock copolymer in form of a PI- $b$-PS- $b$-PEO chain structure has already been 
used as a SDA for transition metal oxides, in particular $\mathrm{TiO}_{2}$ and $\mathrm{Nb}_{2} \mathrm{O}_{5}$ [31]. After removal of the polymer, porous inorganic morphologies were observed in the bulk material which closely resembled the corresponding equilibrium morphologies of the BCP melt, i.e. chiral network structures, such as the non-centrosymmetric alternating gyroid $\mathrm{Q}^{214}$ as well as centrosymmetric network morphologies.

The aim of the present study was the extension of these results to thin films. For this purpose, the previously described evaporation-controlled blade coating technique was developed, see Section 4.1.3. Basically, a commercially available set-up was modified by the integration of a programmable heating element and an enclosed chamber with solvent-rich atmosphere, fed by a saturated carrier gas. As a result, the evaporation kinetics of the solvent were greatly reduced due to the increased partial pressure in the saturated environment. Controlling the substrate temperature during deposition allowed to further promote phase separation by increasing the chain mobilities. Based on results from solvent-casting of thick films, a temperature of $50{ }^{\circ} \mathrm{C}$ was found to optimise the competing kinetics of polymer phase separation and gelation of the inorganic material [11,31].

In the experiments the same PI- $b$-PS- $b$-PEO terblock copolymer was used as in reference [31]. The mixing ratio between sol and polymer was adjusted to result in the phase space of the alternating gyroid structure in the ternary diagram [30]. The aluminosilicate should therefore reside in the hydrophilic minority component $\mathrm{C}$, leading to an inorganic single gyroid network upon removal of the SDA. Figure 5.6 shows an overview of cross-sectional SEM images for different silica-type thin film morphologies after exposure to an oxygen plasma. When blade coated in an unsaturated solvent atmosphere from a toluene/1-butanol azeotrope solution, the films exhibited a random network structure that showed the expected porosity but did not resemble the expected equilibrium morphology (Figure 5.6a). The use of an enclosed chamber with saturated solvent atmosphere during the coating process significantly slowed down the drying process from less than a minute to over $15 \mathrm{~min}$ for a $\mu \mathrm{m}$-thick film. The increased drying time resulted in a more equilibrated structure with indications of the expected network morphology, as discernible in Figure 5.6c.

A change of the solvent from the toluene/1-butanol azeotrope system to anisole further improved structure control and resulted in a morphology that closely resembled an alternating gyroid formed by the silica-type material (Figure 5.6d). Anisole has a boiling point of $154{ }^{\circ} \mathrm{C}$, further extending the drying time during which microphase separation can take place. As a result, films consistently exhibited high structural order throughout the film. Yet, at the bottom interface a lamellar arrangement was observed, which can be attributed to a preferential interaction of the polar PEO/inorganic phase with the substrate [32,33]. Dipcoating in a solution of octadecyltrichlorosilane in cyclohexane greatly reduced the surface polarity due to 
the formation of a silane monolayer on the substrate. Films prepared on silanised substrates showed no discontinuity of the network structure at the substrate interface, as evidenced in Figure 5.6f. The long-range order of the obtained network structures is depicted in Figure $5.6 \mathrm{~g}$.

Further characterisation, e.g. by grazing incidence SAXS, is required to confirm possible non-centrosymmetric configurations of the structural unit cell. In principle, such network structures should be ideally suited for applications in optical films, where linear dichroism and gyrotropic light propagation have been observed for chiral alternating gyroid templates upon infiltration of the network with gold and subsequent removal of the polymer [34]. Shifting
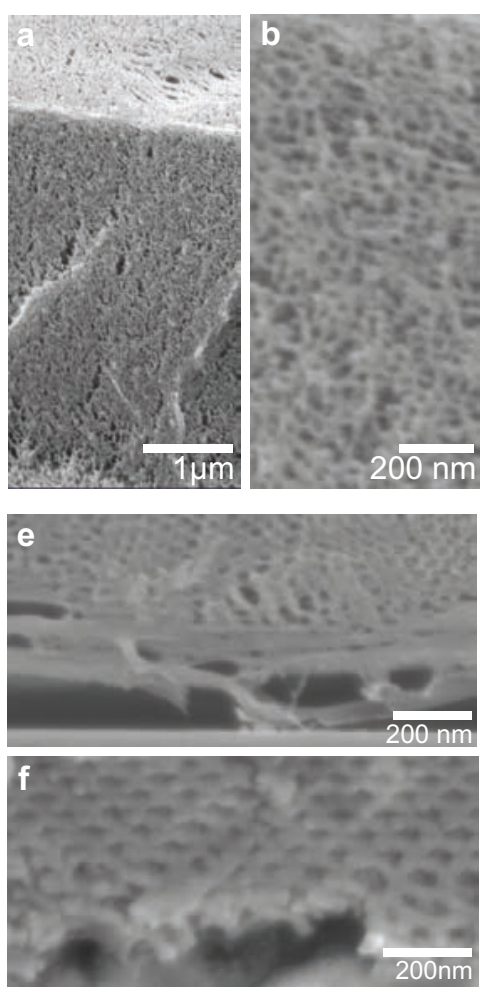
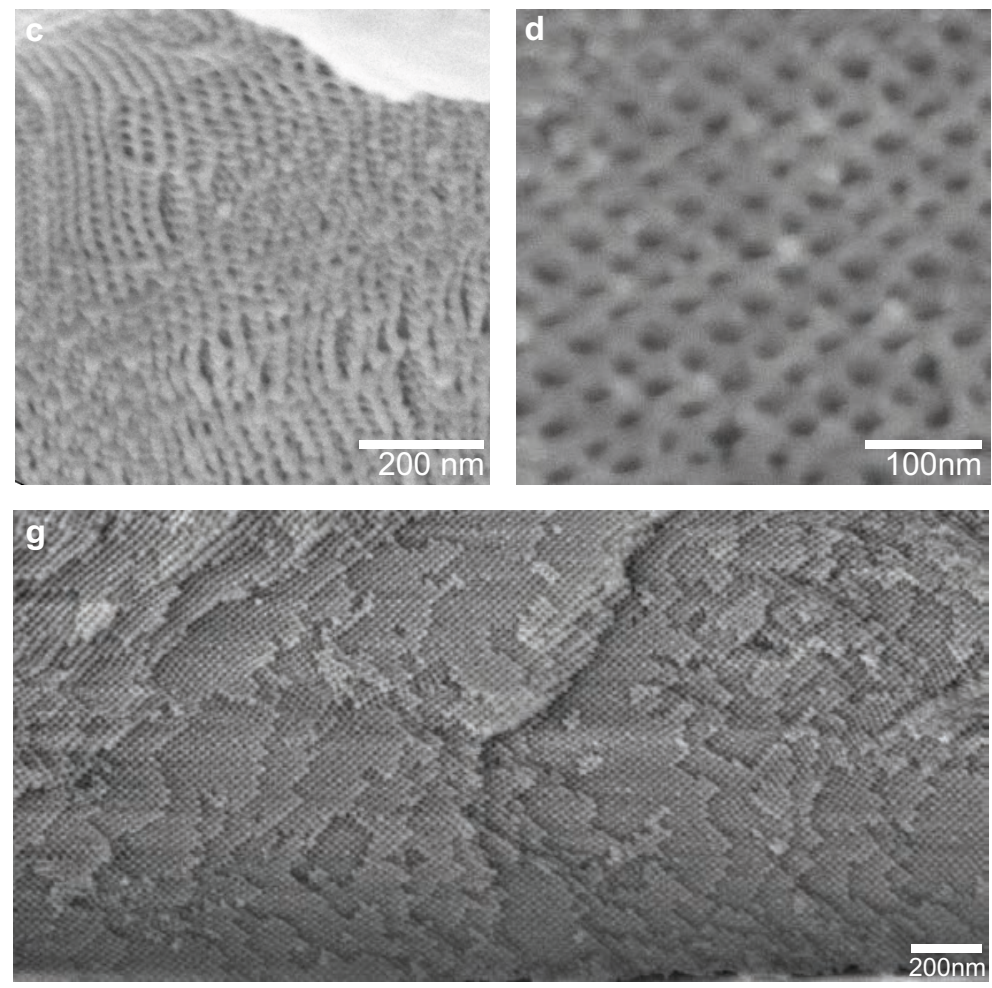

Figure 5.6: Assembly of silica-type thin films into highly ordered network morphologies. The $\mathrm{PEO} /$ aluminosilicate content was adjusted to a volume fraction of $30 \%$ which should result in an alternating gyroid morphology under equilibrium conditions [31]. After deposition and annealing, films were exposed to an oxygen plasma to remove the SDA. All cross-sectional imaging was carried out by SEM. a) - b) Mesoporous film prepared from a toluene/1-butanol azeotrope solution in unsaturated atmosphere. c) Film prepared in saturated solvent atmosphere under otherwise identical conditions. d) - g) Resulting morphologies obtained by the use of anisole and deposition in a saturated atmosphere. d) Close-up view of the resulting network morphology. e) Preferential interaction of the polar PEO/inorganic phase with the substrate led to the formation of lamellae at the substrate interface. f) After silanisation of the substrate, the bottom interface shows no indication of preferential interaction. g) Zoomed-out view of highly ordered network morphology in thin a film. 
from the previously reported organic to an inorganic template may greatly improve the longterm stability, in terms of temperature and abrasion resistance as well preventing oxidation of the plasmonic metals, such as silver.

Silica-type networks offer high temperature stability. Crystallisation accompanied by structural collapse only occurs well above $1000^{\circ} \mathrm{C}$. The presented mesoporous films are therefore ideal candidates for the crystallisation of infiltrated functional materials at high temperatures $[35,36]$. The "hard" scaffold may subsequently be removed by wet etching with hydrogen fluoride to reveal a free-standing film of the infiltrated material after crystallisation at temperatures that would otherwise lead to structural collapse [37].

Reduction of the aluminosilicate film in magnesium vapour may lead to the formation of silicon domains in a free-standing network [38]. This would make the materials system ideally suited as anodes in lithium-ion batteries [39]. Insertion of lithium ions typically poses a major challenge for the anode due to the imposed volume change for every charging and discharging cycle [40]. The length scale of the SDA-directed structure, the dispersion of aluminium-rich and silicon-rich domains and the structural integrity of a gyroid network upon volume contraction make this route ideally suited to improve anode stability and capacity.

\subsection{Conclusions}

Several strategies are presented in this chapter that are geared towards controlling structure formation of inorganic materials by BCP co-assembly with PI- $b$-PEO. The use of solvent exchange enabled to overcome kinetic barriers for structure formation of sol-gel-derived $\mathrm{TiO}_{2}$. The resulting hybrid material arrangement of hydrophobic $\mathrm{PI}$ and hydrophilic $\mathrm{PEO} / \mathrm{TiO}_{2}$ beared close resemblance with typical BCP morphologies in the melt at thermodynamic equilibrium. By the variation of the mixing ratio between the inorganic guest and the polymer host in solution, different morphologies were obtained, such as hexagonal $\mathrm{TiO}_{2}$-rich cylinders in an organic matrix, $\mathrm{TiO}_{2}$ lamellae or a $\mathrm{TiO}_{2}$ inverse hexagonal matrix.

In thin films, processing via redissolution of the hybrid material led to the formation of ordered micelles in a close-packed lattice. Tuning of the BCP's molecular weight and mixing ratio between inorganic sol and organic SDA allowed fine tuning of the porosity and refractive index of the resulting mesoporous films after removal of the polymer host. While the micellar assembly does not form at thermodynamic equilibrium, it represents a very promising route to mesoporous films. A hexagonal arrangement of pores offers morphological isotropy and structural integrity and allows full accessibility of the porous network. Processing is greatly simplified and highly robust with respect to the various parameters. In addition, the proposed redissolving step decouples material synthesis from film processing and therefore allows pre- 
cise control of the resulting film thickness.

Thin film deposition by evaporation-controlled blade coating prevented micellisation and enabled the realisation of highly ordered and free standing network structures in silica-type films. The high porosity, accessibility and fine structural order of the network makes the films an excellent candidate for a variety of applications, such as optical films, high temperature scaffolds and lithium-ion batteries.

\section{Bibliography}

[1] S. Bagshaw, E. Prouzet, and T. Pinnavaia, "Templating of mesoporous molecular-sieves by nonionic polyethylene oxide surfactants," Science, vol. 269, no. 5228, pp. 1242-1244, 1995.

[2] S. Boettcher, J. Fan, C.-K. Tsung, Q. Shi, and G. Stucky, "Harnessing the sol-gel process for the assembly of non-silicate mesostructured oxide materials," Accounts Of Chemical Research, vol. 40, no. 9, pp. 784-792, 2007.

[3] M. Templin, A. Franck, A. DuChesne, H. Leist, Y. Zhang, R. Ulrich, V. Schädler, and U. Wiesner, "Organically modified aluminosilicate mesostructures from block copolymer phases," Science, vol. 278, no. 5344, pp. 1795-1798, 1997.

[4] B. Garcia, M. Kamperman, R. Ulrich, A. Jain, S. Gruner, and U. Wiesner, "Morphology diagram of a diblock copolymer-aluminosilicate nanoparticle system," Chemistry $O f$ Materials, vol. 21, no. 22, pp. 5397-5405, 2009.

[5] P. Alberius, K. Frindell, R. Hayward, E. Kramer, G. Stucky, and B. Chmelka, "General predictive syntheses of cubic, hexagonal, and lamellar silica and titania mesostructured thin films," Chemistry Of Materials, vol. 14, no. 8, pp. 3284-3294, 2002.

[6] X. Liu, B. Tian, C. Yu, F. Gao, S. Xie, B. Tu, R. Che, L. Peng, and D. Zhao, "Roomtemperature synthesis in acidic media of large-pore three-dimensional bicontinuous mesoporous silica with Ia3d symmetry," Angewandte Chemie - Int. Ed., vol. 41, no. 20, pp. 3876-3878, 2002.

[7] P. Mokarian-Tabari, M. Geoghegan, J. R. Howse, S. Y. Heriot, R. Thompson, and R. Jones, "Quantitative evaluation of evaporation rate during spin-coating of polymer blend films: control of film structure through defined-atmosphere solvent-casting," $E u$ ropean Physical Journal E, vol. 33, no. 4, pp. 283-289, 2010.

[8] C. Brinker and G. Scherer, Sol-Gel Science: The Physics and Chemistry of Sol-Gel Processing. Academic Press, 1st ed., 1990.

[9] V. Urade, T.-C. Wei, M. Tate, J. Kowalski, and H. Hillhouse, "Nanofabrication of doublegyroid thin films," Chemistry Of Materials, vol. 19, no. 4, pp. 768-777, 2007.

[10] P. Du, M. Li, K. Douki, X. Li, C. Garcia, A. Jain, D. Smilgies, L. Fetters, S. Gruner, U. Wiesner, and C. Ober, "Additive-driven phase-selective chemistry in block copolymer thin films: the convergence of top-down and bottom-up approaches," Advanced Materials, vol. 16, no. 12, pp. 953-957, 2004. 
[11] M. Stefik, J. Song, H. Sai, M. Orilall, S. Guldin, P. Boldrighini, U. Steiner, S. Gruner, and U. Wiesner, "Ordered mesoporous titania from highly amphiphilic block copolymers: tuned solution conditions enable morphology control." manuscript in preparation.

[12] G. Wanka, H. Hoffmann, and W. Ulbricht, "Phase-diagrams and aggregation behavior of poly(oxyethylene)-poly(oxypropylene)-poly(oxyethylene) triblock copolymers in aqueous-solutions," Macromolecules, vol. 27, no. 15, pp. 4145-4159, 1994.

[13] D. Zhao, J. Feng, Q. Huo, N. Melosh, G. Fredrickson, B. Chmelka, and G. Stucky, "Triblock copolymer syntheses of mesoporous silica with periodic 50 to 300 angstrom pores," Science, vol. 279, no. 5350, pp. 548-552, 1998.

[14] I. Hamley, V. Castelletto, Z. Yang, C. Price, and C. Booth, "Melt phase behavior of poly(oxyethylene)-poly(oxypropylene) diblock copolymers," Macromolecules, vol. 34, no. 12 , pp. 4079-4081, 2001.

[15] A. Thomas, H. Schlaad, B. Smarsly, and M. Antonietti, "Replication of lyotropic block copolymer mesophases into porous silica by nanocasting: learning about finer details of polymer self-assembly," Langmuir, vol. 19, no. 10, pp. 4455-4459, 2003.

[16] G. Floudas, R. Ulrich, and U. Wiesner, "Microphase separation in poly(isoprene-bethylene oxide) diblock copolymer melts. I. Phase state and kinetics of the order-to-order transitions," Journal Of Chemical Physics, vol. 110, no. 1, pp. 652-663, 1999.

[17] P. Simon, R. Ulrich, H. Spiess, and U. Wiesner, "Block copolymer-ceramic hybrid materials from organically modified ceramic precursors," Chemistry Of Materials, vol. 13, no. 10, pp. 3464-3486, 2001.

[18] J. Lee, M. Orilall, S. Warren, M. Kamperman, F. DiSalvo, and U. Wiesner, "Direct access to thermally stable and highly crystalline mesoporous transition-metal oxides with uniform pores.," Nature Materials, vol. 7, no. 3, pp. 222-8, 2008.

[19] D. Morselli, M. Messori, and F. Bondioli, "Poly(methyl methacrylate)- $\mathrm{TiO}_{2}$ nanocomposite obtained by non-hydrolytic sol-gel synthesis," Journal Of Materials Science, vol. 46, no. 20, pp. 6609-6617, 2011.

[20] K. Pinder, "Activity of water in solution with tetrahydrofuran," Journal Of Chemical And Engineering Data, vol. 18, no. 3, pp. 275-277, 1973.

[21] J. Ponton, “Azeotrope databank.” http://eweb.chemeng.ed.ac.uk/, 2001.

[22] C. Park, J. Yoon, and E. Thomas, "Enabling nanotechnology with self assembled block copolymer patterns," Polymer, vol. 44, no. 22, pp. 6725-6760, 2003.

[23] S. Guldin, "Nanostructuring inorganic material by copolymer-assisted self-assembly and its multifunctional use for dye-sensitised solar cells," Master's thesis, Technische Universität München, 2008.

[24] E. Crossland, M. Nedelcu, C. Ducati, S. Ludwigs, M. Hillmyer, U. Steiner, and H. Snaith, "Block copolymer morphologies in dye-sensitized solar cells: probing the photovoltaic structure-function relation," Nano Letters, vol. 9, no. 8, pp. 2813-2819, 2009.

[25] S. Burnside, V. Shklover, C. Barbé, P. Comte, F. Arendse, K. Brooks, and M. Grätzel, "Self-organization of $\mathrm{TiO}_{2}$ nanoparticles in thin films," Chemistry Of Materials, vol. 10, no. 9, pp. 2419-2425, 1998. 
[26] W. Rasband, “ImageJ version 1.44o.” http://rsbweb.nih.gov/ij/, 2011.

[27] M. Rubinstein and R. Colby, Polymer physics. Oxford University Press, 1st ed., 2003.

[28] E. Crossland, M. Kamperman, M. Nedelcu, C. Ducati, U. Wiesner, D.-M. Smilgies, G. Toombes, M. Hillmyer, S. Ludwigs, U. Steiner, and H. Snaith, "A bicontinuous double gyroid hybrid solar cell,” Nano Letters, vol. 9, no. 8, pp. 2807-2812, 2009.

[29] F. Bates, M. Schulz, A. Khandpur, S. Förster, J. Rosedale, K. Almdal, and K. Mortensen, "Fluctuations, conformational asymmetry and block-copolymer phase-behavior," Faraday Discussions, vol. 98, pp. 7-18, 1994.

[30] T. Epps, E. Cochran, T. Bailey, R. Waletzko, C. Hardy, and F. Bates, "Ordered network phases in linear poly (isoprene-b-styrene-b-ethylene oxide) triblock copolymers," Macromolecules, vol. 37, pp. 8325-8341, 2004.

[31] M. Stefik, S. Wang, R. Hovden, H. Sai, M. Tate, D. Muller, U. Steiner, S. Gruner, and U. Wiesner, "Networked and chiral nanocomposites from ABC triblock terpolymer coassembly with transition metal oxide nanoparticles," Journal Of Materials Chemistry, vol. 22, no. 3, pp. 1078-1087, 2012.

[32] T. Russell, G. Coulon, V. Deline, and D. Miller, "Characteristics of the surface-induced orientation for symmetric diblock PS/PMMA copolymers," Macromolecules, vol. 22, no. 12 , pp. 4600-4606, 1989.

[33] J. Heier, E. Kramer, S. Walheim, and G. Krausch, "Thin diblock copolymer films on chemically heterogeneous surfaces," Macromolecules, vol. 30, no. 21, pp. 6610-6614, 1997.

[34] S. Vignolini, N. A. Yufa, P. S. Cunha, S. Guldin, I. Rushkin, M. Stefik, K. Hur, U. Wiesner, J. J. Baumberg, and U. Steiner, "A 3D optical metamaterial made by self-assembly," Advanced Materials, vol. 24, no. 10, 2012.

[35] J. Kondo and K. Domen, "Crystallization of mesoporous metal oxides," Chemistry Of Materials, vol. 20, no. 3, pp. 835-847, 2008.

[36] J. Brillet, M. Grätzel, and K. Sivula, "Decoupling feature size and functionality in solution-processed, porous hematite electrodes for solar water splitting," Nano Letters, vol. 10, no. 10, pp. 4155-4160, 2010.

[37] H. Arora, P. Du, K. Tan, J. Hyun, J. Grazul, H. Xin, D. Muller, M. Thompson, and U. Wiesner, "Block copolymer self-assembly-directed single-crystal homo- and heteroepitaxial nanostructures," Science, vol. 330, no. 6001, pp. 214-219, 2010.

[38] B. Hatton, L. Mishchenko, S. Davis, K. Sandhage, and J. Aizenberg, "Assembly of large-area, highly ordered, crack-free inverse opal films," Proceedings Of The National Academy Of Sciences Of The United States Of America, vol. 107, no. 23, pp. 1035410359, 2010.

[39] E. Richman, C. Kang, T. Brezesinski, and S. Tolbert, "Ordered mesoporous silicon through magnesium reduction of polymer templated silica thin films," Nano Letters, vol. 8, no. 9, pp. 3075-3079, 2008.

[40] B. Key, R. Bhattacharyya, M. Morcrette, V. Seznec, J.-M. Tarascon, and C. Grey, "Realtime NMR investigations of structural changes in silicon electrodes for lithium-ion batteries," Journal of The American Chemical Society, vol. 131, no. 26, pp. 9239-9249, 2009. 



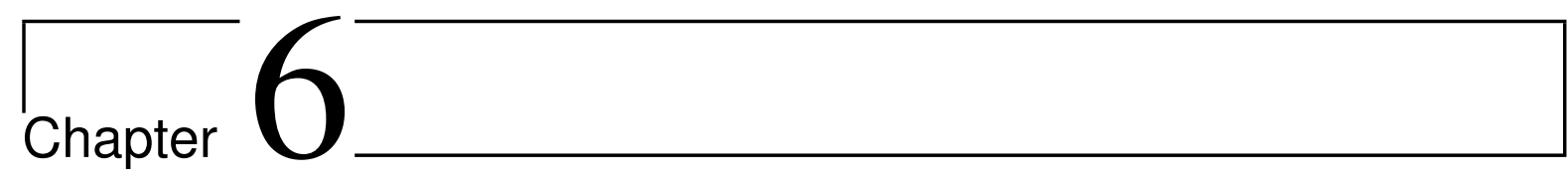

\title{
Crystal growth in block \\ copolymer-derived mesoporous $\mathrm{TiO}_{2}$
}

\begin{abstract}
Published by Stefan Guldin et al. in Energy \& Environmental Science (volume 4, page 225-233, 2011) [1]. See also "Charge transport limitations in self-assembled $\mathrm{TiO}_{2}$ photoanodes for solid-state dye-sensitized solar cell", manuscript under review by Dr. Pablo Docampo [2].
\end{abstract}

\subsection{Introduction}

The interest in nanocrystalline, mesoporous $\mathrm{TiO}_{2}$ has intensified in recent years for a variety of "green" applications such as dye-sensitised solar cells (DSCs) [3], photocatalysis [4], electrochemical capacitors [5], sensors [6] and electrochromic devices [7]. Typically, a mesoporous film is obtained by the sintering of $10-20 \mathrm{~nm}$ sized $\mathrm{TiO}_{2}$ nanoparticles that are predominatly of the anatase crystal structure [8]. The resulting network of randomly packed nanocrystals and intermediate pores expands the surface area of the film by a multitude (up to 1000 fold [3]), but this comes at the expense of greatly deteriorated electronic properties. While the electron mobility in single crystalline anatase $\mathrm{TiO}_{2}$ is usually on the order of $\mu \sim 10 \mathrm{~cm}^{2} / \mathrm{Vs}[9,10]$, this value decreases by orders of magnitude to around $\mu \sim 10^{-1}-10^{-5} \mathrm{~cm}^{2} / \mathrm{Vs}$ in nanoparticle films $[11,12,13]$. The reasons for the diminished electron transport in nanoparticle films are numerous. The high density of traps, predominantly located at grain boundaries and at the particles' surfaces, cause generated electrons to mostly populate localised states below the conduction band and only diffuse towards the electrode in iterative cycles of thermal detrapping and trapping $[14,15]$ (see Chapter 3.1 for further details). While low electron mobility was previously not a major limiting factor, new technologies, such as solid state dye-sensitised 
solar cells (ss-DSCs), are suffering from poor charge carrier transport, which appears to limit charge collection and thereby power conversion efficiency [16].

Novel electrode architectures have evolved in recent years with the aim to improve the electronic properties of the $\mathrm{TiO}_{2}$ photoelectrode (see Chapter 3.1). Furthermore, the poor morphological control in nanoparticle networks [8] may impede mass transport of bulky molecules, such as reactants and certain redox couples, which limits the performance of photocatalytic devices and DSCs $[17,18]$. In other device types, such as ss-DSCs, the smallest pores represent a barrier for effective infiltration of the porous film by other functional materials [19].

The use of block copolymers (BCPs) to guide the morphological arrangement of mesoporous $\mathrm{TiO}_{2}$ is a promising concept for improved structure control [20, 21, 22]. This has also led to remarkable results in DSC systems, at least for film thicknesses below $1 \mu \mathrm{m}[23,24]$. Yet, BCP-templated or co-assembled morphologies typically suffer from poor crystallinity and electronic properties that are correlated to the limited structural stability at high crystallisation temperatures. In order to prevent the collapse of the morphology, calcination is usually carried out at temperatures around $400^{\circ} \mathrm{C}$, which results in poor overall crystallinity in comparison to sintered nanoparticle films $[25,26]$. Transformation into a fully crystalline material at high calcination temperatures often leads to the collapse of the structure [26]. This can be attributed to the fact that nanocrystals grow at elevated crystallisation temperatures to diameters of $10-20 \mathrm{~nm}$. This exceeds by far the length scale of the widely used PEO- $b$-PPO$b$-PEO (Pluronic) structure-directing agent (SDA) and therefore causes the micro-morphology to break down during crystallisation. Alternative concepts include backfilling the mesopores with carbon or silica [27] or elaborate annealing and crystallisation protocols [21] to enhance temperature stability. The addition of preformed nanocrystals in order to decrease the crystallisation temperature of amorphous $\mathrm{TiO}_{2}$ is another promising concept, but it remains a challenge to simultaneously achieve high crystallinity and structural integrity [28]. Furthermore phase impurities have been reported in this materials system with finite amounts of $\mathrm{TiO}_{2}(\mathrm{~B})$ and traces of rutile, both of which are not favourable for solar cell operation $[29,30]$.

Recently, BCP systems of higher molecular weight were introduced, such as PHB- $b$-PEO. The increased pore walls, compared to Pluronic-derived materials, allow crystallisation at up to $700{ }^{\circ} \mathrm{C}$ for periods of $10 \mathrm{~min}[22,26]$. The introduction of this new SDA therefore enabled the increase of anatase phase fraction within the mesoporous morphology from previously reported $40 \%$ to $100 \%$. On the other hand, pore size $(\sim 8 \mathrm{~nm})$ and porosity $(\sim 30 \%)$ are still limited. This is a consequence of the relatively low molecular weight and the low volume fraction of the pore forming PHB block $\left(M_{\mathrm{n}, \text { tot }} \sim 8-10 \mathrm{~kg} / \mathrm{mol}\right.$; vol\% $\left.\%_{\text {PHB }} \sim 43-63 \%\right)$. Recent studies on the use of PI- $b$-PEO as SDA for the co-assembly of inorganic materials have pointed out several intrinsic advantages of this particular BCP system [31, 32, 33]. The 
well-established polymerisation procedure enables synthesis of large molecular weight BCPs (up to $M_{\mathrm{n} \text {, tot }} \sim 100 \mathrm{~kg} / \mathrm{mol}$ ) with a high fraction of the pore forming PI-block (typically around $75-90 \%)$ and a relatively narrow size distribution $(D \sim 1.02-1.05)$ [34, 35]. In principle, this should allow the development of mesoporous architectures that enable extended crystallite growth and exhibit pores large enough for infiltration and mass transport through the inorganic network.

In this chapter, the evolution of crystal growth in highly ordered mesoporous $\mathrm{TiO}_{2}$ films is investigated. The kinetics of the crystallisation process was resolved in situ by wide angle $\mathrm{X}$ ray scattering (WAXS), from the sol-gel-derived amorphous $\mathrm{TiO}_{2}$ to highly crystalline anatase. Furthermore, impedance spectroscopy and the implementation of porous $\mathrm{TiO}_{2}$ films in liquid electrolyte and ss-DSCs were employed to correlate the evolution of crystallinity with electron transport properties and photovoltaic performance of devices.

\subsection{Experimental}

\section{Material fabrication}

A titanium containing sol was prepared by the rapid addition of $0.69 \mathrm{ml} \mathrm{HCl} \mathrm{(37 \% )} \mathrm{to} 1 \mathrm{ml}$ titanium ethoxide (purum) under vigorous stirring. A solution of $0.1 \mathrm{~g}$ poly(isoprene-blockethylene oxide) copolymer (PI- $b$-PEO) $\left(\mathrm{M}_{\mathrm{n}}=35.7 \mathrm{~g} / \mathrm{mol}, 23 \mathrm{wt} \%\right.$ PEO$)$ in $7 \mathrm{ml}$ of tetrahydrofuran (THF) was prepared alongside. After $90 \mathrm{~min}$ of stirring, $0.87 \mathrm{ml}$ of the resulting sol was added to the polymer solution, before the volatile components were evaporated at $50^{\circ} \mathrm{C}$ in a Petri dish. Shortly after, the dry material was redissolved in an azeotrope solvent mixture of $72.8 \mathrm{wt} \%$ toluene $(99.8 \%$ ) and $27.2 \mathrm{wt} \%$ 1-butanol (99\%). Films were then deposited by spin-coating of the $20 \mathrm{wt} \%$ solution $(5 \mathrm{~s}, 1500 \mathrm{rpm})$ onto pre-cleaned silicon substrates or fluorine doped $\mathrm{SnO}_{2}$ (FTO) coated glass sheets (Nippon Sheet Glass, TEC15). The dry hybrid material was subsequently annealed by slow ramping to $130{ }^{\circ} \mathrm{C}$ (180 min linear ramp, $30 \mathrm{~min}$ dwell) and then calcined under conditions as explained in the text. In situ WAXS during the crystallisation process was carried out on a Bruker D8 Advance diffractometer with integrated furnace. A calibrated tube furnace (TSH12, Elite furnaces) was otherwise used for high temperature treatment.

\section{Material characterisation}

WAXS experiments were carried out in situ during heating on a Bruker D8 Advance diffractometer over an angular range of $22^{\circ}-32^{\circ}$ with a step size of $0.0143^{\circ}$ and a total scan time of $5 \mathrm{~min}$. A heating rate of $10^{\circ} \mathrm{C} / \mathrm{min}$ was used unless otherwise stated. Samples were measured 
during crystallisation in air for up to seven hours, representing 84 consecutive X-ray scans. The obtained raw data was corrected for the $\mathrm{CuK}_{\alpha 2}$ radiation by the Bruker software 'EVA'. To obtain the peak broadening caused by the finite size of the anatase crystallites, a Voigt function was fitted to the peaks. The Lorentzian and the Gaussian parts of the Voigt function were determined using the approximation of Olivero and Longbothum [36]. The peak width was then corrected for machine broadening by subtracting (by deconvolution) the broadening of a monocrystalline silicon reference sample. The Debye-Scherrer equation was subsequently employed to obtain a mean crystallite diameter of the peak broadening [37]. As in similar studies, the dimensionless number $K=0.89$ was used as Debye-Scherrer constant [25].

Small-angle X-ray scattering (SAXS) of $\mathrm{CuK}_{\alpha}$ radiation was carried out in transmission geometry at a sample-detector distance of $1.05 \mathrm{~m}$ (Bruker AXS Nanostar). An acquisition time of $7500 \mathrm{~s}$ was used. The scattered intensity was collected with a two-dimensional HiStar gas-filled wire detector (1024 x 1024 channels) and background subtracted. In each case, the scattering patterns were found to be isotropic. Radial scattering intensity profiles were obtained by averaging over the azimuthal angle (from 0 to $360^{\circ}$ ) with $0.005^{\circ}$ increments.

In order to quantify the accessible surface area for dye sensitisation, dye desorption studies were carried out according to studies by to Neale et al. [38]. The absorbance of the resulting light pink solutions was measured with an uv-vis spectrophotometer (HP 8453, wavelength range $190-1100 \mathrm{~nm}, 1 \mathrm{~nm}$ slit width, $0.03 \%$ stray light). The relative amount of dye adsorbed was determined by comparing the peak height of the respective solution with the reference obtained from desorption of the film annealed at $400^{\circ} \mathrm{C}$.

For liquid electrolyte DSC assembly, the samples were sensitised with N719 as described above. Platinisation of the counter electrode was carried out by coating the FTO films with a solution of hexachloroplatinic (IV) acid in anhydrous isopropanol solution $(50 \mathrm{mM})$ followed by heating to $450{ }^{\circ} \mathrm{C}$ for $15 \mathrm{~min}$. The sensitised $\mathrm{TiO}_{2}$ electrodes were washed in acetonitrile, dried and assembled in a sandwich type cell configuration with the platinised counter electrodes. A "hot-melt" $50 \mu \mathrm{m}$ thick surlyn spacer (Dupont) was used to bond the working and counter electrode. A drop of iodine-based liquid electrolyte named "Robust" [39] was subsequently dispensed into a hole in the counter electrode. The cell was filled via vacuum backfilling and sealed with polyimide tape (Dupont). Liquid electrolyte DSCs (active area $0.13 \mathrm{~cm}^{2}$ ) were measured under AM 1.5 simulated sunlight generated by a $300 \mathrm{~W}$ solar simulator (Oriel). The intensity was calibrated with a silicon photo diode (Fraunhofer ISE) and adjusted by a mismatch factor. The currentvoltage measurements were recorded by a Keithley 237 sourcemeter. The incident photon-to-electron conversion efficiency was determined by illumination with a spectrally resolved halogen lamp, which was calibrated by a silicon diode.

Nitrogen physisorption was carried out by Dr. Dominik Eder on a Micromeritics TriStar 
3000 (saturation pressure of $745 \mathrm{mmHg}$ ). Fabrication and characterisation of ss-DSCs were accomplished by Priti Tiwana and Pablo Docampo at the University of Oxford. Impedance spectroscopy was performed by Dr. Burak Ülgüt on a Gamry Reference 600 potentiostat with the EIS300 software package, following a procedure described in reference [40]. See reference [1] for further experimental details.

\subsection{Results and discussion}

The co-assembly of $\mathrm{TiO}_{2}$ into ordered domains on the nanometre scale by the microphase separation of the BCP PI- $b$-PEO is discussed in Chapter 5. The present chapter aims to shed light on the transformation of the initial hybrid film of interdigitated BCP and inorganic material into a crystalline network of mesoporous $\mathrm{TiO}_{2}$. Typically the inorganic material results from sol-gel chemistry, i.e. it is added to the polymer solution in form of a hydrolysed sol, which then complexes with the polar PEO block of the polymer by hydrogen bonding [41, 42]. This means that the $\mathrm{TiO}_{2}$ is initially amorphous and needs thermal activation to nucleate and grow into an anatase network, while the sacrificial organic material is simultaneously oxidised [43]. The temperature stability of the resulting mesoporous morphology is a decisive parameter to achieve highly crystalline networks with good charge carrier properties [26]. Figure 6.1 shows the thermal stability of mesoporous $\mathrm{TiO}_{2}$ derived by PI- $b$-PEO BCP assembly. Structural order after annealing of the films and subsequent removal of the self-assembling $\mathrm{BCP}$ host during crystallisation was probed by SAXS, presented in Figure $6.1 \mathrm{~b}$. The position of the primary scattering peak after annealing at $370{ }^{\circ} \mathrm{C}$ refers to a characteristic length-scale ( $d$-spacing) of $30 \mathrm{~nm}$, shrinking to $27 \mathrm{~nm}$ after complete decomposition of the organic SDA. The first-order peak at $\sim 0.23 \mathrm{~nm}^{-1}$ disappeared only at $740{ }^{\circ} \mathrm{C}$, indicating a loss of structural order at this temperature. Electron microscopy confirmed these observations. Cross-sectional scanning electron microscope (SEM) images of the calcined films in Figure 6.1b and c show that the self-assembled morphology remained intact after crystallisation at temperatures of $430^{\circ} \mathrm{C}(\mathrm{b})$ and $690^{\circ} \mathrm{C}$ (c) for $400 \mathrm{~min}$. The collapse of the pores observed at $740{ }^{\circ} \mathrm{C}$ is evidenced by the SEM cross-section in Figure 6.1d. Thus, the structure imposition of the BCP, organising the inorganic $\mathrm{TiO}_{2}$ sol in a highly ordered network can be seen up to $690^{\circ} \mathrm{C}$.

The overall crystallinity and crystallite size strongly influence charge transport properties of polycrystalline wide band-gap semiconductors and therefore play decisive roles in device applications. Time and temperature of crystal formation are important in controlling these parameters. Figure 6.2a shows wide-angle x-ray scattering (WAXS) patterns of $\mathrm{TiO}_{2}$ films calcined for $400 \mathrm{~min}$ at temperatures between $400^{\circ} \mathrm{C}$ and $700^{\circ} \mathrm{C}$. Up to crystallisation temperatures of $700{ }^{\circ} \mathrm{C}$, all reflections can be indexed to anatase, confirming phase purity for these 

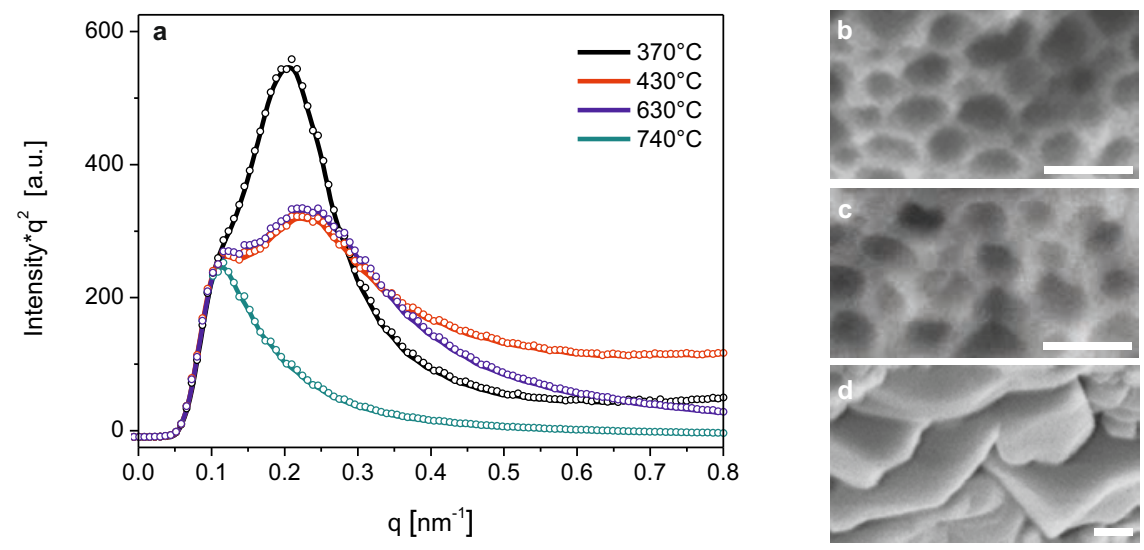

Figure 6.1: Structural stability of BCP-derived morphology. a) Radially integrated SAXS spectra for $\mathrm{BCP}$-assembled $\mathrm{TiO}_{2}$ heat-treated at different temperatures. The spectra are scaled in intensity to overlap in the $0-0.1 \mathrm{~nm}^{-1} q$-range. Cross-sectional SEM images of the nanostructured $\mathrm{TiO}_{2}$ films calcined in air at temperatures of b) $430{ }^{\circ} \mathrm{C}$ and c) $690^{\circ} \mathrm{C}$ for $400 \mathrm{~min}$. d) Crosssectional SEM image of a film crystallised at $740^{\circ} \mathrm{C}$ which resulted in the transformation from anatase to rutile and a subsequent loss of the mesopores. Scale bars represent $100 \mathrm{~nm}$.

calcination conditions [44]. The smaller angular range of Figure 6.2b shows the evolution of the width of the anatase (101) peak with temperature. Diffraction data after crystallisation at $740{ }^{\circ} \mathrm{C}$ is consistent with the coexistence of $\mathrm{TiO}_{2}$ rutile, evidenced by the appearance of the rutile (110) peak at $27.3^{\circ}$. This is line with SAXS data shown in Figure 6.1a and suggests that the structural collapse at $740{ }^{\circ} \mathrm{C}$ is caused by the stresses involved with the lattice reconstruction from anatase to rutile. Debye Scherrer analysis of the full width at half maximum (FWHM) of the (101) peak was used to define the mean crystallite size of the polycrystalline assembly, shown in Figure 6.2c. The final crystallite size was found to be independent of the heating rate in the range of $1-10^{\circ} \mathrm{C} / \mathrm{min}$.

To investigate the time required for completion of the crystallisation process, the crystallite growth was monitored in situ during the calcination process by WAXS. The observed diffraction range for the scattering angle $2 \theta$ between $22^{\circ}$ and $32^{\circ}$ contains the anatase (101) peak, which was used for this analysis. A heterogeneous distribution of crystal sizes during the early stages of crystallisation was clearly observable by electron microscopy. This may be the reason for the poor fitting of the peak width at short crystallisation times. Following the development of the peak area with time and temperature is a more robust measure for the evolution of the crystallite size and was therefore used to follow the kinetics of the crystallisation process. A typical in situ scan of $\mathrm{TiO}_{2}$ during calcination at $530^{\circ} \mathrm{C}$ is shown in Figure $6.2 \mathrm{~d}$, indicating completion of the crystallisation process after about $100 \mathrm{~min}$. The evolution of the overall crystallinity with time was determined by comparing the area of the anatase 
peak with the final peak area after $400 \mathrm{~min}$, which was defined as $100 \%$ crystallinity. The result of this analysis is shown in Figure 6.2e, where the time required to reach values of the overall crystallinity of 50\%-98\% is plotted for a range of temperatures. While near-complete crystallinity $(98 \%)$ was obtained after $100-150 \mathrm{~min}$ for temperatures between $530{ }^{\circ} \mathrm{C}$ and $690^{\circ} \mathrm{C}$, much longer times were needed for temperatures below $500^{\circ} \mathrm{C}$. In addition, the careful investigation of all WAXS samples in reflection (Figure 6.2a and b) and transmission (see supporting information in reference [1]) showed no sign of an amorphous hump. These results are in line with a study by Fattakhova et al. reporting the rapid disappearance of the amorphous signal for crystallisation temperatures between $550{ }^{\circ} \mathrm{C}$ and $650{ }^{\circ} \mathrm{C}$ [26]. It is therefore reasonable to conclude that the amorphous content in all investigated samples was very low when crystallised above $400{ }^{\circ} \mathrm{C}$ for several hours. The effect of the polymer template, spatially confining the crystallisation process, is clearly observable in Figure 6.2f. Here, the mean crystallite size was determined for a variation of the mixing ratio of inorganic and organic material in the synthesis. Lower inorganic loading hereby corresponds to thinner walls, leading to a decrease in crystallite size by $\sim 30 \%$, as seen in Figure 6.2f. In the absence of the organic structure-directing agent, much larger crystals $(>23 \mathrm{~nm}$ ) were observed. 

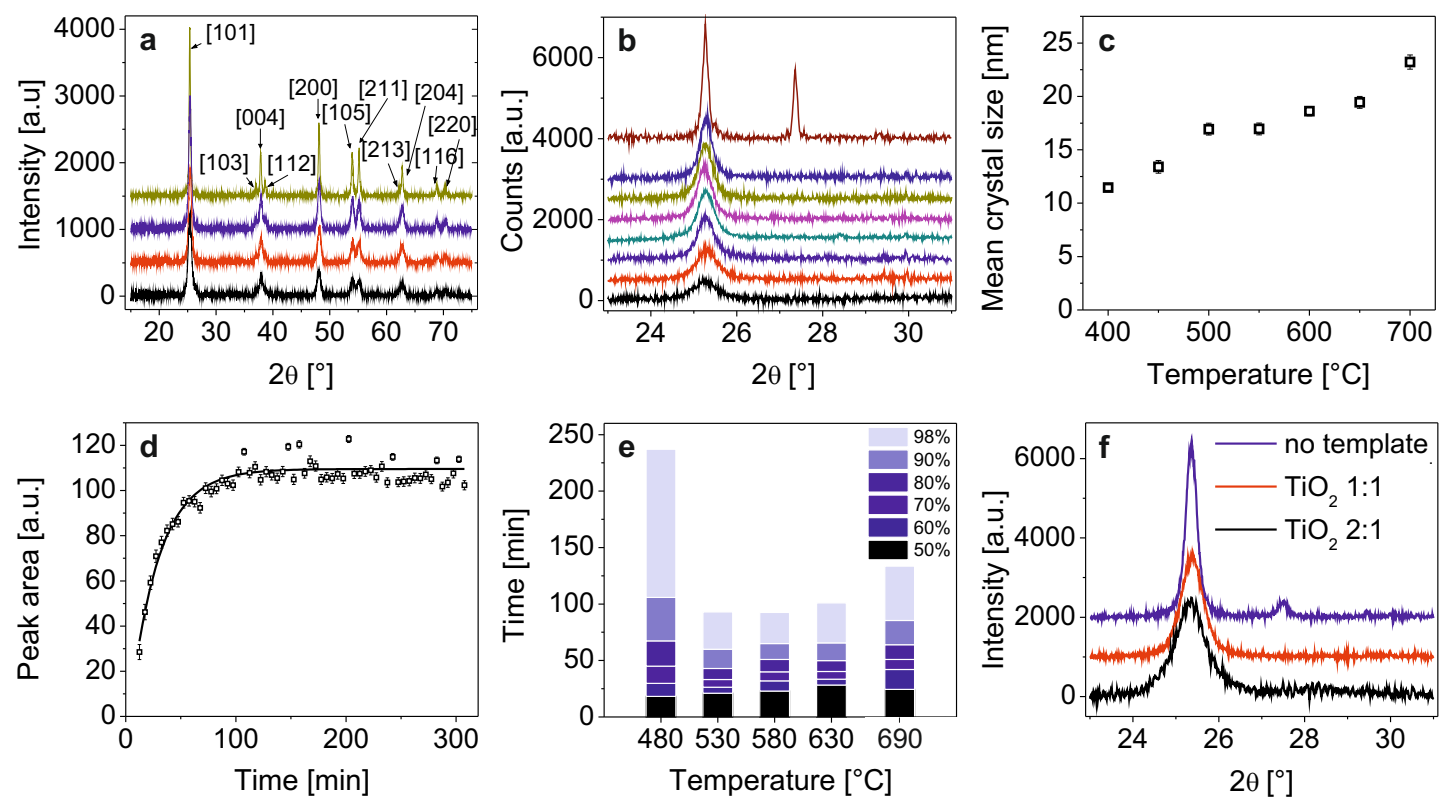

Figure 6.2: Crystallisation of $\mathbf{T i O}_{2}$ anatase crystals in $\mathbf{B C P}$-derived confinement. a) Wide angle x-ray diffraction scans of mesoporous films crystallised at $400^{\circ} \mathrm{C}, 500^{\circ} \mathrm{C}, 600^{\circ} \mathrm{C}$ and $700^{\circ} \mathrm{C}$ (bottom to top). b) Anatase (101) crystal peak as a function of crystallisation temperature after calcination for $400 \mathrm{~min}$. From bottom to top: $400^{\circ} \mathrm{C}$ to $700^{\circ} \mathrm{C}$ in $50{ }^{\circ} \mathrm{C}$ increments (structurally intact) and $740{ }^{\circ} \mathrm{C}$ (collapsed structure). c) Debye-Scherrer mean crystallite sizes after crystallisation for $400 \mathrm{~min}$ as a function of temperature. d) Evolution of crystallinity by monitoring the peak area as a function of time at $530^{\circ} \mathrm{C}$. e) Corresponding evolution of overall crystallinity with time for a range of temperatures. f) Dependence of anatase (101) peak on BCP-dictated confinement. From bottom to top; black: mass ratio $\mathrm{TiO}_{2}: \mathrm{BCP} 1: 1$ (mean crystallite size: $9.5 \mathrm{~nm}$ ); red: 2:1 $(14.7 \mathrm{~nm})$; blue: sol only $(23.6 \mathrm{~nm})$.

High resolution transmission electron microscopy (HRTEM) images of samples calcined at $400^{\circ} \mathrm{C}$ and $650^{\circ} \mathrm{C}$ are shown in Figure 6.3a and b, respectively. After crystallisation at $400^{\circ} \mathrm{C}$ for $400 \mathrm{~min}$, the specimen exhibited a heterogeneous assembly of of $10 \pm 5 \mathrm{~nm}$-sized crystallites. Increasing the temperature to $650^{\circ} \mathrm{C}$, led to larger and more irregularly shaped crystals of around $20 \mathrm{~nm}$ in size, which probably arose from the coalescence of smaller grains. These results are consistent with the x-ray diffraction data analysis. The schematics in Figure $6.3 \mathrm{c}$ and d illustrate crystal growth in BCP-derived confinement at low and high temperatures, respectively. After calcination at $400{ }^{\circ} \mathrm{C}$, dimensions of the crystals are generally smaller than the average width of the $\mathrm{TiO}_{2}$ walls in the network, which is around $15 \mathrm{~nm}$. As the crystals are randomly orientated, growth occurs in this regime isotropically. At higher temperatures crystal growth within the spatial constraints can only proceed anisotropically. This is in line with the observed shape of the crystallites after calcination at $650{ }^{\circ} \mathrm{C}$ (see Figure $6.3 \mathrm{~b}$ ).

The aim of this study was to investigate the interplay between crystallisation of $\mathrm{TiO}_{2}$ in $\mathrm{BCP}$-derived confinement and the corresponding performance of the films in DSC devices. 

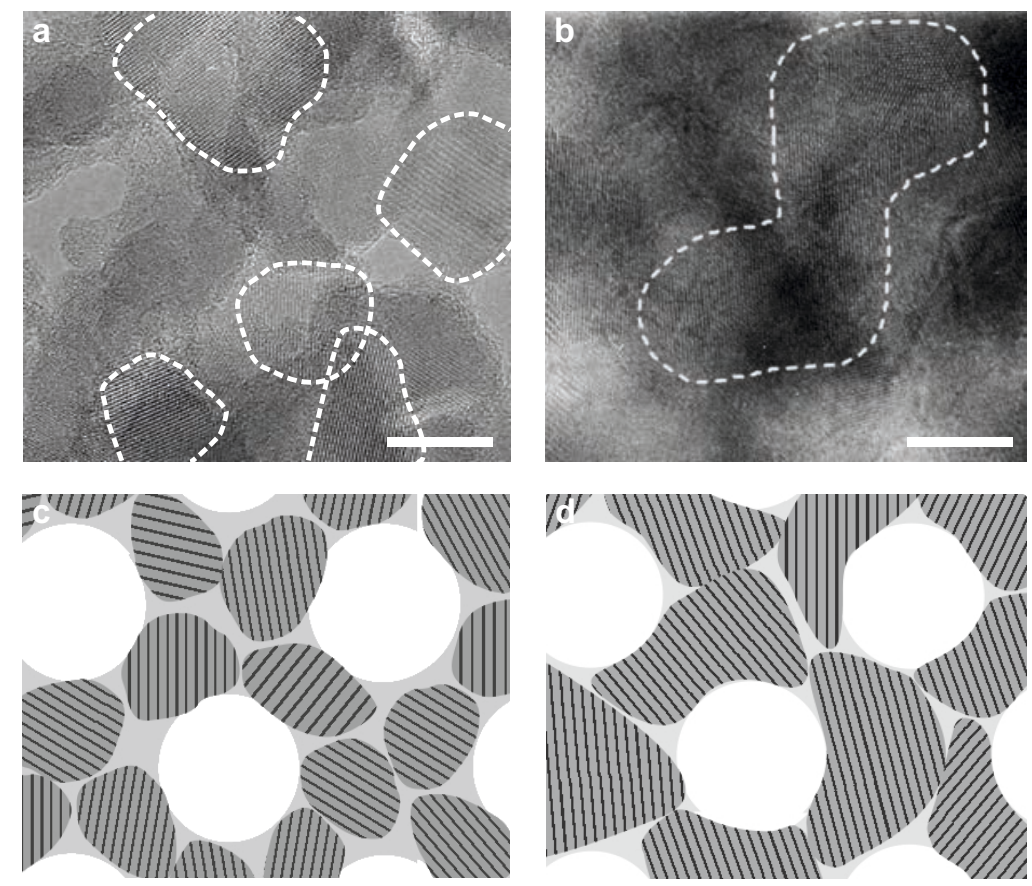

Figure 6.3: Crystallite assembly within the mesoporous network. High resolution transmission electron microscopy (HRTEM) images of samples crystallised at a) $400^{\circ} \mathrm{C}$ and b) $650^{\circ} \mathrm{C}$. White dotted lines are superimposed on the images to visualise individual crystallites. a) Sample calcined at $400^{\circ} \mathrm{C}$ : small, randomly oriented crystals significantly smaller than the length scale of the porous matrix are observed.b) Sample calcined at $650{ }^{\circ} \mathrm{C}$ : crystal size exceeds the length scale of the confinement. Anisotropic growth within the network is likely. Scale bars represent $5 \mathrm{~nm}$. c) d) Schematic illustrating crystallite size and orientation in BCP-derived networks at c) $400^{\circ} \mathrm{C}$ and d) $650^{\circ} \mathrm{C}$.

The development of the accessible pores and surface area with temperature are therefore crucial parameters. Figure 6.4a shows nitrogen adsorption-desorption isotherms of films calcined at $400^{\circ} \mathrm{C}$ and $650{ }^{\circ} \mathrm{C}$, which were subsequently scraped off the substrate to reveal a powder that resembled the morphology of the film. Fitting of the Brunauer, Emmett \& Teller (BET) model in the linear regime of the adsorption-desorption isotherm $\left(0.03<p / p_{0}<0.25\right)$ reveals a reduction in surface area of more than $60 \%$, from $42.0 \mathrm{~m}^{2} / \mathrm{g}$ at $400{ }^{\circ} \mathrm{C}$ to $16.7 \mathrm{~m}^{2} / \mathrm{g}$ for $650{ }^{\circ} \mathrm{C}$ [45]. A simple geometrical consideration, based on SEM image analysis, of a micellar structure with pores of $22 \mathrm{~nm}$ in diameter and a $\mathrm{TiO}_{2}$ wall thickness of $15 \mathrm{~nm}$, leads to a prediction of a surface area of around $20 \mathrm{~m}^{2} / \mathrm{g}$, similar to the value found for the sample calcined at $650{ }^{\circ} \mathrm{C}$. The substantially increased specific surface area of samples calcined at $400{ }^{\circ} \mathrm{C}$ must therefore stem from an additional nanoporosity within the $\mathrm{TiO}_{2}$ structure on smaller length scales than resolvable by SEM or SAXS. Figure $6.4 \mathrm{~b}$ shows the pore distribution for both calcination temperatures as derived from the capillary condensation regime of the isotherm $\left(p / p_{0}>0.4\right)$, following the Barrett, Joyner \& Halenda method (BJH) [46]. The pore 
size distribution is in line with the assumption that nanoporosity arises from the assembly of smaller crystallites within the matrix. This represents a significant contribution to the overall surface area for lower crystallisation temperatures. At higher temperatures the crystallites grow anisotropically within the confined structure and sinter together. At this stage only the flat walls of the BCP-defined mesostructure contribute to the exposed surface area, while the additional degree of nanoporosity within the walls is lost. Due to the limited sample quantity obtainable from thin films, these results should only be seen as a qualitative indication. Similar micellar bulk morphologies, characterised in larger quantities, typically yielded a surface area of $\sim 82 \mathrm{~m}^{2} / \mathrm{g}$ when calcined at $400{ }^{\circ} \mathrm{C}$ and $\sim 64 \mathrm{~m}^{2} / \mathrm{g}$ after crystallisation at $500{ }^{\circ} \mathrm{C}$. Moreover, the $\mathrm{BJH}$ model is disputed for severely underestimating the size of small to medium mesopores while delivering a rather broad size distribution of medium to large mesopores [47].

A more direct measure for surface accessibility is the amount of dye that is chemisorbed as a monolayer onto the $\mathrm{TiO}_{2}$ surface [38]. This can be quantified by subsequent desorption of the dye. Measuring the absorbance of the desorbed solution thus allows to quantify the number of molecules chemisorbed per unit volume. The relative amounts normalised to the value at $400{ }^{\circ} \mathrm{C}$ are compared in Figure $6.4 \mathrm{c}$ for different crystallisation temperatures. The results suggest that the specific accessible surface area only varies weakly in the range between $400^{\circ} \mathrm{C}$ and $550^{\circ} \mathrm{C}$, but drops off for the highest calcination temperatures. This technique also takes into account steric hindrances for surface accessibility, which are associated to the spatial dimensions of the $\mathrm{N} 719$ dye molecule $\left(\mathrm{C}_{58} \mathrm{H}_{86} \mathrm{~N}_{8} \mathrm{O}_{8} \mathrm{RuS} \mathrm{S}_{2}\right)$ compared to $\mathrm{N}_{2}$.

Crystallinity and crystallite size distribution are important measures but it is ultimately their link to the electron conductivity, i.e. the resistivity of the mesoporous film, which de-
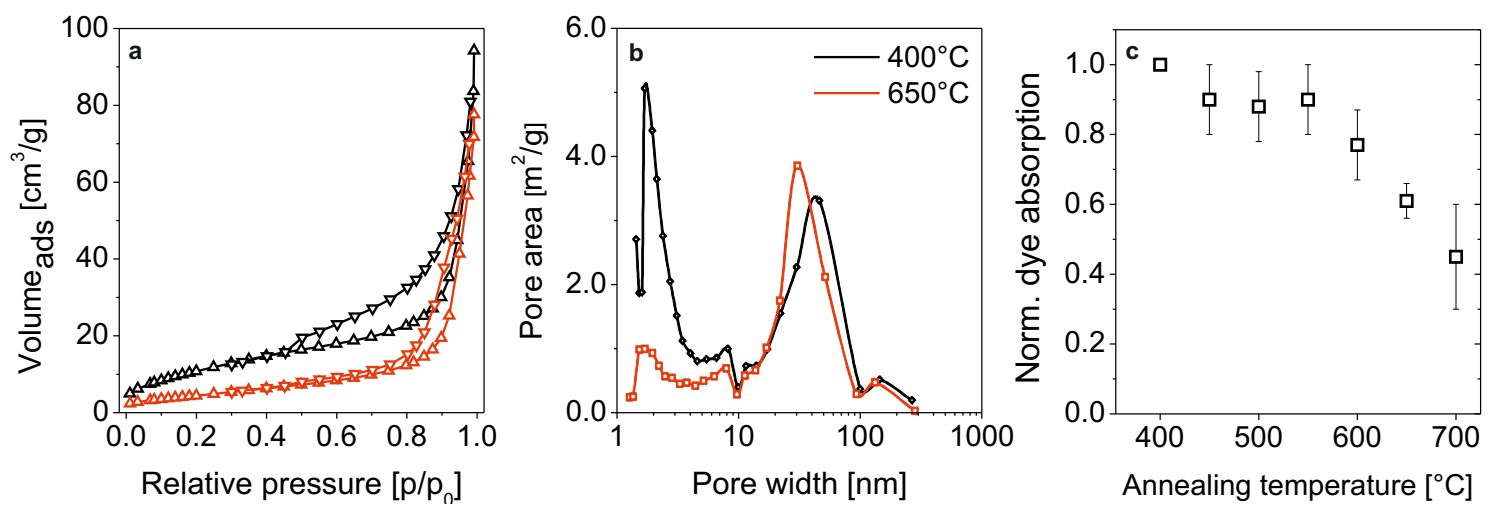

Figure 6.4: Surface area vs. calcination temperature. a) - b) Nitrogen physisorption of films calcined at $400^{\circ} \mathrm{C}$ and $650^{\circ} \mathrm{C}$. a) Adsorption-desorption isotherms for samples calcined at $400^{\circ} \mathrm{C}$ and $650^{\circ} \mathrm{C}$. b) Pore size distribution of mesoporous materials as derived by capillary condensation, following the $\mathrm{BJH}$ method [46]. c) Amount of dye adsorbed per unit area, relative the reference film calcined at $400^{\circ} \mathrm{C}$. 
termines its performance as a photoanode for DSCs. This was characterised by impedance spectroscopy measurements on complete, liquid electrolyte-based devices. The obtained data was subsequently fitted to a transmission line equivalent circuit model adapted from reference [40]. Increasing the calcination temperature from $400^{\circ} \mathrm{C}$ to $650{ }^{\circ} \mathrm{C}$ reduced the resistivity by over a factor of 3 . In contrast, the sheet resistance of the bare FTO substrate increased slightly over this temperature range. These observations are in line with a recent study by Janek and coworkers who compared the photoelectrochemical properties of sol-gel-derived and nanoparticle-based electrodes. They associated enhanced electronic connectivity to the superior performance of sol-gel-based materials [48]. The results of increasing conductivity and decreasing dye loading with temperature give rise to a dilemma for choosing the ideal $\mathrm{TiO}_{2}$ sintering temperature. High temperature calcination leads to large crystallite sizes and therefore a coarser anatase network with a small number of grain boundaries per unit volume. Enhanced charge carrier transport comes at the expense of a reduced surface area, leading to a reduction in dye adsorption and therefore to a decrease in charge generation per unit volume.

This trade-off was further explored in liquid electrolyte and ss-DSCs. For liquid electrolyte DSCs, $\mathrm{TiO}_{2}$ films were crystallised at temperatures ranging from $400{ }^{\circ} \mathrm{C}$ to $650^{\circ} \mathrm{C}$ and subsequently assembled using the dye N719. In this study, $900 \mathrm{~nm}$ thick anatase films were used to avoid the formation of cracks that typically occurs for thicker films. While devices calcined at $400^{\circ} \mathrm{C}$ and $500{ }^{\circ} \mathrm{C}$ had very similar incident photon-to-electron conversion efficiencies (IPCEs) with maxima of $22.0 \%$ and $22.6 \%$, a calcination temperature of $650{ }^{\circ} \mathrm{C}$ resulted in a reduction of the IPCE $_{\max }$ to $18.6 \%$. This decrease in IPCE most likely correlates with the reduced dye loading shown in Figure 6.4c. The corresponding current-voltage $(J-V)$ curves are presented in Figure $6.5 \mathrm{~b}$. While all devices had comparable open-circuit voltages $V_{\mathrm{oc}}$, their short-circuit current $J_{\mathrm{sc}}$ decreased with increasing anatase crystallisation temperature. The related power conversion efficiencies $\eta$ were $2.3 \%, 2.0 \%$ and $1.5 \%$ for $400{ }^{\circ} \mathrm{C}, 500{ }^{\circ} \mathrm{C}$ and $650^{\circ} \mathrm{C}$, respectively. The decrease in $J_{\text {sc }}$ and $\eta$ follows the trend in dye-adsorption and IPCE. While the values for the anatase calcination temperatures of $400{ }^{\circ} \mathrm{C}$ and $500{ }^{\circ} \mathrm{C}$ are comparable, reflecting the plateau in Figure $6.4 \mathrm{c}$, the reduced values for the $650{ }^{\circ} \mathrm{C}$ data-set correlates with the drop-off in specific surface area for this temperature. This result is not surprising. In iodine-based liquid electrolyte cells, charge recombination is not believed to be a limiting factor as it occurs via a slow two-electron process [49]. Therefore, the increased $\mathrm{TiO}_{2}$ conductivity does not outweigh the loss in specific surface area for this cell type. The situation is very different for ss-DSCs. To this end, Figure $6.5 \mathrm{c}$ shows the $J-V$ curves of ss-DSCs, employing the D102 dye and spiro-OMeTAD as hole-conducting medium. 400 nm-thick $\mathrm{TiO}_{2}$ films were used. An overview of the characteristic photovoltaic device parameters is shown in Table 6.1. Increasing the sintering temperature from $350{ }^{\circ} \mathrm{C}$ to $650^{\circ} \mathrm{C}$ resulted in a continuous 

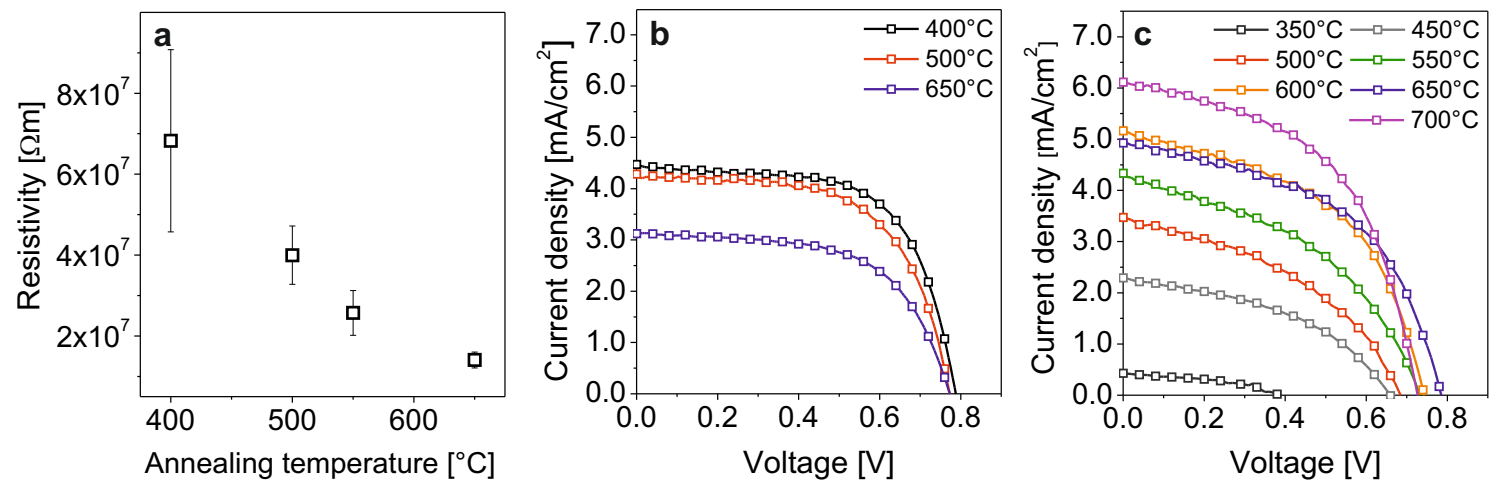

Figure 6.5: Effects of crystallinity on device performance. a) Resistivity determined by impedance spectroscopy of $900 \mathrm{~nm}$ thick films calcined at $400^{\circ} \mathrm{C}, 500^{\circ} \mathrm{C}$ and $650^{\circ} \mathrm{C}$. b) Currentvoltage characteristics for liquid electrolyte DSC devices calcined at $400^{\circ} \mathrm{C}, 500^{\circ} \mathrm{C}$ and $650^{\circ} \mathrm{C}$ (dye N719). c) ss-DSC performance of samples crystallised at $350{ }^{\circ} \mathrm{C}$ to $700^{\circ} \mathrm{C}$. Liquid and solid state devices were characterised under standardised AM 1.5 illumination of $100 \mathrm{~W} / \mathrm{cm}^{2}$.

increase in all relevant performance parameters $\left(V_{\mathrm{oc}}, J_{\mathrm{sc}}, \mathrm{FF}\right.$ and $\left.\eta\right)$. The decrease in $V_{\mathrm{oc}}$ for samples crystallised at $700{ }^{\circ} \mathrm{C}$ might be partially due to the fact that the sheet resistance of the FTO doubled to $40 \Omega / \square$ at these high temperatures. Nevertheless, the power conversion efficiency increased from $0.78 \%$ for $450{ }^{\circ} \mathrm{C}$ to $2.48 \%$ for samples crystallised at $700{ }^{\circ} \mathrm{C}$. Since the specific surface area and dye loading are greatly reduced for the highest anatase crystallisation temperature, the results imply that the ss-DSCs strongly benefit from the increased $\mathrm{TiO}_{2}$ conductivity. While earlier studies reported the relevance of crystallinity and crystal purity of mesoporous networks, it is important to note that the crystallite size is another important parameter to improve photoanode performance in devices, where the efficiency is limited by charge recombination [26]. This effect is expected to be even more pronounced in thicker devices (see Chapter 7). In contrast to conventional approaches, $\mathrm{TiO}_{2}$ films were not post-treated with $\mathrm{TiCl}_{4}$, which further underlines the quality of the anatase produced by this route [50]. 
Table 6.1: Photovoltaic device performance parameters

\begin{tabular}{lcccc}
\hline Calcination temperature & $\begin{array}{c}\mathrm{J} \\
{\left[\mathrm{mA} / \mathrm{cm}^{2}\right]}\end{array}$ & $\begin{array}{c}\mathrm{V} \\
{[\mathrm{V}]}\end{array}$ & $\mathrm{FF}$ & $\begin{array}{c}\eta \\
{[\%]}\end{array}$ \\
\hline solid state DSC & & & & \\
$350^{\circ} \mathrm{C}$ & 0.4 & 0.40 & 0.07 & 0.1 \\
$450^{\circ} \mathrm{C}$ & 2.4 & 0.78 & 0.48 & 0.8 \\
$500^{\circ} \mathrm{C}$ & 3.6 & 0.73 & 0.47 & 1.3 \\
$550^{\circ} \mathrm{C}$ & 4.5 & 0.75 & 0.49 & 1.6 \\
$600^{\circ} \mathrm{C}$ & 5.2 & 0.77 & 0.52 & 2.1 \\
$650^{\circ} \mathrm{C}$ & 5.0 & 0.81 & 0.53 & 2.1 \\
$700^{\circ} \mathrm{C}$ & 6.1 & 0.74 & 0.55 & 2.5 \\
\hline liquid electrolyte DSC & & & & \\
$400^{\circ} \mathrm{C}$ & 4.4 & 0.79 & 0.65 & 2.3 \\
$500^{\circ} \mathrm{C}$ & 4.2 & 0.77 & 0.62 & 2.0 \\
$650^{\circ} \mathrm{C}$ & 3.1 & 0.77 & 0.58 & 1.5 \\
\hline
\end{tabular}

\subsection{Conclusions}

The aim of this study was the exploration of the interplay of crystallisation temperature and time, anatase crystallite size and purity, mesoscopic pore structure, electric resistivity of the porous networks and performance when incorporated into a dye-sensitised solar cell. The selfassembling BCP PI- $b$-PEO is highly effective in controlling anatase crystallisation in mesoporous networks. It rapidly assembles the $\mathrm{TiO}_{2}$ sol into an ordered spatial confinement that promotes crystallite growth within the matrix dictated by the polymer scaffold. Structural integrity can be maintained up to $700^{\circ} \mathrm{C}$, which results in the formation of relatively large crystallites.

When used in DSCs, the control over anatase crystal parameters afforded by this protocol presents an interesting dilemma: while anatase conductivity is markedly improved by high temperature crystallisation, this comes at the expense of specific surface area of the mesoporous scaffold. For liquid DSCs in which charge carrier recombination is not a limitation, the use of high temperature calcined anatase films therefore has a detrimental effect on device performance. In ss-DSCs, on the other hand, the reduced dye loading of the coarser $\mathrm{TiO}_{2}$ network is outweighed by the improved anatase conductivity, leading to an increase in power conversion efficiency by a factor of 3 for cells employing anatase crystallised at $700{ }^{\circ} \mathrm{C}$, compared to materials made by the previously established route.

\section{Bibliography}

[1] S. Guldin, S. Hüttner, P. Tiwana, M. Orilall, B. Ülgüt, M. Stefik, P. Docampo, M. Kolle, G. Divitini, C. Ducati, S. Redfern, H. Snaith, U. Wiesner, D. Eder, and U. Steiner, "Im- 
proved conductivity in dye-sensitised solar cells through block-copolymer confined $\mathrm{TiO}_{2}$ crystallisation," Energy $\mathcal{E}$ Environmental Science, vol. 4, no. 1, pp. 225-233, 2011.

[2] P. Docampo, S. Guldin, U. Steiner, and H. Snaith, "Charge transport limitations in self-assembled $\mathrm{tio}_{2}$ photoanodes for solid-state dye-sensitized solar cell." manuscript in preparation.

[3] B. O'Regan and M. Grätzel, "A low-cost, high-efficiency solar-cell based on dyesensitized colloidal $\mathrm{TiO}_{2}$ films," Nature, vol. 353, no. 6346, pp. 737-740, 1991.

[4] A. Linsebigler, G. Lu, and J. Yates, "Photocatalysis on $\mathrm{TiO}_{2}$ surfaces - principles, mechanisms, and selected results," Chemical Reviews, vol. 95, no. 3, pp. 735-758, 1995.

[5] T. Brezesinski, J. Wang, J. Polleux, B. Dunn, and S. Tolbert, "Templated nanocrystalbased porous $\mathrm{TiO}_{2}$ films for next-generation electrochemical capacitors.," Journal of The American Chemical Society, vol. 131, no. 5, pp. 1802-9, 2009.

[6] O. Varghese, D. Gong, M. Paulose, K. Ong, and C. Grimes, "Hydrogen sensing using titania nanotubes," Sensors And Actuators B-Chemical, vol. 93, no. 1-3, pp. 338-344, 2003.

[7] P. Bonhote, E. Gogniat, F. Campus, L. Walder, and M. Grätzel, "Nanocrystalline electrochromic displays," Displays, vol. 20, no. 3, pp. 137-144, 1999.

[8] S. Burnside, V. Shklover, C. Barbé, P. Comte, F. Arendse, K. Brooks, and M. Grätzel, "Self-organization of $\mathrm{TiO}_{2}$ nanoparticles in thin films," Chemistry Of Materials, vol. 10, no. 9, pp. 2419-2425, 1998.

[9] H. Tang, K. Prasad, R. Sanjines, P. Schmid, and F. Levy, "Electrical and optical properties of $\mathrm{TiO}_{2}$ anatase thin-films," Journal Of Applied Physics, vol. 75, no. 4, pp. 2042-2047, 1994.

[10] L. Forro, O. Chauvet, D. Emin, L. Zuppiroli, H. Berger, and F. Levy, "High-mobility n-type charge-carriers in large single-crystals of anatase $\left(\mathrm{TiO}_{2}\right)$," Journal Of Applied Physics, vol. 75, no. 1, pp. 633-635, 1994.

[11] T. Dittrich, E. Lebedev, and J. Weidmann, "Electron drift mobility in porous $\mathrm{Ti} O_{2}$ (anatase)," Physica Status Solidi A, vol. 1998, no. 165, pp. R5-R6, 1998.

[12] P. Tiwana, P. Parkinson, M. Johnston, H. Snaith, and L. Herz, "Ultrafast terahertz conductivity dynamics in mesoporous $\mathrm{TiO}_{2}$ : influence of dye sensitization and surface treatment in solid-state dye-sensitized solar cells," Journal Of Physical Chemistry C, vol. 114, no. 2 , pp. 1365-1371, 2010.

[13] P. Tiwana, P. Docampo, M. Johnston, H. Snaith, and L. Herz, "Electron mobility and injection dynamics in mesoporous $\mathrm{ZnO}, \mathrm{SnO}_{2}$, and $\mathrm{TiO}_{2}$ films used in dye-sensitized solar cells," ACS Nano, vol. 5, no. 6, pp. 5158-5166, 2011.

[14] A. Frank, N. Kopidakis, and J. van de Lagemaat, "Electrons in nanostructured $\mathrm{TiO}_{2}$ solar cells: transport, recombination and photovoltaic properties," Coordination Chemistry Reviews, vol. 248, no. 13-14, pp. 1165-1179, 2004.

[15] B. O'Regan and J. Durrant, "Kinetic and energetic paradigms for dye-sensitized solar cells: moving from the ideal to the real.," Accounts of chemical research, vol. 42, no. 11, pp. 1799-808, 2009. 
[16] H. Snaith and L. Schmidt-Mende, "Advances in liquid-electrolyte and solid-state dyesensitized solar cells," Advanced Materials, vol. 19, no. 20, pp. 3187-3200, 2007.

[17] L. Li, M. Krissanasaeranee, S. Pattinson, M. Stefik, U. Wiesner, U. Steiner, and D. Eder, "Enhanced photocatalytic properties in well-ordered mesoporous $\mathrm{WO}_{3}$," Chemical Communications, vol. 46, no. 40, pp. 7620-7622, 2010.

[18] N. Tetreault and M. Grätzel, "Novel nanostructures for next generation dye-sensitized solar cells," Energy E Environmental Science, DOI: 10.1039/C2EE03242B, 2012.

[19] J. Melas-Kyriazi, I.-K. Ding, A. Marchioro, A. Punzi, B. Hardin, G. Burkhard, N. Tetreault, M. Grätzel, J.-E. Moser, and M. McGehee, "The effect of hole transport material pore filling on photovoltaic performance in solid-state dye-sensitized solar cells," Advanced Energy Materials, vol. 1, no. 3, pp. 407-414, 2011.

[20] P. Yang, D. Zhao, D. Margolese, B. Chmelka, and G. Stucky, "Generalized syntheses of large-pore mesoporous metal oxides with semicrystalline frameworks," Nature, vol. 396, no. 6707, pp. 152-155, 1998.

[21] E. Crepaldi, G. Soler-Illia, D. Grosso, F. Cagnol, F. Ribot, and C. Sanchez, "Controlled formation of highly organized mesoporous titania thin films: from mesostructured hybrids to mesoporous nanoanatase $\mathrm{TiO}_{2}$," Journal of The American Chemical Society, vol. 125, no. 32, pp. 9770-9786, 2003.

[22] B. Smarsly, D. Grosso, T. Brezesinski, N. Pinna, C. Boissière, M. Antonietti, and C. Sanchez, "Highly crystalline cubic mesoporous $\mathrm{TiO}_{2}$ with 10 -nm pore diameter made with a new block copolymer template," Chemistry Of Materials, vol. 16, no. 15, pp. 2948-2952, 2004.

[23] M. Zukalová, A. Zukal, L. Kavan, M. Nazeeruddin, P. Liska, and M. Grätzel, “Organized mesoporous $\mathrm{TiO}_{2}$ films exhibiting greatly enhanced performance in dye-sensitized solar cells.," Nano Letters, vol. 5, no. 9, pp. 1789-92, 2005.

[24] E. Crossland, M. Kamperman, M. Nedelcu, C. Ducati, U. Wiesner, D.-M. Smilgies, G. Toombes, M. Hillmyer, S. Ludwigs, U. Steiner, and H. Snaith, "A bicontinuous double gyroid hybrid solar cell,” Nano Letters, vol. 9, no. 8, pp. 2807-2812, 2009.

[25] S. Choi, M. Mamak, S. Speakman, N. Chopra, and G. Ozin, "Evolution of nanocrystallinity in periodic mesoporous anatase thin films," Small, vol. 1, no. 2, pp. 226-232, 2005 .

[26] Fattakhova-Rohlfing, D. and Wark, M. and Brezesinski, T. and Smarsly, B.M. and Rathousky, J., "Highly organized mesoporous $\mathrm{TiO}_{2}$ films with controlled crystallinity: a Li-insertion study," Advanced Functional Materials, vol. 17, no. 1, pp. 123-132, 2007.

[27] J. Kondo and K. Domen, "Crystallization of mesoporous metal oxides," Chemistry Of Materials, vol. 20, no. 3, pp. 835-847, 2008.

[28] J. Szeifert, D. Fattakhova-Rohlfing, D. Georgiadou, V. Kalousek, J. Rathousky, D. Kuang, S. Wenger, S. Zakeeruddin, M. Grätzel, and T. Bein, "“'Brick and Mortar" strategy for the formation of highly crystalline mesoporous titania films from nanocrystalline building blocks," Chemistry Of Materials, vol. 21, no. 7, pp. 1260-1265, 2009. 
[29] K. Frindell, M. Bartl, M. Robinson, G. Bazan, A. Popitsch, and G. Stucky, "Visible and near-IR luminescence via energy transfer in rare earth doped mesoporous titania thin films with nanocrystalline walls," Journal Of Solid State Chemistry, vol. 172, no. 1, pp. 81-88, 2003.

[30] I. Kartini, D. Menzies, D. Blake, J. da Costa, P. Meredith, J. Riches, and G. Lu, "Hydrothermal seeded synthesis of mesoporous titania for application in dye-sensitised solar cells (DSSCs)," Journal Of Materials Chemistry, vol. 14, no. 19, pp. 2917-2921, 2004.

[31] M. Templin, A. Franck, A. DuChesne, H. Leist, Y. Zhang, R. Ulrich, V. Schädler, and U. Wiesner, "Organically modified aluminosilicate mesostructures from block copolymer phases," Science, vol. 278, no. 5344, pp. 1795-1798, 1997.

[32] G. Floudas, R. Ulrich, and U. Wiesner, "Microphase separation in poly(isoprene-bethylene oxide) diblock copolymer melts. I. Phase state and kinetics of the order-to-order transitions," Journal Of Chemical Physics, vol. 110, no. 1, pp. 652-663, 1999.

[33] S. Renker, S. Mahajan, D. Babski, I. Schnell, A. Jain, J. Gutmann, Y. Zhang, S. Gruner, H. Spiess, and U. Wiesner, "Nanostructure and shape control in polymer-ceramic hybrids from poly(ethylene oxide)-block-poly(hexyl methacrylate) and aluminosilicates derived from them," Macromolecular Chemistry and Physics, vol. 205, no. 8, pp. 1021-1030, 2004.

[34] J. Allgaier, A. Poppe, L. Willner, and D. Richter, "Synthesis and characterization of poly[1,4-isoprene-b-(ethylene oxide)] and poly[ethylene-co-propylene-b-(ethylene oxide)] block copolymers," Macromolecules, vol. 30, no. 6, pp. 1582-1586, 1997.

[35] S. Warren, F. Disalvo, and U. Wiesner, "Nanoparticle-tuned assembly and disassembly of mesostructured silica hybrids," Nature Materials, vol. 6, no. 2, pp. 156-U23, 2007.

[36] J. Olivero and R. Longbothum, "Empirical fits to voigt line-width - brief review," Journal Of Quantitative Spectroscopy $\mathcal{E}$ Radiative Transfer, vol. 17, no. 2, pp. 233-236, 1977.

[37] P. Scherrer, "Bestimmung der Gröe und der innerern Struktur von Kolloidteilchen mittels Röntgenstrahlen," Nachrichten von der Gesellschaft der Wissenschaften zu Göttingen, mathematisch-physikalische Klasse, pp. 98-100, 1918.

[38] N. Neale, N. Kopidakis, J. van de Lagemaat, M. Grätzel, and A. Frank, "Effect of a coadsorbent on the performance of dye-sensitized $\mathrm{TiO}_{2}$ solar cells: shielding versus bandedge movement," Journal Of Physical Chemistry B, vol. 109, no. 49, pp. 23183-23189, 2005.

[39] D. Kuang, C. Klein, H. Snaith, J. Moser, R. Humphry-Baker, P. Comte, S. Zakeeruddin, and M. Grätzel, "Ion coordinating sensitizer for high efficiency mesoscopic dyesensitized solar cells: influence of lithium ions on the photovoltaic performance of liquid and solid-state cells," Nano Letters, vol. 6, no. 4, pp. 769-773, 2006.

[40] Q. Wang, S. Ito, M. Grätzel, F. Fabregat-Santiago, I. Mora-Sero, J. Bisquert, T. Bessho, and H. Imai, "Characteristics of high efficiency dye-sensitized solar cells," Journal of Physical Chemistry B, vol. 110, no. 50, pp. 25210-25221, 2006.

[41] S. Bagshaw, E. Prouzet, and T. Pinnavaia, "Templating of mesoporous molecular-sieves by nonionic polyethylene oxide surfactants," Science, vol. 269, no. 5228, pp. 1242-1244, 1995. 
[42] S. Boettcher, J. Fan, C.-K. Tsung, Q. Shi, and G. Stucky, "Harnessing the sol-gel process for the assembly of non-silicate mesostructured oxide materials," Accounts Of Chemical Research, vol. 40, no. 9, pp. 784-792, 2007.

[43] C. Sanchez, C. Boissière, D. Grosso, C. Laberty, and L. Nicole, "Design, synthesis, and properties of inorganic and hybrid thin films having periodically organized nanoporosity," Chemistry Of Materials, vol. 20, no. 3, pp. 682-737, 2008.

[44] International Centre for Diffraction Data, "Anatase: PDF 71-1167." PCPDFWIN database, 1998.

[45] Brunauer, S. and Emmett P.H. and Teller E., "Adsorption of gases in multimolecular layers," Journal Of The American Chemical Society, vol. 60, pp. 309-311, 1938.

[46] E. Barrett, L. Joyner, and P. Halenda, "The determination of pore volume and area distributions in porous substances. 1. Computations from nitrogen isotherms," Journal of The American Chemical Society, vol. 73, no. 1, pp. 373-380, 1951.

[47] S. Lowell, J. Shields, M. Thomas, and M. Thommes, Characterization of porous materials and powders: surface area, pore size and density. Springer, 2004.

[48] P. Hartmann, D.-K. Lee, B. Smarsly, and J. Janek, "Mesoporous $\mathrm{TiO}_{2}$ : comparison of classical sol-gel and nanoparticle based photoelectrodes for the water splitting reaction," ACS Nano, vol. 4, no. 6, pp. 3147-3154, 2010.

[49] N. Duffy, L. Peter, R. Rajapakse, and K. Wijayantha, "Investigation of the kinetics of the back reaction of electrons with tri-iodide in dye-sensitized nanocrystalline photovoltaic cells," Journal Of Physical Chemistry B, vol. 104, no. 38, pp. 8916-8919, 2000.

[50] B. O'Regan, J. Durrant, P. Sommeling, and N. Bakker, "Influence of the $\mathrm{TiCl}_{4}$ treatment on nanocrystalline $\mathrm{TiO}_{2}$ films in dye-sensitized solar cells. 2. Charge density, band edge shifts, and quantification of recombination losses at short circuit," Journal Of Physical Chemistry C, vol. 111, no. 37, pp. 14001-14010, 2007. 



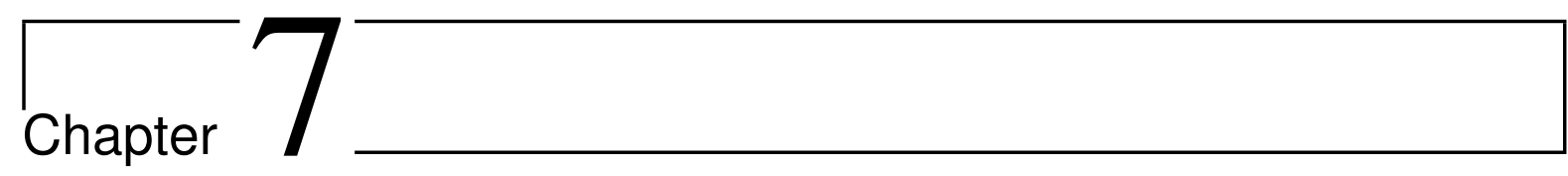

\section{Thin film processing of block copolymer structure-directed inorganic materials}

Published by Stefan Guldin et al. in Small (volume 8, page 432-440, 2012) [1]. See also "Pore-filling of spiro-OMeTAD in solid-state DSCs determined via optical reflectometry", published by Dr. Pablo Docampo in Advanced Functional Materials (volume 22, page 5010-5019, 2012) [2].

\subsection{Introduction}

As outlined in the introductory chapters, mesoporosity, i.e. porosity on the sub-optical length scale, has become an essential feature in materials design for a wide range of applications, such as dye-sensitised solar cells (DSCs) [3], photocatalysis [4], electrochemical capacitors [5], chemical and biochemical sensors [6,7] and electrochromic devices [8]. The high surface area, necessary in these interface sensitive applications, is usually generated by the random close packing of 10-20 nm sized particles, which are obtained by sol-gel chemistry and hydrothermal growth [9]. Although random in pore size and morphological order, these networks have been highly sought after due to the up to 1000-fold increase in surface area, which is a consequence of the sintering of nanometre-sized objects [3]. Nevertheless, a random network of nanoparticles (NPs) lacks detailed control over pore size distribution [9] and exhibits poor charge transport [10]. These characteristics, which are inherent to the electrode architecture, are detrimental for applications with a high demand on electron transport in the semiconductor or mass transport through the porous network [11]. Furthermore the infiltration of a random network of heterogeneously-sized pores is more difficult as the smallest pores act as a barrier and thereby limit effective pore filling over the entire film thickness [12].

One particular example is the solid state dye-sensitised solar cell (ss-DSC, see chapter 3). 
Despite the fact that block copolymer (BCP)-assembled photoelectrode architectures exhibit a much higher control over the pore morphology and offer enhanced charge carrier transport, they still lag behind the maximum conversion efficiencies of NP films [13]. While BCPderived photoelectrode have outperformed NP cells in thin layers (below $1 \mu \mathrm{m}$ ), this performance enhancement could not be extended to thick films $[14,15]$. A fundamental problem is the large volume contraction of the host-guest assembly during the various processing steps. Film shrinkage is induced by the evaporation of residual solvent, the condensation of the inorganic network, the degradation of the polymer and the crystallisation of the $\mathrm{TiO}_{2}$. As a consequence, layers exceeding several hundred nanometres in thickness are subject to crack formation and delamination [16], which limits the thickness of the photoanode and therefore light absorption [17].

Considerable progress has been made in recent years to overcome this issue. Carbone and coworkers proposed a sequential coating and step-by-step calcination to release stresses during the stack build-up [18]. Unfortunately the removal of the polymer during the cyclic calcination step caused a densification of the underlying layers with each subsequent coating. Grosso and coworkers improved this approach by altering the dip coating technique and protecting the pores after each deposition cycle with a sacrificial polymer to maintain the mesoporosity throughout the structure [19]. The group of Li reported the fabrication of several $\mu \mathrm{m}$ thick mesoporous films by the introduction of a synthesis procedure that is based on the deposition of an already gelled solution, followed by a post-deposition treatment in silicone oil [20]. Other approaches include the incorporation of NPs to prevent the formation of microcracks $[21,22]$ and multilayer deposition combined with a sequential rapid calcination process [23]. One appealing concept to reach thicker films is to decouple material manufacturing and film fabrication by synthesising and crystallising the mesoporous $\mathrm{TiO}_{2}$ in the bulk. The as-made material is then processed into a paste and deposited onto a substrate following established routes for NP-based DSCs. Thick films for highly efficient liquid electrolyte DSCs have been reported [24], but the initial self-assembled morphology does not seem to be reflected in the final mesoporous film [25]. Most previous approaches to thick BCP-directed films have been applied to liquid electrolyte DSCs, where demands on a fully continuous and highly conducting $\mathrm{TiO}_{2}$ network are somewhat less important, due to extremely long electron diffusion lengths. Apart from reference [25] the material routes for thick mesoporous films are based on the commercially available Pluronic BCP system (PEO- $b$-PPO- $b$-PEO). As a consequence of its small molecular weight, pore sizes are below $10 \mathrm{~nm}$, and the limited wall thickness does not facilitate complete crystallisation while preserving the mesostructure. Limitations in pore diameter, crystallinity and crystal sizes make this system less ideal for ss-DSCs.

In this chapter, the shrinkage process of BCP-derived inorganic films is studied as a func- 
tion of time and temperature. The in situ investigation permitted the identification of a suitable processing window for a simple and fast treatment protocol that promotes the formation of thick mesoporous films with sufficiently large and continuous pores. The resulting $\mathrm{TiO}_{2}$ coatings were employed as photoanodes in solid state-DSCs and compared to the state-of-the-art nanoparticle film architectures in terms of device efficiency and electronic properties.

\subsection{Experimental}

\section{Material fabrication}

BCP-derived $\mathrm{TiO}_{2}$ photoelectrodes were fabricated as follows: $0.4 \mathrm{~g}$ poly(isoprene-blockethylene oxide) BCP (PI- $b$-PEO) $\left(M_{\mathrm{n}}=34.4 \mathrm{~kg} / \mathrm{mol}, 28 \mathrm{wt} \%\right.$ PEO) was dissolved in an $8 \mathrm{ml}$ azeotrope solvent mixture of toluene (72.84 wt\%, $99.8 \%$ purity) and 1-butanol (27.16 wt\%, $99 \%$ purity). A titanium-containing sol was prepared separately by the addition of $1.54 \mathrm{ml}$ titanium(IV) isopropoxide ( $99.999 \%$ ) to $0.49 \mathrm{ml}$ hydrochloric acid (37\%) under vigorous stirring. 5 min after addition of the titania precursor, the sol was added to the polymer solution and subsequently stirred for a further $30 \mathrm{~min}$. Hybrid films were then deposited from solution by spin coating (1800 rpm, $20 \mathrm{~s}$ ) or doctor blading onto silicon wafers or fluorine doped tin oxide (FTO) coated glass sheets ( $15 \mathrm{Ohm} / \square$, Pilkington) that were previously coated with a compact layer of $\mathrm{TiO}_{2}$. Subsequently the films were exposed to a temperature annealing protocol on a calibrated programmable hotplate $(2000 \mathrm{~W}$, Harry Gestigkeit $\mathrm{GmbH})$. The procedure of film deposition and subsequent annealing was repeated several times (typically three to seven cycles) to achieve the required film thickness. Finally the stacks were calcined at $600{ }^{\circ} \mathrm{C}$ (three hours, heat ramp $5^{\circ} \mathrm{C} / \mathrm{min}$, Cole Parmer, EW-33855-35) to remove the organic material and to crystallise the $\mathrm{TiO}_{2}$.

\section{Material characterisation}

For thermogravimetric analysis (TGA), samples were characterised with a commercial set-up (TA Instruments Q500) in high resolution dynamic mode (ramp $\sim 2{ }^{\circ} \mathrm{C} /$ min unless otherwise stated). An Ocean Optics DHL2000 light source with a combined deuterium halogen lamp served for spectroscopic measurements. A fibre-optic reflection probe (Ocean Optics, R4007-SR) was utilised for sample illumination and signal collection. The reflected signal was detected with an Ocean Optics QE 65000 spectrometer. Optical film thicknesses during annealing processes were acquired from the spectra by least square fitting using a home-built algorithm developed by Prof. J.J. Baumberg, which was implemented by the "Igor Pro" scientific analysis software. Ellipsometric data were taken on a Nanofilm EP3se imaging ellipsome- 
ter and analysed with the instrument software. Fabrication and characterisation of solid state DSCs was carried out by Pablo Docampo (University of Oxford). The particular TGA analysis presented in Figure 7.2 was done by Dr. Dominik Eder, while characterisation was otherwise performed by the author. See reference [1] for ss-DSC device fabrication and characterisation as well as further experimental details.

\subsection{Results and discussion}

As previously discussed, the manufacture of thick porous films derived from a sol-gel/BCP composite is problematic because of the considerable shrinkage during the condensation reaction, polymer removal and $\mathrm{TiO}_{2}$ crystallisation, typically leading to crack formation and delamination. The necessity of a stepwise release of stresses in a layer-by-layer approach becomes clear when investigating the loss in film thickness of an initially $700 \mathrm{~nm}$ thick layer as a function of temperature. Figure 7.1a shows the shrinkage of films derived from a weight ratio of $1: 1$ between the resulting $\mathrm{TiO}_{2}$ after fabrication and the mixed polymer in the initial solution $\left(\mathrm{TiO}_{2} 1: 1\right)$ when exposed overnight to temperatures between $50^{\circ} \mathrm{C}$ and $600{ }^{\circ} \mathrm{C}$. Shrinkage levels off at around $400{ }^{\circ} \mathrm{C}$, after evaporation of residual solvent, condensation of the $\mathrm{TiO}_{2}$ network and complete oxidation of the polymer have led to a decrease in film thickness by $60 \%$. Film shrinkage is significant for all inorganic to organic mixing ratios employed in the course of this study, with $55 \%$ thickness contraction for $\mathrm{TiO}_{2} 3: 1$ and $65 \%$ for $\mathrm{TiO}_{2} 1: 2$, as demonstrated in Figure 7.1b.
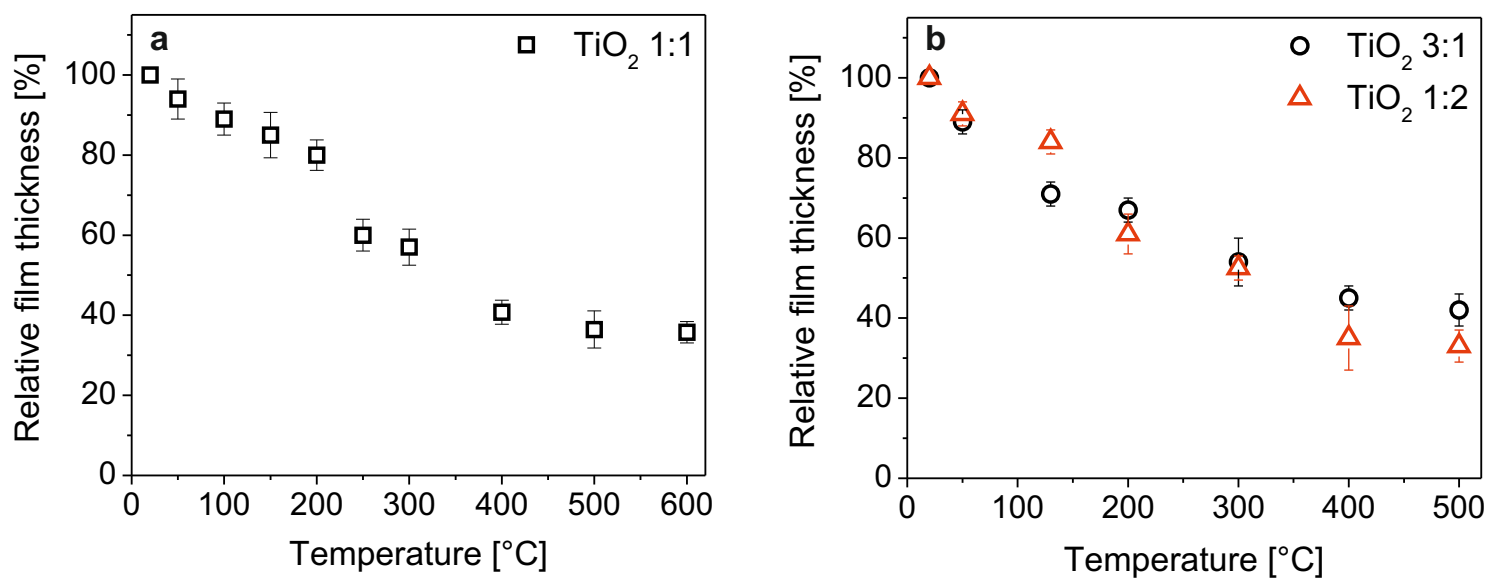

Figure 7.1: Temperature dependent shrinkage in film thickness. a) Ex situ development of the film thickness $h$ as a function of annealing temperature, determined by ellipsometry and profilometry. The films were derived from an inorganic to organic weight ratio of $1: 1 \mathrm{in}$ solution $\left(\mathrm{TiO}_{2}\right.$ 1:1) and annealed overnight at each temperature. b) Film thickness as a function of temperature for hybrid films for weight ratios of 3:1 $\left(\mathrm{TiO}_{2} 3: 1\right)$ and 1:2 $\left(\mathrm{TiO}_{2}\right.$ 1:2). 
An important aspect of this work is revealed by thermogravimetric analysis (TGA), presented in Figure 7.2. Besides the hybrid samples, $\mathrm{BCP}$ and $\mathrm{TiO}_{2}$ sol-gel material were also individually analysed by TGA. An expected development of the temperature dependent weight loss was subsequently calculated by linear combination of the inorganic and organic mass fraction in the hybrid materials. Compared to this anticipated development, samples with an inorganic mixing ratio of $3: 1\left(\mathrm{TiO}_{2} 3: 1\right)$ showed a retardation of the weight loss by $68^{\circ} \mathrm{C}$, whereas a mixing ratio of $1: 2\left(\mathrm{TiO}_{2} 1: 2\right)$ led to a delay of $75^{\circ} \mathrm{C}$. The greatly enhanced temperature resistivity of a hybrid sample compared to the purely inorganic and organic materials can most likely be attributed to the fact that the $\mathrm{TiO}_{2}$ sol complexes with the PEO to form a hybrid matrix, which makes the organic component less susceptible to oxidation.

The resistance of the $\mathrm{PI}-b-\mathrm{PEO} / \mathrm{TiO}_{2}$ hybrid system against high annealing temperatures is of central importance in this study. Earlier work has shown that a layer-by-layer approach can suppress crack formation due to the gradual release of stresses associated with the volume contraction. This is usually achieved by the calcination of the film after every layer deposition, which either leads to a densification of the film structure with subsequent coatings or requires an additional infiltration step to protect the pores $[18,19]$. Both approaches may lead to a discontinuity of the porous network at the interfaces of each individual layer [26].

The aim of this study was therefore to anneal at temperatures that result in significant volume contraction but still allows pore protection by residual organic material. The appropriate annealing temperature is a trade-off between maximising stress release (by annealing at sufficiently high temperatures) and preserving most of the protective organic material (by
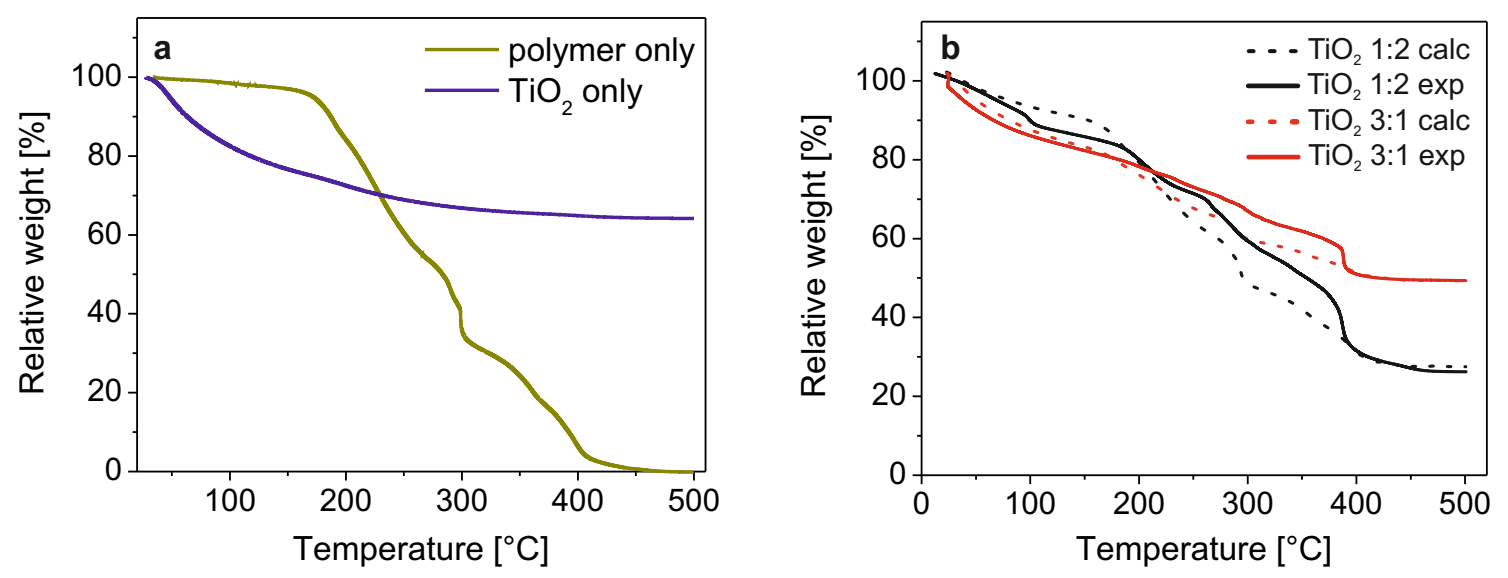

Figure 7.2: Temperature dependent weight loss. a) Weight loss of $\mathrm{BCP}$ (dark yellow) and $\mathrm{TiO}_{2}$ sol (blue) during thermogravimetric analysis (TGA). b) Comparison of expected and measured weight loss by TGA. Dotted lines represent the weighed average derived from TGA results of purely organic and inorganic samples. Solid lines correspond to the measured results for inorganic to organic ratios of 1:2 (black) and 3:1 (red). 
annealing at low enough temperatures). According to the data shown in Figures 7.1 and 7.2, a temperature of $300{ }^{\circ} \mathrm{C}$ is suitable to meet both requirements. Following the temperaturedependent weight loss of the pure $\mathrm{TiO}_{2}$ sol it appears that the condensation of the hydrolysed $\mathrm{Ti}(\mathrm{OH})_{4}$ to $\mathrm{TiO}_{2}$ has completed at this temperature. Film shrinkage has already proceeded to $82 \%, 70 \%$ and $74 \%$ of its overall loss in thickness for the different mixing ratios $\mathrm{TiO}_{2} 3: 1$, $\mathrm{TiO}_{2}$ 1:1 and $\mathrm{TiO}_{2}$ 1:2, respectively. Yet, according to the TGA results, a significant fraction of organic material is still present for finite annealing times at this temperature. The sacrificial material plays a crucial role for the formation of smooth and well-interconnected interfaces in a layer-by-layer stacking since the residual polymers protect the pores of the underlying film during the deposition of the subsequent layer. Furthermore, as the condensation of the inorganic network is more or less complete at $300^{\circ} \mathrm{C}$, partial dissolution of the underlying network is prevented when in contact with the solution during the cyclic film deposition process. Only in a final calcination and crystallisation step above $400{ }^{\circ} \mathrm{C}$ is the organic material fully removed to reveal the porous network. As will be shown in Chapter 8, subsequent layers in a multilayer stack are identical in their optical properties, i.e. of similar porosity and film thickness. This is convincing evidence that in contrast to earlier approaches, the pore size and pore shape stays constant throughout the layers $[18,27]$.

In order to resolve the kinetics of the shrinkage process, the annealing of the films was monitored in situ on a hotplate by collecting their reflection spectra. Figure 7.3 shows contour plots of time-resolved reflection spectra for annealing temperatures of $50{ }^{\circ} \mathrm{C}$ to $300{ }^{\circ} \mathrm{C}$. The reflection intensity, normalised to that of a bare silicon wafer, is represented in a multi-colour scheme. The position of the interference fringes depends on the effective refractive index of the material $n$ and the thickness of the film $h$ (see Chapter 2). A shift of the reflectance maxima and minima to smaller wavelengths therefore corresponds to a reduction of the optical film thickness.

The optical film thickness $n \cdot h$ was subsequently obtained by least square fitting. The corresponding values at each temperature as a function of the annealing time are plotted in Figure 7.4a. Error bars include fitting uncertainties as well as the statistical standard deviation derived from three to five runs. For comparison, results were normalised to the initial film thickness of the samples at room temperature. Complementary ex situ measurements of the individual development of $n$ with temperature are shown in Figure 7.4b. For this purpose, the samples were annealed overnight at the corresponding temperature and subsequently characterised by imaging ellipsometry. These results suggest that $n$ is more or less unaffected by the annealing temperature with a maximum decrease of $\sim 6 \%$ after annealing at $300{ }^{\circ} \mathrm{C}$ overnight. Hence, the development of the optically accessible parameter $n \cdot h$ is a good measure to reveal the development of the film thickness. 

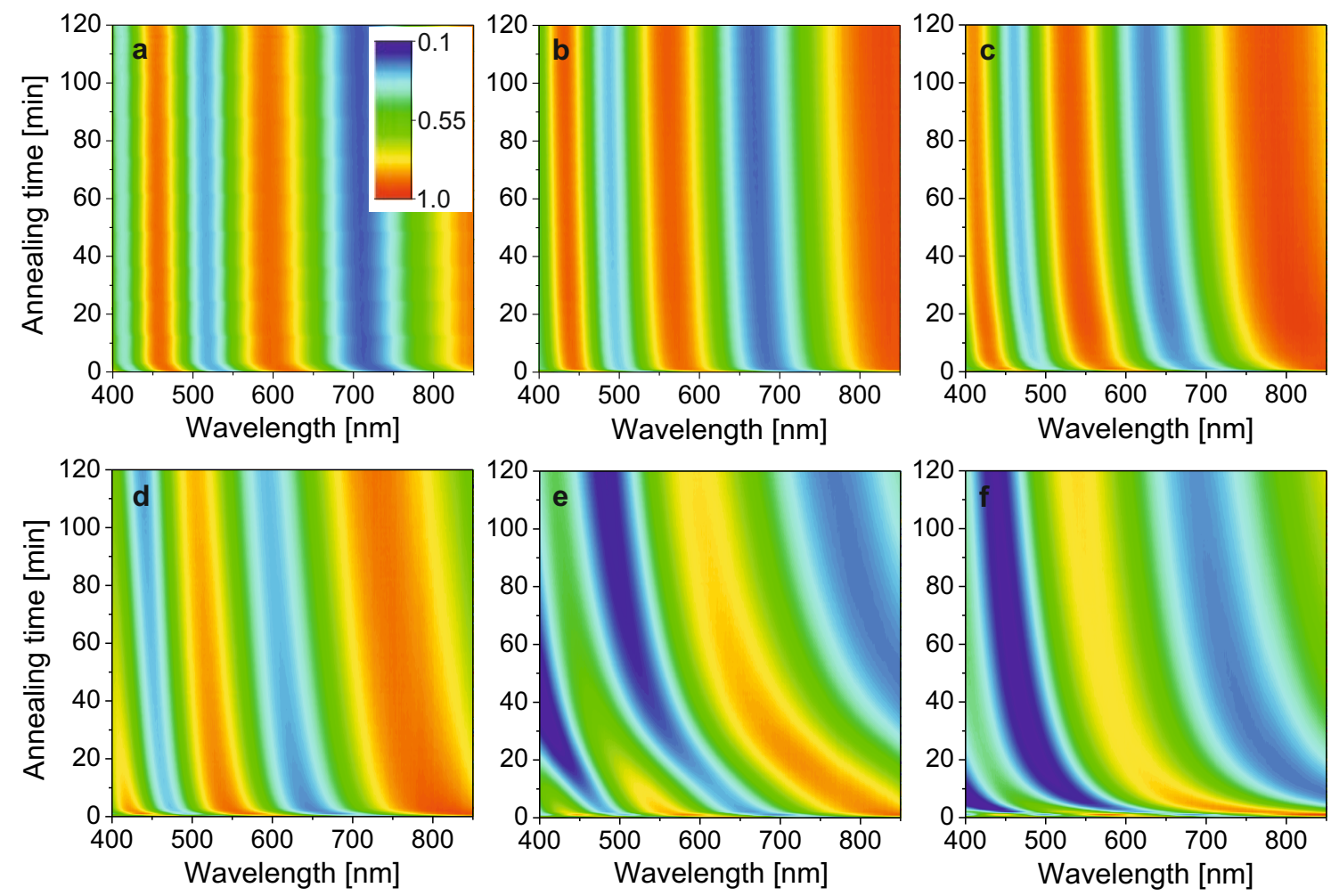

Figure 7.3: In situ monitoring of film shrinkage at constant temperatures. Reflectance spectra of $\sim 700 \mathrm{~nm}$ thick hybrid films during annealing on a hotplate at a) $50^{\circ} \mathrm{C}$, b) $100^{\circ} \mathrm{C}$, c) $150^{\circ} \mathrm{C}$, d) $200{ }^{\circ} \mathrm{C}$, e) $250^{\circ} \mathrm{C}$ and f) $300^{\circ} \mathrm{C}$. The time-resolved reflection intensity is plotted in a multi color scheme shifting from blue to red for low to high intensities. The data is normalised to the reflectivity of a blank silicon wafer. A shrinkage in film thickness corresponds to a shift of the reflectance maxima and minima to smaller wavelengths.
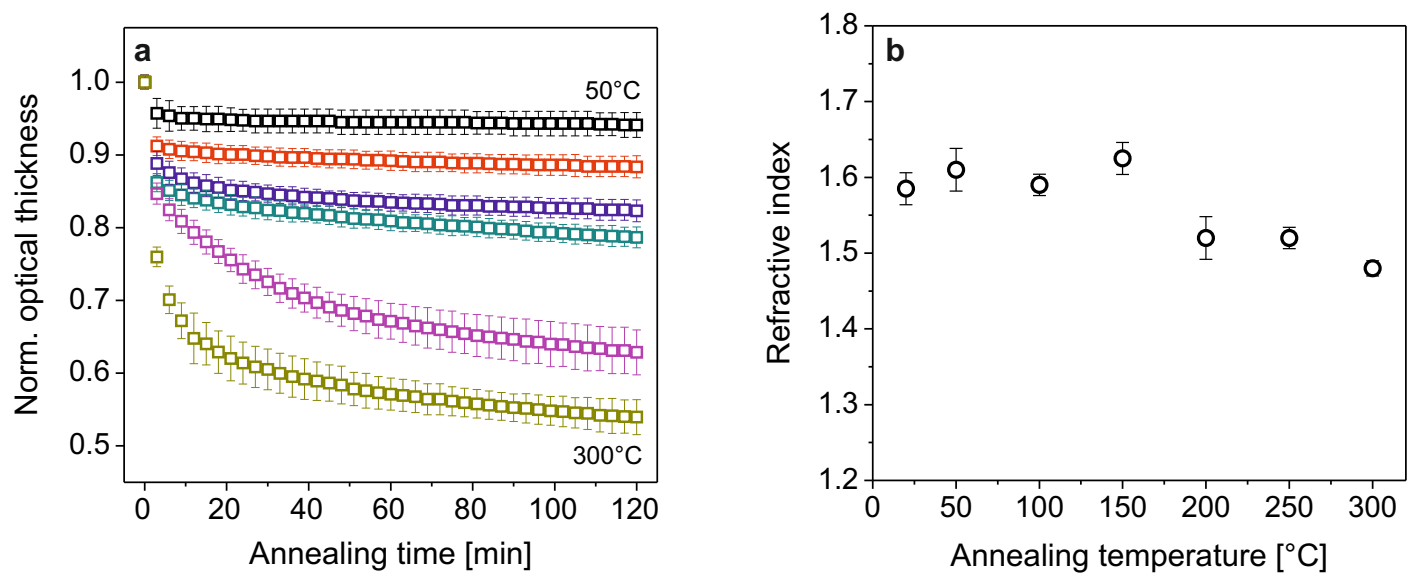

Figure 7.4: Time-resolved development of optical film thickness at constant temperatures. a) Development of the optical film thickness for different annealing temperatures (from top to bottom: $50{ }^{\circ} \mathrm{C}-300^{\circ} \mathrm{C}$ in $50{ }^{\circ} \mathrm{C}$ steps). Thickness values were derived from reflectance spectra by least square fitting. Error bars shown include fitting uncertainties as well as statistical standard deviation derived from three to five runs. b) Refractive index $n$ as a function of annealing temperature determined by imaging ellipsometry. Samples were annealed overnight at each temperature. 
Results from annealing at constant temperatures allowed the establishment of an annealing protocol, which enabled the gradual decrease of thickness with time and temperature, but limited the processing time to a minimum. This protocol consisted of an initial $10 \mathrm{~min}$ dwell time at $50^{\circ} \mathrm{C}$ to evaporate the solvent and promote microphase separation, followed by a linear $45 \mathrm{~min}$ increase in temperature to $300^{\circ} \mathrm{C}$, where the films remained for $5 \mathrm{~min}$ before they were rapidly cooled down. The effect of such a 60 min annealing protocol on film thickness and weight is shown in Figure 7.5. In comparison to static annealing at a fixed temperature (Figure 7.4a), the temperature protocol has the beneficial effect of slowly and gradually reducing the film thickness by $37 \%$. The greatly enhanced film integrity is therefore a direct consequence of the annealing protocol. A complementary TGA measurement of the films subject to this temperature procedure is shown for comparison. After $60 \mathrm{~min}$ of annealing to $300{ }^{\circ} \mathrm{C}$, the films retained $63 \%$ of their initial weight. This compares to a weight of $33 \%$ after removing the residual organic material and crystallising the $\mathrm{TiO}_{2}$ network at $500{ }^{\circ} \mathrm{C}$. A significant amount of organic material is therefore still present at $300{ }^{\circ} \mathrm{C}$ to protect the pores during successive layer deposition.

Figure 7.6 shows a schematic outline of the proposed layer-by-layer preparation route. A solution of PI- $b$-PEO BCP and $\mathrm{TiO}_{2}$ sol in an azeotrope mixture of toluene and 1-butanol is co-deposited on a FTO-covered glass substrate by spin coating, doctor blading or dip-coating.
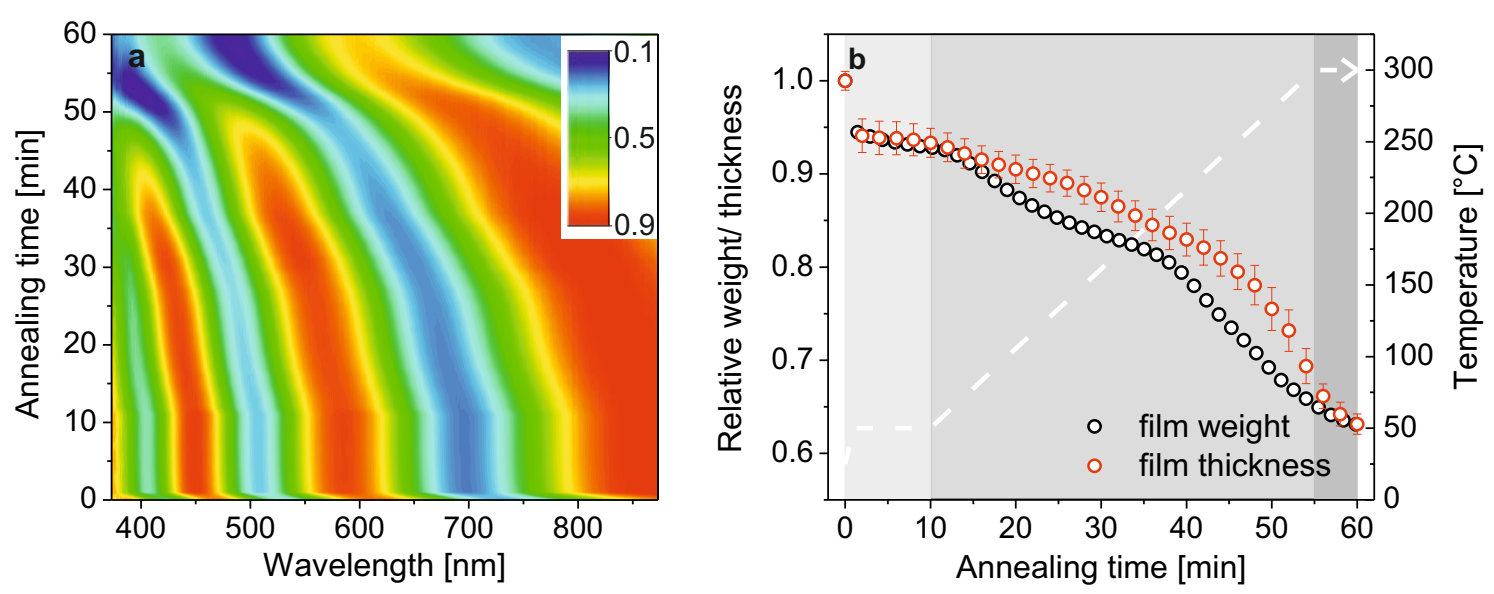

Figure 7.5: Effect of annealing protocol on film shrinkage. a) Optical response of a hybrid film subject to the proposed $60 \mathrm{~min}$ annealing protocol. The time-resolved reflection intensity, normalised to that of a bare silicon wafer, is represented in a multi-colour scheme. b) Corresponding development of the optical film thickness during the temperature treatment as derived by least square fitting of the reflectance spectra. In contrast to annealing at a fixed temperature, shrinkage occurs gradually, leading to a thickness reduction by $37 \%$ (statistical average of five runs). For comparison the weight loss of the films was derived by TGA under the identical heating procedure. The temperature profile of the protocol is shown as a white dashed line. 
Upon evaporation of the solvent, the hybrid material phase separates into nanometre-sized domains, where the $\mathrm{TiO}_{2}$ sol resides in the hydrophilic PEO block to form a matrix that surrounds the pore forming hydrophobic PI block. The use of an azeotrope with a common high boiling point promotes a slow and simultaneous evaporation of hydrophobic and hydrophilic solution components. After coating from solution the film contains residual solvent, and the inorganic material has not yet fully condensed into a $\mathrm{TiO}_{2}$ network. The hybrid film is then subjected to a temperature protocol of $10 \mathrm{~min}$ at $50^{\circ} \mathrm{C}$, followed by a $45 \mathrm{~min}$ linear heating ramp to $300^{\circ} \mathrm{C}$ and a final dwell time of $5 \mathrm{~min}$ at this temperature. The $60 \mathrm{~min}$ annealing procedure typically leads to a thickness shrinkage of $\sim 40 \%$. Best results were achieved when the amount of dry film did not exceed $\sim 700 \mathrm{~nm}$ per layer deposition. A stack is produced by repeating the film deposition and annealing cycles to reach the desired final film thickness in typically three to seven steps. Finally, a high temperature step at $600^{\circ} \mathrm{C}$ for $3 \mathrm{~h}$ removes the organic components and crystallises the $\mathrm{TiO}_{2}$. See experimental section for further details.

This protocol enabled the gradual build-up of a thick film in a layer-by-layer fashion, releasing most of the stresses caused by volume reduction before deposition of the subsequent

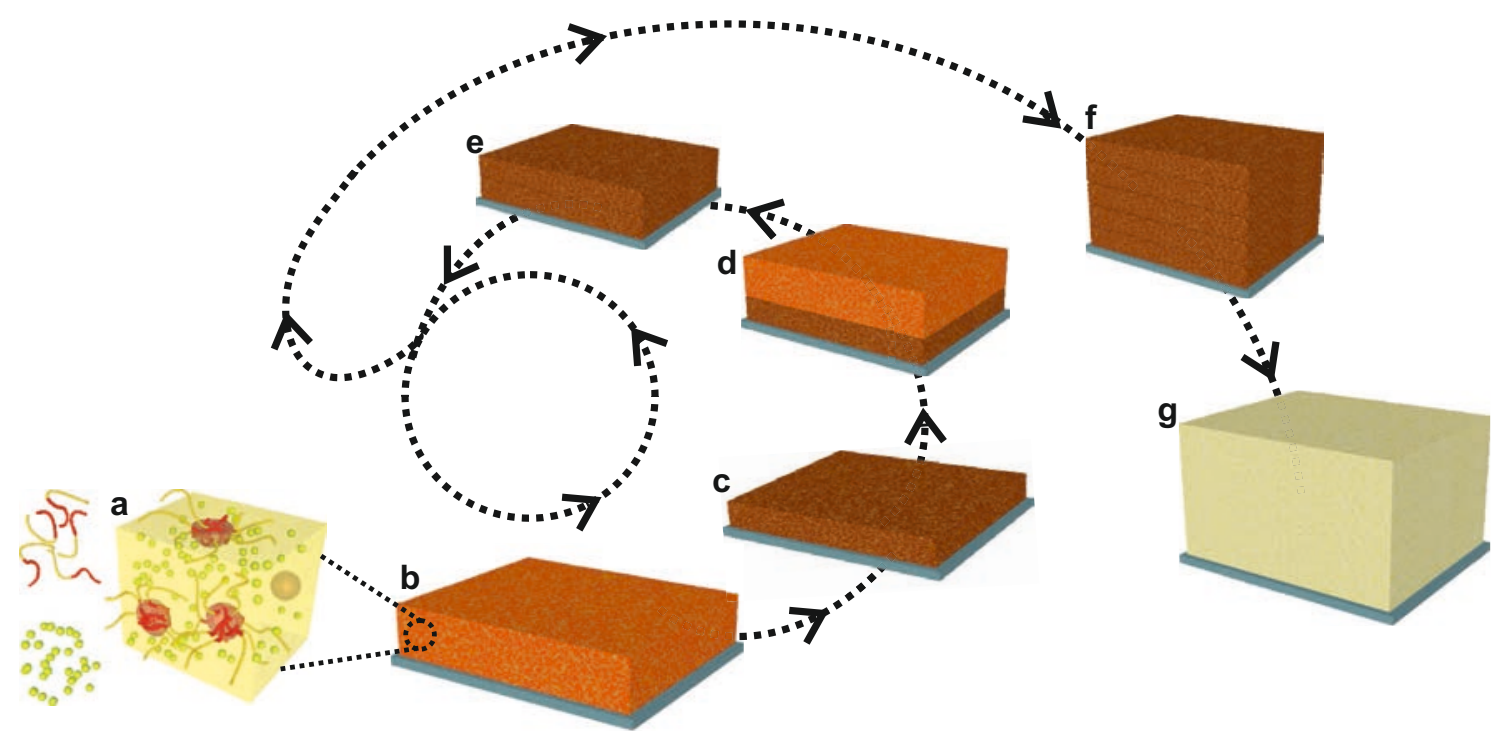

Figure 7.6: Schematic of film fabrication. a) A solution of PI- $b$-PEO BCP and $\mathrm{TiO}_{2}$ sol is codeposited on a FTO-covered glass substrate by spin coating, doctor blading or dip-coating. The inorganic component preferentially resides in the PEO phase and is therefore structure-directed during the self-assembly process of the amphiphilic BCP. b) After deposition from solution, the film contains residual solvent and the inorganic material has not yet fully condensed into a $\mathrm{TiO}_{2}$ network. c) The hybrid film is then subjected to a $60 \mathrm{~min}$ temperature ramp to $300{ }^{\circ} \mathrm{C}$, leading to a thickness shrinkage of $\sim 40 \%$. d) - e) Film deposition and heating are repeated several times to reach $\mathbf{f}$ ) the desired overall film thickness. g) A final high temperature calcination at $600{ }^{\circ} \mathrm{C}$ removes the organic components and crystallises the $\mathrm{TiO}_{2}$ film. 
layer. The beneficial effect of this method is shown in Figure 7.7, where a $2.5 \mu \mathrm{m}$ thick stack consisting of six layers is compared to a $2.5 \mu \mathrm{m}$ thick film made in a single step. Figure $7.7 \mathrm{a}$ displays an optical transmission micrograph shortly after the deposition of a highly concentrated solution to form a $2.5 \mu \mathrm{m}$ thick film. The evaporation of solvents caused the formation of cracks even before temperature annealing. After annealing, calcination and $\mathrm{TiO}_{2}$ crystallisation at high temperatures, the film has broken up into isolated islands of mesoporous $\mathrm{TiO}_{2}$, shown in the scanning electron micrograph in Figure 7.7b. As a consequence, a large area of the substrate (> $40 \%$ ) was left uncovered. In contrast, an equally thick film deposited in six cycles showed no cracks before and after annealing at $600{ }^{\circ} \mathrm{C}$ (Figure $7.7 \mathrm{c}-\mathrm{d}$ ).

The use of the PI- $b$-PEO BCP is particularly attractive because the combination of a high PI-PEO Flory-Huggins interaction parameter with low glass transition temperatures of both blocks and the large degree of polymerisation results in a much more robust and fast phase separation as compared to other BCP systems [28]. Instead of relying on time consuming protocols, the PI- $b$-PEO microphase morphology forms within minutes, allowing fast and reliable multilayer assembly.
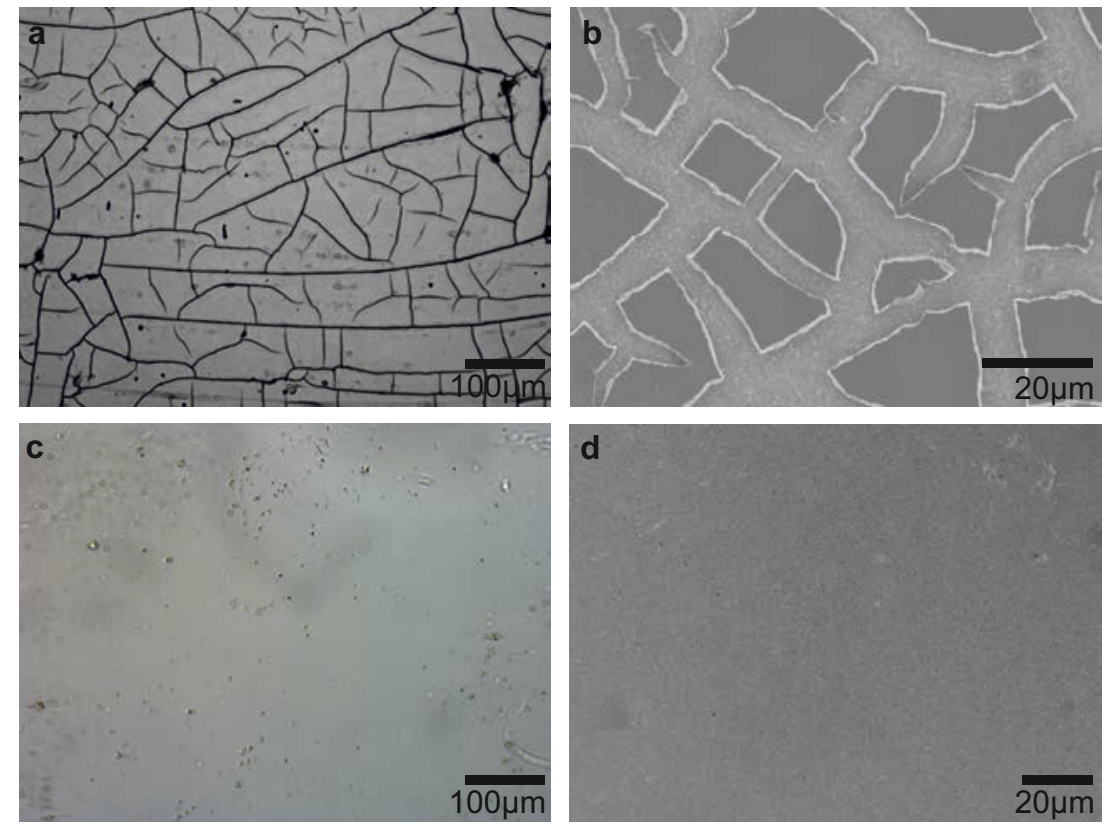

Figure 7.7: Film quality and cracking behaviour. a) - b) A $2.5 \mu \mathrm{m}$ thick film was deposited in a single step. a) Transmission optical micrograph of the hybrid film. Evaporation of solvents led to the formation of cracks even at room temperature. b) SEM top-view image after high temperature treatment. Initial cracks expanded during calcination and $\mathrm{TiO}_{2}$ crystallisation, leaving a large substrate area uncovered (> 40\%). c) - d) Formation of a similarly thick film in 6 cycles. c) Optical micrograph of the hybrid film before and d) SEM image of the $2.5 \mu \mathrm{m}$ thick film after calcination and $\mathrm{TiO}_{2}$ crystallisation. 
The morphology of a $\mathrm{TiO}_{2}$ photoanode fabricated by four deposition and annealing cycles and a final high temperature step is shown in Figure 7.8. Pores are well discernible on the surface of the $1.3 \mu \mathrm{m}$ thick stack, indicating that the pore network is fully accessible from the top. No solid $\mathrm{TiO}_{2}$ overlayer has formed that could impede the infiltration of the network. Figure $7.8 \mathrm{~b}$ shows the cross-section of a fully assembled ss-DSC, after dye sensitisation and infiltration of the network with the hole conductor spiro-OMeTAD.

Thick porous multilayer photoanodes assembled into ss-DSCs exhibited remarkable photovoltaic performance. Figure 7.9a presents the current-voltage characteristics of the best performing device, with a short circuit current $\left(J_{\mathrm{sc}}\right)$ of $8.0 \mathrm{~mA} / \mathrm{cm}^{2}$, an open-circuit potential $\left(V_{\mathrm{oc}}\right)$ of $0.81 \mathrm{~V}$ and a fill factor $(F F)$ of 0.63 , resulting in a power conversion efficiency $(\eta)$ of $4.0 \%$ using the organic dye CN04 [13]. This is a significant improvement over previously reported efficiencies of BCP-assembled ss-DSCs, where $\eta_{\max }=2.5 \%$ [15] and $\eta_{\max }=1.5 \%$ [29]. In both cases, the attainable film thicknesses were limited by cracking and delamination, resulting in insufficient light absorption of the manufactured devices. Devices were also assembled with the indolene based organic dye D102 which is often used to create reliable ss-DSCs. Figure 7.9b shows the best working devices employing this dye. Devices based on BCP-assembled and conventional NP photoanodes reach similar power conversion efficiencies of $\sim 3.6 \%$. While the NP system has a higher value of $J_{\mathrm{sc}}$, the BCP multilayer devices excel in open-circuit voltage and fill factor. Figure 7.9c-d shows an overview of the key photovoltaic device parameters $J_{\mathrm{sc}}, \eta, V_{\mathrm{oc}}$ and $F F$ as a function of the film thickness of the BCP-assembled photoanode (data from 12 devices were averaged for each thickness). A reasonably constant performance was observed for photoanode thicknesses between 1.3 and $2.0 \mu \mathrm{m}$. Devices with
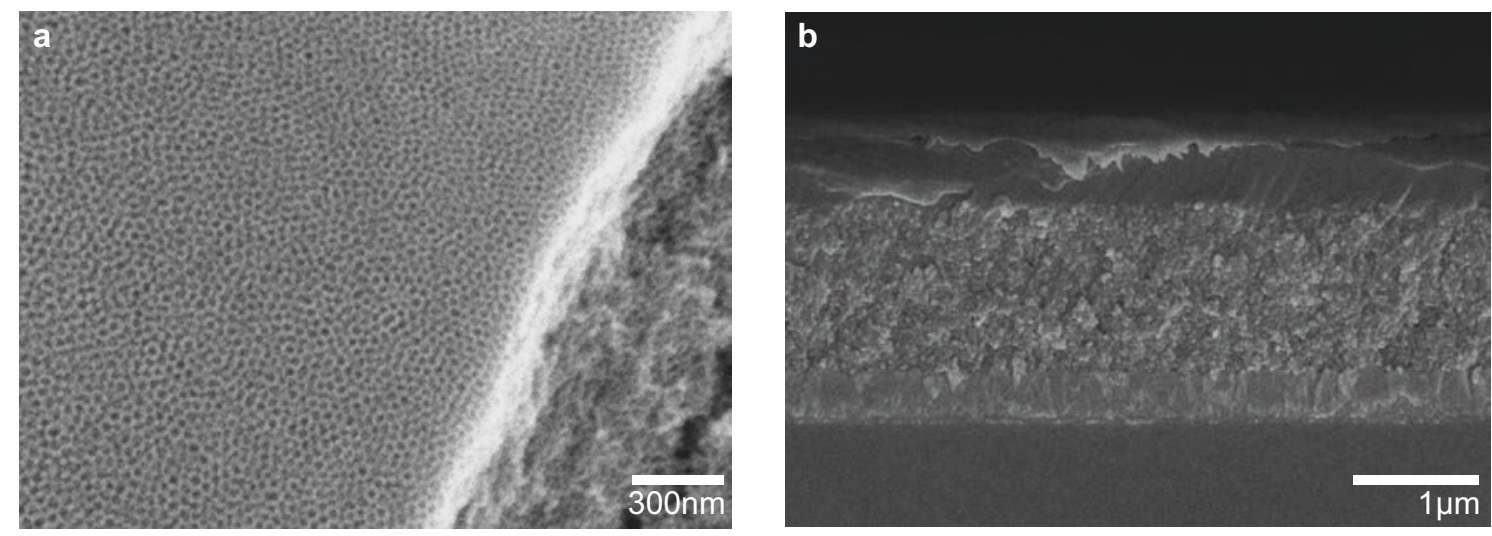

Figure 7.8: Electron micrographs of the photoelectrode morphology. a) SEM top-view of a $1.3 \mu \mathrm{m}$ thick mesoporous $\mathrm{TiO}_{2}$ film, assembled in four deposition and annealing cycles. The pores arising from BCP assembly are fully accessible and no overlayer has formed. b) Cross-sectional SEM image of a fully assembled ss-DSC, where the network has been infiltrated with the hole conducting organic material Spiro-OMeTAD. 
photoanode thickness of $2.5 \mu \mathrm{m}$ resulted in a significantly lower $J_{\mathrm{sc}}$, whereas $V_{\mathrm{oc}} \sim 0.87 \mathrm{~V}$ and $F F \sim 0.7$ were retained for this thickness.

$\mathrm{BCP}$-assembled photoanodes have been previously reported to exhibit faster electron transport compared to random NP networks [15]. Figure 7.10 shows a comparison between multilayer BCP-derived photoanodes and devices incorporating standard NP films of the same thickness in terms of charge recombination, transport and collection rates as a function of the applied bias. It is evident that charge transport in a five-layer BCP device is notably faster, indicating excellent electronic connectivity between the segments. For the direct comparison of $\sim 1.7 \mu \mathrm{m}$ thick films, BCP- and NP-derived devices exhibited transport rates of $4500 \mathrm{~s}^{-1}$ and $2250 \mathrm{~s}^{-1}$, respectively, when operated at the maximum power point.

The high transport rates $k_{\text {trans }}$ can be attributed to the fact that the presented route allows the formation of exceptionally large $\mathrm{TiO}_{2}$ nanocrystals within the mesoporous network while
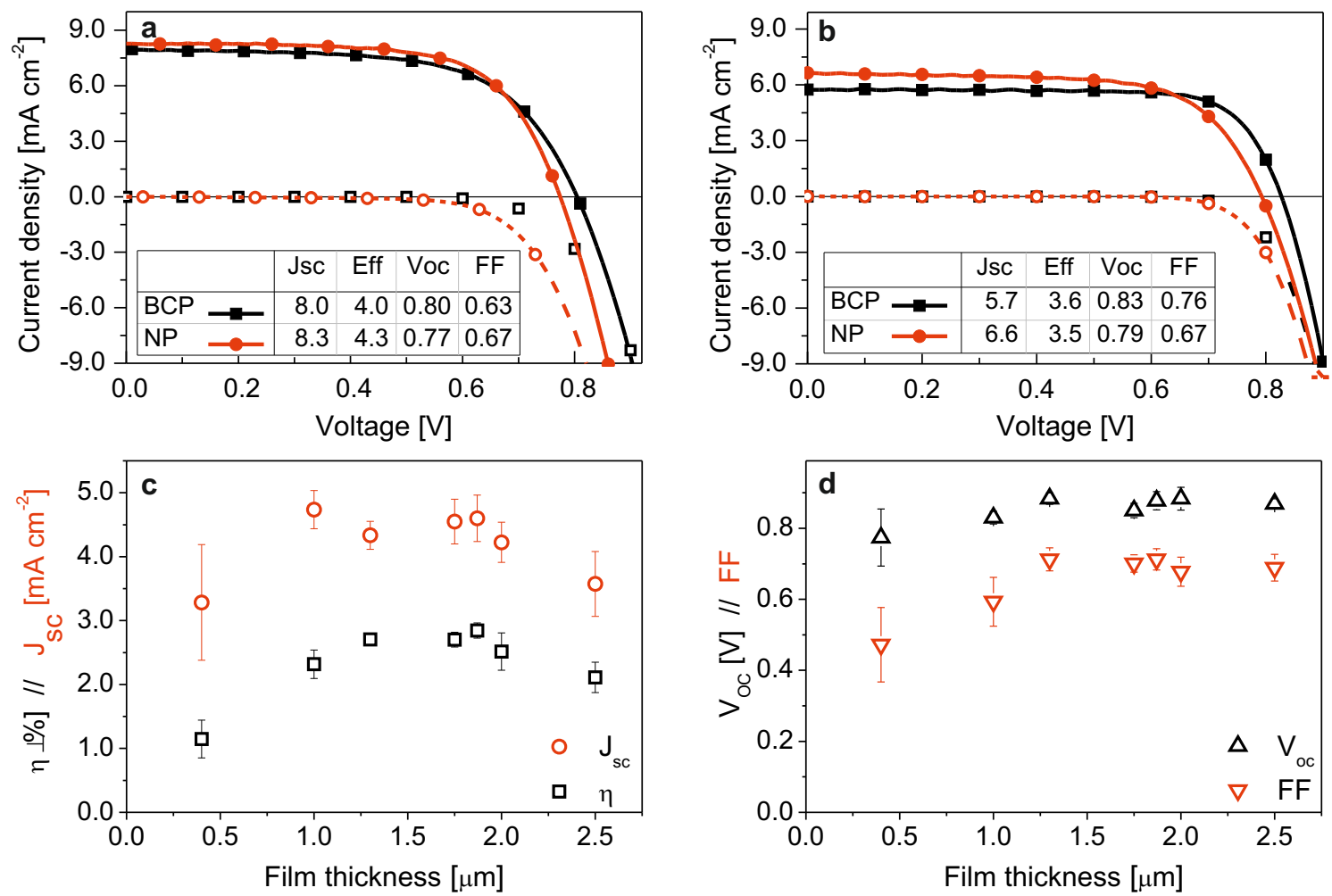

Figure 7.9: Photovoltaic device performance. a) Current-voltage characteristics of the highest efficiency ss-DSCs based on a $1.3 \mu \mathrm{m}$ thick BCP-derived photoanode compared to a NP assembly. The dye CN04 was used in both devices [13]. The filled symbols correspond to a simulated AM 1.5 full sun light illumination $\left(100 \mathrm{~mW} / \mathrm{cm}^{2}\right)$, the open symbols represent the dark current. b) Current-voltage curves of similar devices using the dye D102. c) - d) Key photovoltaic device parameters as a function of the thickness of the active layer. c) Short circuit current $\left(J_{\mathrm{sc}}\right)$ and power conversion efficiency $(\eta)$ of BCP-assembled devices. d) Open-circuit voltage $\left(V_{\mathrm{oc}}\right)$ and fill factor $(F F)$. 

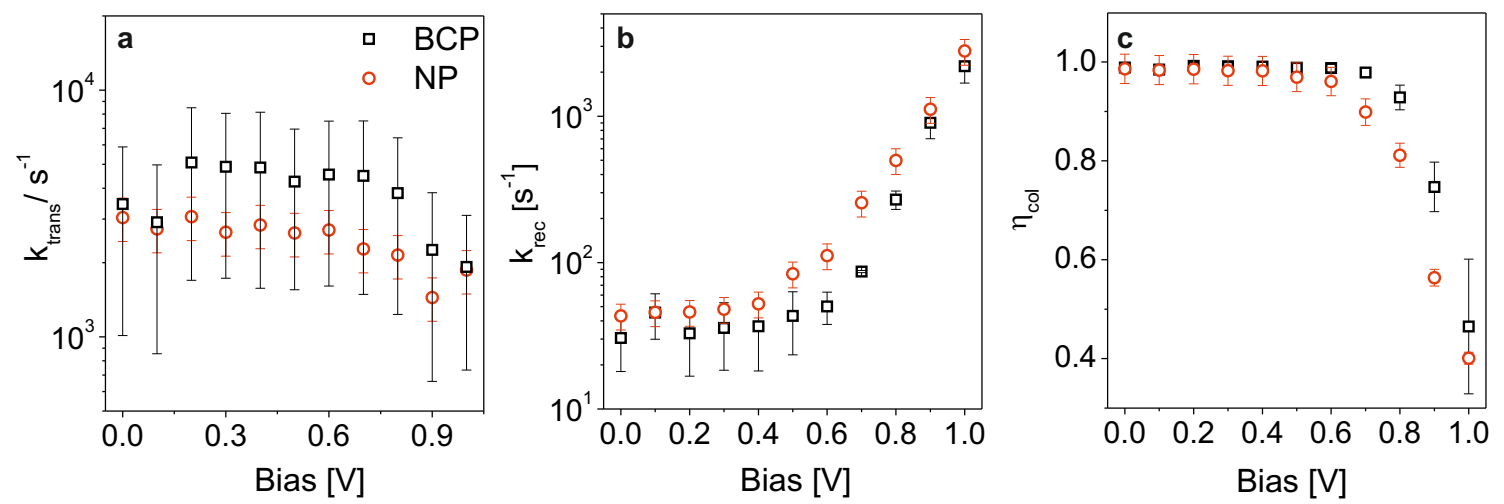

Figure 7.10: Electronic device characteristics. Direct comparison between BCP-assembled photoanodes and conventional NP devices of similar thickness $(1.8 \mu \mathrm{m})$. a) Transport rates $\left(k_{\text {trans }}\right)$, b) recombination rates $\left(k_{\text {rec }}\right)$ and $\left.\mathbf{c}\right)$ charge carrier collection efficiencies $\left(\eta_{\text {col }}\right)$ as a function of the applied bias. While the collection efficiency for standard NP devices deteriorates above $0.5 \mathrm{~V}$, BCP-derived devices retain high efficiencies close to $\mathrm{V}_{\mathrm{oc}}$.

maintaining the BCP imposed morphology [15] (see Chapter 6). Charge recombination rates were significantly reduced in the BCP system with $85 \mathrm{~s}^{-1}$, compared to $255 \mathrm{~s}^{-1}$ for the corresponding NP devices at the maximum power point. These highly conducting networks exhibit a somewhat reduced surface area compared to the standard NP-based photoelectrodes [15]. As the recombination rate $k_{\text {rec }}$ is usually linked to the surface-to-volume ratio of the structure, it is expected to see enhanced transport rates and lower recombination [30, 31]. The biasdependent charge collection is therefore remarkably different in both systems. For the NP devices, charge collection efficiency $\left(\eta_{\text {col }}\right)$ decreased above $0.5 \mathrm{~V}$, while charge collection in BCP devices remained close to unity up to $0.7 \mathrm{~V}$. At $V_{\mathrm{oc}}=0.9 \mathrm{~V}$, the charge collection efficiency for the BCP-derived devices $\eta_{\mathrm{col}}=0.86$ compared to $\eta_{\mathrm{col}}=0.56$ for the NP system. This is consistent with the exceptionally high fill factor of 0.76 obtained with some of these devices. These results are particularly important since they promise more efficient operation at higher bias, i.e. an enhanced fill factor. In fact, large fill factors ( $>0.7)$ were observed with the dye D102 for film thicknesses up to $2.5 \mu \mathrm{m}$. Furthermore, the open-circuit potential of the BCP-derived devices was above $0.85 \mathrm{~V}$ for films thicker than $1.0 \mu \mathrm{m}$, which can be explained by the improved transport properties and the narrower distribution of sub-band-gap states [32].

\subsection{Conclusions}

In summary, a straight-forward route is demonstrated for the fabrication of thick mesoporous films with a well defined pore morphology. A study of the time- and temperature-dependent evolution of the shrinkage process of $\sim 700 \mathrm{~nm}$ thick spin-cast layers enabled the identification 
of the optimal processing parameters for layer-by-layer film formation of thick films, avoiding the formation of cracks and delamination by the gradual release of in-plane stresses. Following a fast, simple and effective annealing protocol, film shrinkage is maximised for each deposition cycle, while preserving the polymer to protect the underlying pores. As a consequence, the time and effort to build-up a multilayer stack is drastically reduced. The resulting mesoporous $\mathrm{TiO}_{2}$ photoanodes exhibit a continuous network with large accessible pores. Increased pore size, film thickness and temperature-stability led to greatly enhanced power conversion efficiencies in solid state dye-sensitised solar cells, which are now comparable to the longoptimised NP system. Significantly enhanced charge transport and charge collection rates at high bias make this system a promising candidate to increase the fill factor and open-circuit potential of thick film ss-DSCs.

\section{Bibliography}

[1] S. Guldin, P. Docampo, M. Stefik, G. Kamita, U. Wiesner, H. Snaith, and U. Steiner, "Layer-by-layer formation of block copolymer derived $\mathrm{TiO}_{2}$ for solid state dye-sensitized solar cells," Small, vol. 8, no. 3, pp. 432-440, 2012.

[2] P. Docampo, A. Hey, S. Guldin, R. Gunning, U. Steiner, and H. Snaith, "Pore-filling of spiro-OMeTAD in solid-state DSCs determined via optical reflectometry," Advanced Functional Materials, vol. 22, no. 23, pp. 5010-5019, 2012.

[3] B. O`Regan and M. Grätzel, "A low-cost, high-efficiency solar-cell based on dyesensitized colloidal $\mathrm{TiO}_{2}$ films," Nature, vol. 353, no. 6346, pp. 737-740, 1991.

[4] J. Macak, M. Zlamal, J. Krysa, and P. Schmuki, "Self-organized $\mathrm{TiO}_{2}$ nanotube layers as highly efficient photocatalysts," Small, vol. 3, pp. 300-304, 2007.

[5] T. Brezesinski, J. Wang, J. Polleux, B. Dunn, and S. Tolbert, "Templated nanocrystalbased porous $\mathrm{TiO}_{2}$ films for next-generation electrochemical capacitors.," Journal of The American Chemical Society, vol. 131, no. 5, pp. 1802-9, 2009.

[6] M. Franke, T. Koplin, and U. Simon, "Metal and metal oxide nanoparticles in chemiresistors: does the nanoscale matter?," Small, vol. 2, no. 1, pp. 36-50, 2006.

[7] L. Bonifacio, D. Puzzo, S. Breslav, B. Willey, A. McGeer, and G. Ozin, "Towards the photonic nose: a novel platform for molecule and bacteria identification," Advanced Materials, vol. 22, no. 12, pp. 1351-4, 2010.

[8] T. Brezesinski, D. Fattakhova-Rohlfing, S. Sallard, M. Antonietti, and B. Smarsly, "Highly crystalline $\mathrm{WO}_{3}$ thin films with ordered 3D mesoporosity and improved electrochromic performance," Small, vol. 2, no. 10, pp. 1203-1211, 2006.

[9] S. Burnside, V. Shklover, C. Barbé, P. Comte, F. Arendse, K. Brooks, and M. Grätzel, "Self-organization of $\mathrm{TiO}_{2}$ nanoparticles in thin films," Chemistry Of Materials, vol. 10, no. 9, pp. 2419-2425, 1998.

[10] T. Dittrich, E. Lebedev, and J. Weidmann, "Electron drift mobility in porous $\mathrm{TiO}_{2}$ (anatase)," Physica Status Solidi A, vol. 1998, no. 165, pp. R5-R6, 1998. 
[11] N. Tetreault and M. Grätzel, "Novel nanostructures for next generation dye-sensitized solar cells," Energy E Environmental Science, DOI: 10.1039/C2EE03242B, 2012.

[12] J. Melas-Kyriazi, I.-K. Ding, A. Marchioro, A. Punzi, B. Hardin, G. Burkhard, N. Tetreault, M. Grätzel, J.-E. Moser, and M. McGehee, "The effect of hole transport material pore filling on photovoltaic performance in solid-state dye-sensitized solar cells," Advanced Energy Materials, vol. 1, no. 3, pp. 407-414, 2011.

[13] N. Cai, S.-J. Moon, L. Cevey-Ha, T. Moehl, R. Humphry-Baker, P. Wang, S. Zakeeruddin, and M. Grätzel, "An organic D-pi-A dye for record efficiency solid-state sensitized heterojunction solar cells," Nano Letters, vol. 11, no. 4, pp. 1452-1456, 2011.

[14] E. Crossland, M. Kamperman, M. Nedelcu, C. Ducati, U. Wiesner, D.-M. Smilgies, G. Toombes, M. Hillmyer, S. Ludwigs, U. Steiner, and H. Snaith, "A bicontinuous double gyroid hybrid solar cell," Nano Letters, vol. 9, no. 8, pp. 2807-2812, 2009.

[15] S. Guldin, S. Hüttner, P. Tiwana, M. Orilall, B. Ülgüt, M. Stefik, P. Docampo, M. Kolle, G. Divitini, C. Ducati, S. Redfern, H. Snaith, U. Wiesner, D. Eder, and U. Steiner, "Improved conductivity in dye-sensitised solar cells through block-copolymer confined $\mathrm{TiO}_{2}$ crystallisation," Energy E Environmental Science, vol. 4, no. 1, pp. 225-233, 2011.

[16] S. Choi, M. Mamak, N. Coombs, N. Chopra, and G. Ozin, "Thermally stable twodimensional hexagonal mesoporous nanocrystalline anatase, meso-nc- $\mathrm{TiO}_{2}$ : bulk and crack-free thin film morphologies," Advanced Functional Materials, vol. 14, no. 4, pp. 335-344, 2004.

[17] M. Zukalová, A. Zukal, L. Kavan, M. Nazeeruddin, P. Liska, and M. Grätzel, “Organized mesoporous $\mathrm{TiO}_{2}$ films exhibiting greatly enhanced performance in dye-sensitized solar cells.," Nano Letters, vol. 5, no. 9, pp. 1789-92, 2005.

[18] J. Prochazka, L. Kavan, V. Shklover, M. Zukalová, O. Frank, M. Kalbac, A. Zukal, H. Pelouchova, P. Janda, K. Week, M. Klementova, and D. Carbone, "Multilayer films from templated $\mathrm{TiO}_{2}$ and structural changes during their thermal treatment," Chemistry Of Materials, vol. 20, no. 9, pp. 2985-2993, 2008.

[19] N. Krins, M. Faustini, B. Louis, and D. Grosso, "Thick and crack-free nanocrystalline mesoporous $\mathrm{TiO}_{2}$ films obtained by capillary coating from aqueous solutions," Chemistry Of Materials, vol. 22, no. 23, pp. 6218 - 6220, 2010.

[20] W. Chen, X. Sun, Q. Cai, D. Weng, and H. Li, "Facile synthesis of thick ordered mesoporous $\mathrm{TiO}_{2}$ film for dye-sensitized solar cell use," Electrochemistry Communications, vol. 9, no. 3, pp. 382-385, 2007.

[21] S. Agarwala, M. Kevin, A. Wong, C. Peh, V. Thavasi, and G. Ho, "Mesophase ordering of $\mathrm{TiO}_{2}$ film with high surface area and strong light harvesting for dye-sensitized solar cell," Acs Applied Materials E Interfaces, vol. 2, no. 7, pp. 1844-1850, 2010.

[22] S. Ahn, J. Park, J. Koh, D. Roh, and J. Kim, "Graft copolymer directed synthesis of micron-thick organized mesoporous $\mathrm{TiO}_{2}$ films for solid-state dye-sensitized solar cells," Chemical Communications, vol. 47, no. 20, pp. 5882-5884, 2011.

[23] J. Tao, Y. Sun, M. Ge, X. Chen, and N. Dai, "Non-prefabricated nanocrystal mesoporous $\mathrm{TiO}_{2}$-based photoanodes tuned by a layer-by-layer and rapid thermal process.," ACS Applied Materials $\mathcal{E}$ Interfaces, vol. 2, no. 1, pp. 265-9, 2010. 
[24] M. Wei, Y. Konishi, H. Zhou, M. Yanagida, H. Sugihara, and H. Arakawa, "Highly efficient dye-sensitized solar cells composed of mesoporous titanium dioxide," Journal Of Materials Chemistry, vol. 16, no. 13, pp. 1287-1293, 2006.

[25] M. Nedelcu, S. Guldin, M. Orilall, J. Lee, S. Hüttner, E. Crossland, S. Warren, C. Ducati, P. Laity, D. Eder, U. Wiesner, U. Steiner, and H. Snaith, "Monolithic route to efficient dye-sensitized solar cells employing diblock copolymers for mesoporous $\mathrm{TiO}_{2}$," Journal Of Materials Chemistry, vol. 20, no. 7, pp. 1261-1268, 2010.

[26] S.-H. A. Lee, N. Abrams, P. Hoertz, G. Barber, L. Halaoui, and T. Mallouk, "Coupling of titania inverse opals to nanocrystalline titania layers in dye-sensitized solar cells," Journal Of Physical Chemistry B, vol. 112, no. 46, pp. 14415-14421, 2008.

[27] S. Guldin, M. Kolle, M. Stefik, R. Langford, D. Eder, U. Wiesner, and U. Steiner, "Tunable mesoporous bragg reflectors based on block-copolymer self-assembly," Advanced Materials, vol. 23, no. 32, pp. 3664-3668, 2011.

[28] G. Floudas, R. Ulrich, and U. Wiesner, "Microphase separation in poly(isoprene-bethylene oxide) diblock copolymer melts. I. Phase state and kinetics of the order-to-order transitions," Journal Of Chemical Physics, vol. 110, no. 1, pp. 652-663, 1999.

[29] M. Nedelcu, J. Lee, E. Crossland, S. Warren, M. Orilall, S. Guldin, S. Hüttner, C. Ducati, D. Eder, U. Wiesner, U. Steiner, and H. Snaith, "Block copolymer directed synthesis of mesoporous $\mathrm{TiO}_{2}$ for dye-sensitized solar cells," Soft Matter, vol. 5, no. 1, 2009.

[30] N. Kopidakis, N. Neale, K. Zhu, J. van de Lagemaat, and A. Frank, "Spatial location of transport-limiting traps in $\mathrm{TiO}_{2}$ nanoparticle films in dye-sensitized solar cells," Applied Physics Letters, vol. 87, no. 20, 2005.

[31] K. Zhu, N. Kopidakis, N. Neale, J. van de Lagemaat, and A. Frank, "Influence of surface area on charge transport and recombination in dye-sensitized $\mathrm{TiO}_{2}$ solar cells," Journal Of Physical Chemistry B, vol. 110, no. 50, pp. 25174-25180, 2006.

[32] P. Docampo, S. Guldin, M. Stefik, P. Tiwana, M. Orilall, S. Hüttner, H. Sai, U. Wiesner, U. Steiner, and H. Snaith, "Control of solid-state dye-sensitized solar cell performance by block-copolymer-directed $\mathrm{TiO}_{2}$ synthesis," Advanced Functional Materials, vol. 20, no. 11, pp. $1787-1796,2010$. 


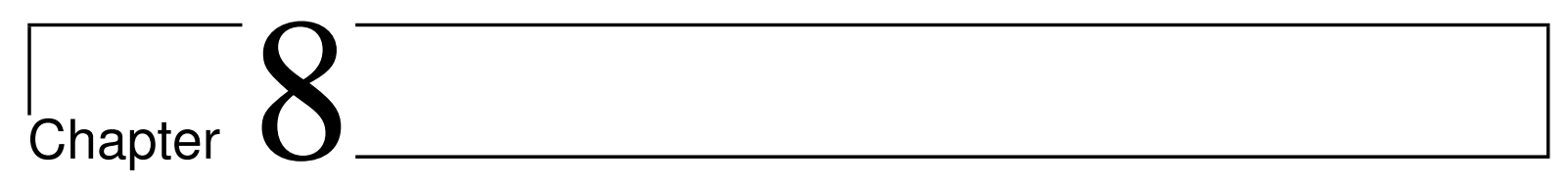

\section{Tunable mesoporous Bragg reflectors based on block copolymer self-assembly}

Published by Stefan Guldin et al. in Advanced Materials (volume 23, page 36643668, 2011) [1] and the Proceedings of the SPIE (DOI: 10.1117/12.893818, 2011) [2].

\subsection{Introduction}

A distributed Bragg reflector (DBR) is defined as a one-dimensional (1D) periodic dielectric lattice of alternating high and low refractive index materials. Reflection of light at each of the layer interfaces leads to multiple interference and thus, a characteristic reflection and transmission behaviour. The underlying theoretical aspects of DBRs are discussed in Chapter 2, including the position of the fundamental Bragg peak $\lambda_{\max }$ (equation 2.32), the width of the reflectance peak $\Delta \lambda_{\max }$ (equation 2.35) and the expected reflectance $\mathcal{R}$ (equation 2.31). Thus, the structural colour response of a multilayer stack can be finely tuned by varying the refractive index, the thickness and the number of alternating layers [3]. DBRs are of technological interest because of their spectrally selective wave guiding properties. Well-established applications include Fabry-Pérot interferometers, optical resonators for distributed feedback lasers and optical cavities for controlling the spontaneous emission rates and spectra from emitting media $[4,5]$. Simple configurations are typically realised by sequential coating of thin solid films of alternating refractive index. Polymer-based DBRs allow mechanical tunability throughout the visible spectrum [6]. Direct access to a 1D dielectric lattice via solution processing has been achieved by the self-assembly of block copolymers (BCPs) with sufficient dielectric contrast into a lamellar morphology [7, 8]. 
An interesting recent area of research is the development of mesoporous DBRs (MDBRs), which access new fields of applications due to their porosity on the sub-optical length scale. Their ability to detect changes in the ambient environment makes them ideally suited as sensing materials. Adsorption and desorption of gas phase molecules in the pores leads to reversible changes in the refractive index of the building blocks and thereby in the photonic properties of the stack $[9,10,11,12]$. MDBRs have been successfully used as biosensors when functionalised to bind specific molecules [13]. This can be extended to an array of MDBRs with different chemical or biochemical functionalisations to allow detection and discrimination of different vapour species such as small molecules and bacteria [14]. The coupling of MDBRs to surface layers [15] and resonance cavities [16] is particularly attractive as it allows sensing with greater accuracy. As outlined in Chapter 3, MDBRs also have great potential in optoelectronic devices. The localisation of light of a specific part of the spectrum leads to efficiency enhancements in dye-sensitised solar cells due to the increase in absorption probability [17]. When infiltrated with light emitting polymers, MDBRs can exhibit distributed feedback lasing [18]. Recently, the coupling of plasmonic particles to MDBR-based resonant cavities has been shown [19].

The material routes towards MDBRs are numerous. Initially, 1D refractive index lattices were realised in porous silicon by periodically changing the etching conditions to achieve high and low porosity layers in an alternating order [20]. In the mid 2000s, Ozin and coworkers introduced the concept of solution-processed MDBRs by the alternate spin coating of Pluronic BCP-assembled mesoporous $\mathrm{TiO}_{2}$ and $\mathrm{SiO}_{2}$ layers [9]. While the morphology within the network showed good structural order, processing was extremely time consuming with fabrication times up to several days for each individual layer of the stack [21]. A less complex route to form porous $\mathrm{TiO}_{2}$ and $\mathrm{SiO}_{2}$ networks is the random packing of nanoparticles (NPs). This was introduced by Cohen et al. [22] and further simplified by Míguez and coworkers. [23]. The optical properties of NP-based MDBRs can be tuned by changing the layer thickness and the materials of the building blocks, but variation of porosity is difficult. The random-close packing of the NPs allows only limited control over the pore fraction and dimensions by changing the NP size and by adding sacrificial material $[24,25]$. The necessary refractive index contrast is therefore typically achieved by stacking $\mathrm{TiO}_{2}$ and $\mathrm{SiO}_{2}$ layers of similar porosity, which leads to electrically insulating MDBRs and therefore limits their application in optoelectronic devices. Furthermore, a random network of sintered NPs lacks detailed control over pore diameters and pore size distribution, limiting functionality for sensing applications and infiltration by other functional materials $[26,27]$. A vacuum-based method that offers fine control over the refractive index is glancing angle deposition (GLAD) that involves physical vapour deposition at an oblique angle $\alpha$ [28]. The ballistic shadowing which arises from tilting the 
substrate with respect to the vapour flux enables to vary the packing density of the nucleated columnar structures [29]. The dynamic variation of $\alpha$ thus allows to fine-tune the refractive index with high precision to form a MDBR [30].

Herein, an alternative concept for the fabrication of MDBRs is presented that relies on the self-assembly of the BCP PI- $b$-PEO in combination with simple hydrolytic sol-gel chemistry. This system enables extended control of porosity and pore size in the resulting inorganic layers by the interplay between the self-assembly of the sacrificial BCP host PI- $b$-PEO and the co-assembled $\mathrm{TiO}_{2}$. Processing conditions are identified that allow for the formation of a multilayer stack with periodic high and low refractive index layers. The effects of this approach on the optical quality and pore accessibility of the resulting MDBRs are theoretically and experimentally characterised.

\subsection{Experimental}

Two stock solutions with differing ratios of $\mathrm{TiO}_{2}$ sol to PI- $b$-PEO BCP were prepared as follows. First, a titanium containing sol was obtained by the rapid addition of $0.69 \mathrm{ml} \mathrm{HCl} \mathrm{(37 \% )}$ to $1 \mathrm{ml}$ titanium ethoxide (purum) under vigorous stirring. In parallel, two polymer solutions were prepared by dissolving $0.1 \mathrm{~g}$ of PI- $b$-PEO (molecular weight $M_{\mathrm{n}}=34.4 \mathrm{~kg} / \mathrm{mol}, 28 \mathrm{wt} \%$ PEO) in $7 \mathrm{ml}$ tetrahydrofuran (THF). After stirring for $90 \mathrm{~min}$, the sol was added to the polymer solution. A weight ratio of 3:1 $\left(\mathrm{TiO}_{2} 3: 1\right)$ and 1:2 $\left(\mathrm{TiO}_{2} 1: 2\right)$ between the resulting $\mathrm{TiO}_{2}$ and the initially used polymer were chosen for the high and low refractive index layers, respectively.

For $\mathrm{TiO}_{2}$ 3:1, $1.31 \mathrm{ml}$ sol was added, followed by the evaporation of volatile components at $50{ }^{\circ} \mathrm{C}$ in a Petri dish. Once dry, the hybrid material was quickly redissolved in a in a solvent mixture of $49 \mathrm{vol} \%$ toluene and $51 \mathrm{vol} \%$ 1-butanol. The solution containing $\mathrm{TiO}_{2}$ 1:2 was prepared similarly, but in this case $0.22 \mathrm{ml}$ sol was added to the polymer base solution and the solvent mixture for redissolving the dried hybrid material consisted of an azeotrope mixture of $72.84 \mathrm{wt} \%$ toluene and $27.16 \mathrm{wt} \%$ 1-butanol. Subsequently the concentrations of the hybrid solutions were adjusted to match the film thickness requirements. Films were then deposited by spin coating $(2000 \mathrm{rpm}, 20 \mathrm{~s})$ onto previously cleaned microscope slides. Subsequent to each film layer deposition, an annealing cycle of $10 \mathrm{~min}$ at $100^{\circ} \mathrm{C}, 200{ }^{\circ} \mathrm{C}$ and $300^{\circ} \mathrm{C}$ was applied. Layers from $\mathrm{TiO}_{2}$ 3:1 and $\mathrm{TiO}_{2}$ 1:2 were deposited in alternating fashion to build-up the MDBR stack. Finally the MDBR was calcined at $500^{\circ} \mathrm{C}$ (three hours, heat ramp $5{ }^{\circ} \mathrm{C} / \mathrm{min}$ ) to remove the organic material and crystallise the $\mathrm{TiO}_{2}$. 


\subsection{Results and discussion}

The BCP PI- $b$-PEO plays a key role in the presented fabrication route as it co-assembles the inorganic material into ordered spatial domains during the phase separation process. The sol acts as a guest that selectively complexes with the PEO phase of the sacrificial BCP host. This provides fine control over the refractive index of the resulting inorganic material through the mixing ratio of the components in the initial solution. In this study, two stock solutions were prepared with mixing ratios of $3: 1\left(\mathrm{TiO}_{2} 3: 1\right)$ and 1:2 $\left(\mathrm{TiO}_{2} 1: 2\right)$ between the $\mathrm{TiO}_{2}$ and the $\mathrm{BCP}$, which then served as a fabrication base for the solution processing of the hybrid films.

A major challenge for the fabrication of BCP-derived mesoporous films is the considerable shrinkage that occurs during the several processing steps. The aim of the presented approach was to establish a processing protocol that could accommodate the shrinkage step-by-step, while minimising the necessary fabrication time. To optimise MDBR fabrication, the film shrinkage process was therefore monitored in situ. $\mathrm{TiO}_{2}$ 3:1 and $\mathrm{TiO}_{2}$ 1:2 were spin-coated onto silicon wafers, and the reflection spectra of individual layers were collected during annealing on a hot plate. Additionally, thermogravimetric analysis and ex situ thickness tracking were employed to understand temperature and time dependence of the shrinkage process (see Chapter 6). Based on these results a $30 \mathrm{~min}$ annealing protocol was established, consisting of three consecutive $10 \mathrm{~min}$ steps at $100^{\circ} \mathrm{C}, 200^{\circ} \mathrm{C}$ and $300{ }^{\circ} \mathrm{C}$. Figure $8.1 \mathrm{a}$ and b shows reflection spectra of $\mathrm{TiO}_{2}$ 3:1 and $\mathrm{TiO}_{2}$ 1:2 hybrid films during the annealing protocol taken in one-minute intervals. The reduction in film thickness shifts the reflectance maxima and minima to smaller wavelengths. For clarity, the spectral position of the maximum reflectance is plotted in Figure 8.1c and $\mathrm{d}$ as a function of time for both mixing ratios. $\mathrm{TiO}_{2}$ 3:1 shrinkage (Figure 8.1b and d) was significantly faster than for the lower inorganic loading of $\mathrm{TiO}_{2} 1: 2$ (Figure 8.1a and c). In both cases, the results indicate that the shrinkage process was completed within the $10 \mathrm{~min}$ interval at each temperature. In this material system a substantial fraction of organic matter is still present in the film after annealing at $300{ }^{\circ} \mathrm{C}$ (see Chapter 7). The sacrificial material plays a crucial role in the stack build-up. The pores of underlying films are thereby protected and cannot clog up during sequential spin-coating, which should lead to highly interconnected inorganic networks after a final calcination step. Cyclic annealing after each layer deposition has another beneficial effect: the condensation of the inorganic network prevents the partial dissolution of the film's surface when contacted by the solution for the next layer. This is essential for the formation of well defined interfaces, a requirement for high optical quality of the MDBRs.

A comparison between the underlying concept and the experimental realisation is shown in Figure 8.2. The dissimilar weight ratios of organic and inorganic material in the two stock 

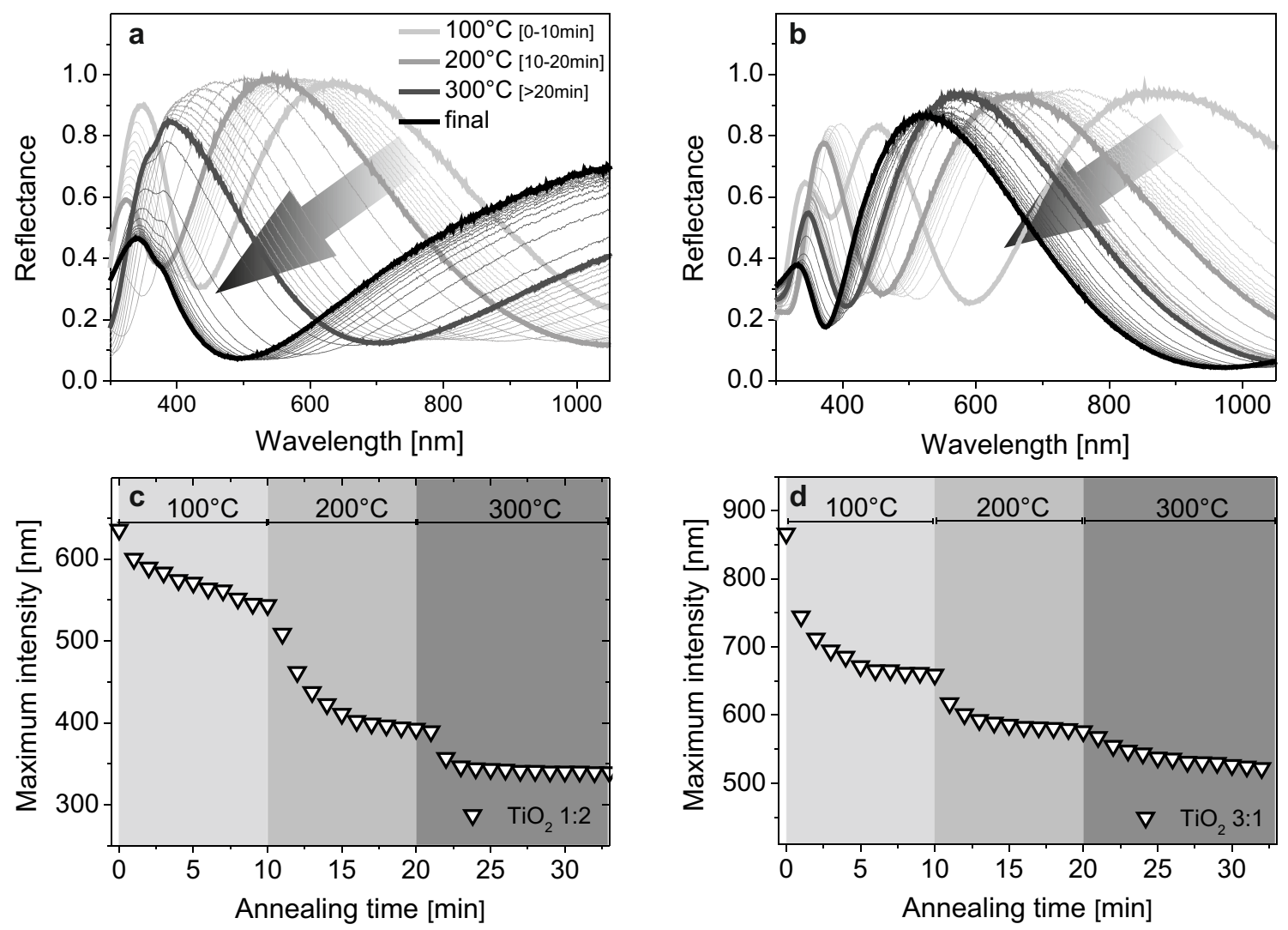

Figure 8.1: Temperature-dependent shrinkage of hybrid films. a) In situ reflectance of a hybrid $\mathrm{TiO}_{2}$ 3:1 film during consecutive annealing at $100^{\circ} \mathrm{C}$ (light grey), $200^{\circ} \mathrm{C}$ (grey) and $300^{\circ} \mathrm{C}$ (dark grey) for $10 \mathrm{~min}$ each. The reflection intensity of the films was normalised by the reflectivity of a bare silicon wafer. b) In situ reflectance of a hybrid $\mathrm{TiO}_{2}$ 1:2 film exposed to the identical temperature protocol. c) - d) Corresponding plots of maximum reflectance intensity for $\mathrm{TiO}_{2} 3: 1$ and $\mathrm{TiO}_{2} 1: 2$, respectively.

solutions results in a different materials arrangement upon phase separation. A higher $\mathrm{TiO}_{2}$ content leads to a denser structure, where less sacrificial pore forming material is incorporated. Increasing the polymer content results in a mainly organic film that yields a more porous network of lower refractive index. A periodic modulation of high and low refractive index in the stack is therefore achieved by spin coating hybrid layers from $\mathrm{TiO}_{2} 3: 1$ and $\mathrm{TiO}_{2}$ 1:2 solutions in alternating fashion, followed by a $30 \mathrm{~min}$ annealing after every film deposition.

Figure 8.2d-f shows the experimental realisation of the MDBR stack structure after calcination at $500{ }^{\circ} \mathrm{C}$ to remove the structure-directing $\mathrm{BCP}$, reveal the pores and crystallise the $\mathrm{TiO}_{2}$ network. Cross-sectional slices were prepared by focussed ion-beam milling to visualise the entire MDBR stack structure. The two different MDBR layers are clearly discernible in the transmission electron microscopy (TEM) image of Figure 8.2d. The darker layers correspond to a high electron density and therefore a high $\mathrm{TiO}_{2}$ content $\left(\mathrm{TiO}_{2} 3: 1\right)$, with the brighter layers stemming from $\mathrm{TiO}_{2}$ 1:2. The films were sputter-coated with platinum for the ion milling 
process. As a consequence, the top layer in this image was partially infiltrated by the metal that is perceptible as black penetration of the top layer.

Top-view scanning electron microscopy (SEM) images of $\mathrm{TiO}_{2} 3: 1$ and $\mathrm{TiO}_{2}$ 1:2 films are presented in Figure 8.2e and f. In contrast to earlier work [31], no solid $\mathrm{TiO}_{2}$ overlayer was observed in any of the thin films. These results imply that in the given wt $\%$ range, the organic structure-directing agent is able to incorporate the full amount of inorganic material into the PEO block, allowing reliable phase separation and refractive index tuning without segregation of inorganic material. The difference in porosity for films stemming from high and low $\mathrm{TiO}_{2}$ loading is clearly visible. $\mathrm{TiO}_{2}$ 1:2 exhibits a very open pore structure with thin walls that are surrounded by a large volume fraction of pores. In contrast, $\mathrm{TiO}_{2} 3: 1$ features a more dense structure with similar pore sizes but a much lower pore density. The morphological dissimilarity results in a significant difference in the effective refractive index of the building blocks, with $n_{1: 2} \sim 1.41$ and $n_{3: 1} \sim 1.69$ as determined by imaging ellipsometry at $\lambda=632 \mathrm{~nm}$. Extending the mixing ratio to higher $\mathrm{TiO}_{2}$ loadings, the refractive index could be tuned to any value between 1.4 and 1.9. This range is well above previously reported solution-based approaches to tune the refractive index by porosity for MDBR manufacture [21, 24, 25].
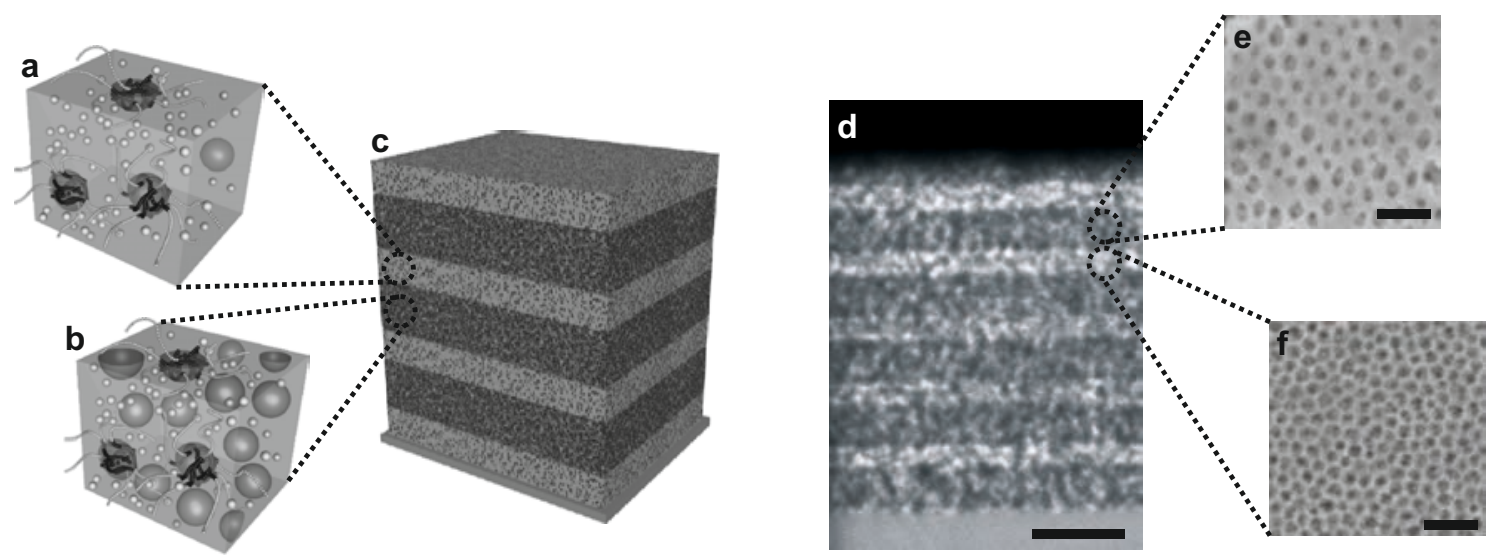

Figure 8.2: Schematic vs. electron micrographs of MDBR structure. a) - c) Schematic of MDBR building blocks. The two stock solutions differ in the $\mathrm{TiO}_{2}$ to polymer weight ratio (3:1 vs. 1:2). The dissimilar ratio of organic and inorganic material is reflected in the micro-phase separation of the co-assembled material after spin coating. a) A higher $\mathrm{TiO}_{2}$ content results in a denser structure with less sacrificial pore forming material. b) Increasing the organic content leads to a more porous network of lower refractive index. b) MDBR stacks are built-up by spin-coating from alternating solutions, followed each time by an annealing step. d) - f) Electron microscopy of the building blocks after calcination at $500{ }^{\circ} \mathrm{C}$. d) TEM cross-sectional view of a MDBR stack. The darker layers reflect the high $\mathrm{TiO}_{2}$ content of $\mathrm{TiO}_{2} 3: 1$, whereas the brighter layers correspond to $\mathrm{TiO}_{2}$ 1:2. e) SEM top view of a $\mathrm{TiO}_{2} 3: 1$ film with a refractive index of $n_{3: 1} \sim 1$.69. e) SEM top view of a highly porous film $\mathrm{TiO}_{2} 1: 2$ film with $n_{1: 2} \sim 1.41$. Scale bars represent $200 \mathrm{~nm}$. 

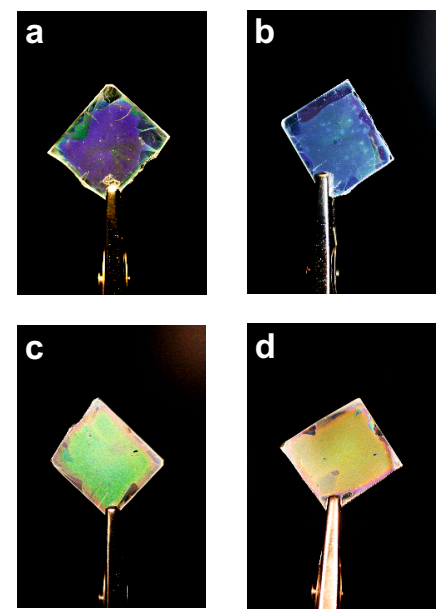

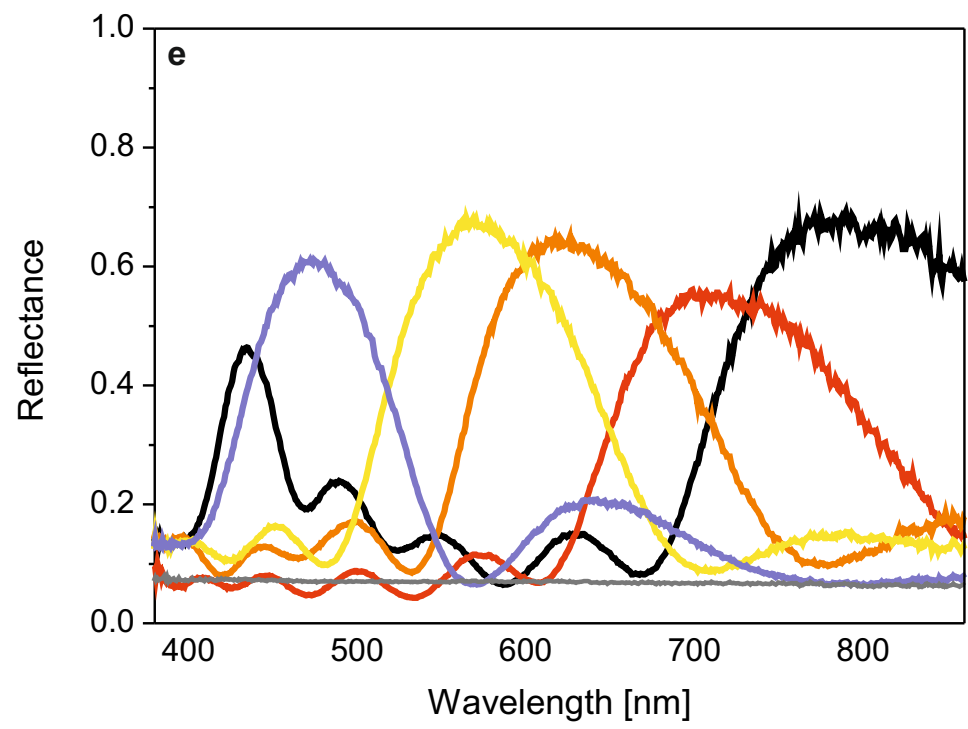

Figure 8.3: Optical properties of MDBRs. a) - d) Photographic images of exemplary MDBRs consisting of five layers $\mathrm{TiO}_{2}$ 3:1 and four layers $\mathrm{TiO}_{2}$ 1:2. The parameters of the $\mathrm{TiO}_{2}$ 3:1 layers were kept constant while the $\mathrm{TiO}_{2}$ 1:2 layers were varied in thickness to give rise to characteristic reflection colours of a) violet, b) blue, c) green and d) yellow. e) Reflectance of MDBRs, where the five layers $\mathrm{TiO}_{2}$ 3:1 were kept constant, while the four layers $\mathrm{TiO}_{2}$ 1:2 were varied in thickness between $78 \mathrm{~nm}$ and $292 \mathrm{~nm}$ to tune the characteristic reflectance across the visible spectrum.

The MDBRs have a well-defined band gap that gives rise to a characteristic colour appearance. This is visible in photographic images of MDBRs shown in Figure 8.3a-d. When altering the thickness of the low refractive index component, the reflected MDBR colour shifts from purple to blue to green to yellow. These images illustrate that the presented method is able to uniformly stack nine layers and maintain a highly uniform optical response. The experimental reflection spectra in Figure 8.3e are in line with the photographs. Here, the reflectance of a nine-layer MDBR was tuned from $\sim 475 \mathrm{~nm}$ to $\sim 800 \mathrm{~nm}$, i.e. across the visible spectrum by varying the concentration of the $\mathrm{TiO}_{2} 1: 2$ solution. While the five $\mathrm{TiO}_{2}$ 3:1 layers were kept constant at $75 \mathrm{~nm}$, the thickness of the $\mathrm{TiO}_{2} 1: 2$ layers was varied between $78 \mathrm{~nm}$ and $202 \mathrm{~nm}$ by adjusting the solution concentration.

In order to evaluate the regularity of the multilayer stack, the experimental results were compared to theoretical calculations of a perfectly ordered dielectric lattice. For this purpose, film parameters of the individual layers were determined by imaging ellipsometry, yielding $n_{3: 1} \sim 1.70 \pm 0.02, h_{3: 1} \sim 76 \pm 2 \mathrm{~nm}, n_{1: 2} \sim 1.40 \pm 0.02$ and $h_{1: 2} \sim 132 \pm 2 \mathrm{~nm}(\lambda=632 \mathrm{~nm})$. Based on these parameters modelling was carried out for a different number of layers by using a home-built Matlab algorithm that follows Rouard's technique [3, 32] (see Chapter 2.3). The modelled optical response of an ideal lattice is compared to experimental reflectance results in Figure $8.4 \mathrm{~b}$, c and $\mathrm{d}$ for five, nine and thirteen layers, respectively. Experimental and theoret- 
ical data bear close resemblance, demonstrating the high optical quality of the samples. This can be attributed to the well defined optical interfaces, the highly reproducible thickness and the refractive index of the layers that are facilitated by the herein presented fabrication route.

Pore interconnectivity is an important property for many technological applications of MDBRs. To confirm the accessibility of the porous network throughout the entire stack, the MDBR was infiltrated by different liquids with refractive indices ranging from 1.33 to 1.55. Figure 8.5a shows the reflection spectra of a nine-layer MDBR in air and after infiltration of the network with $\mathrm{H}_{2} \mathrm{O}$, 2-propanol, chloroform and 1,2-dichlorobenzene. The shift of the Bragg peak to longer wavelengths is the result of a change in the effective refractive index contrast of the layers, as the air in the pores is replaced by the higher refractive index liquids. The dip in maximum reflectance arises from the change of the optical medium at the MDBR's surface (see equation 2.31). The uniform shift of the characteristic Bragg peak suggests a homogeneous infiltration of the optical components from top to bottom. Shown in Figure 8.5b, these results are in good agreement with the modelled shift of the peak position following the symmetric Bruggeman effective medium approximation (see Chapter 2.5). An accessible pore volume of $28 \%$ and $56 \%$ was assumed for $\mathrm{TiO}_{2} 3: 1$ and $\mathrm{TiO}_{2} 1: 2$, respectively.

The herein presented materials route offers several advantages over the state-of-the-art. In contrast to NP-based approaches, the porosity can be finely tuned over a wide parameter space. The pore size of the network is relatively uniform and can be controlled, as it is determined by the molecular weight of the polymer's PI block. It has already been shown that the average pore diameter can be varied in a range from $15 \mathrm{~nm}$ to $55 \mathrm{~nm}$ by using different BCPs [33] (see Chapter 5). This is in contrast to NP networks, where the pore formation is based on the random dense packing of NPs. As a consequence, these random networks suffer from
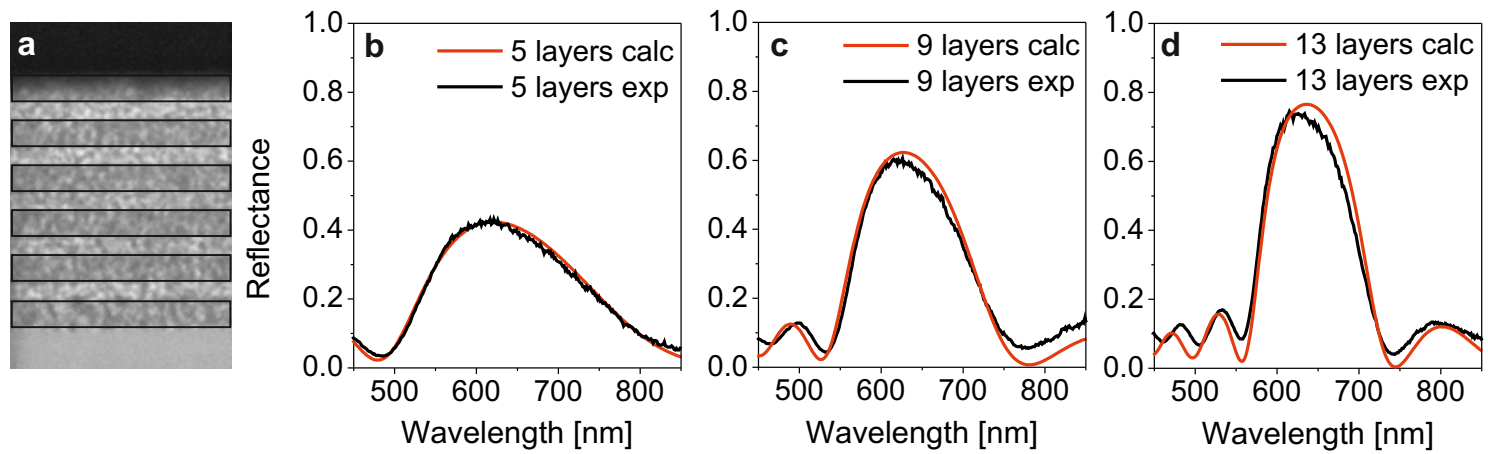

Figure 8.4: Experiment vs. modelling of a perfectly regular stack. a) Segmentation of crosssectional TEM image into layer units. b) - d) Comparison of experimental reflectance with calculations of an perfectly regular stack for b) five, c) nine and d) thirteen layers, respectively. The optical properties of the individual building blocks were determined by ellipsometry. Modelling was carried out using a Matlab algorithm based on Rouard's technique. 

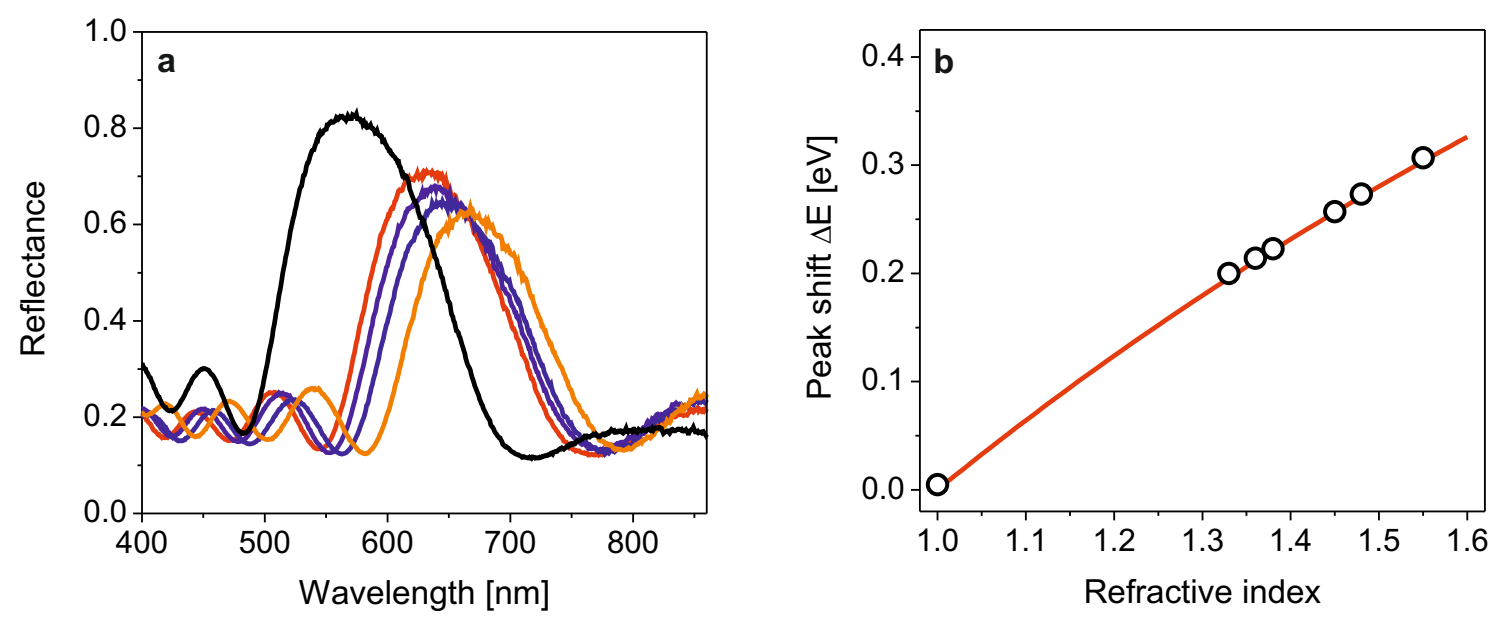

Figure 8.5: Infiltration of an MDBR stack. a) Optical response of a nine-layer MDBR stack in air (black) and after infiltration with $\mathrm{H}_{2} \mathrm{O}$ (red), 2-propanol (blue), chloroform (green) and 1,2dichlorobenzene (orange). b) Corresponding shift of the Bragg peak position as a function of the refractive index for six different infiltrated liquids. The red line corresponds to the theoretical shift of the Bragg peak, calculated by the symmetric Bruggeman effective medium approximation.

a polydisperse pore size distribution and allow only little control over porosity and pore sizes [26]. This route therefore decouples two important parameters in MDBR stack design - porosity and pore size - and allows control of both properties in a wide parameters space. Previous approaches to polymer-directed MDBR fabrication were limited by the extremely long fabrication times and did not allow for significant variation in porosity and pore size $[9,21]$. The present route is fundamentally different due to the properties of the structure-directing agent PI- $b$-PEO. The high Flory-Huggins interaction parameter of the isoprene and ethylene oxide blocks, their low glass-transition temperatures and the high molecular weight are the main reasons why microphase separation occurs orders of magnitude faster than in the commercially available Pluronic (PEO- $b$-PPO- $b$-PEO) [34]. The large PI volume fraction (76.9\%) is the origin of the high porosity, yielding an inorganic material that is less susceptible to shrinkage and structural collapse. On the other hand it allows a large variation in the mixing ratio between the organic host and the inorganic guest, enabling fine control over the refractive index in the mesoporous films in the range of $1.4<n<1.9$. Moreover, the structural stability and fidelity during calcination can be maintained up to $700^{\circ} \mathrm{C}$, which allows superior electronic properties (see Chapter 7).

The presented approach is promising for a wide range of applications. In sensing, adsorptiondesorption properties and their correlated MDBR optical response depend on the pore size as well as the chemical and structural nature of the pore walls [10], all of which can be individually controlled by the presented MDBR manufacture route. The variation of porosity should allow the optical detection of gas phase molecules and surface-binding molecules from solu- 
tion. The fact that the MDBR can now be fully made out of a single, n-type material with full pore interconnectivity is promising for integration into optoelectronic devices, such as hybrid lasers or dye-sensitised solar cells. BCP-derived $\mathrm{TiO}_{2}$ networks have recently shown outstanding performance as electrode materials in solid state dye-sensitised solar cells, which was attributed to the improved conductivity compared to NP films, as well as the manipulation of sub-bandgap states [35, 36]. The MDBR stack of Figure 8.2d is therefore an interesting candidate for the manufacture of dye-sensitised solar cells with integrated DBRs.

\subsection{Conclusions}

To summarise this chapter, a new materials route for MDBR manufacture is presented that harnesses the self-assembly of the PI- $b$-PEO block-copolymer in combination with simple hydrolytic sol-gel chemistry to generate optically uniform layers of well defined porosity. Varying the inorganic-to-organic mixing ratio enabled fine-control of the pore density (and thereby the refractive index), while the pore size can be tuned by the polymer's molecular weight. A 30 min annealing protocol per layer ensures sharp optical interfaces and supports the buildup of a crack-free stack. The resulting MDBRs exhibit a continuous network of pores with well defined dimensions, allowing full accessibility of the film with other functional materials. There is now an abundant range of sol-gel chemistry based approaches that are compatible with block-copolymer assembly $[37,38]$. It is therefore possible to extend the presented $\mathrm{TiO}_{2}$ based MDBRs to a wide range of ceramics and metal oxides. With the fine control over pore size, porosity and material composition, this approach presents new opportunities for MDBR manufacture and integration into functional devices.

\section{Bibliography}

[1] S. Guldin, M. Kolle, M. Stefik, R. Langford, D. Eder, U. Wiesner, and U. Steiner, "Tunable mesoporous bragg reflectors based on block-copolymer self-assembly," Advanced Materials, vol. 23, no. 32, pp. 3664-3668, 2011.

[2] S. Guldin, M. Kolle, M. Stefik, U. Wiesner, and U. Steiner, "Mesoporous Bragg reflectors - block-copolymer self-assembly leads to building blocks with well defined continuous pores and high control over optical properties," Proceedings of the SPIE, vol. 8095, DOI: 10.1117/12.893818, 2011.

[3] O. Heavens, The optical properties of thin solid films. Dover, 1992.

[4] P. Yeh, Optical waves in layered media. Wiley-Blackwell, 2nd ed., 2005.

[5] E. Schubert, Light-emitting diodes. Cambridge University Press, 2nd ed., 2006. 
[6] M. Kolle, B. Zheng, N. Gibbons, J. Baumberg, and U. Steiner, "Stretch-tuneable dielectric mirrors and optical microcavities," Optics Express, vol. 18, no. 5, pp. 575-581, 2010.

[7] A. Urbas, Y. Fink, and E. Thomas, "One-dimensionally periodic dielectric reflectors from self-assembled block copolymer-homopolymer blends," Macromolecules, vol. 32, no. 14, pp. 4748-4750, 1999.

[8] J. Yoon, W. Lee, and E. Thomas, "Self-assembly of block copolymers for photonicbandgap materials," MRS Bulletin, vol. 30, no. 10, pp. 721-726, 2005.

[9] S. Choi, M. Mamak, G. von Freymann, N. Chopra, and G. Ozin, "Mesoporous Bragg stack color tunable sensors," Nano Letters, vol. 6, no. 11, pp. 2456-2461, 2006.

[10] C. Fuertes, S. Colodrero, G. Lozano, A. González-Elipe, D. Grosso, C. Boissière, C. Sánchez, G. J. d. A. Soler-Illia, and H. Míguez, "Sorption properties of mesoporous multilayer thin films," Journal of Physical Chemistry C, vol. 106, pp. 3157-3163, 2008.

[11] L. Bonifacio, B. Lotsch, D. Puzzo, F. Scotognella, and G. Ozin, "Stacking the nanochemistry deck: structural and compositional diversity in one-dimensional photonic crystals," Advanced Materials, vol. 21, no. 16, pp. 1641-1646, 2009.

[12] J. Kobler, B. Lotsch, G. Ozin, and T. Bein, "Vapor-sensitive bragg mirrors and optical isotherms from mesoporous nanoparticle suspensions," ACS Nano, vol. 3, no. 7, pp. 1669-1676, 2009.

[13] V. Lin, K. Motesharei, K. Dancil, M. Sailor, and M. Ghadiri, "A porous silicon-based optical interferometric biosensor," Science, vol. 278, no. 5339, pp. 840-843, 1997.

[14] L. Bonifacio, D. Puzzo, S. Breslav, B. Willey, A. McGeer, and G. Ozin, "Towards the photonic nose: a novel platform for molecule and bacteria identification," Advanced Materials, vol. 22, no. 12, pp. 1351-4, 2010.

[15] N. Hidalgo, M. Calvo, and H. Míguez, "Mesostructured thin films as responsive optical coatings of photonic crystals.," Small, vol. 5, no. 20, pp. 2309-15, 2009.

[16] O. Sánchez-Sobrado, M. Calvo, N. Núñez, M. Ocaña, G. Lozano, and H. Míguez, "Environmentally responsive nanoparticle-based luminescent optical resonators," Nanoscale, vol. 2, no. 6, p. 936, 2010.

[17] S. Colodrero, A. Mihi, L. Haggman, M. Ocaña, G. Boschloo, A. Hagfeldt, and H. Míguez, "Porous one-dimensional photonic crystals improve the power-conversion efficiency of dye-sensitized solar cells," Advanced Materials, vol. 21, no. 7, p. 764770, 2009.

[18] D. Puzzo, F. Scotognella, M. Zavelani-Rossi, M. Sebastian, A. Lough, I. Manners, G. Lanzani, R. Tubino, and G. Ozin, "Distributed feedback lasing from a composite poly(phenylene vinylene)-nanoparticle one-dimensional photonic crystal.," Nano Letters, vol. 9, no. 12, pp. 4273-8, 2009.

[19] N. Hidalgo, M. Calvo, M. Bellino, G. Soler-Illia, and H. Míguez, "Porous supramolecularly templated optical resonators built in 1D photonic crystals," Advanced Functional Materials, vol. 21, no. 13, pp. 2534-2540, 2011.

[20] G. Vincent, "Optical-properties of porous silicon superlattices," Applied Physics Letters, vol. 64, no. 18, pp. 2367-2369, 1994. 
[21] M. Fuertes, F. Lopez-Alcaraz, M. Marchi, H. Troiani, V. Luca, H. Míguez, and G. SolerIllia, "Photonic crystals from ordered mesoporous thin-film functional building blocks," Advanced Functional Materials, vol. 17, no. 8, pp. 1247-1254, 2007.

[22] Z. Wu, D. Lee, M. Rubner, and R. Cohen, "Structural color in porous, superhydrophilic, and self-cleaning $\mathrm{SiO}_{2} / \mathrm{TiO}_{2}$ Bragg stacks.," Small, vol. 3, no. 8, pp. 1445-51, 2007.

[23] S. Colodrero, M. Ocaña, A. Gonzalez-Elipe, and H. Míguez, "Response of nanoparticlebased one-dimensional photonic crystals to ambient vapor pressure," Langmuir, vol. 24, no. 21, pp. 9135-9139, 2008.

[24] M. Calvo, S. Colodrero, T. Rojas, J. Anta, M. Ocana, and H. Míguez, "Photoconducting Bragg mirrors based on $\mathrm{TiO}_{2}$ nanoparticle multilayers," Advanced Functional Materials, vol. 18 , pp. $2708-2715,2008$.

[25] C. Lopez-Lopez, S. Colodrero, S. Raga, H. Lindstrom, F. Fabregat-Santiago, J. Bisquert, and H. Míguez, "Enhanced diffusion through porous nanoparticle optical multilayers," Journal Of Materials Chemistry, vol. 22, no. 5, pp. 1751-1757, 2012.

[26] S. Burnside, V. Shklover, C. Barbé, P. Comte, F. Arendse, K. Brooks, and M. Grätzel, "Self-organization of $\mathrm{TiO}_{2}$ nanoparticles in thin films," Chemistry Of Materials, vol. 10, no. 9, pp. 2419-2425, 1998.

[27] J. Melas-Kyriazi, I.-K. Ding, A. Marchioro, A. Punzi, B. Hardin, G. Burkhard, N. Tetreault, M. Grätzel, J.-E. Moser, and M. McGehee, "The effect of hole transport material pore filling on photovoltaic performance in solid-state dye-sensitized solar cells," Advanced Energy Materials, vol. 1, no. 3, pp. 407-414, 2011.

[28] M. Hawkeye and M. Brett, "Glancing angle deposition: fabrication, properties, and applications of micro- and nanostructured thin films," Journal Of Vacuum Science E Technology A, vol. 25, no. 5, pp. 1317-1335, 2007.

[29] M. Hawkeye and M. Brett, "Controlling the optical properties of nanostructured $\mathrm{TiO}_{2}$ thin films," Physica Status Solidi A-Applications And Materials Science, vol. 206, no. 5, pp. 940-943, 2009.

[30] M. Hawkeye and M. Brett, "Optimized colorimetric photonic-crystal humidity sensor fabricated using glancing angle deposition," Advanced Functional Materials, vol. 21, no. 19, pp. 3652-3658, 2011.

[31] S. Guldin, S. Hüttner, M. Kolle, M. Welland, P. Müller-Buschbaum, R. Friend, U. Steiner, and N. Tetreault, "Dye-sensitized solar cell based on a three-dimensional photonic crystal," Nano Letters, vol. 10, no. 7, pp. 2303-2309, 2010.

[32] M. Kolle, Photonic structures inspired by nature. PhD thesis, University of Cambridge, Physics Department, 2010.

[33] M. Nedelcu, J. Lee, E. Crossland, S. Warren, M. Orilall, S. Guldin, S. Hüttner, C. Ducati, D. Eder, U. Wiesner, U. Steiner, and H. Snaith, "Block copolymer directed synthesis of mesoporous $\mathrm{TiO}_{2}$ for dye-sensitized solar cells," Soft Matter, vol. 5, no. 1, 2009.

[34] G. Floudas, R. Ulrich, and U. Wiesner, "Microphase separation in poly(isoprene-bethylene oxide) diblock copolymer melts. I. Phase state and kinetics of the order-to-order transitions," Journal Of Chemical Physics, vol. 110, no. 1, pp. 652-663, 1999. 
[35] P. Docampo, S. Guldin, M. Stefik, P. Tiwana, M. Orilall, S. Hüttner, H. Sai, U. Wiesner, U. Steiner, and H. Snaith, "Control of solid-state dye-sensitized solar cell performance by block-copolymer-directed $\mathrm{TiO}_{2}$ synthesis," Advanced Functional Materials, vol. 20, no. 11 , pp. $1787-1796,2010$.

[36] S. Guldin, S. Hüttner, P. Tiwana, M. Orilall, B. Ülgüt, M. Stefik, P. Docampo, M. Kolle, G. Divitini, C. Ducati, S. Redfern, H. Snaith, U. Wiesner, D. Eder, and U. Steiner, "Improved conductivity in dye-sensitised solar cells through block-copolymer confined $\mathrm{TiO}_{2}$ crystallisation," Energy E Environmental Science, vol. 4, no. 1, pp. 225-233, 2011.

[37] P. Yang, D. Zhao, D. Margolese, B. Chmelka, and G. Stucky, "Generalized syntheses of large-pore mesoporous metal oxides with semicrystalline frameworks," Nature, vol. 396, no. 6707, pp. 152-155, 1998.

[38] C. Sanchez, C. Boissière, D. Grosso, C. Laberty, and L. Nicole, "Design, synthesis, and properties of inorganic and hybrid thin films having periodically organized nanoporosity," Chemistry Of Materials, vol. 20, no. 3, pp. 682-737, 2008. 



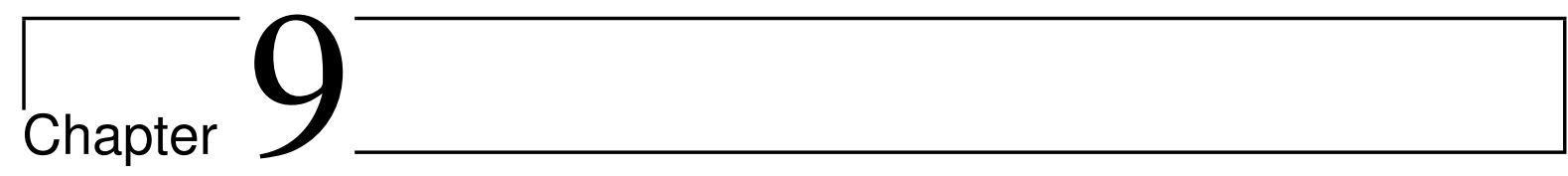

\section{Dye-sensitised solar cell based on a three-dimensional photonic crystal}

Published by Stefan Guldin et al. in Nano Letters (volume 10, page 2303-2309, 2010) [1] and in Proceedings of the SPIE (DOI: 10.1117/12.893798, 2011) [2].

\subsection{Introduction}

Ever since the pioneering work of O'Regan and Grätzel, dye-sensitised solar cells (DSCs) have attracted great interest as a promising technology for future sustainable energy generation [3]. The operation principle of a DSC as well as current challenges in device development are discussed in Chapter 3. In general, improvements in the overall power conversion efficiency have been focussed on further development of new dyes, manipulating the regeneration process by the use of alternative redox couples, improving the electronic properties of the n-type photoanode material and increasing long term stability by better encapsulation [4]. One way to further improve the efficiency of the device is to broaden the region of maximum incident photon-to-electron conversion efficiency (IPCE). The IPCE can reach values around $90 \%$ at wavelengths of high dye extinction, typically in a spectral range from $510-560 \mathrm{~nm}$. In contrast, the IPCE drops significantly in the tail of the absorption spectrum [5]. The engineering of dyes with a panchromatic absorption spectrum is therefore of central importance [6]. A complimentary route to reach broader absorption is the integration of optical elements in the device architecture. These can increase the optical path of the incident light and thereby the probability of the photons to be absorbed. Scattering layers in form of $\mathrm{TiO}_{2}$ particles of several hundred nanometres in size have been found particularly beneficial $[7,8]$ and are now a common element in record efficiency cells $[9,10]$. While the implementation of scattering particles has proven highly beneficial for enhanced light absorption, the diffusive scattering 
over a broad spectral range has the detrimental effect of turning the cells opaque. This deprives the DSC from one of its unique selling points and thus, its potential use for integrated architecture. As a result, photonic band gap materials in the form of three-dimensional (3D) inverted $\mathrm{TiO}_{2}$ opal or porous Bragg stacks have been applied to DSCs in order to enhance light harvesting in specific parts of the spectrum while retaining the cell's transparency in others $[11,12,13,14]$. Several theoretical approaches report a variety of possible effects, including the slow propagation of photons near the edges of a photonic gap [15], Bragg diffraction in a periodic dielectric lattice [16], multiple scattering at disordered regions in the photonic crystal (PC) [17] and the formation of multiple resonant modes [18]. The latter effect, predicted by Mihi and Míguez [18] via a scalar wave approximation, has generated interest as it promises significant enhancement over a large part of the spectrum. According to these calculations, resonant cavities, determined by the penetration depth of the incoming light and the thickness of the mesoporous film between the PC and the substrate, give rise to photon localisation in the cell and therefore a much higher probability of photon absorption (see Chapter 3.2). The main challenge for the integration of a 3D PC into the DSC device structure lies in the appropriate coupling of the layers. An opaline array grown onto the mesoporous layer by evaporation induced self-assembly can only serve as a template, as to realise the necessary refractive index contrast. This has to be converted by infiltration with a high refractive index material, typically $\mathrm{TiO}_{2}$, without clogging the mesoscopic pores of the underlayer that would otherwise inhibit sensitisation and electrolyte infiltration of the double layer device. In early approaches inverse opal PC layers could only be deposited directly on the transparent electrode rather than on top of a mesoporous $\mathrm{TiO}_{2}$ layer $[11,12]$. In order to minimise direct back-reflection of the incident photons, the cells had to be illuminated from the backside of the device, leading to a significant loss due to absorption in the platinum counter-electrode and the excess path length through the electrolyte [19]. Míguez and coworkers proposed that resonant modes, covering a large part of the spectrum, could only be exploited if the mesoporous $\mathrm{TiO}_{2}$ layer was positioned in between the PC and the conductive substrate [20]. This was attempted in several studies [21,22]. Recently, Lee et al. presented a new material route that enabled the beneficial layer sequence by infiltrating and thereby protecting the mesoporous underlayer with a copolymer during the subsequent fabrication of the inverse opal top layer. [23]. The effect of such a double layer configuration was subsequently characterised in great detail. According to their findings the material lacked the necessary intimate physical contact between the mesoporous and inverse opal layers to induce resonant modes. Furthermore, poor coupling of the two layers prevented charge carriers that were generated in the inverse opal layer to contribute to the overall photocurrent. This study emphasised the need for an approach to simultaneously deposit the mesoporous $\mathrm{TiO}_{2}$ and the pore protective material in order to achieve pore and 
electrical contact between the layers and thus fully integrate the PC into the device.

In this chapter a novel material route is presented for the assembly of a double layer DSC that couples an optically active 3D PC overlayer with a high surface mesoporous underlayer. Self-assembly-directed material synthesis on two length-scales enabled the fabrication of a double layer DSC with electric and pore connectivity at the mesoporous and the microporous level. Subsequent device characterisation allowed correlation of the construct's photonic properties to light harvesting and overall performance of the resulting DSC.

\subsection{Experimental}

For the fabrication of the block-copolymer (BCP) assembled mesoporous underlayer, a titanium containing sol was prepared by the rapid addition of $0.69 \mathrm{ml} \mathrm{HCl} \mathrm{(37 \% )} \mathrm{to} 1 \mathrm{ml}$ titanium ethoxide (purum) under vigorous stirring. A solution of $0.1 \mathrm{~g}$ PI- $b$-PEO BCP $\left(M_{\mathrm{n}}=\right.$ $35.7 \mathrm{~kg} / \mathrm{mol}, 23 \mathrm{wt} \%$ PEO) in $7 \mathrm{ml}$ of tetrahyfrofuran (THF) was prepared alongside. After $90 \mathrm{~min}$ of stirring, $0.873 \mathrm{ml}$ of the resulting sol was added to the polymer solution, before the volatile components were evaporated at $50^{\circ} \mathrm{C}$ in a Petri dish. Shortly after, the dry material was redissolved in an azeotrope mixture of of $72.84 \mathrm{wt} \%$ toluene and $27.16 \mathrm{wt} \%$ 1-butanol. Films were then deposited by spin-coating of the $20 \mathrm{wt} \%$ solution $(5 \mathrm{~s}, 1500 \mathrm{rpm})$. After annealing the films by slow ramping up to $130^{\circ} \mathrm{C}$ (180 min linear ramp, $30 \mathrm{~min}$ dwell), a thin overlayer was removed by reactive ion etching $\left(\mathrm{CF}_{4}, 2 \mathrm{~min}, 50 \mathrm{~W}, 16 \mathrm{mbar}, 3.56 \mathrm{MHz}, 310 \mathrm{~V}\right.$, homebuild system). The 3D PC was subsequently fabricated on top of this layer in three steps: a face-centred-cubic (FCC) colloidal array was deposited by evaporation induced self-assembly of polystyrene spheres. Three different sphere diameters $\left(d_{\mathrm{iam}}\right)$ were used for comparison: $240 \mathrm{~nm}$ (PC1, $0.5 \mathrm{vol} \%$ in ethanol, Ikerlat), $260 \mathrm{~nm}$ (PC2, $1.0 \mathrm{vol} \%$ in ethanol, Ikerlat) and $350 \mathrm{~nm}$ (PC3, $0.5 \mathrm{vol} \%$ in ethanol, Polybead). The template was then infiltrated by a binary atmospheric pressure chemical vapour deposition (APCVD). Subsequently, the samples were exposed by another cycle of reactive ion etching $\left(\mathrm{CF}_{4}, 5 \mathrm{~min}\right.$, see conditions above). Finally, the $\mathrm{TiO}_{2}$ double layer structure was revealed by the calcination of the samples at $500{ }^{\circ} \mathrm{C}$. For device characterisation, DSCs were assembled following a protocol as described elsewhere (dye N719) [24]. Please see reference [1] for further experimental details.

\subsection{Results and discussion}

A schematic representation of the fabrication method for a double layer self-assembled DSC is shown in Figure 9.1. In contrast to earlier approaches, a solution of PI- $b$-PEO BCP and $\mathrm{TiO}_{2}$ sol served as fabrication base for the mesoporous underlayer. The layer was deposited 
by spin coating or blade coating and temperature annealed to allow condensation of the inorganic network. At this fabrication stage the microphase-separated hybrid layer exhibits a continuous interdigitation between the $\mathrm{BCP}$ host material and the co-assembled $\mathrm{TiO}_{2}$. As a consequence, the inorganic material is fully surrounded by protective organic material for further processing. The template for the PC top layer was subsequently deposited onto the hybrid film by evaporation induced self-assembly of polystyrene microspheres [25]. The covered substrate was placed vertically in an ethanolic suspension. Upon solvent evaporation, a uniform face-centered-cubic (FCC) colloidal PC template was formed on the hybrid underlayer. This three-dimensional template was then infiltrated by a binary atmospheric pressure chemical vapour deposition (APCVD, [26]). The template was heated to $80^{\circ} \mathrm{C}$ before being subjected to water vapour and titanium tetrachloride vapour in alternating cycles to form amorphous $\mathrm{TiO}_{2}$. Since the hybrid underlayer is non-porous, the APCVD of $\mathrm{TiO}_{2}$ does not clog the

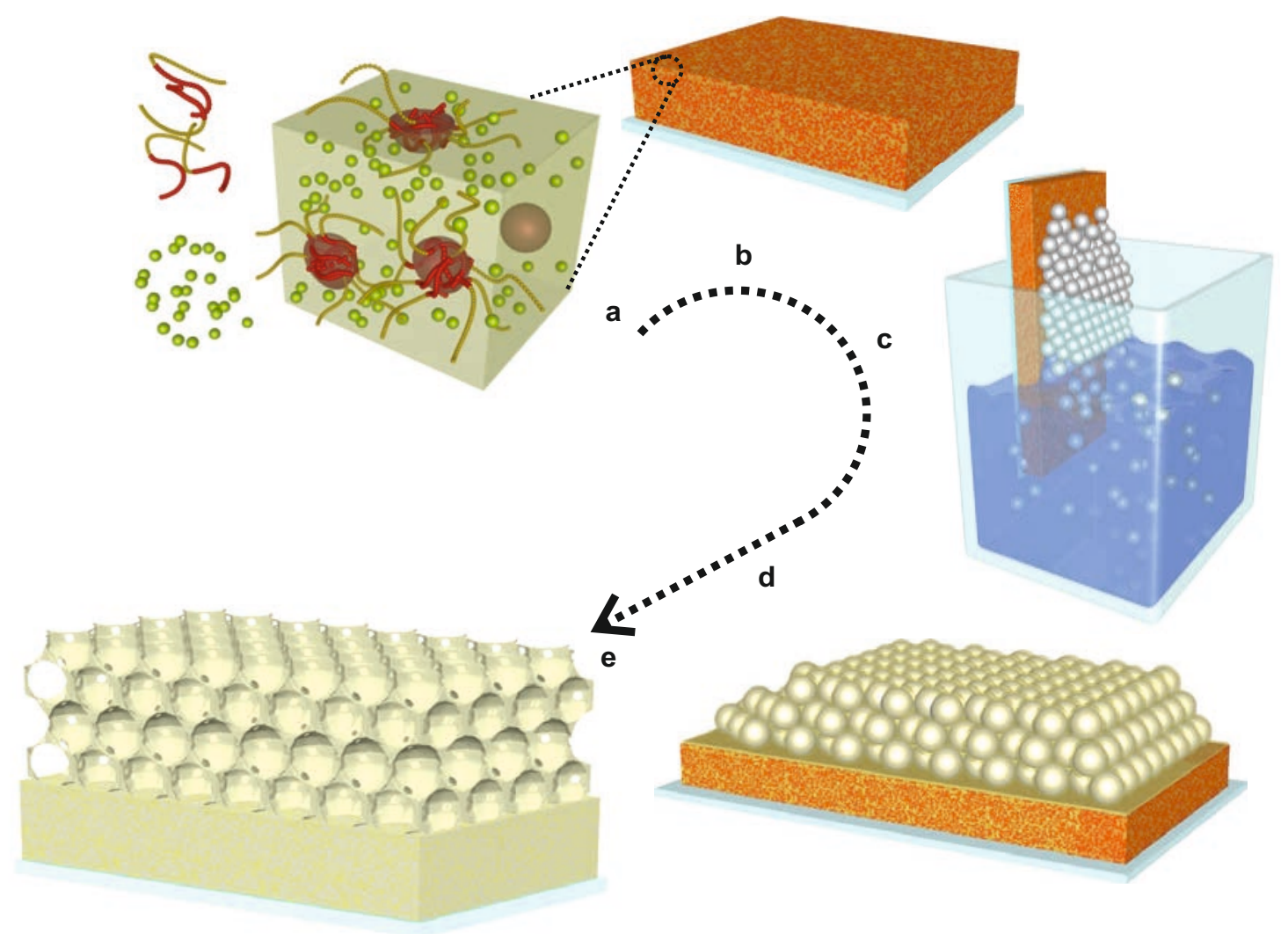

Figure 9.1: Schematic of double layer assembly. a) A solution of PI- $b$-PEO BCP and $\mathrm{TiO}_{2}$ sol is b) co-deposited onto a FTO-covered glass substrate by spin-coating and solvent evaporation. c) The covered substrate is subsequently placed vertically in an ethanolic suspension of polystyrene microspheres to induce the self-assembly of the 3D PC template. d) This is followed by infiltration of the the interstitial pores in the colloidal array by APCVD of amorphous $\mathrm{TiO}_{2}$. e) High temperature calcination removes the $\mathrm{BCP}$ and colloids and crystallises the double layer $\mathrm{TiO}_{2}$ photoanode. 
pores of the underlying $\mathrm{TiO}_{2}$ mesostructure, which would otherwise hinder sensitisation and electrolyte infiltration. After reactive ion etching of the continuous $\mathrm{TiO}_{2}$ top layer, the double layer structure was heated to $500{ }^{\circ} \mathrm{C}$ in order to remove the sacrificial polymer, crystallise the $\mathrm{TiO}_{2}$ and reveal the pores on the $20 \mathrm{~nm}$ and $200 \mathrm{~nm}$ length scale, formed by BCP and colloidal self-assembly in the consecutive layers.

Electron microscopy of the $\mathrm{TiO}_{2}$ double layer morphology is presented in Figure 9.2. The micrographs confirm the successful accomplishment of the fabrication route including the selfassembly of a 3D FCC polystyrene PC template on top of the hybrid thin film, the subsequent APCVD infiltration with $\mathrm{TiO}_{2}$ and final removal of the organic material by calcination. A mesoscopic layer thickness of $\sim 465 \pm 30 \mathrm{~nm}$ was measured by film profilometry, while the PC thickness was about 10 colloidal layers for a 0.5 vol\% suspension and 20 colloidal layers for a 1.0 vol\% suspension, as determined by SEM image analysis. The pore connectivity between both layers is visible in Figure 9.2b, confirming that the fabrication method provided an effective protection for the mesopores during the filling of the PC template by APCVD. This is an important requirement for effective dye sensitisation of the high surface area mesoscopic underlayer as well as the unhindered electrolyte diffusion throughout the photoanode. Furthermore, the APCVD infiltration appeared to be uniform throughout the crystal with no evidence of delamination from the PC overlayer.

Figure 9.3a shows the top surface of the (111) crystal plane of the inverse opal $\mathrm{TiO}_{2} \mathrm{PC}$ layer obtained after deposition of $240 \mathrm{~nm}$ PS spheres, APCVD, $\mathrm{CF}_{4}$ etching of the $\mathrm{TiO}_{2}$ overlayer and high temperature calcination. Figure $9.3 \mathrm{~b}$ displays a photograph of a $\mathrm{TiO}_{2}$ double layer (PC3). Note the complementary colours of the reflected and transmitted light. In Figure
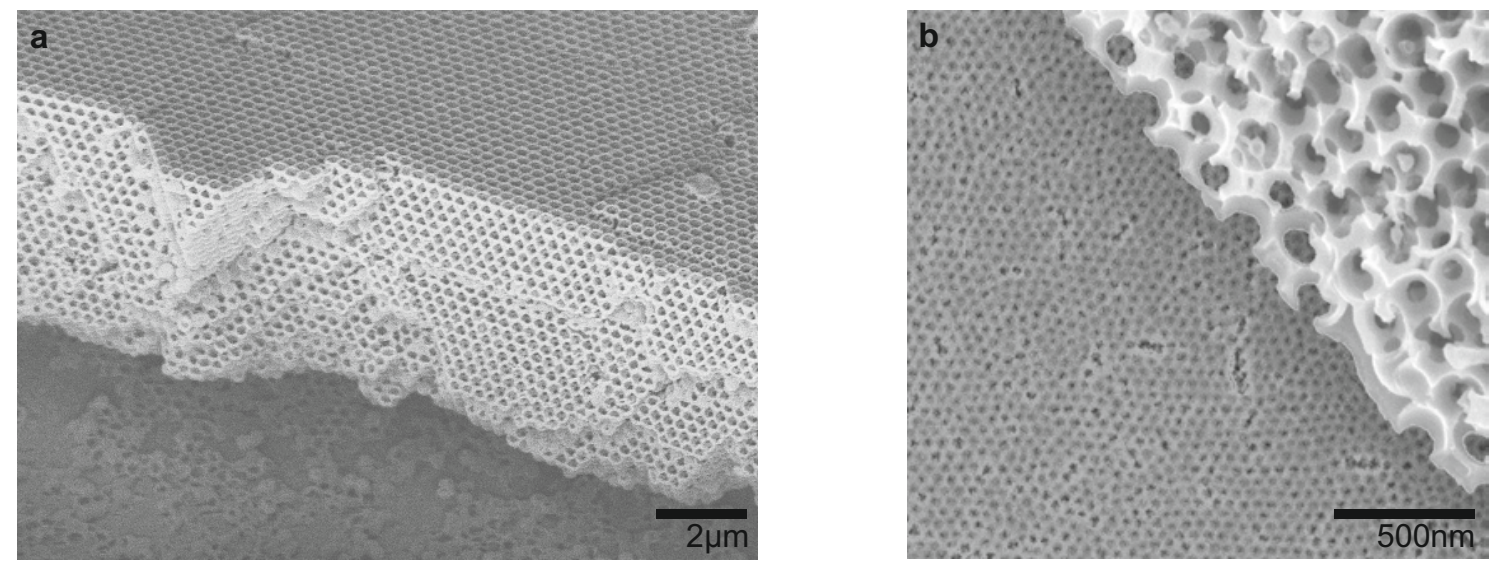

Figure 9.2: Electron microscopy of a $\mathbf{T i O}_{2}$ double layer morphology. SEM cross-sectional micrographs of a) the inversed $\mathrm{TiO}_{2}$ opal $\mathrm{PC}$ on top of a mesoscopic $\mathrm{TiO}_{2}$ underlayer after high temperature calcination. b) High resolution micrograph showing good pore connectivity between mesopores and micropores. a), b) Reprinted from [27]. Copyright Stefan Guldin. 
9.3c the optical transmission spectrum through the self-assembled FCC PC template is shown for all three diameters used in this study. The transmission dips at $500 \mathrm{~nm}$ (PC1), $540 \mathrm{~nm}$ (PC2) and $825 \mathrm{~nm}$ (PC3), corresponding to the Bragg reflection peaks of the (111) $\Gamma$-L pseudo-gap of PS spheres with diameters of $210 \mathrm{~nm}, 227 \mathrm{~nm}$ and $350 \mathrm{~nm}$, respectively $\left(n_{\mathrm{eff}}=1.46\right)$. In Figure 9.3d the transmission spectrum for the different stages of the fabrication process is shown for PC3. After infiltration of the colloidal array with amorphous titania, the transmission dip shifted to $850 \mathrm{~nm}$. Calcination and crystallisation of the $\mathrm{TiO}_{2}$ lattice contraction led to an $\mathrm{TiO}_{2}$ inverse opal with $240 \mathrm{~nm}$ pores, which is in line with the corresponding $\mathrm{TiO}_{2}$ transmission spectrum in air. From these measurements, it follows that the presented fabrication method is capable of producing high quality photonic crystal structures with the expected optical characteristics.
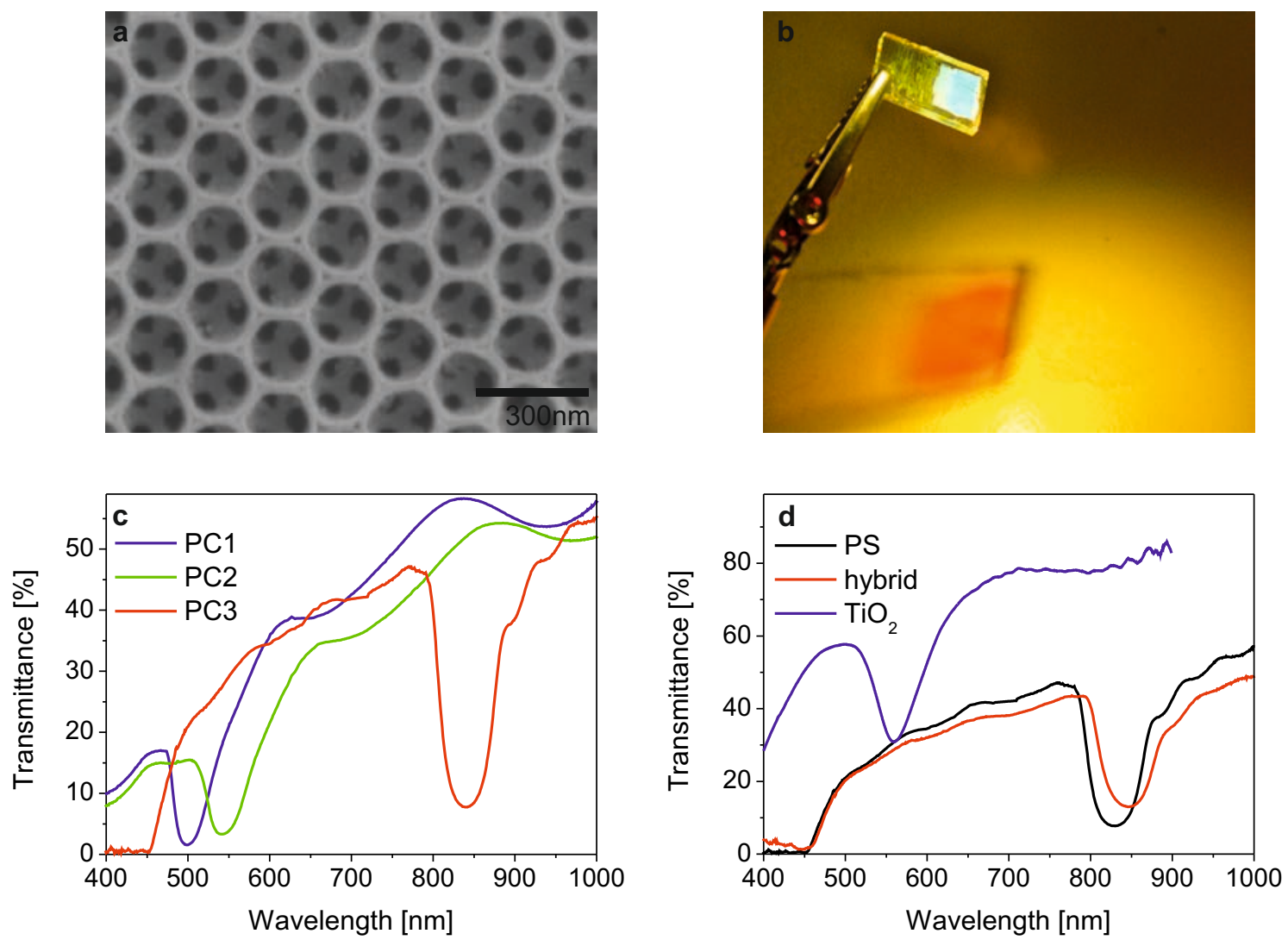

Figure 9.3: Optical properties of 3D PC. a) SEM micrograph showing the (111) top surface of the resulting inverse $\mathrm{TiO}_{2}$ opal $\mathrm{PC} 1$. b) Photograph of a $\mathrm{TiO}_{2}$ double layer with $\mathrm{PC} 3$. c) Uv-vis transmittance spectrum of the colloidal PC templates for PC1 (blue, $d_{\mathrm{iam}}: 240 \mathrm{~nm}$ ), PC2 (green, $d_{\mathrm{iam}}: 260 \mathrm{~nm}$ ) and PC3 (red, $\left.d_{\mathrm{iam}}: 350 \mathrm{~nm}\right)$. d) Transmission spectrum of the PC3 sample for the different stages of the fabrication process: PS colloidal array in air (black), infiltrated template (red) and $\mathrm{TiO}_{2}$ inverse opal in air after calcination (blue). a), c) Reprinted from [27]. Copyright Stefan Guldin. 
In order to reveal the significance of the PC top layer for light harvesting, the IPCE behaviour of double layer DSCs was compared to a single layer reference device. For this study, a relatively thin $\mathrm{TiO}_{2}$ underlayer $(465 \pm 30 \mathrm{~nm})$ was chosen to avoid the formation of cracks. These typically appear in films thicker than about $1 \mu \mathrm{m}$ and may give rise to light scattering. (The findings presented in Chapter 7 would now allow the fabrication of significantly thicker crackfree films.) A double layer device consisting of a $465 \mathrm{~nm}$ underlayer with $32 \%$ porosity and a $1.4 \mu \mathrm{m}$ PC top layer with $78 \%$ porosity was modelled to have a similar surface area as an approximately $900 \mathrm{~nm}$ thick single mesoporous film, which was therefore used as a reference.

The effect of the PC top layer on the absorption spectrum of the overall device was herein of particular interest. Double layer devices incorporating 3D PCs of different pore diameters, related to the sphere size used in their fabrication, were compared to the absorption of a reference cell without a PC but a thicker mesoporous layer. For all IPCE measurements, a $1 \mathrm{~mm}^{2}$ surface was illuminated from the FTO side with an intensity of $100 \mathrm{~mW} / \mathrm{cm}^{2}$ (AM 1.5). The IPCE characteristics of the three PC cell types are shown in Figure 9.4. Two main characteristics of the double layer devices are apparent in the IPCE and normalised IPCE spectra. First, double layer devices with a comparable surface area to that of the single layer reference were found to have comparable peak absorption values in the measured spectral range, as seen for PC1 (a) and PC3 (c). In contrast, PC2 has a significantly thicker PC top layer $(\sim 3 \mu \mathrm{m})$ and therefore, a roughly $40 \%$ higher surface area when compared to the double layer devices PC1 and PC2, or the reference cell. The fact that the IPCE increased accordingly supports the assertion that the PC top layers are electrically connected and contribute to light harvesting over the entire measured spectrum.

The PC layers were also optically characterised. The spectral region of the stop-band in the device is illustrated as hatched areas in Figure 9.4. When normalised to the IPCE maxima in Figure 9.4d-f, the significance of a PC top layer for spectrally selective light harvesting is revealed. Comparing the normalised IPCE of different PC double layer devices with the reference cell, it is evident that the observed absorption spectrum of the device is strongly influenced by the PC top layer. The broadening of the normalised absorption peak moves with the position of the Bragg peak, from deep blue to red. The physical origin of this effect cannot be exclusively related to scattering effects. These would occur most efficiently around $\lambda \sim 1.35 \cdot d_{\text {iam }}$, where $d_{\text {iam }}$ is the diameter of the scattering object, i.e. in the deep blue for all three model systems [28]. Furthermore, scattering at defect planes should be closely related to the ratio of $d_{\text {iam }} / \lambda$, leading to very low scattering probabilities in the observed region of enhancement for all three feature sizes [17]. Nevertheless, a small fraction of the observed absorption shift can be correlated with scattering, best seen in Figure 9.4f as a slightly increased absorption shoulder in the blue. Furthermore, the slight increase in relative absorption between 

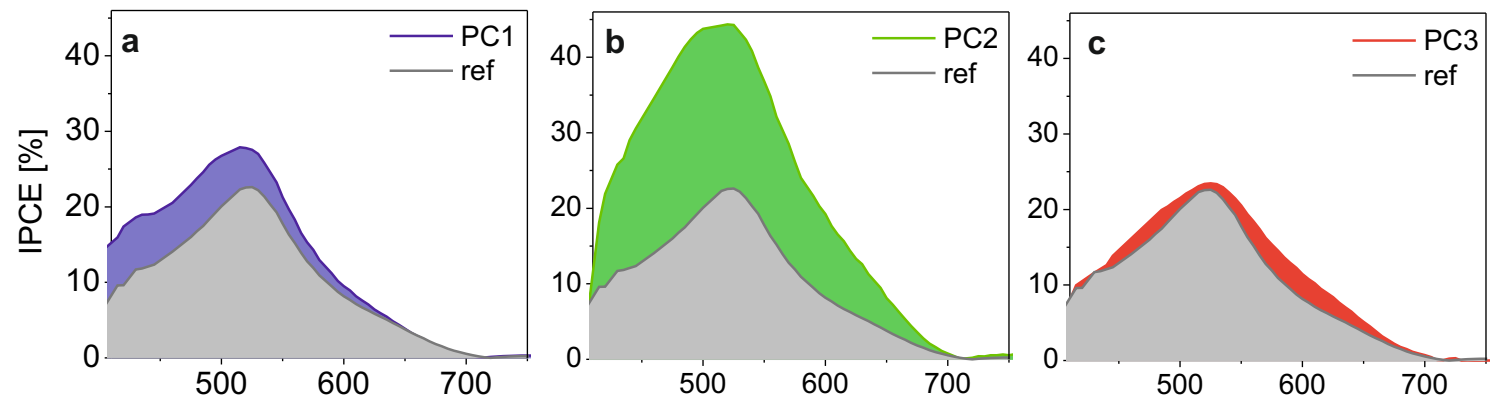

Wavelength $[\mathrm{nm}]$

Wavelength $[\mathrm{nm}]$
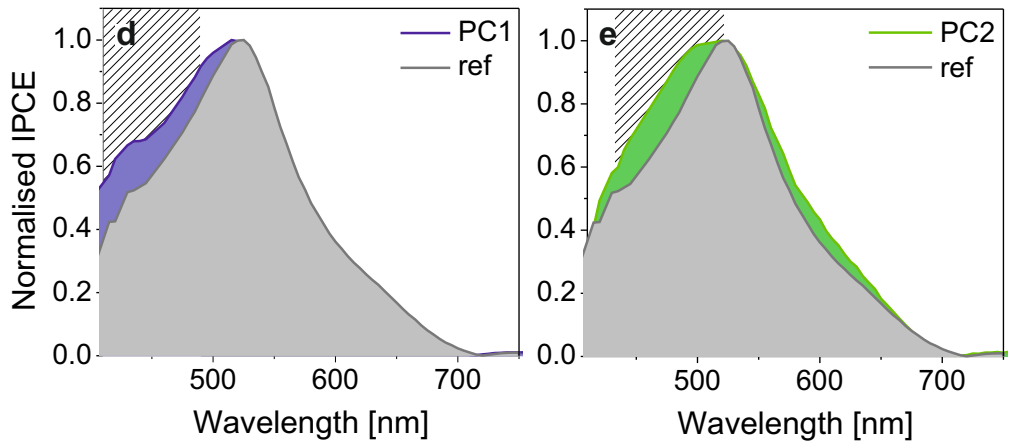

Wavelength [nm]

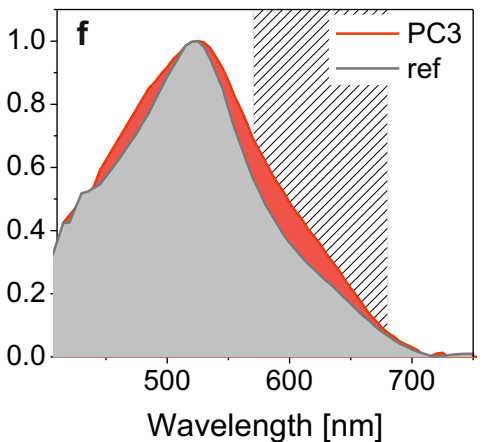

Figure 9.4: Incident-photon-to-electron conversion efficiency. a) - c) IPCE of devices with photonic crystal top layers compared to a single layer reference device. The available surface area of devices a) PC1 (blue) and c) PC3 (red) are comparable to the surface area of a $900 \mathrm{~nm}$ thick mesoporous single layer device (black). b) PC2 (green) has a PC of double thickness coupled to the $465 \mathrm{~nm}$ thick underlayer and therefore significantly more surface area. d) - f) Relative spectral IPCE of the different cell types normalised at a wavelength of $520 \mathrm{~nm}$. The hatched area illustrates the active region of the $3 \mathrm{D} \mathrm{PC}\left(n_{\mathrm{TiO} 2}=2.3, n_{\mathrm{elec}}=1.34\right)$.

$550 \mathrm{~nm}$ and $650 \mathrm{~nm}$ in PC2 can be related to the increased overall surface area of the device as compared to PC1, PC3 and the reference cell. Due to the poor absorption of the dye in this wavelength range, an increased film thickness will lead to a relative enhancement of the IPCE in this region [29]. In contrast, the substantially increased IPCE in the red for the PC3 cell in Figure 9.4 arises from the PC resonance at $625 \mathrm{~nm}$. Finally, the enhancement is found in a wider spectral region than is expected in the case of slow photon propagation modes at the red edge of the photonic band gap [15].

These results are in agreement with a recent experimental study by Lee et al. who identified three main effects for the increase in photocurrent in double layer DSCs [23]: (I) multidirectional scattering of short wavelength photons due to defects in the PC layer [12], (II) back reflection of light in the stop band region of the PC due to the PC layer acting as a dielectric mirror, and (III) an absorption enhancement caused by resonant modes as predicted by Mihi and Míguez [18]. Lee et al.'s approach of adding a photonic crystal top layer resulted, however, only in a weak correlation between the absorption properties of the device and the optical 
properties of the 3D PC. They assigned this finding to a lack of physical contact between the layers and a rough interface [23]. In contrast, the herein presented fabrication method results in a close contact and a smooth interface between the mesoporous $\mathrm{TiO}_{2}$ and the photonic crystal. This enables a significant enhancement in specific parts of the action spectrum as well as additional charge carrier generation arising from the photoactive material of the photonic crystal, which is now a functional part of the DSC.

Figure 9.5a shows a schematic of a DSC based on the self-assembled double layer photoanode. Current-voltage $(J-V)$ measurements of the cell types were carried out under standardised AM 1.5 illumination of $100 \mathrm{~mW} / \mathrm{cm}^{2}$ (active area $0.13 \mathrm{~cm}^{2}$, spectral mismatch $22 \%$ ). In Figure 9.5b the $J-V$ curves are shown for the double layer PC3 device in comparison to a reference cell. The PC3 exhibited a $10 \%$ increase in photocurrent compared to the reference cell, with $J_{\mathrm{sc}}=4.7 \mathrm{~mA} / \mathrm{cm}^{2}$. This is in accordance to the IPCE measurements shown in Figure 9.4c. The double layer device featured, however, a slightly lower open circuit potential $V_{\text {oc }}$ $(0.74 \mathrm{~V} v s .0 .77 \mathrm{~V})$ and a $15 \%$ decrease in fill factor $F F(0.52$ vs. 0.61). As a consequence, the overall conversion efficiency $\eta$ of $1.8 \%$ was $0.2 \%$ lower than for the reference cell. Míguez and coworkers recently pointed out that very thick inverse opal films $(>5 \mu \mathrm{m})$ are likely to have an adverse effect on charge transport and recombination through the cell, which may result in a penalty in photovoltage and photocurrent under real operation conditions [13]. Device fabrication has not been optimised in this study and only served as a proof of principle. Therefore, this hypothesis can be neither rejected not supported. Meanwhile other groups have further developed the presented concept and achieved efficiencies of up to $4.6 \%$ [30, 31, 32]. Fabrication techniques for the assembly of a defect-free 3D PCs made of $\mathrm{TiO}_{2}$ have evolved $[33,34]$, and studies on the development of $\mathrm{BCP}$-derived $\mathrm{TiO}_{2}$ films have led to the forma-
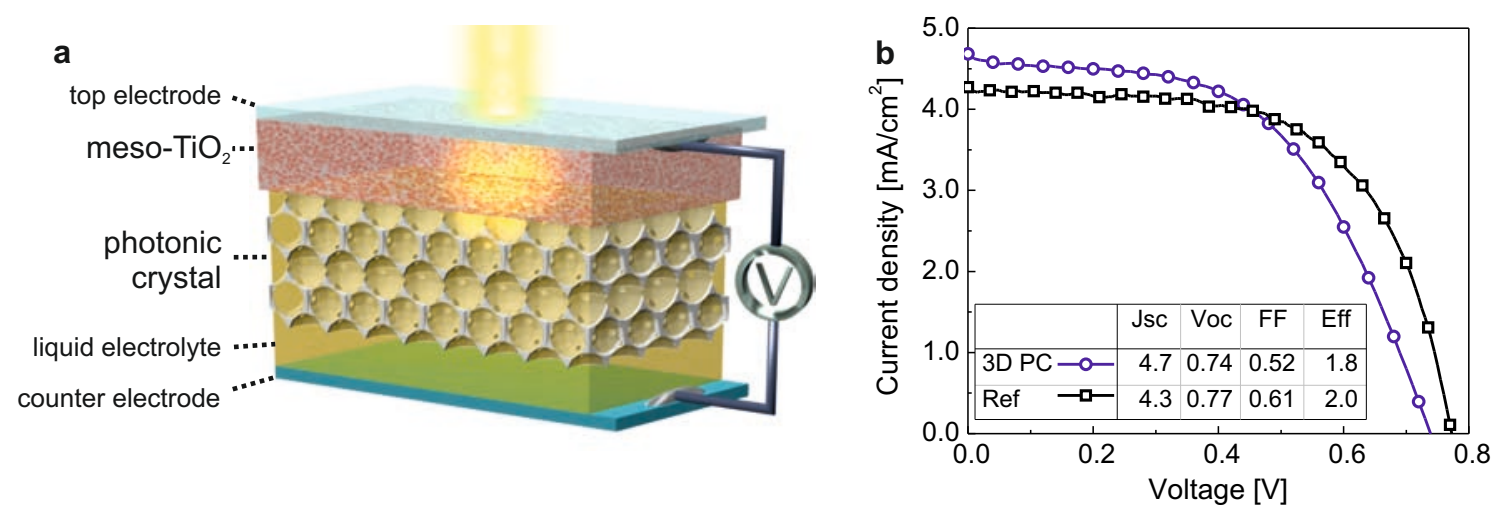

Figure 9.5: Device schematic and $J-V$ characteristics. a) Schematic of a fully assembled DSC based on the self-assembled double layer photoanode. b) $J-V$ characteristics of the double layer device PC3 (blue) and a reference single layer cell of similar internal surface area (black) under standardised AM 1.5 illumination. 
tion of significantly thicker crack-free films [35] (see Chapter 6). These advances may soon lead to further overall efficiency improvements. As an alternative concept to a 3D PC, the integration of a 1D PC in form of an mesoporous Bragg reflector (MDBR) has recently been proposed with reported efficiencies of up to $7.0 \%[13,36]$. MDBRs typically exhibit a broader stop-band and promise a less elaborate fabrication. Certainly the approach of BCP-directed, tunable MDBRs, presented in Chapter 8, is viable to improve this concept. The fully electronconducting architecture would enable light absorption and charge generation by the optical element, thus integrating the 1D PC as an active component in the device. Another promising concept is to couple PC-based reflectors behind the counterelectrode, where they are not an electronically active part of the device but enable effective light management and redistribution of non-absorbed photons. A recent study by Hwang et al. emphasises that non-absorbed light can be better redistributed in the device by a coherent diffraction element such as a $3 \mathrm{D}$ PC in comparison to a metallic backreflector or a scattering layer [37]. Comparing these three optical elements to a reference DSC, they found an overall conversion enhancement of $14 \%$ for a 3D PC, compared to $4.7 \%$ when using a metal back reflector and $2.1 \%$ for a scattering layer. This lead to an overall power conversion efficiency of $12.2 \%$ for PC-based DSCs.

\subsection{Conclusions}

In conclusion, a fabrication method for a $\mathrm{TiO}_{2}$ electrode architecture is presented which couples an optically and electrically active 3D PC top layer to a high surface area mesoporous underlayer. In contrast to earlier studies, the double layer structure exhibits porosity at the mesoporous and the microporous length scales as well as pore and electronic connectivity at all levels. This construct enables effective dye sensitisation, electrolyte infiltration and charge collection from both the mesoporous and the PC layers. Due to the smooth layer interface and the direct physical and electronic contact between the layers, light harvesting in specific parts of the spectrum was significantly increased by exploiting PC-induced resonances. Further investigation of its device photovoltaic characteristics, including charge mobility and recombination rates as well as optimisation of the PC structure are needed to fully exploit the IPCE increase. This is the first approach to fully integrate a PC top layer into a functioning DSC, opening an additional parameter space for effective light management. Finally, since the integration of an electrically and optically active PC increases the absolute IPCE and broadens the light harvesting capability of DSCs, this approach should be useful in solid state devices where pore infiltration is a limiting factor $[38,39]$ as well as in weakly absorbing organic photovoltaic devices [40]. 


\section{Bibliography}

[1] S. Guldin, S. Hüttner, M. Kolle, M. Welland, P. Müller-Buschbaum, R. Friend, U. Steiner, and N. Tetreault, "Dye-sensitized solar cell based on a three-dimensional photonic crystal," Nano Letters, vol. 10, no. 7, pp. 2303-2309, 2010.

[2] S. Guldin, P. Docampo, S. Hüttner, P. Kohn, M. Stefik, S. H.J., U. Wiesner, and U. Steiner, "Self-assembly as a design tool for the integration of photonic structures into excitonic solar cells," Proceedings of the SPIE, vol. 8111, DOI: 10.1117/12.893798, 2011.

[3] B. O'Regan and M. Grätzel, "A low-cost, high-efficiency solar-cell based on dyesensitized colloidal $\mathrm{TiO}_{2}$ films," Nature, vol. 353, no. 6346, pp. 737-740, 1991.

[4] J. Kroon, N. Bakker, H. Smit, P. Liska, K. Thampi, P. Wang, S. Zakeeruddin, M. Grätzel, A. Hinsch, S. Hore, U. Würfel, R. Sastrawan, J. Durrant, E. Palomares, H. Pettersson, T. Gruszecki, J. Walter, K. Skupien, and G. Tulloch, "Nanocrystalline dye-sensitized solar cells having maximum performance," Progress In Photovoltaics, vol. 15, no. 1, pp. 1-18, 2007.

[5] Z. Wang, H. Kawauchi, T. Kashima, and H. Arakawa, "Significant influence of $\mathrm{TiO}_{2}$ photoelectrode morphology on the energy conversion efficiency of N719 dye-sensitized solar cell," Coordination Chemistry Reviews, vol. 248, no. 13-14, pp. 1381-1389, 2004.

[6] J.-H. Yum, E. Baranoff, S. Wenger, M. Nazeeruddin, and M. Grätzel, "Panchromatic engineering for dye-sensitized solar cells," Energy $\mathcal{E}$ Environmental Science, vol. 4, no. 3, pp. 842-857, 2011.

[7] J. Ferber and J. Luther, "Computer simulations of light scattering and absorption in dyesensitized solar cells," Solar Energy Materials And Solar Cells, vol. 54, no. 1-4, pp. 265$275,1998$.

[8] S. Hore, C. Vetter, R. Kern, H. Smit, and A. Hinsch, "Influence of scattering layers on efficiency of dye-sensitized solar cells," Solar Energy Materials and Solar Cells, vol. 90, no. 9, pp. 1176-1188, 2006.

[9] Y. Chiba, A. Islam, Y. Watanabe, R. Komiya, N. Koide, and L. Han, "Dye-sensitized solar cells with conversion efficiency of 11.1\%," Japanese Journal Of Applied Physics Part 2-Letters $\mathcal{E}$ Express Letters, vol. 45, no. 24-28, pp. L638-L640, 2006.

[10] M. Nazeeruddin, T. Bessho, L. Cevey, S. Ito, C. Klein, F. De Angelis, S. Fantacci, P. Comte, P. Liska, H. Imai, and M. Grätzel, "A high molar extinction coefficient charge transfer sensitizer and its application in dye-sensitized solar cell," Journal Of Photochemistry And Photobiology A-Chemistry, vol. 185, no. 2-3, pp. 331-337, 2007.

[11] S. Nishimura, N. Abrams, B. Lewis, L. Halaoui, T. Mallouk, K. Benkstein, J. van de Lagemaat, and A. Frank, "Standing wave enhancement of red absorbance and photocurrent in dye-sensitized titanium dioxide photoelectrodes coupled to photonic crystals," Journal of the American Chemical Society, vol. 125, no. 3, pp. 6306-6310, 2003.

[12] L. Halaoui, N. Abrams, and T. Mallouk, "Increasing the conversion efficiency of dyesensitized $\mathrm{TiO}_{2}$ photoelectrochemical cells by coupling to photonic crystals," Journal of Physical Chemistry B, vol. 109, no. 13, pp. 6334-6342, 2005. 
[13] S. Colodrero, A. Mihi, L. Haggman, M. Ocaña, G. Boschloo, A. Hagfeldt, and H. Míguez, "Porous one-dimensional photonic crystals improve the power-conversion efficiency of dye-sensitized solar cells," Advanced Materials, vol. 21, no. 7, p. 764770, 2009.

[14] D. Colonna, S. Colodrero, H. Lindstrom, A. Di Carlo, and H. Míguez, "Introducing structural colour in dscs by using photonic crystals: interplay between conversion efficiency and optical properties," Energy $\mathcal{F}$ Environmental Science, DOI: 10.1039/C2EE02658A, 2012.

[15] K. Sakoda, "Enhanced light amplification due to group-velocity anomaly peculiar to twoand three-dimensional photonic crystals," Optics Express, vol. 4, no. 5, pp. 167-176, 1999.

[16] D. Mittleman, J. Bertone, P. Jiang, K. Hwang, and V. Colvin, "Optical properties of planar colloidal crystals: dynamical diffraction and the scalar wave approximation," Journal of Chemical Physics, vol. 111, no. 1, pp. 345-354, 1999.

[17] R. Rengarajan, D. Mittleman, C. Rich, and V. Colvin, "Effect of disorder on the optical properties of colloidal crystals," Physical Review E, vol. 71, no. 1, Part 2, pp. 1596815976, 2005.

[18] A. Mihi and H. Míguez, "Origin of light-harvesting enhancement in colloidal-photoniccrystal-based dye-sensitized solar cells," Journal Of Physical Chemistry B, vol. 109, no. 33, pp. 15968-15976, 2005.

[19] S. Ito, S. Zakeeruddin, P. Comte, P. Liska, D. Kuang, and M. Grätzel, "Bifacial dyesensitized solar cells based on an ionic liquid electrolyte," Nature Photonics, vol. 2, no. 11, pp. 693-698, 2008.

[20] A. Mihi, F. Lopez-Alcaraz, and H. Míguez, "Full spectrum enhancement of the light harvesting efficiency of dye sensitized solar cells by including colloidal photonic crystal multilayers," Applied Physics Letters, vol. 88, no. 19, p. 193110, 2006.

[21] R. Pozas, A. Mihi, M. Ocana, and H. Míguez, "Building nanocrystalline planar defects within self-assembled photonic crystals by spin-coating," Advanced Materials, vol. 18, no. 9, pp. 1183-1187, 2006.

[22] A. Mihi, M. E. Calvo, J. Anta, and H. Míguez, "Spectral response of opal-based dyesensitized solar cells," Journal Of Physical Chemistry C, vol. 112, no. 1, pp. 13-17, 2008.

[23] S.-H. A. Lee, N. Abrams, P. Hoertz, G. Barber, L. Halaoui, and T. Mallouk, "Coupling of titania inverse opals to nanocrystalline titania layers in dye-sensitized solar cells," Journal Of Physical Chemistry B, vol. 112, no. 46, pp. 14415-14421, 2008.

[24] M. Nedelcu, S. Guldin, M. Orilall, J. Lee, S. Hüttner, E. Crossland, S. Warren, C. Ducati, P. Laity, D. Eder, U. Wiesner, U. Steiner, and H. Snaith, "Monolithic route to efficient dye-sensitized solar cells employing diblock copolymers for mesoporous $\mathrm{TiO}_{2}$," Journal Of Materials Chemistry, vol. 20, no. 7, pp. 1261-1268, 2010.

[25] P. Jiang, J. Bertone, K. Hwang, and V. Colvin, "Single-crystal colloidal multilayers of controlled thickness," Chemistry Of Materials, vol. 11, no. 8, pp. 2132-2140, 1999. 
[26] H. Míguez, G. Ozin, S. Yang, and N. Tetreault, "Mechanical stability enhancement by pore size connectivity control in colloidal crystals by layer-by-growth of oxide," US Patent App. 11/878,023, 2007.

[27] S. Guldin, "Nanostructuring inorganic material by copolymer-assisted self-assembly and its multifunctional use for dye-sensitised solar cells," Master's thesis, Technische Universität München, 2008.

[28] A. Usami, "Theoretical study of application of multiple scattering of light to a dye-sensitized nanocrystalline photoelectrochemical cell," Chemical Physics Letters, vol. 277, no. 1-3, pp. 105-108, 1997.

[29] M. Nazeeruddin, A. Kay, I. Rodicio, R. Humphry-Baker, E. Muller, P. Liska, N. Vlachopoulos, and M. Grätzel, "Conversion of light to electricity by cis-x2bis(2,2'bipyridyl-4,4'-dicarboxylate)ruthenium(ii) charge-transfer sensitizers (X $=\mathrm{Cl}-, \mathrm{Br}-, \mathrm{I}-$, Cn-, and Scn-) on nanocrystalline $\mathrm{TiO}_{2}$ electrodes," Journal Of The American Chemical Society, vol. 115, no. 14, pp. 6382-6390, 1993.

[30] Y. Seo, K. Woo, J. Kim, H. Lee, and W. Lee, "Rapid fabrication of an inverse opal $\mathrm{TiO}_{2}$ photoelectrode for DSSC using a binary mixture of $\mathrm{TiO}_{2}$ nanoparticles and polymer microspheres," Advanced Functional Materials, vol. 21, no. 16, pp. 3094-3103, 2011.

[31] A. Mihi, C. Zhang, and P. Braun, "Transfer of preformed three-dimensional photonic crystals onto dye-sensitized solar cells," Angewandte Chemie - Int. Ed., vol. 50, no. 25, pp. 5711-5714, 2011.

[32] J.-H. Shin and J. Moon, "Bilayer inverse opal $\mathrm{TiO}_{2}$ Electrodes for dye-sensitized solar cells via post-treatment," Langmuir, vol. 27, no. 10, pp. 6311-6315, 2011.

[33] B. Hatton, L. Mishchenko, S. Davis, K. Sandhage, and J. Aizenberg, "Assembly of large-area, highly ordered, crack-free inverse opal films," Proceedings Of The National Academy Of Sciences Of The United States Of America, vol. 107, no. 23, pp. 1035410359, 2010.

[34] L. Liu, S. Karuturi, L. Su, and A. I. Y. Tok, "TiO ${ }_{2}$ inverse-opal electrode fabricated by atomic layer deposition for dye-sensitized solar cell applications," Energy $\mathcal{E}$ Environmental Science, vol. 4, no. 1, pp. 209-215, 2011.

[35] S. Guldin, P. Docampo, M. Stefik, G. Kamita, U. Wiesner, H. Snaith, and U. Steiner, "Layer-by-layer formation of block copolymer derived $\mathrm{TiO}_{2}$ for solid state dye-sensitized solar cells," Small, vol. 8, no. 3, pp. 432-440, 2012.

[36] S. Colodrero, A. Forneli, C. Lopez-Lopez, L. Pelleja, H. Míguez, and E. Palomares, "Efficient Transparent Thin Dye Solar Cells Based on Highly Porous 1D Photonic Crystals," Advanced Functional Materials, vol. 22, no. 6, pp. 1303-1310, 2012.

[37] D.-K. Hwang, B. Lee, D.-H. Kim, and R. Chang, "High-efficiency dye-sensitized solar cells using 3D photonic crystals," submitted.

[38] U. Bach, D. Lupo, P. Comte, J. E. Moser, F. Weissörtel, J. Salbeck, H. Spreitzer, and M. Grätzel, "Solid-state dye-sensitized mesoporous $\mathrm{TiO}_{2}$ solar cells with high photonto-electron conversion efficiencies," Nature, vol. 395, pp. 583-585, 1998. 
[39] J. Melas-Kyriazi, I.-K. Ding, A. Marchioro, A. Punzi, B. Hardin, G. Burkhard, N. Tetreault, M. Grätzel, J.-E. Moser, and M. McGehee, "The effect of hole transport material pore filling on photovoltaic performance in solid-state dye-sensitized solar cells," Advanced Energy Materials, vol. 1, no. 3, pp. 407-414, 2011.

[40] J. Halls, C. Walsh, N. Greenham, E. Marseglia, R. Friend, S. Moratti, and A. Holmes, "Efficient photodiodes from interpenetrating polymer networks," Nature, vol. 376, no. 6540, pp. 498-500, 1995. 


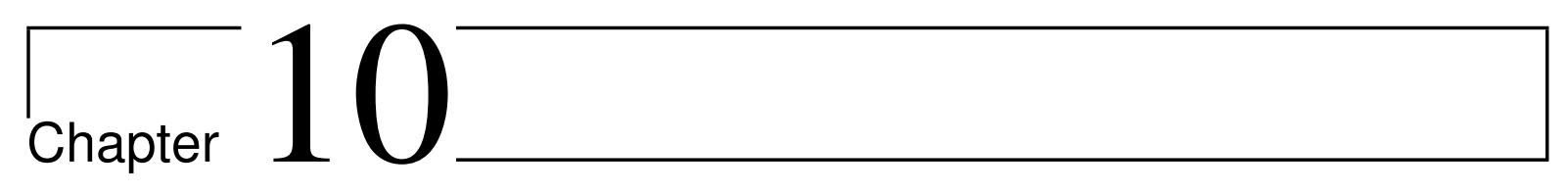

\title{
Block copolymer assembled antireflective coatings with self-cleaning properties
}

\author{
Work in preparation for publication by Stefan Guldin et al. [1]
}

\subsection{Introduction}

Since Fraunhofer's [2] and Lord Rayleigh's [3] first experimental approaches to reduce light reflection off optical interfaces, antireflective coatings (ARCs) have been in the constant focus of scientific and industrial interest. While the underlying physical origin is long understood, the implementation of broadband antireflective coatings on low refractive index substrates remains a challenge. Antireflection (ar) is typically achieved by destructive interference of light reflected from the two (or more) interfaces of a thin film optical coating (schematic in Figure 10.1). Two conditions have to be fulfilled for a single layer ARC: (1) Phase matching requires an optical layer thickness $h_{\text {ar }}$ of one quarter of the wavelength of the incident light $\lambda$ : $n_{\mathrm{ar}} h_{\mathrm{ar}}=1 / 4 \lambda$. (2) Complete destructive interference further depends on amplitude matching of the reflected beams, i.e. $n_{\mathrm{ar}}=\sqrt{n_{0} n_{\mathrm{s}}}$, with $n_{\mathrm{ar}}, n_{0}, n_{\mathrm{s}}$ as the refractive indices of the ARC, the medium of the incident beam and the substrate, respectively [4]. While the first condition is easily met with modern deposition techniques, the second requirement poses a challenge. Many transparent substrate materials have a refractive index of around 1.5, thus requiring $n_{\mathrm{ar}} \sim 1.22$. For thin film processing, magnesium fluoride with $n \sim 1.37$ is one of the lowestindex materials, which fulfills the second condition only for high refractive index substrates $\left(n_{\mathrm{s}} \sim 1.9\right)$ and yields a much lower optical performance when used as coating for common glasses and transparent plastics [4]. A further decrease in refractive index can only be achieved by the introduction of porosity on the sub-optical length scale. The effective refractive index 
is then a direct consequence of the material-air composite and can be approximated by various effective medium theories, such as the Bruggeman model [5, 6, 7] (see Chapter 2.5).

Phase separation on the nanometre scale with subsequent removal of one sacrificial phase is a practical route to ultralow refractive index layers. This concept was established by Walheim et al. for nanophase separated polymer films [8] and subsequently extended to mesoporous inorganic coatings [9]. Other methods to prepare low refractive index layers by solution processing include the controlled assembly of colloids by electrostatic attraction [10] and the layer-by-layer deposition of $\mathrm{SiO}_{2}$ nanoparticle films [11]. Gombert et al. introduced an antireflective film based on the replication of a sub-wavelength surface relief from a master template by nanoimprint-lithography [12]. Vacuum-based fabrication methods for ultralow refractive index layers on glass include glancing angle and chemical vapor deposition [13, 14].

An alternative concept to antireflection takes inspiration from the nanostructured topography of the corneal lenses, found in moth eyes $[15,16]$. Cone-like structural features arranged on a hexagonal grid with a lattice constant below the diffraction limit give rise to a continuous refractive index gradient between air and substrate, therefore removing the sharp optical interface. This was initially realised for microwave frequencies by standard photolithography [17]. Recently, reactive ion etching using colloidal masks [18] or a metal-particle containing resist [19] allowed access to sub-100 $\mathrm{nm}$ feature sizes and therefore reduced light reflection in the visible spectrum. Although this is a promising concept, the necessary fabrication routes are elaborate and yield so far no competitive optical response compared to interference-based ARCs.

While research-grade nanostructured ARCs are close to perfection, their implementation in commercial products is hampered by their lack of wear resistance and optical variability caused by contamination of the composite network. In particular for outdoor applications, ARCs need to be structurally resistant and should recover from ambient pollution. The latter can in principle be implemented through self-cleaning based on surface superhydrophobicity or photocatalysis [20]. Superhydrophobic surfaces are self-cleaning in a sense that particulate contaminants adhere only very weakly and are easily washed off by water. Photocatalytic coatings, on the other hand, do not rely on a cleaning medium, but decompose organic contaminants by light induced redox-reactions. While photocatalytic self-cleaning is in principle more robust, the inclusion of a photocatalytic component in ARCs, typically $\mathrm{TiO}_{2}$, poses a major challenge because of the high refractive index of $n_{\mathrm{TiO}_{2}}>2.5$. Several concepts have been proposed to combine self-cleaning and antireflection, including the surface coating of a colloidal-based ARCs with $\mathrm{TiO}_{2}$ [21], the co-deposition of $\mathrm{TiO}_{2}$ and $\mathrm{SiO}_{2}$ nanoparticles to form a porous ARC [11] and a double layer structure of low refractive index $\mathrm{SiO}_{2}$ and $\mathrm{TiO}_{2}$ $[22,23]$. All of these approaches require a high-temperature processing step, which prevents 
their use on flexible, plastic-based substrates. Furthermore, the structural arrangement on the nanometre-scale limits the achievable porosity and thus, the volume fraction of $\mathrm{TiO}_{2}$ that can be incorporated without compromising on the resulting effective refractive index.

In this chapter a new concept is presented towards the combination of antireflection and photocatalysis, which relies on the use of a high molecular weight PI- $b$-PEO block copolymer (BCP) in combination with silica-based sol-gel chemistry. The approach makes use of the micellisation of the hybrid system under non-equilibrium processing conditions, as discussed in Chapter 5. Upon spin coating, the sacrificial colloidal micelles arrange in a face-centred cubic (FCC) geometry, thereby co-assembling the inorganic material in an inverse-opal like morphology of ultralow refractive index. Thus, the approach allows for partial substitution of silica-type material with high refractive index $\mathrm{TiO}_{2}$ nanocrystals. The interplay $\mathrm{TiO}_{2}$ incorporation and resulting self-cleaning properties is investigated as well as the optical performance of the coatings and their processing on a variety of substrates.

\subsection{Experimental}

\section{ARC fabrication}

Two different PI- $b$-PEO copolymers were used as structure-directing agents in this study: I) BCP-34: $M_{\mathrm{n}}=34.4 \mathrm{~kg} / \mathrm{mol}, 28 \mathrm{wt} \%$ PEO and II) BCP-92: $M_{\mathrm{n}}=91.6 \mathrm{~kg} / \mathrm{mol}, 28.6 \mathrm{wt} \%$ PEO. $\mathrm{TiO}_{2}$ nanocrystal synthesis was carried out by Dr. Peter Kohn, using a similar method as reported in reference [24]. The resulting wet precipitate was dried for two hours in ambient conditions and then redissolved in an azeotrope solvent mixture of toluene (72.84 wt $\%)$ and 1-butanol (27.16 wt\%). To maintain consistent concentrations of $\mathrm{TiO}_{2}$ nanocrystals in the azeotrope solution ( $20 \mu \mathrm{g}$ per $\mathrm{ml}$ ), a fraction of the precipitate was fully dried and heated to $350{ }^{\circ} \mathrm{C}$ aside in order to reveal the weight content of $\mathrm{TiO}_{2}$. The aluminosilicate sol was prepared as described in Chapter 5.2. Three different hybrid solutions were created. For $25 \mathrm{wt} \%$ $\mathrm{TiO}_{2}$ loading, $50 \mathrm{mg}$ polymer was dissolved in $0.7 \mathrm{ml}$ of azeotrope solvent and a further $0.5 \mathrm{ml}$ of $\mathrm{TiO}_{2}$ solution, before $56 \mathrm{mg}$ aluminosilicate sol was added. For $37.5 \mathrm{wt} \% \mathrm{TiO}_{2}$ loading, $50 \mathrm{mg}$ polymer was dissolved in $0.5 \mathrm{ml}$ of azeotrope solvent and $0.7 \mathrm{ml}$ of $\mathrm{TiO}_{2}$ solution, before $47 \mathrm{mg}$ sol was blended in. A $50 \mathrm{wt} \% \mathrm{TiO}_{2}$ solution was prepared by adding $50 \mathrm{mg}$ polymer to a mixture of $0.6 \mathrm{ml}$ azeotrope solvent and $0.94 \mathrm{ml} \mathrm{TiO}_{2}$ solution, before $37.5 \mathrm{mg}$ sol was added. The hybrid solutions were then stirred for one hour and further diluted as needed to match the required film thicknesses. Hybrid films were deposited onto pre-cleaned glass slides by spin coating $(2000 \mathrm{rpm}, 20 \mathrm{~s})$. The cast films were annealed on a hotplate by gradual increasing the temperature to $200{ }^{\circ} \mathrm{C}$ (180 min linear ramp, $30 \mathrm{~min}$ dwell time). Finally, the organic 
component of the hybrid films was removed by reactive ion etching in oxygen plasma (30 min, $100 \mathrm{~W}, 0.33$ mbar, STS Instruments, 320PC RIE). Because of the limited temperature stability of the PET (polyethylene terephthalate) substrates, thermal annealing of ARCs on flexible substrates was reduced to $130{ }^{\circ} \mathrm{C}$ ( 15 min linear ramp, 5 min dwell time), before the substrates were similarly exposed to 30 min oxygen plasma. For flexible substrates, a aluminium sample holder was built to allow double-sided coating.

\section{ARC characterisation}

Optical characterisation was carried out on a Olympus BX51 microscope with $5 \times$ magnification, where a $600 \mu \mathrm{m}$ fibre (Ocean Optics "QP600-2-SR”) and a high resolution spectrometer (Ocean Optics "QE65000”) were coupled into the beam path. Determination of optical constants was performed on a spectroscopic ellipsometer (Wollam "alpha-SE"), and the data was fitted with the manufacturer's software. For determination of the photocatalytic activity, stearic acid (analytical standard) was deposited by spin coating of a $8.8 \mathrm{mM}$ solution in methanol (1500 rpm, $20 \mathrm{~s}$ ). A xenon lamp (Bentham "IL75E", 75W) was used to simulate the solar irradiation in the ultra-violet spectral region. The absolute power density of the laboratory light source in the $275-375 \mathrm{~nm}$ spectral band, i.e. where $\mathrm{TiO}_{2}$ absorbs, was adjusted to the integrated value of the solar AM 1.5 spectrum $\left(2.89 \mathrm{~mW} / \mathrm{cm}^{2}\right)$. The uv irradiation intensity was determined using a power meter (Spectra Physics "Mod 407A") with bolometer head and a UV bandpass filter (Thorlabs "FGUV11") [25]. The photocatalytic decomposition of stearic acid was monitored by periodic FTIR measurements (Thermo Scientific "Nicolet iS10").

\subsection{Results and discussion}

Figure 10.1 illustrates the materials route for ARC manufacture. A high molecular weight PI- $b$-PEO BCP was dissolved in an azeotrope mixture of toluene and 1-butanol. An silicabased sol was prepared separately by the stepwise hydrolysis of a silicon/aluminium alkoxide mix (9/1 molar ratio) [26]. $\mathrm{TiO}_{2}$ nanocrystals were synthesised via a non-hydrolytic sol-gel chemistry route [27] and compatibilised with the azeotrope solvent mixture. The components were subsequently combined in a organic to inorganic weight ratio of 4 to 3 with varying amount of $\mathrm{TiO}_{2}$. The hybrid solution could then be processed into a thin film by spin coating, dip coating or meniscus coating. Following a temperature annealing step to condense the inorganic matrix, the organic components were removed by reactive ion etching in an oxygen plasma. See the experimental Section 10.2 for further details. 
a

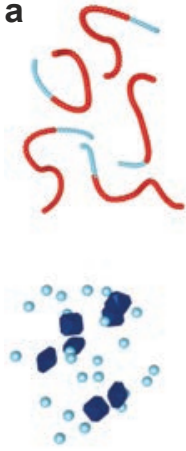

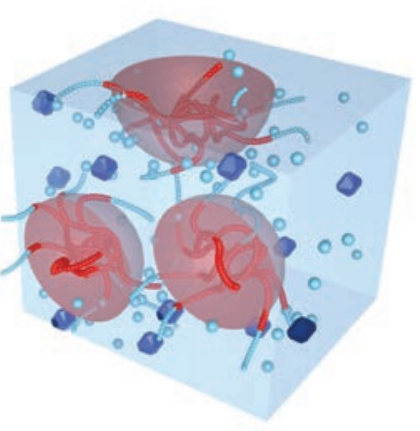

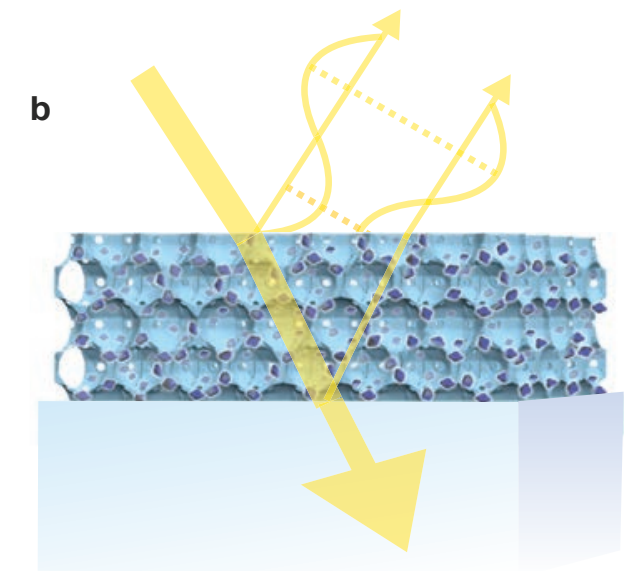

Figure 10.1: Schematic of ARC manufacture. a, A solution of PI- $b$-PEO block copolymer, silica-based sol and compatibilised $\mathrm{TiO}_{2}$ nanocrystals is used for the coating of transparent substrates. The inorganic components preferentially reside in the PEO phase and are therefore structure-directed during the micellar packing of the block copolymer. b, Subsequent reactive ion-etching in an oxygen plasma removes the polymer and reveals an inorganic inverse opal-type structure that is loaded with $\mathrm{TiO}_{2}$ nanocrystals. Phase and amplitude matching of the optical coating give rise to destructive interference of reflected light, i.e. enhanced transmission, while the embedded photocatalytic $\mathrm{TiO}_{2}$ nanocrystals prevent contamination of the ARC.

The resulting porous inorganic films are shown in Figure 10.2. Scanning electron microscopy (SEM) images show a network morphology that reveals its likely origin. The welldefined pore size and the local hexagonal arrangement is reminiscent of an inverse opal structure. The evolution of this morphology probably involved the formation of block-copolymer micelles in solution, which during solvent evaporation self-assembled into an opal morphology consisting of a PI core and a PEO+sol matrix. The condensation reaction and polymer removal then gives rise to the discernible network structure. Since the micellar size is determined by the polymer architecture, a variation of the solid organic to inorganic volume (or weight) fraction allows fine tuning the porosity, while affecting the pore size only very little. The interplay between mixing ratio and porosity is shown in Figure 10.2a-c, where the polymer weight fraction in the initial solution compared to the total weight of $\mathrm{BCP}$ and resulting silica-type material was increased from $33 \mathrm{wt} \%$ to $50 \mathrm{wt} \%$. The pore size of the inorganic network can be separately controlled by varying the molecular weight of the sacrificial PI block (see Chapter 5 for further details).

Figure $10.2 \mathrm{~d}$ shows the morphology of the film with similar inorganic loading as in Figure $10.2 \mathrm{c}$ but an increased molecular weight of the PI block. While a molecular weight of $24.8 \mathrm{~kg} / \mathrm{mol}$ led to a pore size of $\sim 33 \mathrm{~nm}$ (Figure 10.2a-c), increasing the PI chain length to $62.7 \mathrm{~kg} / \mathrm{mol}$ resulted in $\sim 53 \mathrm{~nm}$-wide pores. This is in good agreement with scaling laws governing polymer chains in a good solvent [28]. The radius of gyration of the pore forming 
PI block scales by a factor of 1.59 when increasing the molecular weight from $24.8 \mathrm{~kg} / \mathrm{mol}$ to $62.7 \mathrm{~kg} / \mathrm{mol}$. This is consistent with the pore size determination by SEM image analysis, shown previously in Chapter 5. The pore interconnectivity, arising from the close-packing of sacrificial micelles, can be clearly identified.

Spectroscopic ellipsometry of the resulting films revealed that the refractive index could be finely tuned in the range $1.40<n_{\mathrm{ar}}<1.13(\lambda=632 \mathrm{~nm})$ by varying the polymer weight fraction from $28 \%$ to $67 \%$ (see Chapter 5). The Bruggeman effective medium approximation for a network of air voids in an aluminosilicate matrix $(n=1.52)$ results in a porosity value $p \sim 73 \%$ for $n_{\mathrm{ar}}=1.13$. In conventional approaches to form mesoporous inorganic films, porosity arises from the interstitial space between packed silica nanoparticles or colloids. Random packing, i.e. jamming of monodisperse spheres leads to packing densities of $\sim 62-64 \%$, i.e. $p \sim 36-38 \%$ [29]. Experimentally, nanoparticle-based approaches readily achieve $p \sim 40 \%$ [30]. A further increase in pore volume may be realised by the introduction of a sacrificial spacer material, resulting in $p_{\max } \sim 50-60 \%$ [31]. The packing density of colloidal monolayers typically yields $p \sim 39 \%$ [32]. The origin of pore formation in the present route is fundamentally different to previous approaches as the pores arise from sacrificial micelles and not from the close-packing of high refractive index components. The inorganic material resides in the hydrophilic outer layer of the micelles and is therefore coassembled in an inverse-opal like geometry. As a consequence of the close-packing of pores, porosity values up $73 \%$ were reached after the removal of the BCP. Alternatively, high porosi-
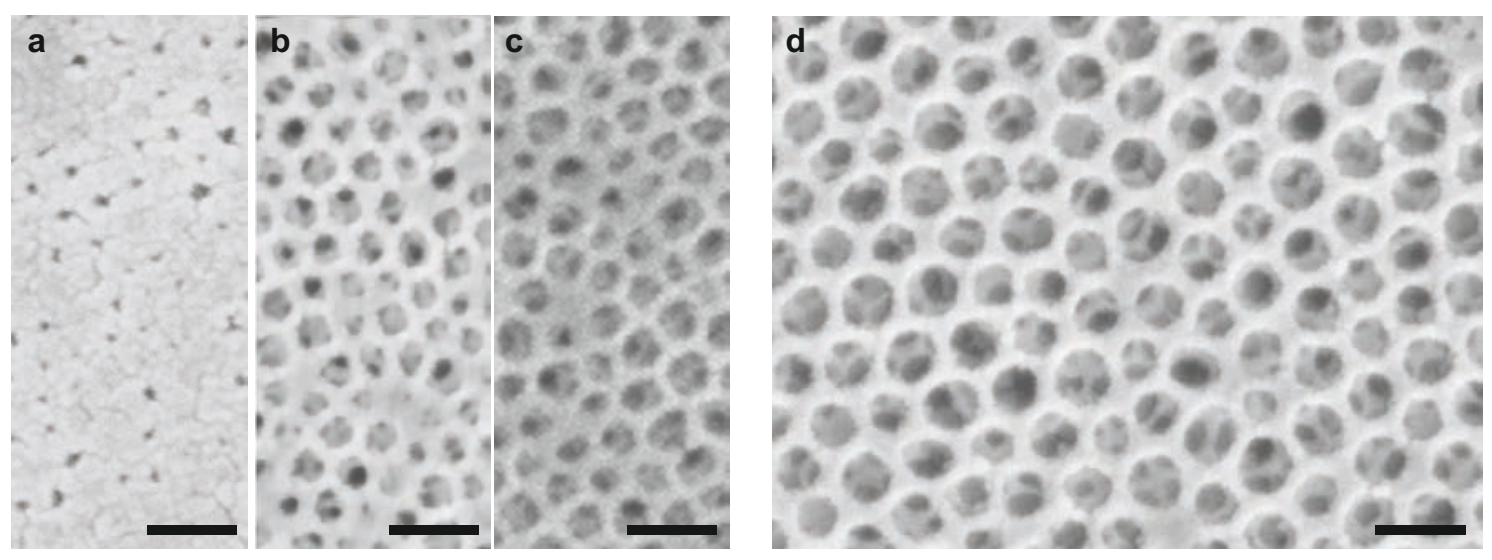

Figure 10.2: Film morphology of mesoporous optical coatings.a-c Scanning electron microscopy top-view of mesoporous silica-type films, stemming from a solution of BCP-34 of molecular weight $M_{\mathrm{n}}=34.4 \mathrm{~kg} / \mathrm{mol}$ and a polymer weight fraction of a: $33 \%, \mathbf{b}: 40 \%$ and c: $50 \%$, relative to the total weight of $\mathrm{BCP}$ in the initial solution and resulting silica-type material. $\mathbf{d}$ The use of a higher molecular weight polymer BCP-92 $\left(M_{\mathrm{n}}=91.6 \mathrm{~kg} / \mathrm{mol}\right)$, results in significantly larger pores and identical refractive index for otherwise similar conditions (here: $50 \%$ polymer weight content). The scale bars represent $100 \mathrm{~nm}$. 
ties can be achieved by sol infiltration into a BCP-assembled gyroid template [33], leading to a pore volume of $66 \%$. However, template formation is dependent on delicate structure control, which requires annealing of the films in a solvent-vapor atmosphere and film thicknesses above $130 \mathrm{~nm}$. In contrast, the pore volume in the presented method can be adjusted by place-holder micelles, where size and concentration can be easily controlled.

Adsorption of organic contaminants from the ambient atmosphere typically deteriorates the performance and long-term usability of mesoporous ARCs, particularly in outdoor applications. $\mathrm{TiO}_{2}$-based photocatalysis can be used to degrade the adsorbed hydrocarbons and restore ARC performance. In several previous studies the incorporation of photocatalytic $\mathrm{TiO}_{2}$ was reported, realised either by surface coating [21], blending [11] or in double layer stacks $[22,23]$. The amount of $\mathrm{TiO}_{2}$ that can be added to the ARC is, however, relatively small because of the resulting increase in refractive index. The presented approach leads to exceptionally low refractive index layers, which therefore allows to maximise $\mathrm{TiO}_{2}$ loading. This was achieved by the incorporation of crystalline 4-nm-sized $\mathrm{TiO}_{2}$ nanoparticles that were synthesised via a non-hydrolytic sol-gel chemistry route [27, 34].

The sol-gel approach was modified to compatibilise the nanoparticles with the solvent used in the polymer/silica-sol solution. The incorporation of already crystalline $\mathrm{TiO}_{2}$ has several advantages: No high-temperature step during the film fabrication is necessary to crystallise the $\mathrm{TiO}_{2}$. Furthermore, rather than forming a stoichiometric $\mathrm{Ti}_{x} \mathrm{Si}_{1-x} \mathrm{O}_{2}$ blend, which is often observed for a mix of silica and titania precursors [35], photoactive $\mathrm{TiO}_{2}$ hot spots are created within the network. While a post-treatment of $\mathrm{ARCs}$ with $\mathrm{TiO}_{2}$ precursor results in additional materials deposition, the present route enables substitutional incorporation. Figure 10.3 shows the variation of the ARC's refractive index as a function of the weight fraction of $\mathrm{TiO}_{2}$ nanocrystals in the initial hybrid solution. The refractive index scales with the replacement of alumunosilica by $\mathrm{TiO}_{2}$ according to the Bruggeman effective medium approximation $[5,7]$. Starting from a refractive index of $\sim 1.13$ for a mesoporous silica-type film without $\mathrm{TiO}_{2}$, this value increased to $n \sim 1.19 / 1.22 / 1.26$ when $25 / 27.5 / 50 \mathrm{wt} \%$ aluminosilicate in the initial solution was replaced by $\mathrm{TiO}_{2}$ nanocrystals. Thus, due to the $\sim 73 \%$ porosity of the inorganic network, up to $50 \mathrm{wt} \%$ aluminosilicate can be substituted by $\mathrm{TiO}_{2}$ while maintaining sufficiently low refractive indices.

The optical properties of the fabricated ARCs are presented in Figure 10.4. A comparison of non-catalytic films is shown Figure 10.4a. Porosity tuning by adjusting the inorganic to polymer mixing ratio (without the inclusion of $\mathrm{TiO}_{2}$ nanocrystals) allowed the fabrication of an silica-type film with $n \sim 1.22$ and $h \sim 110 \mathrm{~nm}$. When coated on both sides of a glass slide, the ARCs demonstrated their high optical quality with light transmittance $\mathcal{T}$ up to $99.8 \%$, shown in red. In direct comparison, the commercial benchmark for laboratory-use glass, a polymeric 


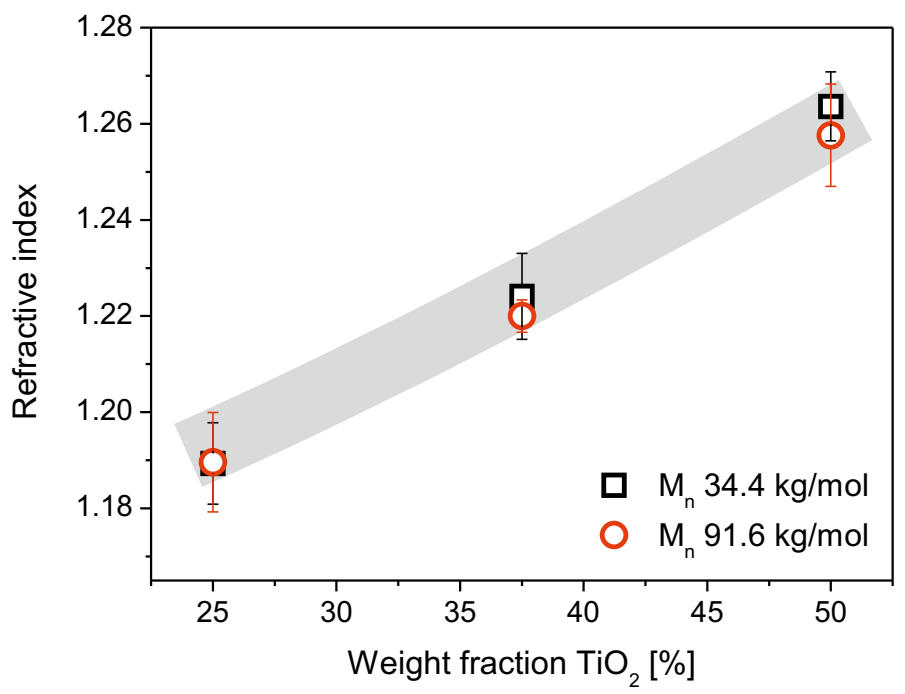

Figure 10.3: Refractive index as a function of $\mathbf{T i O}_{2}$ nanocrystal loading for mesoporous films derived from BCP-34 and BCP-92. The weight ratio between inorganic and organic constituents was kept constant at 3:4, while the aluminosilicate weight fraction in the initial solution was partially substituted by $\mathrm{TiO}_{2}$ nanocrystals. As a guideline, the expected scaling of the refractive index as a function of the $\mathrm{TiO}_{2}$ weight fraction, based on the Bruggeman effective medium approximation, is indicated in grey.

adhesive named "Arktop" (Asahi Glass) achieved a similarly high peak value $\mathcal{T}_{\max } \sim 99.5 \%$ (blue), but in a more limited spectral range. The transmittance of a reference glass slide without coating is shown in black. Figure $10.4 \mathrm{~b}$ displays the optical properties of a highly porous film, where $50 \mathrm{wt} \%$ aluminosilicate was substituted by photocatalytic $\mathrm{TiO}_{2}$ nanocrystals. Here, a maximum transmittance of $99.3 \%$ was measured. The inclusion of a photocatalytic materials into inorganic ARCs, using at low temperature protocol, makes the deposition technique attractive for the coating of flexible plastic substrates, such as PET (polyethylene terephthalate). In Figure 10.4c the effect of a double-sided photocatalytic coating onto the optical properties of a PET substrate is displayed. The sample exhibited a slightly lower peak transmittance of $98.8 \%$, which is on the other hand spectrally more broad band. PET is a promising substrate material for organic solar cells and flexible flat panel displays [36]. Material routes to ARCs on PET are challenged by the limited temperature and chemical stability of the substrate material. Hyun and coworkers reported the reduction of reflectance to $\mathcal{R}_{\min } \sim 1 \%$ by coating the substrate with a mesoporous silica film via a water-soluble structure directing agent [37]. Nanoimprinting of a sub-wavelength surface relief is another viable option, yielding transmittance values of $\mathcal{T}_{\max } \sim 98.3 \%(\lambda \sim 600 \mathrm{~nm})$ [38]. Yet, both approaches do not yield self-cleaning surfaces. The additional coating of the ARC with photocatalytic $\mathrm{TiO}_{2}$ results in a loss in transmittance of around $4-8 \%$ at each layer interface [39]. The presented approach 

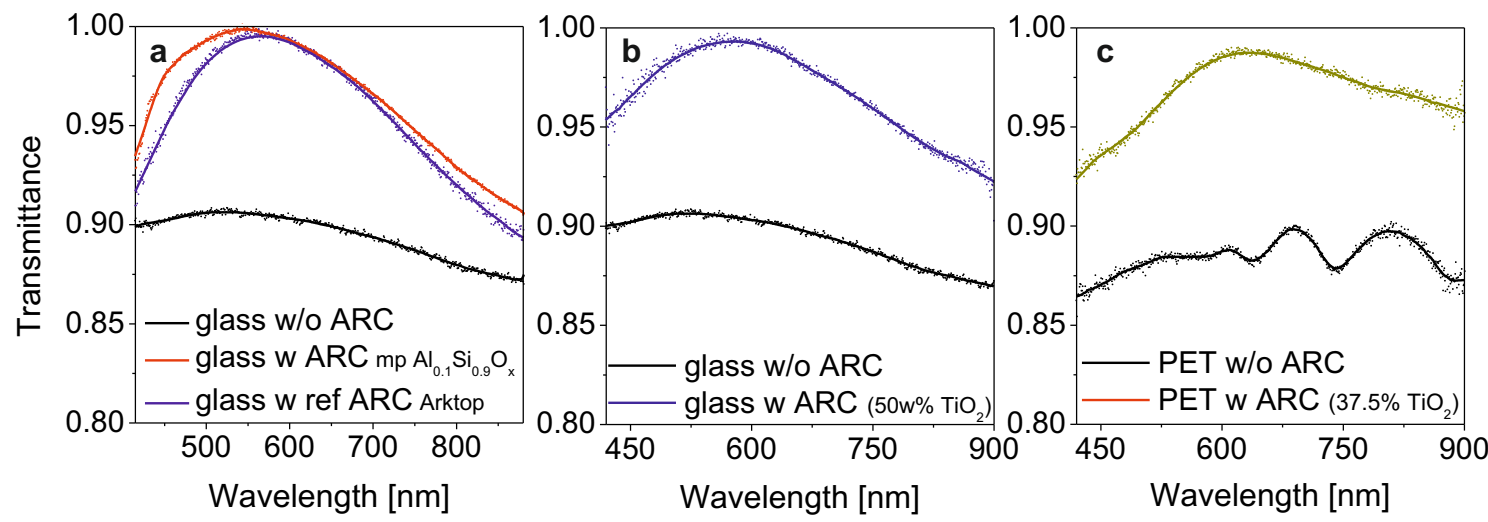

Figure 10.4: Transmission characteristics of antireflective coatings. a) Transmittance through an uncoated glass slide (black) in comparison to substrates coated with double-sided BCPassembled mesoporous ARC (red) or double-sided commercial antireflective adhesive (blue, Arktop). b) Optical transmittance of a glass slide with double-sided ARCs, where $50 \mathrm{wt} \%$ aluminosilicate was replaced by high refractive index, photocatalytic $\mathrm{TiO}_{2}$ nanocrystals. c) Optical properties of a PET substrate with and without double-sided coating of photocatalytic ARCs. All BCP-derived coatings were based on BCP-34.

therefore establishes a first practical route to antireflective coatings for PET substrates that are both photocatalytic and highly transmissive.

While the optical characterisation showed good uptake and sub-optical dispersion of $\mathrm{TiO}_{2}$ in the aluminosilicate network, the actual position of the nanocrystals is crucial for the selfcleaning function. Cluster formation or the inclusion of the $\mathrm{TiO}_{2}$ nanocrystals far from the network surface would be detrimental for their photocatalytic activity. Figure 10.5 shows the distribution of the $\mathrm{TiO}_{2}$ nanocrystals, as observed by transmission electron microscopy (TEM). The TEM images of two different pore morphologies show $\sim 3-4 \mathrm{~nm}$-sized nanocrystals that
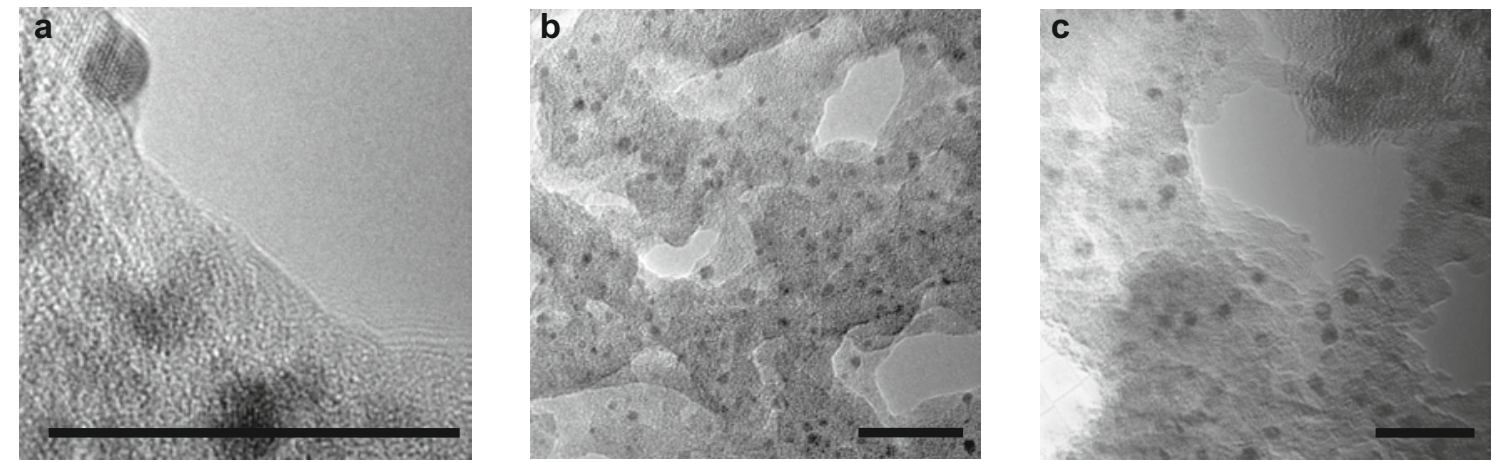

Figure 10.5: Composition of self-cleaning ARCs. Transmission electron micrographs showing the distribution of photocatalytic $\mathrm{TiO}_{2}$ nanocrystals in the inorganic network. a) High magnification image of $\mathrm{TiO}_{2}$ nanocrystals embedded in the silica-type matrix. Film morphology for b) BCP-34 and c) BCP-92 derived films. The scale bars represent $20 \mathrm{~nm}$. 
are well dispersed within the silica-based network. No $\mathrm{TiO}_{2}$ aggregates were found. This is supported by wide angle $\mathrm{x}$-ray diffraction results of dried $\mathrm{TiO}_{2}$ powder after non-hydrolytic sol-gel synthesis, where a mean crystallite size of $3.5 \pm 0.2 \mathrm{~nm}$ was determined by a Scherrer analysis of the [101] anatase peak [40].

The self-cleaning effect of photocatalytic $\mathrm{TiO}_{2}$ surfaces is based on the absorption of ultraviolet light in the $\mathrm{TiO}_{2}$. A small fraction of the generated charge carriers escapes rapid recombination and migrates to the $\mathrm{TiO}_{2}$ surface. Electron vacancies cause the oxidation of adsorbed organic molecules, while electrons eventually combine with atmospheric oxygen to form superoxide radicals, which further degrade nearby organic molecules. This so-called "cold combustion" mechanism enables self-cleaning through the conversion organic pollutants to carbon dioxide, water and mineral acids [20]. The decomposition of stearic acid is often used as an organic marker molecule to monitor the photocatalytic performance of selfcleaning surfaces [20, 41, 42]. Stearic acid readily assembles in a homogeneous layer onto inorganic surfaces. Its decomposition can be monitored by Fourier transform infrared spectroscopy (FTIR). As $\mathrm{TiO}_{2}$ only absorbs in the spectral range $\lambda<375 \mathrm{~nm}\left(E_{\text {gap }} \sim 3.3 \mathrm{eV}\right.$ [43]), it was of central importance to closely mimic the solar irradiation (AM 1.5) in the ultraviolet frequency range. This was achieved by a xenon lamp that was calibrated to match the intensity of ambient solar power in this spectral window.

Figure 10.6a-f shows the decomposition of stearic acid adsorbed onto ARCs for two different pore sizes (a-c: $33 \mathrm{~nm}$; d-f: $53 \mathrm{~nm}$ ), each with $\mathrm{TiO}_{2}$ loadings of $25-50 \mathrm{wt} \%$. FTIR absorbance spectra were collected in transmission and baseline corrected. In the spectral range from $2800-3000 \mathrm{~cm}^{-1}$ stearic acid shows three peaks: the asymmetric in-plane C-H methyl stretching results in absorbance at $2958 \mathrm{~cm}^{-1}$, while the $2923 \mathrm{~cm}^{-1}$ and $2853 \mathrm{~cm}^{-1}$ peaks correspond to symmetric and asymmetric $\mathrm{C}-\mathrm{H}$ stretching modes of $\mathrm{CH}_{2}$, respectively [42]. An integrated absorbance of $0.52 \mathrm{~cm}^{-1}, 0.42 \mathrm{~cm}^{-1}$ and $0.35 \mathrm{~cm}^{-1}$ corresponds to a surface coverage of $30 \%, 40 \%$ and $130 \%$ (i.e. 1.3 monolayers) for BCP-34 $50 \mathrm{w} \% \mathrm{TiO}_{2}, \mathrm{BCP}-9250 \mathrm{w} \% \mathrm{TiO}_{2}$ and the reference Pilkington sample, respectively. See supplementary information in reference [1] for further details. The integrated area under all three peaks, normalised to the value before irradiation, was used as a quantitative measure for the stearic acid stability on the surface of the ARC. While stearic acid decomposition for samples with $25 \mathrm{wt} \% \mathrm{TiO}_{2}$ loading was rather slow, samples with $37.5 \mathrm{wt} \%$ and $50 \mathrm{wt} \% \mathrm{TiO}_{2}$ showed a rapid decay in FTIR intensity. This is further evidenced in Figure 10.7, where the integrated area of stearic acid absorbance is plotted as a function of irradiation time. The intensity decrease for $37.5 \mathrm{wt} \%$ and $50 \mathrm{wt} \% \mathrm{TiO}_{2}$ loading can be fitted by a zeroth-order reaction rate. Samples derived from BCP-34 exhibited rate constants $k$ of $0.36,3.71$ and $5.10 \times 10^{13}$ molecules/min for $25.0 \mathrm{w} \%, 37.5 \mathrm{wt} \%$ and $50 \mathrm{wt} \%$ $\mathrm{TiO}_{2}$ loading, respectively. Decomposition by ARCs with larger pores due to the assembly by 
BCP-92 could be fitted with rate constants $k$ of $0.36,1.54$ and $3.12 \times 10^{13}$ molecules/min for $25.0 \mathrm{w} \%, 37.5 \mathrm{wt} \%$ and $50 \mathrm{wt} \% \mathrm{TiO}_{2}$ loading, respectively.
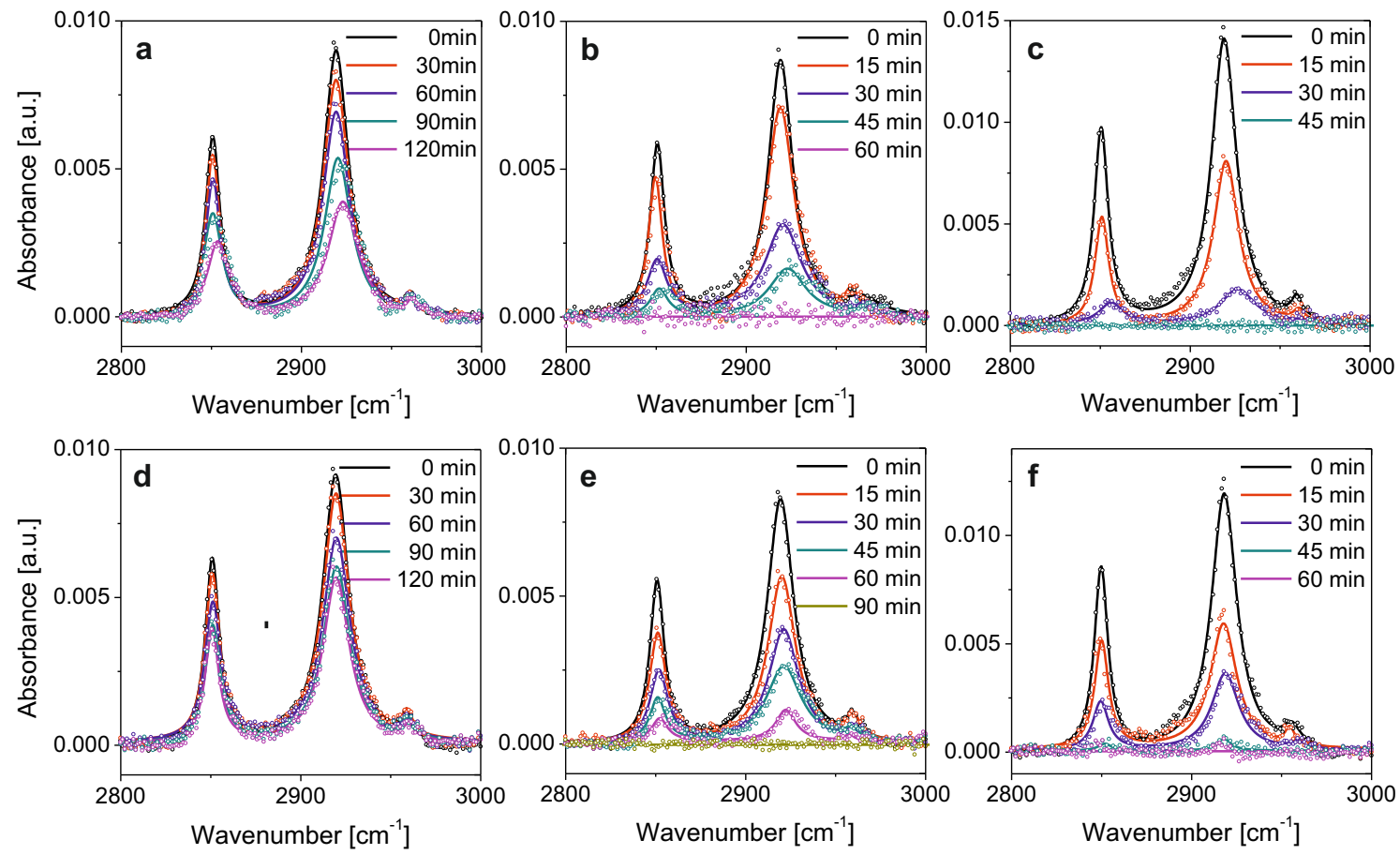

Figure 10.6: Self-cleaning of optical coatings. Decomposition of stearic acid as a function of time for different $\mathrm{TiO}_{2}$ loadings and pore sizes of ARCs. a) - c) ARCs derived from BCP-34 with $\mathrm{TiO}_{2}$ loadings of a) $25 \mathrm{wt} \%$, b) $37.5 \mathrm{wt} \%$ and c) $50 \mathrm{wt} \%$. The decay of the characteristic stearic acid absorption peaks at $\sim 2850 \mathrm{~nm}^{-1}$ and $\sim 2920 \mathrm{~nm}^{-1}$ was monitored by Fourier transform infrared spectroscopy in transmission. d) - e) similar data as in a) - c) for ARCs derived from BCP-92. 

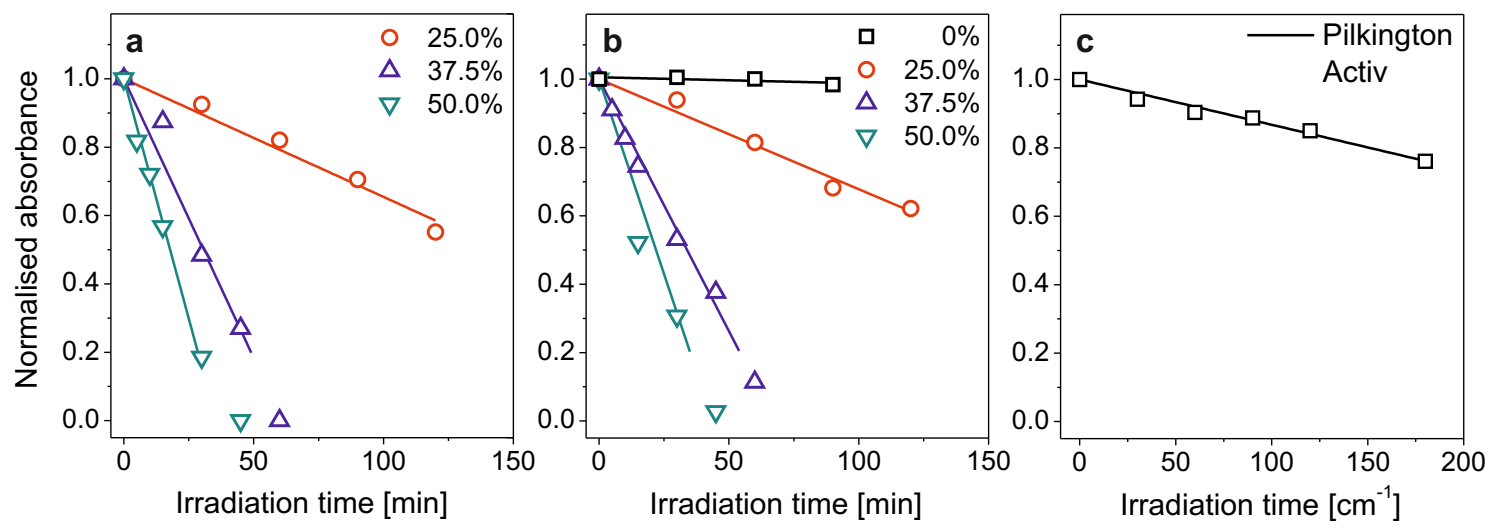

Figure 10.7: Integrated peak area of stearic acid absorbance as a function of irradiation time. The area enclosed by the three characteristic peaks of $\mathrm{CH}_{3}: 2958 \mathrm{~cm}^{-1}, \mathrm{CH}_{2}: 2923 \mathrm{~cm}^{-1}$ and $2853 \mathrm{~cm}^{-1}$, normalised to the initial value before irradiation, was used as a measure of stearic acid stability on the ARCs' surfaces. Peak area vs. time for a) BCP-34 (pore size $\sim 33 \mathrm{~nm}$ ) and b) BCP-92 (pore size $\sim 53 \mathrm{~nm}$ ) derived samples for different $\mathrm{TiO}_{2}$ loadings. c) Decay of stearic acid peak area for a commercial reference (Pilkington Activ) which does not have antireflective properties. 
Two clear trends could be observed. 1. Increased $\mathrm{TiO}_{2}$ loading led to accelerated stearic acid decomposition in both sample series. 2. ARCs with smaller pores (d $33 \mathrm{~nm}$ ) outperformed ARCs with larger pores $(\mathrm{d} \sim 53 \mathrm{~nm})$ for the higher $\mathrm{TiO}_{2}$ content. This behaviour can be linked to the random distribution of $\mathrm{TiO}_{2}$ nanocrystals in the silica-type matrix, shown in Figure 10.5. Both an increase in $\mathrm{TiO}_{2}$ loading and a reduction in strut diameter leads to an increase in the number of nanocrystals that are located close to the strut surface and are therefore photocatalytically active. An overview of the photocatalytic decomposition rates is shown in Table 10.1

For reference, the stearic acid decomposition under solar irradiation was also monitored for ARC samples without $\mathrm{TiO}_{2}$ inclusion. No decrease in FTIR absorbance could be observed after three hours of irradiation, indicating that the stearic acid decomposition solely arises from the photocatalytic activity of the incorporated $\mathrm{TiO}_{2}$ nanocrystals (see supplementary information in reference [1]). Commercial self cleaning Pilkington glass, which is not anti-reflective, showed a much weaker photocatalytic degradation of stearic acid with a reaction rate $k$ of $\sim$ $0.13 \times 10^{13}$ molecules/min. This result is somewhat surprising as the commercial glass is covered by a continuous, non-porous coating of $\mathrm{TiO}_{2}$ that is optimised for self-cleaning without having to achieve a specific refractive index. The greatly decreased photocatalytic activity of Pilkington "Activ" compared to a thin film of $\mathrm{TiO}_{2}$ nanoparticles has already been shown in earlier studies [42]. In addition, the presented route allows the incorporation of purely crystalline $\mathrm{TiO}_{2}$, which is know for its superior photocatalytic activity compared to amorphous $\mathrm{TiO}_{2}$ and mixed crystal phases [44]. While a difference in stearic coverage with $30 \%, 40 \%$ and $130 \%$ (i.e. 1.3 monolayers) was observed for BCP-34, BCP-92 and the reference Pilkington sample, this should not be a decisive factor for the greatly enhanced decomposition in the BCP-derived samples. A zeroth-order decomosition observed in all samples indicates that sufficient "hydrocarbon fuel" was present on the surface throughout the self-cleaning process. A coverage below $100 \%$ rather implies that only a fraction of the ARC's surface is actively decomposing.

Table 10.1: Decomposition rates of self-cleaning samples.

\begin{tabular}{cccc}
\hline Sample name & $\begin{array}{c}\mathrm{TiO}_{2} \text { content } \\
(\mathrm{w} \%)\end{array}$ & slope & $\begin{array}{c}\text { reaction rate } \\
\text { (no. molecules x 10 }\end{array}$ \\
\hline BCP-34 & 50.0 & -0.0280 & 4.71 \\
BCP-34 & 37.5 & -0.0239 & 2.53 \\
BCP-34 & 25.0 & -0.0035 & 0.37 \\
\hline BCP-92 & 50.0 & -0.0228 & 3.32 \\
BCP-92 & 37.5 & -0.0147 & 1.54 \\
BCP-92 & 25.0 & -0.0032 & 0.36 \\
\hline Pilkington reference & n.a. & -0.0013 & 0.15 \\
\hline
\end{tabular}




\subsection{Conclusions}

In this chapter a new concept for the fabrication of inorganic coatings that combine antireflective and photocatalytic properties is presented. The large volume fraction of the BCP's hydrophobic PI block enabled micellisation of the hybrid system under readily achieved nonequilibrium processing conditions. Upon spin coating, the sacrificial colloidal micelles selfassembled in a close-packed lattice. As the inorganic material resided in the hydrophilic outer layer of the micelles, it was co-assembled in an inverse-opal like geometry. Subsequent removal of the polymer yielded structurally stable and continuous inorganic films. Since the porosity arises from the close-packing of pores rather than high refractive index components, porosity values of up to $73 \%$ were achieved. This enabled the substitution of up to $50 \mathrm{wt} \%$ aluminosilicate by high refractive index $\mathrm{TiO}_{2}$ nanocrystals, while maintaining antireflective properties. As a result, the ARCs showed high performance in photocatalytic self-cleaning while exhibiting an excellent optical response. The high abrasion resistance of aluminosilicate films should further allow excellent durability of the coatings [45]. Low temperature processing enabled the deposition onto flexible plastic substrates. It should be straight-forward to extend the film deposition technique to roll-to-roll processing (i.e. slot-die coating or meniscus coating [36]), making this universal route highly attractive for the manufacture of next generation ARCs.

\section{Bibliography}

[1] S. Guldin, P. Kohn, M. Stefik, J. Song, G. Divitini, C. Ducati, U. Wiesner, and U. Steiner, "Self-cleaning antireflective optical coatings," manuscript in preparation.

[2] J. von Fraunhofer, "Versuche über die Ursachen des Anlaufens und Mattwerdens des Glases und die Mittel, densselben zuvorzukommen," Verlag der Königlich Bayerischen Akademie der Wissenschaften, 1817.

[3] L. Raighleigh, "On the intensity of light reflected from certain surfaces at nearly perpendicular incidence," Proceedings of the Royal Society, vol. 41, pp. 275-94, 1886.

[4] H. Macleod, Thin film optical filters. Institute of Physics Publishing, 3rd ed., 2001.

[5] D. Bruggeman, "Calculation of various physics constants in heterogenous substances. I. Dielectricity constants and conductivity of mixed bodies from isotropic substances," Annalen Der Physik, vol. 24, no. 7, pp. 636-664, 1935.

[6] D. Bergman and D. Stroud, "Physical-properties of macroscopically inhomogeneousmedia," in Solid State Physics: Advances In Research And Applications, Vol 46, vol. 46 of Solid State Physics-Advances In Research And Applications, pp. 147-269, Academic Press Inc, 1992. 
[7] N. Hutchinson, T. Coquil, A. Navid, and L. Pilon, "Effective optical properties of highly ordered mesoporous thin films," Thin Solid Films, vol. 518, no. 8, pp. 2141-2146, 2010.

[8] S. Walheim, E. Schäffer, J. Mlynek, and U. Steiner, "Nanophase-separated polymer films as high-performance antireflection coatings," Science, vol. 283, no. 5401, pp. 520-522, 1999.

[9] S. Kim, J. Cho, and K. Char, "Thermally stable antireflective coatings based on nanoporous organosilicate thin films," Langmuir, vol. 23, no. 12, pp. 6737-43, 2007.

[10] H. Hattori, "Anti-reflection surface with particle coating deposited by electrostatic attraction," Advanced Materials, vol. 13, no. 1, pp. 51-54, 2001.

[11] D. Lee, M. Rubner, and R. Cohen, "All-nanoparticle thin-film coatings," Nano Letters, vol. 6, no. 10, pp. 2305-12, 2006.

[12] A. Gombert, W. Glaubitt, K. Rose, J. Dreibholz, B. Bläsi, A. Heinzel, D. Sporn, W. Döll, and V. Wittwer, "Subwavelength-structured antireflective surfaces on glass," Thin Solid Films, vol. 351, no. 1-2, pp. 73-78, 1999.

[13] M. Hawkeye and M. Brett, "Glancing angle deposition: fabrication, properties, and applications of micro- and nanostructured thin films," Journal Of Vacuum Science E Technology A, vol. 25, no. 5, pp. 1317-1335, 2007.

[14] J.-Q. Xi, M. Schubert, J. Kim, E. Schubert, M. Chen, S.-Y. Lin, W. Liu, and J. Smart, "Optical thin-film materials with low refractive index for broadband elimination of fresnel reflection," Nature Photonics, vol. 1, no. 3, pp. 176-179, 2007.

[15] C. Bernhard, "Structural and functional adaptation in a visual system," Endeavour, vol. 26, no. 98, pp. 79-\&, 1967.

[16] S. Wilson and M. Hutley, "The optical-properties of moth eye antireflection surfaces," Optica Acta, vol. 29, no. 7, pp. 993-1009, 1982.

[17] P. Clapham and M. Hutley, "Reduction of lens reflection by moth eye principle," Nature, vol. 244, no. 5414, pp. 281-282, 1973.

[18] Y. Li, J. Zhang, S. Zhu, H. Dong, F. Jia, Z. Wang, Z. Sun, L. Zhang, Y. Li, H. Li, W. Xu, and B. Yang, "Biomimetic surfaces for high-performance optics," Advanced Materials, vol. 21, no. 46, pp. 4731-4734, 2009.

[19] T. Lohmüller, M. Helgert, M. Sundermann, R. Brunner, and J. Spatz, "Biomimetic interfaces for high-performance optics in the deep-uv light range," Nano Letters, vol. 8, no. 5, pp. 1429-1433, 2008.

[20] I. Parkin and R. Palgrave, "Self-cleaning coatings," Journal of Materials Chemistry, vol. 15, pp. 1689-1695, 2005.

[21] X.-T. Zhang, O. Sato, M. Taguchi, Y. Einaga, T. Murakami, and A. Fujishima, "Selfcleaning particle coating with antireflection properties," Chemistry of Materials, vol. 17, no. 3, pp. 696-700, 2005.

[22] X. Zhang, A. Fujishima, M. Jin, A. Emeline, and T. Murakami, "Double-layered $\mathrm{TiO}_{2}$ $\mathrm{SiO}_{2}$ nanostructured films with self-cleaning and antireflective properties.," Journal Of Physical Chemistry B, vol. 110, no. 50, pp. 25142-25148, 2006. 
[23] M. Faustini, L. Nicole, C. Boissiere, P. Innocenzi, C. Sanchez, and D. Grosso, "Hydrophobic, antireflective, self-cleaning, and antifogging sol-gel coatings: an example of multifunctional nanostructured materials for photovoltaic cells," Chemistry Of Materials, vol. 22, no. 15, pp. 4406-4413, 2010.

[24] P. Hartmann, D.-K. Lee, B. Smarsly, and J. Janek, "Mesoporous $\mathrm{TiO}_{2}$ : comparison of classical sol-gel and nanoparticle based photoelectrodes for the water splitting reaction," ACS Nano, vol. 4, no. 6, pp. 3147-3154, 2010.

[25] "Terrestrial reference spectra for photovoltaic performance evaluation, g173-03," American Society for Testing and Materials (ASTM), http://rredc.nrel.gov/solar/spectra/am1.5, 1999.

[26] M. Templin, A. Franck, A. DuChesne, H. Leist, Y. Zhang, R. Ulrich, V. Schädler, and U. Wiesner, "Organically modified aluminosilicate mesostructures from block copolymer phases," Science, vol. 278, no. 5344, pp. 1795-1798, 1997.

[27] M. Niederberger, M. Bartl, and G. Stucky, "Benzyl alcohol and titanium tetrachloride a versatile reaction system for the nonaqueous and low-temperature preparation of crystalline and luminescent titania nanoparticles," Chemistry Of Materials, vol. 14, no. 10, pp. 4364-4370, 2002.

[28] M. Rubinstein and R. Colby, Polymer physics. Oxford University Press, 1st ed., 2003.

[29] H. Jaeger and S. Nagel, "Physics of the granular state," Science, vol. 255, no. 5051, pp. 1523-1531, 1992.

[30] J. Kobler, B. Lotsch, G. Ozin, and T. Bein, "Vapor-sensitive bragg mirrors and optical isotherms from mesoporous nanoparticle suspensions," ACS Nano, vol. 3, no. 7, pp. 1669-1676, 2009.

[31] C. Lopez-Lopez, S. Colodrero, S. Raga, H. Lindstrom, F. Fabregat-Santiago, J. Bisquert, and H. Míguez, "Enhanced diffusion through porous nanoparticle optical multilayers," Journal Of Materials Chemistry, vol. 22, no. 5, pp. 1751-1757, 2012.

[32] L. Landstrom, D. Brodoceanu, N. Arnold, K. Piglmayer, and D. Bäuerle, "Photonic properties of silicon-coated colloidal monolayers," Applied Physics A-Materials Science $\mathcal{E}$ Processing, vol. 81, no. 5, pp. 911-913, 2005.

[33] H.-Y. Hsueh, H.-Y. Chen, M.-S. She, C.-K. Chen, R.-M. Ho, S. Gwo, H. Hasegawa, and E. Thomas, "Inorganic gyroid with exceptionally low refractive index from block copolymer templating," Nano Letters, vol. 10, no. 12, pp. 4994-5000, 2010.

[34] M. Niederberger, "Nonaqueous sol-gel routes to metal oxide nanoparticles," Accounts Of Chemical Research, vol. 40, no. 9, pp. 793-800, 2007.

[35] P. Tanev, M. Chibwe, and T. Pinnavaia, "Titanium-containing mesoporous molecularsieves for catalytic-oxidation of aromatic-compounds," Nature, vol. 368, no. 6469, pp. 321-323, 1994.

[36] F. Krebs, "Fabrication and processing of polymer solar cells: a review of printing and coating techniques," Solar Energy Materials And Solar Cells, vol. 93, no. 4, pp. 394 412, 2009. 
[37] B. G. Kum, Y. C. Park, Y. J. Chang, J. Y. Jeon, and H. M. Jang, "Single-layered porous silica films on polyethylene terephthalate substrates for antireflection coatings," Thin Solid Films, vol. 519, no. 11, pp. 3778-3781, 2011.

[38] T. Yanagishita, K. Nishio, and H. Masuda, "Anti-reflection structures on lenses by nanoimprinting using ordered anodic porous alumina," Applied Physics Express, vol. 2, no. $2,2009$.

[39] K. Nakata, M. Sakai, T. Ochiai, T. Murakami, K. Takagi, and A. Fujishima, "Antireflection and self-cleaning properties of a moth-eye-like surface coated with $\mathrm{TiO}_{2}$ particles," Langmuir, vol. 27, no. 7, pp. 3275-3278, 2011.

[40] P. Kohn, S. Guldin, U. Wiesner, and U. Steiner, "Crystal growth in block copolymer assembled $\mathrm{TiO}_{2}$ networks," in preparation.

[41] Y. Paz, Z. Luo, L. Rabenberg, and A. Heller, "Photooxidative self-cleaning transparent titanium-dioxide films on glass," Journal Of Materials Research, vol. 10, no. 11, pp. 2842-2848, 1995.

[42] A. Mills, A. Lepre, N. Elliott, S. Bhopal, I. Parkin, and S. O'Neill, "Characterisation of the photocatalyst Pilkington Activ: a reference film photocatalyst?," Journal of Photochemistry and Photobiology A: Chemistry, vol. 160, no. 3, pp. 213-224, 2003.

[43] H. Tang, K. Prasad, R. Sanjines, P. Schmid, and F. Levy, "Electrical and optical properties of $\mathrm{TiO}_{2}$ anatase thin-films," Journal Of Applied Physics, vol. 75, no. 4, pp. 2042-2047, 1994.

[44] B. Ohtani, Y. Ogawa, and S. Nishimoto, "Photocatalytic activity of amorphous-anatase mixture of titanium(IV) oxide particles suspended in aqueous solutions," Journal Of Physical Chemistry B, vol. 101, no. 19, pp. 3746-3752, 1997.

[45] H. Schmidt and H. Wolter, "Organically modified ceramics and their applications," Journal Of Non-Crystalline Solids, vol. 121, no. 1-3, pp. 428-435, 1990. 



\section{${ }_{\text {chanerer }} 11$}

\section{Conclusions}

The aim of this thesis was to explore the use of soft matter design principles for improved functionality of inorganic nanoarchitectures. The self-assembly properties of sacrificial block copolymers (BCPs) and colloids were employed as the structure-defining motive for the coassembly of inorganic materials. This allowed detailed control over film thickness, porosity, pore dimensions and accessibility as well as crystal growth of the resulting inorganic material structures, all of which are decisive attributes for the performance of photonic and optoelectronic devices.

Implementation of the proposed materials architectures in dye-sensitised solar cells (DSCs) yielded promising results. $\mathrm{BCP}$-derived $\mathrm{TiO}_{2}$ networks offer superior controllability over pore size distribution and charge transport properties. It proved, however, difficult to outperform the long optimised conventional photoanodes in terms of overall device efficiency. This was recently accomplished with a terblock copolymer PI- $b$-PS- $b$-PEO directed $\mathrm{TiO}_{2}$ photoelectrode, which improved the overall device efficiency of solid-state DSCs compared to the state-ofthe-art nanoparticle system [1]. The fundamental understanding of structure formation in thin films (Chapter 5), crystal growth (Chapter 6) and thick film deposition (Chapter 7) should enable further improvement and hopefully foster the viability of this concept for future devices. Especially with the rise of one-electron-based, bulky redox-couples, such as cobalt-based electrolytes [2], the advantages of BCP-derived photoelectrodes should have a positive impact on further efficiency improvements.

The findings presented in Chapter 6 on superior electronic properties of BCP-assembled photoanodes are certainly only a first step in the right direction. Combining the presented concept with hard scaffolding, i.e. the coating of a sacrificial and temperature stable inorganic layer, could be a viable concept to combine high surface area and outstanding charge carrier transport properties [3]. The use of hierarchical photoanode structures by the combination of 
different structure-directing agents $[4,5]$ as well as surface passivation by using a core-shell strategies $[6,7,8]$ are emerging concepts that are compatible with this work. So far, only thermally-driven crystal growth was explored, but soft matter self-assembly should also be capable to guide the growth of inorganic material by oriented attachment at low temperatures in solution $[9,10,11]$. Other directions for future research directions include the integration of a three-dimensionally structured transparent conductive electrode for radial electron collection, where aspects presented in this work should make further impact [12, 13, 14].

The work on photonic crystal-based optical elements has been well received and a large number of similar studies have recently emerged. In close relation to the work presented in Chapter 9, advances in the fabrication of thick BCP-assembled films (Chapter 7, [15]) and further development in the fabrication of defect free photonic crystals [16, 17] should lead to overall device improvements. The one-dimensional mesoporous Bragg reflectors (MDBRs) presented in Chapter 8 are ideally suited for the use in DSCs and represent a promising platform to further investigate the effect of complementary photonic elements for overall device efficiency improvements. The advantages of pore size and pore dimensions tunability of BCPderived MDBRs have not been yet been fully exploited and should foster next-generation devices with improved functionality for sensing, light-emission and coupled device systems $[18,19]$. Another field that benefits from the complementary use of $1 \mathrm{D}$ and 3D photonic crystals is photocatalytic water splitting [20,21].

In Chapter 5 the possible application of gyroid-like aluminosilica networks as electrodes in lithium ion batteries was discussed. Reduction of the presented aluminosilica films in magnesium vapor is one route to access a continuous silicon network on the $20-40 \mathrm{~nm}$ length scale. Due to the strain associated with the volume change during charging and discharging cycle, networks on the $10 \mathrm{~nm}$ length scale suffer from structural collapse, whereas coarser colloidal templated structures do not exhibit the necessary interfacial area to allow fast lithium insertion $[22,23,24]$. The use of large molecular weight BCPs as structure-directing agents on a somewhat intermediate length scale is therefore an ideal candidate for further device improvement.

The author hopes to have communicated some inspiring possibilities that soft matter selfassembly offers for designing tomorrow's materials. When appropriately utilised, these materials represent a unique toolbox for further development of inorganic materials and devices. Thank you for your interest.

\section{Bibliography}

[1] P. Docampo, M. Stefik, S. Guldin, N. Yufa, R. Gunning, U. Wiesner, U. Steiner, and H. Snaith, "Triblock terpolymer directed self-assembly of mesoporous $\mathrm{TiO}_{2}$ - high per- 
formance photoanodes for solid state dye-sensitized solar cells," Advanced Energy Materials, vol. 2, no. 6, pp. 676-682, 2012.

[2] A. Yella, H.-W. Lee, H. Tsao, C. Yi, A. Chandiran, M. Nazeeruddin, E.-G. Diau, C.Y. Yeh, S. Zakeeruddin, and M. Grätzel, "Porphyrin-sensitized solar cells with cobalt (II/III)-based redox electrolyte exceed 12 percent efficiency," Science, vol. 334, no. 6056, pp. 629-634, 2011.

[3] J. Brillet, M. Grätzel, and K. Sivula, "Decoupling feature size and functionality in solution-processed, porous hematite electrodes for solar water splitting," Nano Letters, vol. 10, no. 10, pp. 4155-4160, 2010.

[4] X. Dang, H. Yi, M.-H. Ham, J. Qi, D. Yun, R. Ladewski, M. Strano, P. Hammond, and A. Belcher, "Virus-templated self-assembled single-walled carbon nanotubes for highly efficient electron collection in photovoltaic devices," Nature Nanotechnology, vol. 6, no. 6, pp. 377-384, 2011.

[5] B. Mandlmeier, J. M. Szeifert, D. Fattakhova-Rohlfing, H. Amenitsch, and T. Bein, "Formation of interpenetrating hierarchical titania structures by confined synthesis in inverse opal," Journal Of The American Chemical Society, vol. 133, no. 43, pp. 17274-17282, 2011.

[6] Y. Diamant, S. Chen, O. Melamed, and A. Zaban, "Core-shell nanoporous electrode for dye sensitized solar cells: the effect of the SrTiO3 shell on the electronic properties of the $\mathrm{TiO}_{2}$ core," Journal Of Physical Chemistry B, vol. 107, no. 9, pp. 1977-1981, 2003.

[7] V. Thavasi, V. Renugopalakrishnan, R. Jose, and S. Ramakrishna, "Controlled electron injection and transport at materials interfaces in dye sensitized solar cells," Materials Science $\mathcal{E}$ Engineering R-Reports, vol. 63, no. 3, pp. 81-99, 2009.

[8] H. Snaith and C. Ducati, " $\mathrm{SnO}_{2}$-based dye-sensitized hybrid solar cells exhibiting near unity absorbed photon-to-electron conversion efficiency," Nano Letters, vol. 10, no. 4, pp. 1259-1265, 2010.

[9] R. Penn and J. Banfield, "Morphology development and crystal growth in nanocrystalline aggregates under hydrothermal conditions: Insights from titania," Geochimica Et Cosmochimica Acta, vol. 63, no. 10, pp. 1549-1557, 1999.

[10] H. Cölfen and S. Mann, "Higher-order organization by mesoscale self-assembly and transformation of hybrid nanostructures," Angewandte Chemie - Int. Ed., vol. 42, no. 21, pp. 2350-2365, 2003.

[11] N. Tetreault, E. Horvath, T. Moehl, J. Brillet, R. Smajda, S. Bungener, N. Cai, P. Wang, S. Zakeeruddin, L. Forro, A. Magrez, and M. Grätzel, "High-efficiency solid-state dyesensitized solar cells: fast charge extraction through self-assembled 3D fibrous network of crystalline $\mathrm{TiO}_{2}$ nanowires," ACS Nano, vol. 4, no. 12, pp. 7644-7650, 2010.

[12] A. Martinson, J. Elam, J. Liu, M. Pellin, T. Marks, and J. Hupp, "Radial electron collection in dye-sensitized solar cells," Nano Letters, vol. 8, no. 9, pp. 2862-2866, 2008.

[13] J. Noh, H. Han, S. Lee, J. Kim, K. Hong, G.-S. Han, H. Shin, and H. Jung, "Nanowirebased three-dimensional transparent conducting oxide electrodes for extremely fast charge collection," Advanced Energy Materials, vol. 1, no. 5, pp. 829-835, 2011. 
[14] N. Tetreault, E. Arsenault, L.-P. Heiniger, N. Soheilnia, J. Brillet, T. Moehl, S. Zakeeruddin, G. Ozin, and M. Grätzel, "High-efficiency dye-sensitized solar cell with threedimensional photoanode," Nano Letters, vol. 11, no. 11, pp. 4579-4584, 2011.

[15] S. Guldin, P. Docampo, M. Stefik, G. Kamita, U. Wiesner, H. Snaith, and U. Steiner, "Layer-by-layer formation of block copolymer derived $\mathrm{TiO}_{2}$ for solid state dye-sensitized solar cells," Small, vol. 8, no. 3, pp. 432-440, 2012.

[16] B. Hatton, L. Mishchenko, S. Davis, K. Sandhage, and J. Aizenberg, "Assembly of large-area, highly ordered, crack-free inverse opal films," Proceedings Of The National Academy Of Sciences Of The United States Of America, vol. 107, no. 23, pp. 1035410359, 2010.

[17] L. Liu, S. Karuturi, L. Su, and A. I. Y. Tok, " $\mathrm{TiO}_{2}$ inverse-opal electrode fabricated by atomic layer deposition for dye-sensitized solar cell applications," Energy E Environmental Science, vol. 4, no. 1, pp. 209-215, 2011.

[18] D. Puzzo, F. Scotognella, M. Zavelani-Rossi, M. Sebastian, A. Lough, I. Manners, G. Lanzani, R. Tubino, and G. Ozin, "Distributed feedback lasing from a composite poly(phenylene vinylene)-nanoparticle one-dimensional photonic crystal.," Nano Letters, vol. 9, no. 12, pp. 4273-8, 2009.

[19] M. Calvo, S. Colodrero, N. Hidalgo, G. Lozano, C. Lopez-Lopez, O. Sanchez-Sobrado, and H. Míguez, "Porous one dimensional photonic crystals: novel multifunctional materials for environmental and energy applications," Energy $\mathcal{E}$ Environmental Science, vol. 4, pp. 4800-4812, 2011.

[20] J. Chen, G. von Freymann, Y. Choi, V. Kitaev, and G. Ozin, "Amplified photochemistry with slow photons," Advanced Materials, vol. 18, no. 14, pp. 1915-1919, 2006.

[21] E. Redel, P. Mirtchev, C. Huai, S. Petrov, and G. Ozin, "Nanoparticle films and photonic crystal multilayers from colloidally stable, size-controllable zinc and iron oxide nanoparticles," ACS Nano, vol. 5, no. 4, pp. 2861-2869, 2011.

[22] A. Esmanski and G. Ozin, "Silicon inverse-opal-based macroporous materials as negative electrodes for lithium ion batteries," Advanced Functional Materials, vol. 19, no. 12, pp. 1999-2010, 2009.

[23] B. Key, R. Bhattacharyya, M. Morcrette, V. Seznec, J.-M. Tarascon, and C. Grey, "Realtime NMR investigations of structural changes in silicon electrodes for lithium-ion batteries," Journal Of The American Chemical Society, vol. 131, no. 26, pp. 9239-9249, 2009.

[24] E. Richman, C. Kang, T. Brezesinski, and S. Tolbert, "Ordered mesoporous silicon through magnesium reduction of polymer templated silica thin films," Nano Letters, vol. 8, no. 9, pp. 3075-3079, 2008. 


\title{
Curriculum vitae of Dr. Stefan Guldin
}

\author{
EDUCATION
}

$10 / 2008-02 / 2012$

University of Cambridge - Ph.D. in physics

Supervisor: Prof. Ullrich Steiner, Cavendish Laboratory

Thesis title: "Inorganic nanoarchitectures by organic self-assembly"

10/2009- 05/2010 University of Cambridge - teaching associate diploma (HEA)

Nine-month programme on teaching and learning in Higher Education

04/2005 - 09/2008 Technical University Munich - diploma in physics

Specialising in semiconductor and soft matter physics

Graduated with distinction (average grade 1.1, summa cum laude)

10/2003 - 04/2005 University of Karlsruhe - prediploma in physics

Theoretical and experimental physics

Prediploma with distinction (average grade 1.1)

09/1992 - 06/2001 Bildungszentrum Markdorf - university qualification

Main subjects: mathematics and politics

Best student in district with an average grade of 1.0 on a 1-6 scale

HONORS AND AWARDS

$12 / 2012$

Postdoctoral Fellowship of the German Academy of Sciences Leopoldina

Early career fellowship for outstanding postdoctoral scientists to develop their own independent research.

11/2012 Springer Outstanding Thesis Award

Publication award by Springer for outstanding $\mathrm{PhD}$ research. 
$11 / 2010$

$06 / 2009$

$06 / 2007$

$04 / 2004-09 / 2008$

$03 / 1999$

\section{NanoDTC Asssociate Student Grant}

Travel grant for outstanding doctoral candidates to promote crossdisciplinary research within the center themes of self-assembling functional nano-materials and devices.

\section{EMRS Young Scientist Award}

In recognition of the outstanding paper contributed to the 2009 spring meeting of the European Materials Research Society.

\section{Hölderlin Scholarship}

Stipend for a research year abroad funded by the Studienstiftung des deutschen Volkes and Siemens.

\section{Fellow of the "Studienstiftung des deutschen Volkes"}

Program of the German government to support highly talented and socially committed students (top $0.5 \%$, enabling fully funded undergraduate studies).

\section{Markdorfer Wirtschaftspreis}

Scholarship for a 3 month stay at Columbus East High School, Indiana/USA (regional award for highly talented students).

\section{WORK EXPERIENCE}

since $09 / 2012$

$08 / 2007-08 / 2012$

06/2009 - 08/2009

03/2007-04/2007
École Polytechnique Fédérale de Lausanne, Lausanne, $\mathrm{CH}$

Experimental research in the field of nanoparticles and soft matter self-assembly.

\section{University of Cambridge, Cambridge, UK}

Experimental research in the field of soft matter physics and functional materials. See below for details on acquired scientific skills.

\section{Cornell University, Ithaca, NY, USA}

Scientific stay in the group of Prof. Ulrich Wiesner. Work focus on materials chemistry, electron microscopy and small angle x-ray scattering.

Science resort of the Bavarian Broadcasting Corp., Munich, GER Independent production of a 4 min documentary about energy saving lamps in the program NANO (200,000 viewers). 
01/2006 - 06/2007 Bosch and Siemens Home Appliances, Munich, GER

Part time position initially on the thermoacoustics of a plant oil cooker. In 2007 development of a financial concept under the Kyoto clean development mechanism to stimulate the distribution of a plant oil cooker in developing countries.

10/2004 - 02/2005 Industrial Research Itd., Christchurch, NZ

Internship on the development of a heat exchanger for a fuel cell device.

08/2001 - 05/2002 Civilian service Kiel, GER

Care and nursing of disabled adults.

since $07 / 1997$

Boarder's point by Sport Guldin, Markdorf, GER

Management of the self-founded snowboard department "Boarder's point" in the sport store owned by my parents. Organization of noncommercial ski- and snowboard excursions for young people with currently 4,000 participants per year (since 2001 in consulting function).

\section{TEACHING}

$2009-2011$

Supervisor "Introduction to Experimental and Theoretical Physics", $1^{\text {st }}$ year physics, Physics Department, University of Cambridge, UK

Supervisor "Soft matter \& Biophysics", $4^{\text {th }}$ year physics, Physics Department, University of Cambridge, UK

Research co-supervisor "Plasmonic Enhancement of DyeSensitized Solar Cells", physics $4^{\text {th }}$ year project, Tjonnie G.F. Li, University of Cambridge, UK

Lab demonstrator "Systems \& Measurements", 2 $2^{\text {nd }}$ year physics, Physics Department, University of Cambridge, UK 


\section{SCIENTIFIC SKILLS}

Material Fabrication Atomic layer deposition ${ }^{\mathrm{B}}$, block copolymer templating ${ }^{\mathrm{A}}$, colloidal templating ${ }^{\mathrm{A}}$, chemical vapor deposition ${ }^{\mathrm{B}}$, reactive ion etching ${ }^{\mathrm{A}}$, solgel chemistry ${ }^{\mathrm{A}}$, solution processing ${ }^{\mathrm{A}}$

Microscopy Confocal microscopy ${ }^{\mathrm{B}}$, scanning electron microscopy ${ }^{\mathrm{A}}$, transmission electron microscopy ${ }^{\mathrm{B}}$

Scattering Dynamic light scattering ${ }^{\mathrm{A}}$, small-angle $\mathrm{X}$-ray scattering ${ }^{\mathrm{B}}$, wide-angle X-ray scattering ${ }^{\mathrm{A}}$

Other Techniques Atomic force microscopy ${ }^{\mathrm{B}}$, ellipsometry ${ }^{\mathrm{A}}$, micro-spectroscopy ${ }^{\mathrm{A}}$, nitrogen physisorption ${ }^{\mathrm{B}}$, photovoltaic device characterization ${ }^{\mathrm{A}}$, thermogravimetric analysis $^{\mathrm{B}}$, uv-vis spectroscopy ${ }^{\mathrm{B}}$, FT-IR spectroscopy ${ }^{\mathrm{B}}$

Computing Adobe Photoshop ${ }^{\mathrm{A}}$, CorelDraw ${ }^{\mathrm{A}}$, LaTeX ${ }^{\mathrm{A}}$, Matlab ${ }^{\mathrm{B}}$, Microsoft Office $^{\mathrm{A}}$, Origin ${ }^{\mathrm{A}}$

Languages German (mother language), English (fluent), French ${ }^{\mathrm{A}}, \mathrm{Spanish}^{\mathrm{A}}$

A advanced, ${ }^{\mathrm{B}}$ basic

\section{OTHER SCIENTIFIC ACTIVITIES}

Organiser of Main conference organiser of the "Soft Nanophotonics Winterschool" in 2009, 2010 and 2011 (University of Cambridge, 40-60 participants per year)

Reviewer for

ACS Applied Materials \& Interfaces, ChemSusChem, Electrochemistry Communications, Energy \& Environmental Science, Journal of the American Chemical Society, Journal of Physics D: Applied Physics, Langmuir, Nanotechnology

\section{PATENTS}

Pending

Self-cleaning antireflective optical coatings - S. Guldin, U. Steiner, UK patent application 1212361.8, 11/07/2012. 
PUBLICATIONS (ResearcherID A-7209-2011)

[19] Ordered mesoporous titania from highly amphiphilic block copolymers: tuned solution conditions enable morphology control - M. Stefik, J. Song, H. Sai, M.C. Orilall, S. Guldin, P. Boldrighini, U. Steiner, Sol M. Gruner, U. Wiesner, in preparation.

[18] Novel Fabrication Method of Gyroid and Worm-like ZnO Mesoporous Structures for Photovoltaic Applications - E. Kim, Y. Vaynzof, S. Guldin, U. Steiner, in preparation.

[17] Effect of DIO solvent additive on the stability of PTB7:PCBM solar cells - P.E. Hopkinson, B. Schmidt-Hansberg, S. Guldin, N. Greenham, in preparation.

[16] Crystal nucleation and growth in mesoporous $\mathrm{TiO}_{2}$ - P. Kohn, S. Guldin, U. Wiesner, U. Steiner, in preparation.

[15] Transport limitations in self-assembled photoanodes for solid-state dye-sensitized solar cells - P. Docampo, S. Guldin, U. Steiner, H.J. Snaith, under review.

[14] Self-cleaning antireflective optical coatings - S. Guldin, P. Kohn, M. Stefik, J. Song, G. Divitini, C. Ducati, U. Wiesner, U. Steiner, under formal review at Nature Communications.

[13] Pore-filling of spiro-OMeTAD in solid-state DSCs determined via optical reflectometry - P. Docampo, A. Hey, S. Guldin, R. Gunning, U. Steiner, H.J. Snaith, Advanced Functional Materials, vol. 22, pp. 5010-5019, 2012.

[12] Biomimetic Layer-by-Layer Assembly of Artificial Nacre - A. Finnemore, P.S. Cunha, T. Shean, S. Vignolini, S. Guldin, M. Oyen, U. Steiner, Nature Communications, vol. 3, art no. 966, DOI: 10.1038/ ncomms1970, 2012.

[11] Triblock terpolymer directed self-assembly of mesoporous $\mathrm{TiO}_{2}$ - high performance photoanodes for solid state dye-sensitised solar cells - P. Docampo, M. Stefik, S. Guldin, N.A. Yufa, R. Gunning, U. Wiesner, U. Steiner, H.J. Snaith, Advanced Energy Materials, vol. 2, pp. 676-682, 2012.

[10] A 3D optical metamaterial made by chiral self-assembly - S. Vignolini, N.A. Yufa, P.S. Cunha, S. Guldin, I. Rushkin, M. Stefik, K. Hur, U. Wiesner, J.J. Baumberg, U. Steiner, Advanced Materials, vol. 24, pp. OP23-OP27, 2012.

[09] Layer-by-layer formation of block copolymer derived $\mathrm{TiO}_{2}$ for solid state dyesensitised solar cells - S. Guldin, P. Docampo, M. Stefik, G. Kamita, U. Wiesner, H.J. Snaith, U. Steiner, Small, vol. 8, pp. 432-440, 2012. 
[08] Mesoporous Bragg reflectors - block-copolymer self-assembly leads to building blocks with well defined continuous pores and high control over optical properties, S. Guldin, M. Kolle, M. Stefik, U. Wiesner, U. Steiner, Proceedings of the SPIE, vol. 8095, DOI: 10.1117/12.893818, 2011.

[07] Self-assembly as a design tool for the integration of photonic structures into excitonic solar cells, S. Guldin, P. Docampo, S. Hüttner, P.Kohn, M. Stefik, H.J. Snaith, U. Wiesner, U. Steiner, Proceedings of the SPIE, vol. 8111, DOI: 10.1117/12.893798, 2011.

[06] Tunable mesoporous $\mathrm{TiO}_{2}$ Bragg reflectors based on block-copolymer self-assembly - S. Guldin, M. Kolle, M. Stefik, R. Langford, U. Wiesner, U. Steiner, Advanced Materials, vol. 23, no. 32, pp. 3664-3668, 2011.

[05] Improved conductivity in dye-sensitized solar cells through block-copolymer confined $\mathrm{TiO}_{2}$ crystallization - S. Guldin, S. Hüttner, P. Tiwana, M.C. Orilall, B. Ülgüt, M. Stefik, P. Docampo, M. Kolle, G. Divitini, C. Ducati, S.A.T. Redfern, H.J. Snaith, U. Wiesner, D. Eder, U. Steiner, Energy and Environmental Science, vol. 4, no. 1, pp. 225-233, 2011.

[04] Dye-sensitized solar cell based on a 3D photonic crystal - S. Guldin, S. Hüttner, M. Kolle, M. Welland, P. Müller-Buschbaum, R. Friend, U. Steiner, N. Tetreault, Nano Letters, vol. 10, no. 7, pp. 2303-2309, 2010.

[03] Control of solid-state dye-sensitized solar cell performance by block copolymer directed $\mathrm{TiO}_{2}$ synthesis - P. Docampo*, S. Guldin*, M. Stefik, P. Tiwana, M.C. Orilall, S. Hüttner, H. Sai, U. Wiesner, U. Steiner, H.J. Snaith, Advanced Functional Materials, vol. 11 , no. 20, pp. 1787-1796, 2010.

[02] Monolithic route to efficient dye-sensitized solar cells employing diblock copolymers for mesoporous $\mathrm{TiO}_{2}$ - M. Nedelcu*, S. Guldin*, M.C. Orilall, J. Lee, S. Hüttner, E.J.W. Crossland, S.C. Warren, C. Ducati, P.R. Laity, D. Eder, U. Wiesner, U. Steiner, H.J. Snaith, Journal of Materials Chemistry, vol. 7, no. 20, pp. 1261-1268, 2010.

[01] Block-copolymer directed synthesis of mesoporous $\mathrm{TiO}_{2}$ for dye-sensitized solar cells - M. Nedelcu, J. Lee, E.J.W. Crossland, S.C. Warren, M.C. Orilall, S. Guldin, S. Hüttner, C. Ducati, D. Eder, U. Wiesner, U. Steiner, H.J. Snaith, Soft Matter, vol. 1, no. 5, pp. 134-139, 2009.

* authors contributed equally to this work. 


\section{TALKS AND CONFERENCE PARTICIPATION}

27 oral and poster contributions in seminars, workshops and conferences, including:

[01] Mesoporous Bragg reflectors - block-copolymer self-assembly leads to building blocks with well defined continuous pores and high control over optical properties, talk, SPIE Optics \& Photonics, August 2011, San Diego, USA.

[02] Tunable mesoporous Bragg reflectors based on block-copolymer self-assembly, conference poster prize, 5th International Conference on Nanophotonics, May 2011, Shanghai, China.

[03] Photonic sensing with tunable mesoporous Bragg reflectors based on block-copolymer self-assembly, talk in invited session on bioinspired Nanophotonics, Conference of the European Materials Research Society, May 2011, Nice, France.

[04] Self-assembly as a design tool for the integration of photonic structures into excitonic solar cells, invited talk, Symposium on Nanophotonics and Renewable Energy, Chinese Academy of Sciences, January 2011, Beijing, China.

[05] Catching the light - self-assembled $\mathrm{TiO}_{2}$ nanostructures in a double layer dyesensitized solar cell, conference poster prize, Conference on self-assembly and selforganization at surfaces and interfaces, EU Patterns network, December 2008, Selwyn College, Cambridge, UK.

\section{REFEREES}

[01] Prof. Ulrich Steiner (doctoral supervisor)

John Humphrey Plummer Professor of Physics of Materials

Dept. of Physics, Cavendish Laboratory, Cambridge, CB3 OHE, UK

Telephone: +44 (0) 1223337390

E-mail: u.steiner@phy.cam.ac.uk

[02] Prof. Ullrich Wiesner (collaborator)

Spencer T. Olin Professor of Engineering

Dept. of Materials Science, Cornell University, Ithaca, NY 14853, USA

Telephone: +16072553487

E-mail: ubw1@cornell.edu

[03] Prof. Sir Richard Friend (examiner of Ph.D. thesis)

Cavendish Professor 
Dept. of Physics, Cavendish Laboratory, Cambridge, CB3 0HE, UK

Telephone: +44 (0) 1223337218

E-mail: rhf10@cam.ac.uk

[04] Prof. Thomas Bein (examiner of Ph.D. thesis)

Chair for Physical Chemistry II

Dept. of Chemistry, University of Munich (LMU), 81377 München, GER

Telephone: +49 (0)89218077623

E-mail: bein@lmu.de

[05] Prof. Francesco Stellacci (scientific advisor since 09/2012)

Constellium Professor

Inst. of Materials, École Polytechnique Fédérale de Lausanne, 1015 Lausanne, CH

Telephone: +41 (0)21 6937872

E-mail: Francesco.Stellacci@epfl.ch 